Universidade de São Paulo

Instituto de Física

\title{
Flutuações do Campo Eletromagnético no Regime de Transparência Eletromagneticamente Induzida
}

\author{
Felippe Alexandre Silva Barbosa \\ Orientador: Prof. Dr. Paulo Alberto Nussenzveig \\ Dissertação de mestrado apresentada ao \\ Instituto de Física para a obtenção \\ do título de Mestre em Ciências
}

Banca Examinadora:

Prof. Dr. Flávio Caldas da Cruz (UNICAMP)

Prof. Dr. Emerson José Veloso de Passos (IF-USP)

Prof. Dr. Paulo Alberto Nussenzveig (IF-USP)

São Paulo 


\section{Agradecimentos}

Agradeço primeiramente ao meu orientador, Prof. Paulo Alberto Nussenzveig, pela orientação, pelas discussões sobre as sutilezas do trabalho e pelo apoio demonstrado durante o mestrado, principalmente na escrita da dissertação.

Ao Prof. Marcelo Martinelli pela sua grande ajuda ao solucionar os problemas no laboratório. Pude aprender bastante com a sua experiência como físico experimental.

Ao Paulo Valente pelo apoio, orientação e ajuda dado durante a realização deste trabalho, tanto no entendimento da teoria quanto no domínio do aparato experimental. Sem sua presença boa parte do trabalho desenvolvido não seria possível.

Aos meus pais, Maria Ester e Simplício Barbosa, aos meus irmãos, Túlio e Ícaro, e à minha madrinha, Maria, pelo amor, apoio, educação e valores que me foram dados durante a minha vida. Em especial, o apoio dado durante o curso, não somente deste trabalho, mas de toda a minha formação acadêmica.

Aos meus amigos e familiares cujos nomes, para não correr o risco de esquecer algum, não citarei, agradeço por terem moldado, alguns mais outros menos, minha personalidade e valores como são.

Aos amigos e companheiros de trabalho: o Rodrigo, que me ajudou bastante na aquisição dos dados apresentados, o Antônio, o Laércio, o Fábio e o Jonatas pelas horas descontraídas que ajudaram a amenizar o clima, muitas vezes tenso, do laboratório e pelas discussões sobre física que muito contribuíram para o meu aprendizado.

Aos "juvenais" que moram comigo, Jonatas e João. Agradeço pelas festinhas na USP, e fora dela também, além dos bons momentos divididos e por tornar a vida nesta cidade bem mais divertida.

À Fran e ao Eber da CPG, às secretárias da FEP, Edi e Ivanei, por terem facilitado a minha vida tantas vezes e me mostrado que nem tudo no serviço público é burocracia.

Um agradecimento especial deve ser dado ao meu primeiro mentor na graduação, Prof. Ademir Eugênio de Santana, por quem tenho grande admiração. Pude aprender muito 
com ele em meu tempo de iniciação científica, época fundamental para que eu recuperasse o gosto pela Física.

E finalmente, mas não menos importante, ao CNPq pelo fomento. 
Dedicado à memória de minha avó Isabel. 
"We are all apprentices in a craft where no one ever becomes a master"

Ernest Hemingway 


\section{Resumo}

A Transparência Eletromagneticamente Induzida tem sido muito usada atualmente para implementação de memórias quânticas. Neste trabalho, estudamos este fenômeno, experimental e teoricamente, utilizando um sistema de dois níveis com degenerescências como modelo para o átomo. Tratamos o campo eletromagnético como quantizado e propomos um modelo linearizado para as flutuações que leva em consideração as forças quânticas de Langevin. Devido ao excesso de ruído de fase presente em nosso laser, a implementeção numérica do modelo levou em consideração apenas a autocorrelação de fase como fonte para o sinal de ruído observado. Este modelo mostrou um bom acordo qualitativo com os dados experimentais observados.

Fizemos medidas de ruído de soma e subtração com o esquema de deteção balanceada, calculamos os coeficientes de correlação normalizado e mostramos que há regiões de correlação e anticorrelação que variam com a intensidade do feixe. Observamos também uma inversão de picos no sinal de soma não prevista pelo modelo à medida que diminuimos a intensidade. Esta inversão pode ser causada por contribuições das flutuações de amplitude do laser a baixas intensidades. 


\section{Abstract}

Electromagnetically Induced Transparency has attracted much attention recently as a source to implement quantum memories. In this work, we studied this phenomenon, both theoretically and experimentally, using a degenerate two-level system modeling the atoms. We quantize the electromagnetic field and propose a linearized model for fluctuations that takes into account the quantum Langevin forces. Because of the excess noise in the phase of our laser, the numerical implementation of the theoretical model takes into account only the phase autocorrelation term as a source for the observed noise. This model showed a good qualitative agreement with the experimental data.

We made sum and subtraction noise measurements using the balanced detection scheme, we calculated the normalized correlation coefficient and we showed that there are regions of correlation and anticorrelation that change with laser intensity. We also observed a peak inversion of the sum signal as laser intensity becames smaller that was not predicted by the model with only phase noise. The cause for this inversion can be the contribution of laser amplitude noise at small intensities. 


\section{Sumário}

1 Introdução $\quad$ p. 11

2 Conceitos Introdutórios de Ótica e Mecânica Quântica p. 13

2.1 Conceitos Preliminares . . . . . . . . . . . . . . . p. 13

2.2 Operador Densidade . . . . . . . . . . . . . . . . p. 15

2.3 Relações de Incerteza e Flutuações Quânticas ～. . . . . . . . . . . . p. 17

2.4 Teorema de Wigner-Eckart para Operadores Vetoriais . . . . . . . . p. 19

2.5 Decomposição em Modos Normais . . . . . . . . . . . . . . . . . p. 21

2.6 Quantização do Campo Eletromagnético Livre . . . . . . . . . . . p. 25

2.7 Estados Coerentes . . . . . . . . . . . . . . . . p. 27

2.8 Operadores no Domínio da Frequência . . . . . . . . . . . . . . . p. 29

2.9 Quadraturas do Campo Eletromagnético . . . . . . . . . . . . p. 30

2.10 Funções de Correlação . . . . . . . . . . . . . . . . . . . . p. 33

3 Sistema de 2 Níveis $\quad$ p. 36

3.1 Formulação do Problema . . . . . . . . . . . . . . . . . p. 36

3.2 Equações de Heisenberg-Langevin . . . . . . . . . . . . . . . . . . . . . p. 39

3.3 Equações Óticas de Bloch . . . . . . . . . . . . . . . . . p. 46

3.4 Operador Polarização . . . . . . . . . . . . . . . . p. . . . . .

3.5 Equações para as Flutuações Atômicas e Espectro de Ruído . . . . . . p.53

4 EIT - Sistema de 3 Níveis $\quad$ p. 60

4.1 Formulação do Problema . . . . . . . . . . . . . . . . . p p 60 
4.2 Campos em Ressonância - Estado Escuro . . . . . . . . . . . . . p. 61

4.3 Equações de Bloch . . . . . . . . . . . . . . . . . . . . p. 66

5 Sistema de 2 Níveis com Degenerescências $\quad$ p. 70

5.1 Hamiltoniano da Interação Átomo-Luz . . . . . . . . . . . . . . . . . . p.71

5.2 Efeito Hanle e Interação com o Campo Magnético ～. . . . . . . . . . . p.72

5.3 Equações de Heisenberg-Langevin . . . . . . . . . . . . . . . . . . p.73

5.4 Contribuições Dissipativas . . . . . . . . . . . . . . . . . . p. 74

5.5 Contribuição do Campo Incidente . . . . . . . . . . . . . . . . . . p. 78

5.6 Equações de Bloch no Espaço de Liouville . . . . . . . . . . . . . p. 80

5.7 Equação para as Flutuações e Espectro de Ruído . . . . . . . . . . . . p. 84

6 Métodos Experimentais $\quad$ p. 88

6.1 Átomo de Rubídio . . . . . . . . . . . . . . . . p. 88

6.2 Alargamento Doppler . . . . . . . . . . . . . . . . . . . . . . p. 89

6.3 Absorção Saturada . . . . . . . . . . . . . . . . . . p. p1

6.4 Laser de Diodo . . . . . . . . . . . . . . . . . . . p. 94

6.5 Moduladores Acusto-Óticos em Dupla Passagem . . . . . . . . . . . p. 96

6.6 Fotodiodos . . . . . . . . . . . . . . . . p. 98

6.7 Deteção Homodina Balanceada . . . . . . . . . . . . . . . . p. 98

6.8 Analisador de Espectro . . . . . . . . . . . . . . . . p. 103

6.9 Sistema de Travamento da Frequência dos Lasers . . . . . . . . . . . p. 105

7 Descrição do Aparato Experimental e Resultados Obtidos p.106

7.1 Aparato Experimental . . . . . . . . . . . . . p. 106

7.1.1 Campo Magnético e Célula de Vapor . . . . . . . . . . . p. 107

7.2 Medidas do Shot Noise e Ruído de Subtração . . . . . . . . . . . . p. 108

7.3 Ruído de Soma e Subtração e Coeficiente de Correlação . . . . . . . . p. p. 109 
7.4 Medidas com Lâminas de Meia-Onda . . . . . . . . . . . . . . . p. 111

7.5 Medidas com Lâminas de Quarto-de-Onda . . . . . . . . . . . . . . . . p. 114

8 Conclusões e Perspectivas p. 119

Apêndice A - Hamiltoniano de Dipolo p. 121

$\begin{array}{lr}\text { Apêndice B - Espaço de Liouville } & \text { p. } 123\end{array}$

Apêndice C - Espectro de Ruído e Teorema de Wiener-Khinthchine p.127

$\begin{array}{lr}\text { Referências Bibliográficas } & \text { p. } 129\end{array}$ 


\section{Introdução}

O fenômeno da Transparência Eletromagneticamente Induzida (no inglês EIT) [1] consiste na dimunição da absorção da luz por um meio atômico quando dois campos estão ressonantes com duas transições distintas, tendo um estado em comum. Este fenômeno é explicado por meio de um processo de interferência destrutiva entre canais de excitação. O sistema mais simples em que a EIT pode ocorrer é na configuração de três níveis em $\Lambda$, em que a configuração de níveis atômica é formada por um estado excitado e dois fundamentais. Este tipo de sistema também permite a ocorrência de outros fenômenos, além da EIT, que involvem coerências entre os estados fundamentais, como amplificação sem inversão de população e mistura de quatro ondas. Esta classe de fenômenos é chamada de Aprisionamento Coerente de População (CPT) e é descrita em [2].

O primeiro fenômeno de CPT foi demonstrado em [3], cujo experimento consistia em observar a fluorescência emitida por átomos de sódio presentes em uma região com campo magnético espacialmente variável à medida que interagiam com um feixe de prova próximo à ressonância. Foram vistas franjas claras e escuras no sinal de fluorescência medido na direção perpendicular à do feixe. Este fenômeno foi chamado de ressonância escura e foi explicado pela bombeamento ótico para uma superposição de estados fundamentais que não se acopla com a luz, denominado estado escuro. A primeira observação da EIT em um sistema de três níveis em configuração $\Lambda$ ocorreu em [4], onde o nome EIT foi primeiramente utilizado. Neste experimento, foi medida a intensidade da luz transmitida e esta apresentava uma queda à medida que os dois campos entravam em ressonância.

A EIT é o cerne de uma das propostas para a implementação de memórias quânticas [5]. Esta se resume em armazemar e recuperar o estado quântico da luz incidente nos átomos. As informações presentes na luz são guardadas nas coerências atômicas entre os estados fundamentais criadas na EIT. As mesmas são robustas o suficiente para guardar um possível emaranhamento presente no feixe incidente por um certo período de tempo. O emaranhamento é uma das propriedades mais interessantes apresentadas por sistemas quânticos, pois não é obtida em sistemas clássicos e é de fundamental importância para 
aplicações em informação quântica [6]. Diversas implementações de memórias quânticas por meio de EIT foram feitas nos últimos anos, tanto no regime contínuo utilizando células de vapor de césio [7] e rubídio [8] ou átomos frios [9], quanto discreto com células de vapor $[10,11]$ e átomos frios [12].

Vários aspectos da EIT foram estudados ao longo da última década. Em particular, as flutuações de fase e intensidade para um átomo de três níveis foram estudadas teoricamente em casos dentro e fora de uma cavidade ótica $[13,14,15,16,17]$ e experimentalmente $[18,19]$. As flutuações no regime de EIT em um sistema de dois níveis com degerescências também foram estudadas em nosso laboratório e no grupo do Prof. Arturo Lezama, sendo tema de artigos teóricos e experimentais [20, 21] e teses [22, 23, 24, 25]. O presente trabalho segue uma linha natural traçada pelos anteriores tanto nos quesitos teóricos quanto experimentais.

No segundo capítulo, é apresentada uma revisão sobre conceitos de Mecânica Quântica e Ótica Quântica bem conhecidos e que serão importantes no desenvolvimento da dissertação. No terceiro capítulo estudaremos o átomo de dois níveis interagindo com o campo eletromagnético quantizado. Calcularemos as relações para valores médios e para as flutuações nas variáveis do campo após a interação. O tema do quarto capítulo é a interação do campo com um átomo de três níveis em configuração $\Lambda$. Mostraremos a existência do estado escuro neste tipo de sistema e que a EIT pode ser vista como um fenômeno de interferência destrutiva entre canais de excitação. No quinto capítulo trataremos do sistema de dois níveis com degenerescências. Faremos uma generalização da análise apresentada no terceiro capítulo, tratando separadamente a dinâmica dos valores médios e das flutuações. Também será calculado o espectro de ruído supondo que o feixe laser que interage com os átomos possua grande excesso de ruído de fase. O sexto e sétimo capítulos serão sobre a parte experimental do trabalho. No sexto capítulo, faremos uma descrição detalhada de todos os métodos e medidas de controle necessários para realizar o experimento. No sétimo capítulo serão apresentados os resultados experimentais obtidos e uma discussão sobre os aspectos que confirmam os resultados anteriores e novos resultados que foram observados: a passagem de regiões de correlação para regiões de anticorrelação em função da intensidade e uma inversão de picos no sinal de soma para baixas intensidades. 


\section{Conceitos Introdutórios de Ótica e Mecânica Quântica}

Neste capítulo introduiziremos conceitos preliminares de Mecânica Quântica e Ótica Quântica que serão utilizados no decorrer deste trabalho tanto no desenvolvimento da teoria quanto em alguns aspectos do controle do experimento e do processo de medição. As idéias descritas aqui são apresentadas com o objetivo principal de fixar a notação e são encontradas em diversos livros texto de Mecânica Quântica [26, 27, 28, 29].

\subsection{Conceitos Preliminares}

Na Mecânica Quântica estamos interessados na evolução temporal para o estado de algum sistema físico, assim como em quase todas as teorias físicas. O estado físico, como na Mecânica Clássica, representa toda a informação que podemos obter sobre o sistema. Esta evolução temporal é descrita pela equação de Schrödinger,

$$
i \hbar \frac{\partial}{\partial t}|\psi(t)\rangle=H(t)|\psi(t)\rangle
$$

ou, de forma equivalente, pelo operador de evolução temporal,

$$
\begin{aligned}
|\psi(t)\rangle & =U\left(t, t_{0}\right)\left|\psi\left(t_{0}\right)\right\rangle, \\
U\left(t, t_{0}\right) & =\exp \left[-\frac{i}{\hbar} \int_{t_{0}}^{t} H\left(t^{\prime}\right) d t^{\prime}\right]
\end{aligned}
$$

sendo a última expressão válida somente quando $\left[H(t), H\left(t^{\prime}\right)\right]=0$. O vetor de estado pode ser expandido em uma base ortonormal, $|n\rangle$, tal que

$$
|\psi(t)\rangle=\sum_{n} \psi_{n}(t)|n\rangle
$$


onde $\psi_{n}(t)=\langle n \mid \psi(t)\rangle$ e vale a relação de fechamento para os projetores,

$$
\sum_{n} P_{n}=1
$$

definidos como $P_{n}=|n\rangle\langle n|$.

Como qualquer equação diferencial com condições de contorno determinadas, a equação de Schrödinger possui solução única. Porém, diferentemente da Mecânica Clássica, onde o estado do sistema pode ser medido, o estado na Mecânica Quântica não é diretamente medido, mas usado para calcular a média de observáveis, definida como

$$
\langle\hat{O}\rangle=\langle\psi(t)|\hat{O}| \psi(t)\rangle
$$

Os observáveis são operadores hermitianos que descrevem grandezas físicas e os valores possíveis de serem medidos para os mesmos são os seus autovalores,

$$
\hat{O}|n\rangle=o_{n}|n\rangle
$$

Usando estas duas expressões, junto com (2.4), podemos mostrar que a probabilidade de que se obtenha determinada medida é

$$
p_{n}(t)=|\langle n \mid \psi(t)\rangle|^{2}
$$

Neste sentido, dizemos que a Mecânica Quântica é intrinsecamente probabilísica, pois mesmo a formulação mais fundamental da teoria é feita em bases probabilísticas.

Já que o vetor de estado não é o objeto a ser medido, a sua evolução temporal acaba se tornando arbitrária desde que não afete os parâmetros que podem ser medidos, ou seja, as relações (2.5), (2.6) e (2.7). Podemos escrever (2.5) tal que

$$
\langle\hat{O}\rangle=\left\langle\psi(0)\left|U^{\dagger}(t) \hat{O} U(t)\right| \psi(0)\right\rangle
$$

onde $U^{\dagger}(t) \equiv U^{\dagger}(t, 0)$. Sendo assim, em vez de realizar a evolução temporal no vetor de estado por meio de (2.1) ou (2.2), podemos fazê-la no operador, $\hat{O}(t)=U^{\dagger}(t) \hat{O} U(t)$. Diferenciando esta expressão obtemos a equação de Heisenberg,

$$
i \hbar \frac{d \hat{O}(t)}{d t}=[\hat{O}(t), H]
$$

que é válida somente para $H$ independente do tempo. Quando a evolução temporal é feita sobre o vetor de estado dizemos que estamos no formalismo de Schrödinger e quando esta é relizada sobre os operadores dizemos que estamos no formalismo de Heisenberg. Para 
garantir que em (2.6) o autovalor não mude ao realizar a evolução no observável, $|n\rangle$ deve evoluir no tempo como

$$
|n ; t\rangle=U^{\dagger}(t)|n\rangle
$$

tal que $\hat{O}(t)|n ; t\rangle=n|n ; t\rangle$. Como esperado, esta última condição também garante que a probabilidade de se medir determinado autovalor não seja afetada, pois $\langle n \mid \psi(t)\rangle=$ $\langle n ; t \mid \psi(0)\rangle$.

Há ainda o formalismo de interação, que é comumente usado quando o hamiltoniano é da forma $H=H_{0}+V(t)$ e conhecemos a solução para $H_{0}$. Definimos o vetor de estado no formalismo de interação como $\left|\psi_{I}(t)\right\rangle=U_{0}^{\dagger}(t)|\psi(t)\rangle$, com $U_{0}(t)=\exp \left[-(i / \hbar) H_{0} t\right]$. A equação se Schrödinger para $\left|\psi_{I}(t)\right\rangle$ é

$$
\begin{array}{r}
i \hbar \frac{\partial}{\partial t}\left|\psi_{I}(t)\right\rangle=V_{I}(t)\left|\psi_{I}(t)\right\rangle, \\
V_{I}(t)=U_{0}^{\dagger}(t) V(t) U_{0}(t) .
\end{array}
$$

No formalismo de interação, a evolução temporal atua tanto no vetor de estado quanto no nos operadores, por isso é tido como um formalismo intermediário. Ele é bastante usado em expansões perturbativas e em física atômica em geral, como será visto mais adiante.

\subsection{Operador Densidade}

Em muitos casos, nos deparamos com sistemas em que não é conhecido o estado inicial. Para lidar com problemas desse tipo no contexto quântico, usamos a idéia de operador densidade. Considere que, apesar de não saber em que estado se encontra o sistema, conhecemos a probabilidade, $p_{i}$, de que o mesmo esteja no estado $\left|\psi_{i}(0)\right\rangle$. Este $p_{i}$ será um peso estatístico diferente do mostrado em (2.7), pois este é fruto de uma ignorância do estado do sistema, que por alguma razão não pode ser esclarecida, e aquele aparece mesmo quando temos conhecimento completo sobre o estado do sistema. Sendo assim, a média de um operador $\hat{O}$ será a média, usando $p_{i}$ como peso, das médias nos vetores de estado possíveis,

$$
\langle\hat{O}\rangle=\sum_{i} p_{i}\left\langle\psi_{i}(t)|\hat{O}| \psi_{i}(t)\right\rangle
$$

que já engloba as médias quântica, no sentido de (2.5), e estatística. 
Se introduzimos a relação (2.4) na expressão anterior chegamos a

$$
\begin{aligned}
\langle\hat{O}\rangle & =\operatorname{Tr}[\rho(t) \hat{O}] \\
\rho(t) & =\sum_{i} p_{i}\left|\psi_{i}(t)\right\rangle\left\langle\psi_{i}(t)\right|
\end{aligned}
$$

onde $\rho(t)$ é operador densidade e (2.13) é a definição do mesmo ${ }^{1}$. Podemos escrever (2.13) como

$$
\rho(t)=U(t) \rho(0) U^{\dagger}(t)
$$

Ao diferenciar $\rho(t)$ chegamos á equação de Von Neumann,

$$
i \hbar \frac{d \rho(t)}{d t}=[H, \rho(t)]
$$

que fornece evolução temporal do operador densidade.

Note que há uma diferença de sinal entre esta equação e (2.8). Pelo fato de $\rho(t)$ ser dependente do tempo no formalismo de Schrödinger, ele não irá satisfazer a equação (2.8). Por substiuição direta, podemos mostrar que no formalismo de Heisenberg o operador densidade será constante.

Se usamos (2.12) para calcular a média do operador identidade, então

$$
\operatorname{Tr}[\rho(t)]=\sum_{i} p_{i}\left\langle\psi_{i}(t) \mid \psi_{i}(t)\right\rangle=\sum_{i} p_{i}
$$

portanto, a condição de normalização da probabilidade equivale a

$$
\operatorname{Tr}[\rho(t)]=1
$$

Ao calcular traço de $\rho^{2}(t)$ obtemos que $\operatorname{Tr}\left[\rho^{2}(t)\right]=\sum_{i, j} p_{i} p_{j}\left|\left\langle\psi_{i}(t) \mid \psi_{j}(t)\right\rangle\right|^{2}$. Caso os vetores sejam ortonormais, $\left\langle\psi_{i}(t) \mid \psi_{j}(t)\right\rangle=\delta_{i j}{ }^{2}$

$$
\operatorname{Tr}\left[\rho^{2}(t)\right]=\sum_{i} p_{i}^{2} \leq 1
$$

De modo que a igualdade ocorre somente no caso em que $p_{n}=1$, ou seja, o sistema está em um estado $\left|\psi_{n}(t)\right\rangle$ conhecido. A expressão (2.16) é utilizada para separar os estados puros das misturas. Um estado puro é aquele em que $\operatorname{Tr}\left[\rho^{2}(t)\right]=1$ e um estado mistura ocorre caso em que esta condição não seja satisfeita. O estado com máxima mistura é aquele em

\footnotetext{
${ }^{1}$ Esta derivação foi feita usando um espectro discreto, mas todos os resultados valem para o caso contínuo.

${ }^{2}$ Caso relação $\left\langle\psi_{i}(t) \mid \psi_{j}(t)\right\rangle \neq \delta_{i j}$ não seja válida, podemos sempre diagonalizar $\rho^{2}(t)$ e fazer a demonstração nesta base diagonal. Como o traço é invariante por mudança de base, isto completa a demonstração.
} 
que $p_{n}=1 / n$, onde $n$ é o tamanho espectro do sistema, portanto, $\operatorname{Tr}\left[\rho^{2}(t)\right]=1 / n$. Este operador densidade é diagonal em qualquer base, pois é múltiplo do operador identidade.

O operedor densidade pode ser expandido em uma base em que este não seja, em princípio, diagonal,

$$
\rho(t)=\sum_{i j} \rho_{i j}(t)|i\rangle\langle j|
$$

Os termos diagonais, $\rho_{i i}(t)$, são chamados de populações, pois fornecem a probabilidade de o sistema estar no estado $|i\rangle$ no tempo $t$. As componentes não diagonais são chamadas coerências, porque elas guardam a informação de quão puro é o estado descrito pelo operador densidade. Para o estato mais misturado possível as coerências são todas nulas em qualquer base, este operador densidade pode ser pensado como totalmente incoerente.

No formalismo de interação, $\rho_{I}(t)$ evolui como $V_{I}(t)$ em $(2.11)$, o que pode ser mostrado substituindo $\left|\psi_{i}(t)\right\rangle$ por $\left|\psi_{i I}(t)\right\rangle$ em (2.13). Supondo $H=H_{0}+V(t)$, a equação de Von Neumann para $\rho_{I}(t)$, com $V_{I}(t)$ sendo o mesmo que em $(2.11)$, se torna

$$
\begin{aligned}
i \hbar \frac{d \rho_{I}(t)}{d t} & =\left[V_{I}(t), \rho_{I}(t)\right], \\
\rho_{I}(t) & =U_{0}^{\dagger}(t) \rho(t) U_{0}(t) .
\end{aligned}
$$

\subsection{Relações de Incerteza e Flutuações Quânticas}

Pelo fato de se tratar de uma teoria intrinsecamente probabilística, é de se esperar que na Mecânica Quântica haja flutuações nas medidas mesmo desprezando o erro experimental e considerando um sistema puro. Nesta seção, faremos uma demonstração das relações de incerteza suponto o sistema em um estado puro. Definimos as flutuações do operador $\hat{A}$ como sendo o desvio quadrático médio,

$$
\Delta \hat{A}=\sqrt{\left\langle(\delta \hat{A})^{2}\right\rangle}
$$

onde $\delta \hat{A}=\hat{A}-\langle\hat{A}\rangle$ e as médias são feitas sobre vetores de estado. Considere os operadores hermitianos $\hat{A}$ e $\hat{B}$. Definimos $\left|\phi_{A}\right\rangle=\delta \hat{A}|\psi\rangle$ e $\left|\phi_{B}\right\rangle=\delta \hat{B}|\psi\rangle$, tal que

$$
(\Delta \hat{A} \Delta \hat{B})^{2}=\left\langle\phi_{A} \mid \phi_{A}\right\rangle\left\langle\phi_{B} \mid \phi_{B}\right\rangle
$$

Como pela desigualdade de Schwarz, $\left\langle\phi_{A} \mid \phi_{A}\right\rangle\left\langle\phi_{B} \mid \phi_{B}\right\rangle \geq\left|\left\langle\phi_{A} \mid \phi_{B}\right\rangle\right|^{2}$, podemos escrever

$$
(\Delta \hat{A} \Delta \hat{B})^{2} \geq\left|\left\langle\phi_{A} \mid \phi_{B}\right\rangle\right|^{2}=\frac{1}{4}|\langle\psi \mid[\delta \hat{A}, \delta \hat{B}]+\{\delta \hat{A}, \delta \hat{B}\} \| \psi\rangle|^{2},
$$


onde $\{$,$\} representa o anticomutador e como [\delta \hat{A}, \delta \hat{B}]=[\hat{A}, \hat{B}]$, chegamos à expressão para o princípio da incerteza,

$$
(\Delta \hat{A} \Delta \hat{B})^{2} \geq \frac{1}{4}|\langle[\hat{A}, \hat{B}]\rangle|^{2}+\frac{1}{4}|\langle\{\delta \hat{A}, \delta \hat{B}\}\rangle|^{2} .
$$

Ao olhar para esta expressão ficamos tentados a supor que quando tomamos a igualdade isto resultaria na menor flutuação quântica possível. Porém certo cuidado deve ser tomado ao realizar este procedimento. Se fizessemos isto, o valor mínimo da flutuação em (2.19) valeria para qualquer estado físico. Mas é razoável supor que existam estados especiais em que a incerteza seja a menor possível. O segundo termo de (2.19) é a covariância simetrizada de $\hat{A} \operatorname{com} \hat{B}$. Este termo, ao contrário do primeiro, não se anula ao tomar o limite clássico. Sendo assim, a menor flutuação quântica possivel é aquela que se anula no limite clássico, ou seja, resultado apenas do primeiro termo de (2.19),

$$
(\Delta \hat{A} \Delta \hat{B})^{2} \geq \frac{1}{4}|\langle\psi|[\hat{A}, \hat{B}]| \psi\rangle|^{2} .
$$

Supor que $|\langle\{\delta \hat{A}, \delta \hat{B}\}\rangle|=0$ é equivalente a impor uma condição para os estados que satisfazem o mínimo da relação de incerteza [30, 31]. Como

$$
\langle\delta \hat{A} \delta \hat{B}\rangle+\langle\delta \hat{B} \delta \hat{A}\rangle=0
$$

$\langle\delta \hat{A} \delta \hat{B}\rangle=\left\langle\phi_{A} \mid \phi_{B}\right\rangle$ e $\langle\delta \hat{B} \delta \hat{A}\rangle=\left\langle\phi_{B} \mid \phi_{A}\right\rangle$, então $\left\langle\phi_{A} \mid \phi_{B}\right\rangle=i \lambda$, onde $\lambda$ é real. Portanto, chegamos à seguinte condição para que o termo de covariância se anule,

$$
\left|\phi_{A}\right\rangle=i \lambda\left|\phi_{B}\right\rangle
$$

que pode ser expressa como

$$
(\hat{A}-i \lambda \hat{B})|\psi\rangle=(\langle\hat{A}\rangle-i \lambda\langle\hat{B}\rangle)|\psi\rangle
$$

Esta relação é satisfeita para os estados de mínima incerteza [31, 32]. Se fazemos $\hat{A}=\hat{q}$, $\hat{B}=\hat{p}$ e $\lambda=1$, onde $\hat{q}$ e $\hat{p}$ são, respectivamente, posição e momento, podemos escrever (2.21) como

$$
\hat{a}|\alpha\rangle=\alpha|\alpha\rangle
$$

onde $\hat{a}=(\hat{q}+i \hat{p}) / \sqrt{2}$. Os estados $|\alpha\rangle$ que satisfazem esta relação formam uma classe de estados de mínima incerteza chamada de estados coerentes. Eles são muito usados em ótica quântica e serão tópico de uma outra seção. 


\subsection{Teorema de Wigner-Eckart para Operadores Veto- riais}

Nesta seção apresentaremos o teorema de Wigner-Eckart para operadores vetoriais. Este teorema nos permite cálcular valores esperados de operadores da forma $\left\langle\alpha^{\prime} j^{\prime} m^{\prime}|\hat{O}| \alpha j m\right\rangle$ para uma certa classe de operadores chamados de operadores vetoriais, onde $j$ e $m$ caracterizam os autoestados de momento angular e $\alpha$ representa o conjunto de operadores compatíveis com o mesmo. O teorema será útil para fazer os cálculos do capítulo 5 .

Classicamente, um vetor $X$ é definido como um objeto invariante por transformação de coordenadas. Então, se a base do sistema de coordenadas se transforma como $e_{i}^{\prime}=$ $\sum_{j} \Lambda_{i j}^{-1} e_{j}$, as componentes de $X=\sum_{i} X_{i} e_{i}$, irão se transformar de forma contrária para manter o vetor constante,

$$
X_{i}^{\prime}=\sum_{j} \Lambda_{i j} X_{j}
$$

onde $\Lambda$ é a matriz de transformação. Caso $X$ seja o estado clássico no espaço de fase, suas componentes se transformarão da mesma forma que as do vetor posição. No caso mais específico em que a origem do sistema de coordenadas é fixada, as transformações serão rotações em torno desta origem ${ }^{3}$.

Na Mecânica Quântica, as rotações também são feitas sobre o vetor de estado, $|\psi\rangle$, ou seja,

$$
\left|\psi^{R}\right\rangle=D(R)|\psi\rangle
$$

onde $\left|\psi^{R}\right\rangle$ é o vetor transformado e $D(R)$ é o operador de rotação, definido como [29, 27]

$$
D(R)=e^{-\frac{i}{\hbar}(\hat{\mathbf{n}} \cdot \mathbf{J}) \theta},
$$

tal que $\mathbf{J}$ é o operador momento angular e $R$ significa a rotação de um ângulo $\theta$ em torno do eixo definido por $\hat{\mathbf{n}}$.

Se calcularmos o valor esperado de um operador $A$ no estado $\left|\psi^{R}\right\rangle$, obtemos que $\left\langle\psi^{R}|A| \psi^{R}\right\rangle \equiv\left\langle\psi\left|A^{R}\right| \psi\right\rangle$, onde definimos

$$
A^{R}=D^{\dagger}(R) A D(R) .
$$

\footnotetext{
${ }^{3}$ No ponto de vista das transformações ativas, em que o objeto físico é transformado em vez do sistema de coordenadas, podemos definir um vetor como um objeto de se transforma como o vetor posição. A transformação nas compenentes será essencialmente a mesma que nas transformações passivas, quando mudamos o sistema de coordenadas, porém com o ângulo de rotação $\theta \longrightarrow-\theta$. Para as rotações no espaço de Hilbert usaremos o ponto de vista ativo.
} 
Sendo assim, como na evolução temporal, podemos efetuar a rotação no operador e calcular os valores esperados nos estados não transformados. Para uma rotação infinitesimal, $\operatorname{com} \delta \theta<<1$,

$$
D(\delta R)=1-i \frac{\delta \theta(\hat{\mathbf{n}} \cdot \mathbf{J})}{\hbar} .
$$

Um vetor clássico se transforma por rotação infinitesimal como $X_{i}^{\prime}=X_{i}+\delta \theta(\hat{\mathbf{n}} \times \mathbf{X})_{i}$ [33]. Portanto, definimos um operador vetorial no contexto quântico como um objeto que se transforma por rotação como um vetor clássico,

$$
D^{\dagger}(\delta R) V_{i} D(\delta R)=V_{i}+\frac{i}{\hbar} \delta \theta \sum_{j} n_{j}\left[J_{j}, V_{i}\right]=V_{i}+\delta \theta \epsilon_{i j k} n_{j} V_{k}
$$

onde $\epsilon_{i j k}$ é o tensor de Levi-Civita, portanto,

$$
\left[V_{i}, J_{j}\right]=i \hbar \epsilon_{i j k} V_{k}
$$

Como pode ser visto por simples verificação, essa igualdade é satisfeita pelos operadores posição, momento e momento angular orbital.

Apesar de (2.26) ser um resultado muito importante e, muitas vezes, usado como definição dos operadores vetoriais, todo o potencial dos mesmos fica mais evidente quando usados na base esférica,

$$
\begin{aligned}
V_{ \pm 1} & =\mp \frac{1}{\sqrt{2}}\left(V_{x} \pm i V_{y}\right), \\
V_{0} & =V_{z} .
\end{aligned}
$$

Na qual as relações de comutação com $J_{z}$ e os operadores de subida e descida, definidos como $J_{ \pm}=J_{x} \pm i J_{y}$, são

$$
\begin{aligned}
{\left[J_{z}, V_{\mu}\right] } & =\hbar \mu V_{\mu} \\
{\left[J_{ \pm}, V_{\mu}\right] } & =\hbar a_{ \pm}(1 \mu) V_{\mu \pm 1}
\end{aligned}
$$

onde $\mu=-1,0,+1$ e $a_{ \pm}(j m)=\sqrt{j(j+1)-m(m \pm 1)}$. Ao aplicar $\left\langle j^{\prime} m^{\prime}\right|$ e $|j m\rangle$ em (2.29) e (2.30) obtemos

$$
\begin{aligned}
\left(m^{\prime}-m\right)\left\langle j^{\prime} m^{\prime}\left|V_{\mu}\right| j m\right\rangle= & \mu\left\langle j^{\prime} m^{\prime}\left|V_{\mu}\right| j m\right\rangle, \\
a_{\mp}\left(j^{\prime} m^{\prime}\right)\left\langle j^{\prime} m^{\prime} \mp 1\left|V_{\mu}\right| j m\right\rangle= & a_{ \pm}(j m)\left\langle j^{\prime} m^{\prime}\left|V_{\mu}\right| j m \pm 1\right\rangle \\
& +a_{ \pm}(1 \mu)\left\langle j^{\prime} m^{\prime}\left|V_{\mu \pm 1}\right| j m\right\rangle .
\end{aligned}
$$

As expressões anteriores são as mesmas satisfeitas pelos coeficientes de Clebsch- 
Gordan (CG), $\left\langle j^{\prime} m^{\prime} \mid j 1 m \mu\right\rangle[29,27,34,26]$. Note que a equação (2.31) fornece a regra de seleção $m^{\prime}=m+\mu$ e (2.30) fornece a desigualdade triangular,

$$
|j-1| \leq j^{\prime} \leq j+1
$$

além da relação de recursividade. Estas tambéms são as condições que devem ser satisfeitas para que os CG sejam não-nulos. Sendo assim, podemos fazer a igualdade

$$
\left\langle\alpha^{\prime} j^{\prime} m^{\prime}\left|V_{\mu}\right| \alpha j m\right\rangle=\left\langle\alpha^{\prime} j^{\prime}\|V\| \alpha j\right\rangle\left\langle j^{\prime} m^{\prime} \mid j 1 m \mu\right\rangle
$$

onde $\left\langle\alpha^{\prime} j^{\prime}\|V\| \alpha j\right\rangle$ é o elemente de matriz reduzido. Ele não depende da parte geométrica, definida por $m$ e $m^{\prime}$, e será proporcional ao próprio valor esperado calculado em uma base conveniente. Em boa parte dos casos, onde a parte geométrica é de maior interesse, este pode ser tratado como uma constante de proporcionalidade que pode ser medida. Uma expressão fechada para o elemento de matriz reduzido, assim como uma demonstração rigorosa do teorema, podem ser encontradas em [27]. A expressão (2.33) é versão para operadores vetoriais do teorema de Wigner-Eckart.

\subsection{Decomposição em Modos Normais}

A partir desta seção introduziremos alguns conceitos básicos de Ótica Quântica que serão importantes para o desenvolvimento deste trabalho, iniciando pela decomposição espectral do campo eletromagnético e pela quantização do mesmo. No eletromagnetismo clássico, o campo eletromagnético obedece às equações de Maxwell, que em unidades gaussianas são

$$
\begin{aligned}
\nabla \cdot \mathbf{B} & =0 \\
\nabla \times \mathbf{E} & =-\frac{1}{c} \frac{\partial \mathbf{B}}{\partial t}, \\
\nabla \cdot \mathbf{E} & =4 \pi \rho, \\
\nabla \times \mathbf{B} & =\frac{4 \pi \mathbf{J}}{c}+\frac{1}{c} \frac{\partial \mathbf{E}}{\partial t},
\end{aligned}
$$

onde $\mathbf{B}(x), \mathbf{E}(x), \mathbf{J}(x)$ e $\rho(x)$ são, respectivamente, o campo magnético, o campo elétrico e as densidades de corrente e carga, onde $x=(\mathbf{r}, t)$ representa a dependência espacial e temporal. Os campos podem ser expressos em termos dos potenciais vetor, $\mathbf{A}(x)$, e 
escalar, $\Phi(x)$, e as densidades de carga e corrente satisfazem a equação de continuidade,

$$
\begin{aligned}
\mathbf{E} & =-\frac{1}{c} \frac{\partial \mathbf{A}}{\partial t}-\nabla \Phi, \\
\mathbf{B} & =\nabla \times \mathbf{A}, \\
\nabla \cdot \mathbf{J} & +\frac{\partial \rho}{\partial t}=0 .
\end{aligned}
$$

Como os campos não dependem dos potenciais, mas de derivadas dos mesmos, há uma liberdade na escolha destes, desde que os valores dos campos não se alterem. Esta liberdade se reflete nas transformaçõs de calibre, que conectam potenciais dos quais são derivados os mesmos campos,

$$
\begin{aligned}
& \mathbf{A} \longrightarrow \mathbf{A}^{\prime}+\nabla \Lambda \\
& \Phi \longrightarrow \Phi^{\prime}-\frac{1}{c} \frac{\partial \Lambda}{\partial t}
\end{aligned}
$$

Usaremos o calibre de Coulomb, $\nabla \cdot \mathbf{A}=0$. Ao contrário do calibre de Lorentz, o mesmo não mantém a covariância de Lorentz das equações de movimento ao se passar da formulação com campos para aquela com potenciais. Porém, ao considerar a interação com matéria não-relativística, esta covariância é quebrada de qualquer forma e o calibre de Coulomb tem a vantagem de fazer com que o processo de quantização seja bem mais simples. Neste calibre, os potenciais satisfazem as equações

$$
\begin{aligned}
{\left[\frac{1}{c^{2}} \frac{\partial^{2}}{\partial t^{2}}-\nabla^{2}\right] \mathbf{A}(x) } & =\frac{4 \pi}{c} \mathbf{J}_{T}, \\
\nabla^{2} \Phi & =-4 \pi \rho,
\end{aligned}
$$

$\operatorname{com} \mathbf{J}_{T}=\mathbf{J}-\nabla \Phi / 4 \pi$ e $\nabla \cdot \mathbf{J}_{T}=0$. A partir de (2.40), podemos mostrar que $\frac{\partial}{\partial t} \nabla \Phi=4 \pi \mathbf{J}$. Para o caso em que não há cargas nem correntes, $\mathbf{J}=0$ e $\rho=0$, então $\nabla \Phi=0$, portanto, podemos considerar, sem perda de generalidade, que $\Phi=0$. Sendo assim, a única equação a ser considerada será

$$
\left[\frac{1}{c^{2}} \frac{\partial^{2}}{\partial t^{2}}-\nabla^{2}\right] A_{i}(x)=0 .
$$

Fazendo a separação de variáveis, $A_{i}(x)=A_{i}^{s}(\mathbf{r}) A_{i}^{t}(t)$, a última equação pode ser escrita como

$$
\begin{gathered}
{\left[\nabla^{2}+k^{2}\right] A_{i}^{s}(\mathbf{r})=0,} \\
\ddot{A}_{i}^{t}(t)+\omega^{2} A_{i}^{t}(t)=0,
\end{gathered}
$$

onde $k^{2}=\mathbf{k} \cdot \mathbf{k}$ é a constante que surge da separação de variávies, $\omega=k c$ e $\mathbf{k}=\left(k_{1}, k_{2}, k_{3}\right)$, 
de modo que cada $k_{j}^{2}$ é a constante que aparece quanto separamos a função de $r_{j}$.

Quando os campos estão confinados numa cavidade tridimensional suposta, por simplicidade, como um paralelepípedo de arestas $\left(L_{1}, L_{2}, L_{3}\right)$, podemos mostrar, pelas condições de contorno para $\mathbf{E}$ e $\mathbf{B}$ na fronteira, que o vetor $\mathbf{k}$ terá um espectro discreto dado por

$$
\mathbf{k}=\pi\left(\frac{n_{1}}{L_{1}}, \frac{n_{2}}{L_{2}}, \frac{n_{3}}{L_{3}}\right)
$$

onde $n_{1}, n_{2}, n_{3} \in \mathbb{Z}$. Isto significa que em cada dimensão cabe um múltiplo de $\lambda_{i} / 2$, com $\lambda_{i}=2 \pi / k_{i}$ e $\lambda=\sqrt{\lambda_{1}^{2}+\lambda_{2}^{2}+\lambda_{2}^{2}}$ sendo o comprimento de onda. O espaço formado pelos $k_{i}^{\prime} s$ é chamado de espaço recíproco e mais detalhes sobre a solução para $\mathbf{E}$ e $\mathbf{B}$ podem se vistos em [35].

Para o campo livre, que pode ser pensado como o campo contido numa cavidade de tamanho infinito, é de se esperar que o espectro seja contínuo. Para tratar o espaço livre de forma análoga a uma cavidade, vamos usar condições periódicas de contorno. Para isso, supomos o espaço dividido em caixas, mas, em vez de considerar as mesmas condições de contorno para os campos na cavidade, faremos a suposição mais fraca que estes sejam periódicos na fronteira, $A_{i}\left(r_{1}, r_{2}, r_{3}, t\right)=A_{i}\left(r_{1}+L_{1}, r_{2}, r_{3}, t\right)$, sendo que o mesmo vale para as outras dimensões. Essa idéia é a mesma aplicada em estado sólido para uma rede cristalina, que também corresponde a passar para o espaço recíproco, mas com a diferença que aqui sempre podemos fazer o volume da caixa arbitrariamente grande e recuperar o espectro contínuo. Neste caso, caberão múltiplos de $\lambda_{i}$ em vez de $\lambda_{i} / 2$ na i-ésima dimensão, o que pode ser visto por simples verificação, portanto,

$$
\mathbf{k}=2 \pi\left(\frac{n_{1}}{L_{1}}, \frac{n_{2}}{L_{2}}, \frac{n_{3}}{L_{3}}\right)
$$

e $A_{i}^{s}(\mathbf{r})=\bar{A}_{i} \exp [i \mathbf{k} \cdot \mathbf{r}]$. Pelo princípio da superposição, escrevemos a solução geral como

$$
A_{i}(x)=\sum_{\mathbf{k}} A_{\mathbf{k} i}(t) e^{i \mathbf{k} \cdot \mathbf{r}}
$$

de modo que as constantes $\bar{A}_{i}$ foram incorporadas, a soma em $\mathbf{k}$ é feita sobre todos os $n_{i}^{\prime} s$ e o índice $t$ em (2.45) foi suprimido para não carregar a notação ${ }^{4}$. Essa solução equivale a uma expansão do potencial vetor em série de Fourrier.

\footnotetext{
${ }^{4}$ Em princípio, poderíamos resolver a equação (2.45) e escrever explicitamete o fator $e^{-i \omega t}$. Porém, ao carregar a dependência temporal em $\mathbf{A}(t)$ chegaremos, após o processo de quantização, a operadores no formalismo de Heisenberg. Para o campo livre, ambas abordagens são completamente equivalentes. Porém, quando introduzimos uma interação que afeta apenas a dependência temporal, como é o caso do problema tratado neste trabalho, trabalhar no formalismo de Heisenberg se torna mais interessante. Porque o processo de quantização feito com a dependência explícita no tempo é válido tanto para o campo livre quanto para o caso em que haja interação.
} 
Como $A_{i}(x)$ deve ser uma grandeza real, somaremos à expressão (2.47) o seu complexo conjugado e esse será o novo $A_{i}(x)$, então

$$
\mathbf{A}(x)=\sum_{\mathbf{k}}\left[\mathbf{A}_{\mathbf{k}}(t) e^{i \mathbf{k} \cdot \mathbf{r}}+\text { c.c. }\right]
$$

Mais detalhes sobre esta expansão podem ser vistos em [36, 27]. Aplicando o calibre de Coulomb na expressão acima, obtemos

$$
\mathbf{k} \cdot \mathbf{A}_{\mathbf{k}}(t)=0
$$

ou seja, cada coeficiente $\mathbf{A}_{\mathbf{k}}(t)$ está definido no plano transverso ao vetor de propagação k, por isso este é conhecido como calibre transverso. Note que mesmo a equação de movimento com fontes, (2.42), está definida no espaço transverso [37, 38]. Podemos definir uma base $\left(\mathbf{e}_{\mathbf{k} 1}, \mathbf{e}_{\mathbf{k} 2}, \mathbf{k}_{u}\right)$, onde $\mathbf{k}_{u}=\mathbf{k} / k$ e $\mathbf{e}_{\mathbf{k} \lambda}$ são dois vetores unitários no plano transverso com $\lambda=1,2$. Desta forma,

$$
\mathbf{A}_{\mathbf{k}}(t)=\sum_{\lambda=1}^{2} A_{\mathbf{k} \lambda}(t) \mathbf{e}_{\mathbf{k} \lambda} .
$$

Os vetores $\mathbf{e}_{\mathbf{k} \lambda}$ definem a polarização do campo e cada componente $A_{\mathbf{k} \lambda}(t)$ será chamada de modo do campo. Neste sentido, existem modos de frequência, definidos por $k$, de direção, dados pela diferentes combinações dos $n_{i}^{\prime} s$, e de polarização, para cada $\lambda$. A base transversa a ser escolhida pode ser linear, circular ou qualquer combinação das duas. Usando (2.38) e (2.39), podemos mostrar que

$$
\begin{aligned}
& \mathbf{E}(x)=i \sum_{\mathbf{k} \lambda}\left[k A_{\mathbf{k} \lambda}(t) \hat{e}_{\mathbf{k} \lambda} e^{i \mathbf{k} \cdot \mathbf{r}}-c . c .\right] \\
& \mathbf{B}(x)=i \sum_{\mathbf{k} \lambda}\left[\left(\mathbf{k} \times \hat{e}_{\mathbf{k} \lambda}\right) A_{\mathbf{k} \lambda}(t) e^{i \mathbf{k} \cdot \mathbf{r}}-c . c .\right]
\end{aligned}
$$

O hamiltoniano para o campo eletromagnético livre é [39]

$$
H_{f}=\frac{1}{8 \pi} \int_{V} d^{3} r\left(\mathbf{E}^{2}+\mathbf{B}^{2}\right)
$$

onde $V=L_{1} L_{2} L_{2}$. Substituindo as expressões (2.50)-(2.51) e usando a seguinte propriedade,

$$
\int_{V} d^{3} r e^{i\left(\mathbf{k}-\mathbf{k}^{\prime}\right) \cdot \mathbf{r}}=V \delta_{\mathbf{k k}^{\prime}},
$$

que pode ser mostrada calculando o lado esquerdo em coordenadas cartesianas, chegamos 
à expressão para o hamiltoniano no espaço recíproco,

$$
H_{f}=\frac{V}{2 \pi} \sum_{\mathbf{k} \lambda} k^{2}\left|A_{\mathbf{k} \lambda}(t)\right|^{2} .
$$

\subsection{Quantização do Campo Eletromagnético Livre}

Podemos partir deste hamiltoniano para fazer a quantização do campo eletromagnético [27, 40, 41, 36, 42]. Definimos as quantidades

$$
\begin{aligned}
p_{\mathbf{k} \lambda}(t) & =\sqrt{\frac{k V}{\pi \hbar c}} \operatorname{Im}\left(A_{\mathbf{k} \lambda}(t)\right), \\
q_{\mathbf{k} \lambda}(t) & =\sqrt{\frac{k V}{\pi \hbar c}} \operatorname{Re}\left(A_{\mathbf{k} \lambda}(t)\right),
\end{aligned}
$$

de modo que (2.53) se torna

$$
H_{f}=\sum_{\mathbf{k} \lambda} \frac{\hbar \omega}{2}\left[p_{\mathbf{k} \lambda}^{2}+q_{\mathbf{k} \lambda}^{2}\right],
$$

que é o hamiltoniano do conjunto de osciladores harmômicos desacoplados com momento e posição adimensionais dados por $p_{\mathbf{k} \lambda}$ e $q_{\mathbf{k} \lambda}$. O próximo passo é impor a quantização canônica para estas variáveis, tal que

$$
\left[\hat{q}_{\mathbf{k} \lambda}(t), \hat{p}_{\mathbf{k}^{\prime} \lambda^{\prime}}(t)\right]=i \delta_{\mathbf{k k}^{\prime}} \delta_{\lambda \lambda^{\prime}}
$$

e estes passem a ser operadores atuando no espaço de Hilbert. Esta relação de comutação é a mesma que iríamos encontrar ao calcularmos a densidade lagrangiana, a densidade de momento canônico, $\Pi_{i}(x)$, e impor a quantização canônica para campos ${ }^{5},\left[A_{i}(x), \Pi_{i}\left(x^{\prime}\right)\right]=$ $i \hbar \delta\left(\mathbf{r}-\mathbf{r}^{\prime}\right) \delta\left(t-t^{\prime}\right)[38,37]$.

Definimos os operadores de criação e destruição para cada modo do campo como

$$
\begin{aligned}
\hat{a}_{\mathbf{k} \lambda}(t) & =\frac{1}{\sqrt{2}}\left[\hat{q}_{\mathbf{k} \lambda}(t)+i \hat{p}_{\mathbf{k} \lambda}(t)\right], \\
\hat{a}_{\mathbf{k} \lambda}^{\dagger}(t) & =\frac{1}{\sqrt{2}}\left[\hat{q}_{\mathbf{k} \lambda}(t)-i \hat{p}_{\mathbf{k} \lambda}(t)\right], \\
{\left[\hat{a}_{\mathbf{k} \lambda}(t), \hat{a}_{\mathbf{k}^{\prime} \lambda^{\prime}}^{\dagger}(t)\right] } & =\delta_{\mathbf{k k}^{\prime}} \delta_{\lambda \lambda^{\prime}} .
\end{aligned}
$$

\footnotetext{
${ }^{5}$ Como estamos no calibre de Coulomb e $\Phi=0$, a relação de comutação está definida apenas para as componentes espaciais.
} 
Podemos ver que a equivalência no processo de quantização será

$$
\begin{aligned}
& A_{\mathbf{k} \lambda}(t) \longrightarrow \sqrt{\frac{2 \pi \hbar c}{k V}} \hat{a}_{\mathbf{k} \lambda}(t), \\
& A_{\mathbf{k} \lambda}^{*}(t) \longrightarrow \sqrt{\frac{2 \pi \hbar c}{k V}} \hat{a}_{\mathbf{k} \lambda}^{\dagger}(t) .
\end{aligned}
$$

Ao final do processo os operadores já aparecem no formalismo de Heisenberg. Aplicando a equação de Heisenberg para $a_{\mathbf{k} \lambda}(t)$, chegamos à equação $\dot{\hat{a}}_{\mathbf{k} \lambda}(t)=-i \omega \hat{a}_{\mathbf{k} \lambda}(t)$, portanto,

$$
\hat{a}_{\mathbf{k} \lambda}(t)=\hat{a}_{\mathbf{k} \lambda} e^{-i \omega t}
$$

Deste modo, os campos quantizados no espaço livre passam a ter a forma conhecida,

$$
\begin{aligned}
& \hat{\mathbf{A}}(x)=\sum_{\mathbf{k} \lambda}\left[\sqrt{\frac{2 \pi \hbar c}{k V}} \hat{a}_{\mathbf{k} \lambda} \mathbf{e}_{\mathbf{k} \lambda} e^{i(\mathbf{k} \cdot \mathbf{r}-\omega t)}+h . c .\right], \\
& \hat{\mathbf{E}}(x)=i \sum_{\mathbf{k} \lambda}\left[\sqrt{\frac{2 \pi \hbar k c}{V}} \hat{a}_{\mathbf{k} \lambda} \mathbf{e}_{\mathbf{k} \lambda} e^{i(\mathbf{k} \cdot \mathbf{r}-\omega t)}-h . c .\right], \\
& \hat{\mathbf{B}}(x)=i \sum_{\mathbf{k} \lambda}\left[\sqrt{\frac{2 \pi \hbar c}{k V}} \hat{a}_{\mathbf{k} \lambda}\left(\mathbf{k} \times \mathbf{e}_{\mathbf{k} \lambda}\right) e^{i(\mathbf{k} \cdot \mathbf{r}-\omega t)}-h . c .\right] .
\end{aligned}
$$

Como estes operadores dependem de $\hat{a}_{\mathbf{k} \lambda}$ e $\hat{a}_{\mathbf{k} \lambda}^{\dagger}$, a base mais natural para se expandir os autoestados do campo é a base de Fock ${ }^{6}$. Um campo com $N$ modos e $n_{i}$ fótons em cada modo $i$ é descrito como

$$
\left|\left\{n_{i}\right\}\right\rangle=\prod_{i}\left|n_{1}\right\rangle \otimes \ldots \otimes\left|n_{i}\right\rangle \otimes \ldots \otimes\left|n_{N}\right\rangle
$$

O número médio de fótons no modo $j$ é $n_{j}=\left\langle\left\{n_{i}\right\}\left|\hat{a}_{j}^{\dagger} \hat{a}_{j}\right|\left\{n_{i}\right\}\right\rangle$. O estado com $n_{j}=0$ é chamado estado de vácuo, pois possui número de fótons nulo e tem a propriedade que $\hat{a}_{j}|0\rangle=0$.

Como visto em (2.64), o operador campo elétrico pode ser escrito como $\hat{\mathbf{E}}(x)=$ $\hat{\mathbf{E}}^{(+)}(x)+\hat{\mathbf{E}}^{(-)}(x)$, em que $(+)$ e $(-)$ representam os termos que multiplicam as frequências positivas e negativas, respectivamente,

$$
\hat{\mathbf{E}}^{(+)}(x)=i \sum_{\mathbf{k} \lambda} \sqrt{\frac{2 \pi \hbar \omega}{V}} \hat{a}_{\mathbf{k} \lambda} e^{i(\mathbf{k} \cdot \mathbf{r}-\omega t)} \mathbf{e}_{\mathbf{k} \lambda},
$$

$\hat{\mathbf{E}}^{(-)}(x)=\left[\hat{\mathbf{E}}^{(+)}(x)\right]^{\dagger}$. Esta decomposição é similar à expressão (2.48). No espaço recíproco,

\footnotetext{
${ }^{6}$ Diferentemente do campo clássico, o estado do sistema será um vetor no espaço de Hilbert e não os campos propriamente ditos. Então, mesmo que o estado físico possua poucos modos, os operadores de campo devem ser utilizados, em princípio, sempre levando em consideração todos os modos.
} 
o hamiltoniano do campo livre se torna

$$
H_{f}=\sum_{\mathbf{k}, \lambda} \hbar \omega_{k}\left(\hat{a}_{\mathbf{k}, \lambda}^{\dagger} \hat{a}_{\mathbf{k}, \lambda}+\frac{1}{2}\right)
$$

Como enfatizado anteriormente, podemos sempre tomar o limite quando a caixa se torna infinita, que significa deixar que ondas com comprimento de onda maiores caibam dentro da caixa, ou seja, tomar o limite de espectro contínuo. Usaremos modos discretos ou contínuos conforme a conveniência, ficando claro que um sempre é o caso limite do outro. Da expressão (2.46), concluímos que $\prod_{i} \Delta k_{i}=\frac{(2 \pi)^{3}}{V}$, então

$$
\sum_{\mathbf{k}} \longrightarrow \frac{(2 \pi)^{3}}{V} \int_{V} d^{2} k,
$$

que é a regra utilizada para transformar a soma de modos numa integral no limite contínuo.

\subsection{Estados Coerentes}

Os estados coerentes possuem um papel importante na Mecânica Quântica em geral e principalmente na Ótica Quântica. Eles possuem diversas propriedades interessantes, dentre as quais duas são de grande importância dentro da ótica. A primeira é que eles são estados de mínima incerteza, podendo ser derivados de (2.21). A segunda propriedade importante, ressaltada por Glauber, é que as funções de correlação, que serão mostradas mais adiante, são diagonais se calculadas usando como vetor de estado um estado coerente $[43,44]$. Como mostrado em (2.22),

$$
\hat{a}|\alpha\rangle=\alpha|\alpha\rangle
$$

onde $|\alpha\rangle$ é o estado coerente definido pelo número complexo $\alpha$ e estamos nos restringindo a apenas um modo do campo. Ao multiplicar $\langle n-1|$ pela esquerda obtemos

$$
\langle n \mid \alpha\rangle=\frac{\alpha}{\sqrt{n}}\langle n-1 \mid \alpha\rangle .
$$

Realizando o procedimento recursivo, chegamos a

$$
\langle n \mid \alpha\rangle=\frac{\alpha^{n}}{\sqrt{n !}}\langle 0 \mid \alpha\rangle
$$

e, como $|\alpha\rangle=\sum_{n}\langle n \mid \alpha\rangle|n\rangle$, obtemos $|\alpha\rangle=\langle 0 \mid \alpha\rangle \sum_{n} \frac{\alpha^{n}}{\sqrt{n !}}|n\rangle$. Se impomos que $\langle\alpha \mid \alpha\rangle=1$, podemos mostrar que $|\langle 0 \mid \alpha\rangle|^{2}=\exp \left[-|\alpha|^{2}\right]$. Como há uma arbitrariedade de fase na 
escolha de $|\alpha\rangle$, escolhemos $\langle 0 \mid \alpha\rangle$ real, tal que

$$
|\alpha\rangle=e^{-\frac{1}{2}|\alpha|^{2}} \sum_{n} \frac{\alpha^{n}}{\sqrt{n !}}|n\rangle .
$$

O número médio de fótons calculado em um estado coerente é dado por $\bar{N}=\left\langle\alpha\left|\hat{a}^{\dagger} \hat{a}\right| \alpha\right\rangle$, que pode ser calculado usando (2.69),

$$
\begin{array}{r}
\left\langle\alpha\left|\hat{a}^{\dagger} \hat{a}\right| \alpha\right\rangle=\sum_{n} n p_{n}, \\
p_{n}=e^{-|\alpha|^{2}} \frac{|\alpha|^{n}}{n !} .
\end{array}
$$

Esta relação, junto com (2.69), mostram que o estado coerente é uma superposição de estados número e possui distribuição poissoniana de fótons. Esta ditribuição, no limite de $n$ grande, converge para uma gaussiana. Como a intensidade da luz é proporcional ao número de fótons, os estados coerentes não possui intensidade bem definida, diferentemente dos estados número. Porém, a fase destes estados não é completamente arbitária como a dos estados número. Por estes motivos, por ser um estado de mínima incerteza e por ser facilmente gerado em laboratório, o estado coerente é chamado de estado clássico do campo.

O estado coerente também pode ser obtido a partir da aplicação do operador deslocamento no estado no vácuo, $D(\alpha)|0\rangle$. Como

$$
|n\rangle=\frac{\left(\hat{a}^{\dagger}\right)^{n}}{\sqrt{n !}}|0\rangle,
$$

então $|\alpha\rangle=e^{-\frac{1}{2}|\alpha|^{2}} e^{\alpha \hat{a}^{\dagger}}|0\rangle$. Queremos que o operador aplicado no vácuo seja unitário, pois $\langle\alpha \mid \alpha\rangle=\langle 0 \mid 0\rangle=1$, porém o operador que obtemos claramente não é unitário. Mas como $|0\rangle=\exp \left[-\hat{a} \alpha^{*}\right]|0\rangle$, esta substituição irá garantir a unitariedade do operador resultante,

$$
|\alpha\rangle=e^{-\frac{1}{2}|\alpha|^{2}} e^{\alpha \hat{a}^{\dagger}} e^{-\alpha^{*} \hat{a}}|0\rangle .
$$

Por meio da forma reduzida do teorema BCH $[42,40]$ que diz que se dois operadores $\hat{A}$ e $\hat{B}$ são tais que $[\hat{A}, \hat{B}] \neq 0$, mas $[[\hat{A}, \hat{B}], \hat{B}]=[[\hat{A}, \hat{B}], \hat{A}]=0$, então

$$
e^{\hat{A}+\hat{B}}=e^{-\frac{1}{2}[\hat{A}, \hat{B}]} e^{\hat{A}} e^{\hat{B}}
$$

podemos escrever $D(\alpha)=\exp \left[\alpha \hat{a}^{\dagger}-\alpha^{*} \hat{a}\right]$ e o estado coerente pode ser visto como o vácuo deslocado,

$$
|\alpha\rangle=D(\alpha)|0\rangle .
$$


Outra forma para o operador deslocamento é $D(\alpha)=e^{\frac{1}{2}|\alpha|^{2}} e^{-\alpha^{*} \hat{a}} e^{\alpha \hat{a}^{\dagger}}$. Ele é chamado assim, pois gera um deslocamento tanto em $\hat{a}$ quanto em $\hat{a}^{\dagger}$,

$$
D^{\dagger}(\alpha) \hat{a} D(\alpha)=\hat{a}+\alpha
$$

Os estados coerentes também aparecem como a solução do problema do oscilador harmônico forçado quando o estado inicial é o estado de vácuo [28, 27].

\subsection{Operadores no Domínio da Frequência}

Nesta seção, estudaremos os operadores do campo no domínio da frequência, pois as medidas são obtidas neste domínio. Para o campo monomodo com frequência $\omega_{0}$,

$$
\begin{aligned}
\hat{a}(t) & =\hat{a} e^{-i \omega_{0} t}, \\
\hat{a}^{\dagger}(t) & =\hat{a}^{\dagger} e^{i \omega_{0} t},
\end{aligned}
$$

porém o campo fornecido por uma fonte laser não é estritamente monomodo, ainda que o seja nos valores médios ${ }^{7}$. Desta forma, escreveremos $\hat{a}(t)$ e $\hat{a}^{\dagger}(t)$ como resultado da soma sobre várias frequências,

$$
\begin{aligned}
\hat{a}(t) & =\int_{0}^{\infty} d \omega \hat{a}(\omega) e^{-i \omega t} \\
\hat{a}^{\dagger}(t) & =\int_{0}^{\infty} d \omega \hat{a}^{\dagger}(\omega) e^{i \omega t} .
\end{aligned}
$$

Os operadores $\hat{a}(\omega)$ e $\hat{a}^{\dagger}(\omega)$ satisfazem a seguinte regra de comutação $\left[\hat{a}(\omega), \hat{a}^{\dagger}\left(\omega^{\prime}\right)\right]=$ $2 \pi \delta\left(\omega-\omega^{\prime}\right)$, obtida tomando o limite contínuo de (2.59).

Como o laser oscila á frequência $\omega_{0}$, podemos fazer a substituição de variáveis $\omega=$ $\omega_{0} \pm \Omega$ nas equações acima e obter os operadores de campo em função dos operadores lentamente variáveis, $\hat{a}(t)=\hat{\tilde{a}}(t) e^{-i \omega_{0} t}$ e $\hat{a}^{\dagger}(t)=\hat{\tilde{a}}^{\dagger}(t) e^{i \omega_{0} t}$. Se as outras frequências que contribuem para o laser são tais que $\Omega \ll \omega_{0}$, o que é verdadeiro para um laser quase monomodo, podemos estender os limites de integração em $\omega_{0}$ até $\infty$ e em $-\omega_{0}$ a $-\infty$, tal que

$$
\begin{aligned}
\hat{\tilde{a}}(t) & =\int_{-\infty}^{\infty} d \Omega \hat{a}\left(\omega_{0}-\Omega\right) e^{i \Omega t}, \\
\hat{\tilde{a}}^{\dagger}(t) & =\int_{-\infty}^{\infty} d \Omega \hat{a}^{\dagger}\left(\omega_{0}+\Omega\right) e^{i \Omega t} .
\end{aligned}
$$

\footnotetext{
${ }^{7}$ Podemos escrever $\hat{a}(t)=[\alpha+\delta \hat{a}(t)] e^{-i \omega_{0} t}$, onde $\alpha=\langle\hat{a}\rangle$. Que significa um campo monomodo no valor médio, mas multimodo na flutuação.
} 
Faremos a seguinte definição, $\hat{\tilde{a}}(\Omega) \equiv \hat{a}\left(\omega_{0}-\Omega\right)$ e $\hat{\tilde{a}}^{\dagger}(\Omega) \equiv \hat{a}^{\dagger}\left(\omega_{0}+\Omega\right)$, pois estes operadores formam pares de trasnformadas de Fourier com $\hat{\tilde{a}}(t)$ e $\hat{\tilde{a}}^{\dagger}(t)$. Invertendo as relações $(2.71)$ e $(2.72)$, obtemos

$$
\begin{aligned}
\hat{\tilde{a}}(\Omega) & =\frac{1}{2 \pi} \int_{-\infty}^{\infty} d t \hat{\tilde{a}}(t) e^{-i \Omega t} \\
\hat{\tilde{a}}^{\dagger}(\Omega) & =\frac{1}{2 \pi} \int_{-\infty}^{\infty} d t \hat{\tilde{a}}^{\dagger}(t) e^{-i \Omega t}
\end{aligned}
$$

Note que $[\hat{\tilde{a}}(\Omega)]^{\dagger}=\hat{\tilde{a}}^{\dagger}(-\Omega)$ e vale a relação de comutação

$$
\left[a(\Omega), a^{\dagger}\left(\Omega^{\prime}\right)\right]=2 \pi \delta\left(\Omega+\Omega^{\prime}\right)
$$

Estes são os operadores de criação e destruição na frequência $\Omega$ deslocada da frequência central $\omega_{0}$. Estas frequências são chamadas de bandas laterais. Para um feixe coerente e monomodo, praticamente todos os fótons estarão na frequência central. Isto equivale a dizer que a média dos operadores de campo é nula para $\Omega \neq 0$. No caso em que isto ocorre, é mais simples obter a informação sobre as flutuações presentes nas bandas laterais. Considere que $\hat{\tilde{a}}(t)=\alpha+\delta \hat{\tilde{a}}(t)$, que equivale a um campo monomodo com valor médio $\alpha e^{-i \omega_{0} t}$. Portanto,

$$
\begin{aligned}
\hat{\tilde{a}}(\Omega) & =\alpha \delta(\Omega)+\delta \hat{\tilde{a}}(\Omega), \\
\delta \hat{a}(\Omega) & =\frac{1}{2 \pi} \int_{-\infty}^{\infty} d t \delta \hat{\tilde{a}}(t) e^{-i \Omega t}
\end{aligned}
$$

Neste caso, temos um valor médio infinito definido em $\Omega=0$ e nulo para qualquer outra frequência, mas a flutuação está distribuida para $\Omega \neq 0$ por meio da flutuação dependente do tempo $\delta \hat{\tilde{a}}(t)^{8}$. Por este motivo, informações sobre as flutuações de um sistema em variáveis contínuas são observadas em geral nas bandas laterais, medidas no domínio da frequência.

\subsection{Quadraturas do Campo Eletromagnético}

No eletromagnetismo clássico, podemos escrever as soluções para o campo livre como em (2.48), com o coeficiente de Fourier na forma

$$
A_{\mathbf{k}}(t)=\left|A_{\mathbf{k}}\right| e^{-i(\omega t-\phi)} .
$$

\footnotetext{
${ }^{8}$ Este resultado é similar ao obtido no apêndice $\mathrm{C}$ e é um caso particular o teorema de WienerKhinthchine.
} 
Para um único modo, $\mathbf{A}(x)=2\left|A_{\mathbf{k}}\right| \cos (\mathbf{k} \cdot \mathbf{r}-\omega t+\phi) \hat{e}_{\mathbf{k}}$. O campo pode ser descrito graficamente por meio de um plano complexo como na figura 2.1, onde $z=\left(\left|A_{\mathbf{k}}\right| / 2\right) \exp (i \alpha)$, $\alpha=\mathbf{k} \cdot \mathbf{r}-\omega t+\phi, \operatorname{Re}(z)=\left|A_{\mathbf{k}}\right| \cos (\alpha)$ e a parte imaginária de $z$ não possui sentido físico. Esta representação é conhecida como diagrama de Fresnel e é bastante usado no

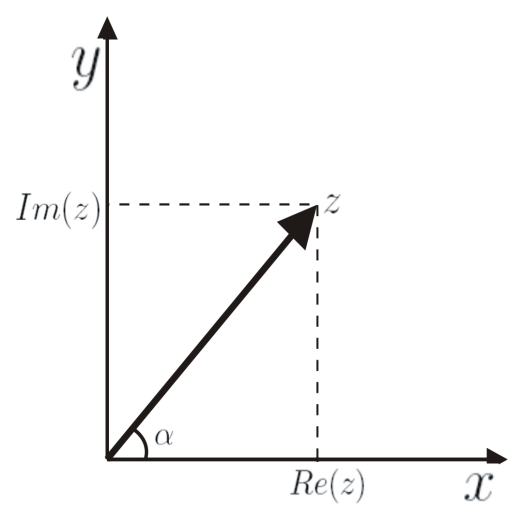

Figura 2.1: Diagrama de Fresnel para o campo clássico monomodo.

tratamento de circuitos elétricos, onde os campos oscilam apenas no tempo.

Para o caso do campo quantizado, a interpretação da parte real e imaginária do diagrama de Fresnel não é possível, pois o campo não pode ser expresso como um cosseno quando quantizado. Então, iremos reinterpretar $\operatorname{Re}(z)$ e $\operatorname{Im}(z)$ de maneira que o campo quantizado possa ser descrito em um diagrama de Fresnel. Se fazemos $z=A e^{-i \alpha}+A^{*} e^{i \alpha}=$ $X \cos (\alpha)+Y \sin (\alpha)$, onde $A \equiv A_{\mathbf{k}}, X=2 \operatorname{Re}(A)=A+A^{*}$ e $Y=2 \operatorname{Im}(A)=i\left(A^{*}+A\right), \mathrm{o}$ campo pode ser descrito no diagrama de Fresnel, porém não mais em um plano complexo, mas em um plano com duas dimensões reais. As quantidades $X$ e $Y$ são chamadas de quadraturas do campo eletromagnético. Se giramos nossa base de $\alpha$ na figura 2.1, $z \longrightarrow X=A+A^{*}$, ou seja, $X$ é a amplitude do campo, por isso será chamada de quadratura amplitude. Após a rotação, a quadratura $Y$ estará na direção perpendicular à $z$, por isso será chamada de quadratura fase. Note que as duas quadraturas estão definidas para um único modo do campo.

Para o caso do campo quantizado, em vez de combinações reais das amplitude de campo, podemos fazer combinações hermitianas dos operadores do campo,

$$
\begin{gathered}
\hat{X}=\hat{a}+\hat{a}^{\dagger}, \\
\hat{Y}=i\left(\hat{a}^{\dagger}-\hat{a}\right) .
\end{gathered}
$$

Estes operadores são os mesmos $\hat{p}_{\mathbf{k} \lambda}$ e $\hat{q}_{\mathbf{k} \lambda}$ definidos na secção anterior. Então o campo 
elétrico (2.50) se torna

$$
\hat{\mathbf{E}}(x)=\sum_{\mathbf{k} \lambda} \sqrt{\frac{2 \hbar k c}{\pi V}}\left[\hat{X}_{\mathbf{k} \lambda} \sin (-\omega t+\mathbf{k} \cdot \mathbf{r})-\hat{Y}_{\mathbf{k} \lambda} \cos (-\omega t+\mathbf{k} \cdot \mathbf{r})\right] \hat{e}_{\mathbf{k} \lambda} .
$$

Como para o campo clássico, os operadores quadratura de amplitude e fase do campo serão, respectivamente, $\hat{X}_{\mathbf{k} \lambda}$ e $\hat{Y}_{\mathbf{k} \lambda}$, cuja relação de comutação é

$$
\left[\hat{X}_{\mathbf{k} \lambda}, \hat{Y}_{\mathbf{k}^{\prime} \lambda^{\prime}}\right]=2 i \delta_{\mathbf{k k}^{\prime}} \delta_{\lambda \lambda^{\prime}}
$$

As quadraturas do campo são operadores incompatíveis cuja álgebra é a mesma dos operadores posição e momento. A partir daqui, nos restringiremos, por simplicidade, a um único modo do campo,

$$
\begin{gathered}
\hat{X}_{\phi}=\hat{a}^{\dagger} e^{i \phi}+\hat{a} e^{-i \phi}, \\
\hat{Y}_{\phi}=i\left(\hat{a}^{\dagger} e^{i \phi}-\hat{a} e^{-i \phi}\right),
\end{gathered}
$$

onde consideramos que $\mathbf{r}=0$ e $\phi=\omega t$. Portanto, $X_{\phi+\pi / 2}=Y_{\phi}$ e $Y_{\phi-\pi / 2}=X_{\phi}$. Neste sentido, eles são perpendiculares, como no caso do campo clássico, e podem ser usados como base para o diagrama de Fresnel.
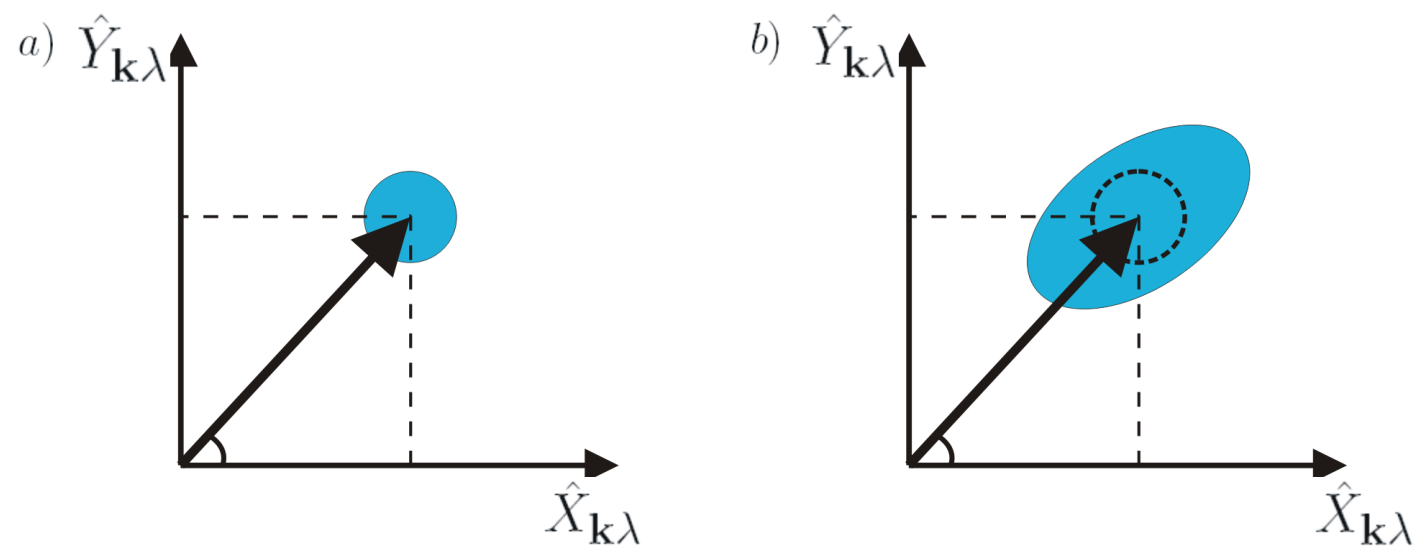

Figura 2.2: Diagrama de Fresnel para cada modo $\mathbf{k} \lambda$, a seta representa o valor médio e a mancha em torno da ponta desta, a flutuação. a) o campo está em um estado coerente. b) o campo com excesso de ruído em ambas quadraturas.

A intenside de um campo monomodo será proporcional ao número de fótons que atinge uma determinada área durante um intervalo de tempo,

$$
\hat{I}(t) \propto \hat{\tilde{a}}^{\dagger}(t) \hat{\tilde{a}}(t)
$$


Se fazemos $\hat{\tilde{a}}(t)=\alpha+\delta \hat{\tilde{a}}(t)$, então

$$
\begin{aligned}
\hat{I} & =|\alpha|^{2}+\delta \hat{I}, \\
\delta \hat{I}(t) & =|\alpha|\left[\delta \hat{a}(t) e^{i \phi}+\delta \hat{a}^{\dagger}(t) e^{-i \phi}\right],
\end{aligned}
$$

onde desprezamos o termo de segunda ordem. Como pode ser visto em $(2.77), \delta \hat{I}(t) \propto$ $\delta \hat{X}_{-\phi}(t)$. As flutuações na quadratura amplitude são obtidas medindo-se diretamente as flutuações de intensidade sempre que $\delta \hat{a}(t) \ll \alpha$. Podemos medir as flutuações na quadratura fase de um feixe projetanto-as nas flutuações da quadratura amplitude de outro por meio de processos de interferência como a deteção homodina, que será tratada no capítulo de métodos experimentais. Um operador hermitiano responsável pela fase do sistema ainda é assunto de discussão atualmente e a quadratura $\hat{Y}$ somente representa a fase do sistema no caso em que $\delta \hat{Y}(t) \ll|\alpha|[38,42,45]$.

Pelo fato de as quadraturas serem operadores incompatíveis, as relações de incerteza nos diz que ambas não podem ser conhecidas ao mesmo tempo com precisão arbitrária. Sendo assim, o campo elétrico será representado como um valor médio bem definido, que corresponde ao campo clássico, e uma mancha representando os diversos valores que este pode assumir, como na figura 2.2. Para os estados coerentes,

$$
\Delta X_{\phi}=\Delta Y_{\phi}=1
$$

que é o mínimo permitido pelo princípio da incerteza.

\subsection{Funções de Correlação}

Em geral, o que se mede nos experimentos de ótica quântica são intensidade luminosa, no regime contínuo, e contagem de fótons, no regime discreto. Para interpretar os resultados em ambos regimes, é preciso haver uma forma de associar o sinal obtido no detetor à teoria. Essa ligação foi proposta por Glauber na forma de funções de correlação [43].

O processo físico que ocorre em um detetor como um fotodiodo é o efeito fotoelétrico. Um fóton que atinge o detetor, situado em $\mathbf{r}$, no tempo $t$ é absorvido e um elétron é emitido. Neste caso, podemos considerar como hamiltoniano de interação o hamiltoniano de dipolo, descrito no apêndice A. Usando teoria de perturbação dependente do tempo, podemos mostrar que a probabilidade de transição do estado inicial $\left|\psi_{i}\right\rangle$ para o estado 
final $\left|\psi_{f}\right\rangle$ é dada por

$$
P_{i \rightarrow f} \sim\left|\left\langle\psi_{f}|V| \psi_{i}\right\rangle\right|^{2}
$$

onde $V=-\mathbf{d} \cdot \hat{\mathbf{E}}$, com $\hat{\mathbf{E}}$ definido em (2.64). Podemos expandir o estado do campo numa base de Fock e, se o processo que ocorre é a destruição de um fóton na polarização $\lambda$ e com momento $\mathbf{k}$, os estados inicial e final são $\left|\psi_{i}\right\rangle=\left|n_{\mathbf{k} \lambda} ; a\right\rangle$ e $\left|\psi_{f}\right\rangle=\left|n_{\mathbf{k} \lambda}-1 ; b\right\rangle$, com $a$ e $b$ representando os estados de matéria. Portanto, a única contribuição não-nula para a probabilidade de transição será $d_{\mathbf{k} \lambda} \hat{E}_{\mathbf{k} \lambda}^{(+)}$, onde $d_{\mathbf{k} \lambda}=\mathbf{d} \cdot \mathbf{e}_{\mathbf{k} \lambda}$. A amplitude de transição se torna

$$
A_{i \rightarrow f} \sim\left\langle a\left|d_{\mathbf{k} \lambda}\right| b\right\rangle\left\langle f\left|\hat{E}_{\mathbf{k} \lambda}^{(+)}\right| i\right\rangle,
$$

onde $i$ e $f$ representam os estados inicial e final do campo. Portanto, a probabilidade de transição será

$$
P_{i \rightarrow f} \sim\left\langle i\left|\hat{E}_{\mathbf{k} \lambda}^{(-)}(x)\right| f\right\rangle\left\langle f\left|\hat{E}_{\mathbf{k} \lambda}^{(+)}(x)\right| i\right\rangle,
$$

que fornece a probabilidade de absorção do fóton em $\mathbf{r}$ no instante $t$. Como para cada estado inicial na base de Fock, existe apenas um final, podemos somar sobre todos os estados finais que fornecem contribuição nula.

$$
P_{i \rightarrow F} \sim \sum_{f}\left\langle i\left|\hat{E}^{(-)}(x)\right| f\right\rangle\left\langle f\left|\hat{E}^{(+)}(x)\right| i\right\rangle=\left\langle i\left|\hat{E}^{(-)}(x) \hat{E}^{(+)}(x)\right| i\right\rangle
$$

Até este ponto assumimos que o campo está em um estado inicial definido. Podemos considerar que este está em uma mistura estatística com peso $p_{i}$, portanto

$$
G^{(1)}(x)=\sum_{i} p_{i}\left\langle i\left|\hat{E}^{(-)}(x) \hat{E}^{(+)}(x)\right| i\right\rangle=\operatorname{Tr}\left[\hat{E}^{(-)}(x) \hat{E}^{(+)}(x) \rho\right]
$$

$\operatorname{com} x=(\mathbf{r}, t)$. Esta é a função de correlação de primeira ordem definida em ordem normal ${ }^{9}$. Ela mede a intensidade no detetor e é diagonal na base de estados coerentes. Para um detetor ideal, somente esta função de correlação seria necessária, pois todos os fótons de uma dada secção seriam absorvidos ao mesmo tempo e no mesmo ponto do espaço, liberando um elétron cada e estes não estariam correlacionados a nada além do fóton incidente. Para incorporar absorções em pontos do espaço e instantes diferentes que ocorrem em detetores não-ideais, a função de correlação pode ser generalizada como

$$
G^{(1)}\left(x, x^{\prime}\right)=\left\langle\hat{E}^{(-)}(x) \hat{E}^{(+)}\left(x^{\prime}\right)\right\rangle
$$

Para medidas feitas em mais de um detetor, estamos interessados em uma expressão

\footnotetext{
${ }^{9} \mathrm{~A}$ ordem normal é aquela na qual os operadores $\hat{a}$ aparecem sempre á direta de $\hat{a}^{\dagger}$.
} 
que forneça a probabilidade de que um fóton no modo $\mu$ seja absorvido na posição $\mathbf{r}$ e no tempo $t$ no detetor 1 condicionada à absorção de outro fóton no modo $\mu^{\prime}$ na posição $\mathbf{r}^{\prime} \mathrm{e}$ no tempo $t^{\prime}$ no detetor 2. A probabilidade de absorção de ambos os fótons é

$$
P_{i_{1}, i_{2} \rightarrow f_{1}, f_{2}} \sim\left\langle i_{1}\left|\hat{E}_{\mu}^{(-)}(x)\right| f_{1}\right\rangle\left\langle i_{1}\left|\hat{E}_{\mu}^{(+)}(x)\right| f_{1}\right\rangle\left\langle i_{2}\left|\hat{E}_{\mu^{\prime}}^{(-)}\left(x^{\prime}\right)\right| f_{2}\right\rangle\left\langle i_{2}\left|\hat{E}_{\mu^{\prime}}^{(+)}\left(x^{\prime}\right)\right| f_{2}\right\rangle .
$$

Faremos a soma sobre os estados finais e como os modos $\mu_{k}$ não afetam os estados $\left|i_{k^{\prime}}\right\rangle$, chegamos à expressão para a função de correlação de segunda ordem

$$
G_{\mu, \mu^{\prime}}^{(2)}\left(x, x^{\prime}\right)=\left\langle\hat{E}_{\mu}^{(-)}(x) \hat{E}_{\mu^{\prime}}^{(-)}\left(x^{\prime}\right) \hat{E}_{\mu^{\prime}}^{(+)}\left(x^{\prime}\right) \hat{E}_{\mu}^{(+)}(x)\right\rangle .
$$

Esta função de correlação será utilizada para calcular os espectros de ruído, que são as quantidades diretamente comparadas aos resultados experimentais. Ela também pode ser interpretada como uma medida das correlações de intensidade nos detetores, pois, ao tomar o limite clássico, ela converge para a correlação de intensidade

$$
G_{\mu, \mu^{\prime}}^{\text {Cláss }}\left(x ; x^{\prime}\right)=\left\langle I_{\mu}(x) I_{\mu^{\prime}}\left(x^{\prime}\right)\right\rangle \text {. }
$$

Todos os conceitos introduzidos neste capítulo serão utilizados no decorrer deste trabalho tanto no desenvolvimento da teoria quanto na descrição do experimento. No próximo capítulo trataremos a interação do campo eletromagnético com o átomo de dois níveis. 


\section{$3 \quad$ Sistema de 2 Niveis}

Neste capítulo trataremos do problema de um átomo de dois níveis interagindo com um campo quantizado. O sistema de dois níveis é o sistema mais simples para descrever átomos interagindo com a radiação eletromagnética e é equivalente, em vários aspectos, a um férmion de spin $1 / 2$ [46, 47]. Alguns resultados diretamente obtidos para este tipo de sistema serão utilizados no decorrer do trabalho, inclusive na descrição dos métodos experimentais, pois diversas técnicas de controle utilizadas são baseadas em modelos que consideram o átomo como um sistema de dois níveis. O principal objetivo deste capítulo é servir de laboratório para realizar as contas e introduzir os conceitos físicos em um contexto mais simples. Os resultados demonstrados, assim como os métodos utilizados, serão generalizados nos dois próximos capítulos, nos quais o fenômeno da EIT será tratado de forma direta. A abordagem tratada aqui segue a mesma linha de [48, 49, 47].

\subsection{Formulação do Problema}

Fisicamente, o sistema é formado por três subsistemas: o átomo, o modo laser, que possui número médio de fótons elevado e cuja média fornece o campo clássico, e os modos do campo que estão no vácuo, que serão tratados como um reservatório. A figura 3.1 mostra a interação entre os subsistemas. O campo laser que incide sobre os átomos não interage com os modos que estão no vácuo, diferentemente do que ocorre em uma cavidade, em que esta interação é mediada pelo espelho de acoplamento. Como o operador campo elétrico é o mesmo independentemente de quantos modos estão populados e como estes modos não interagem diretamente, trataremos o modo laser e os modos do vácuo como apenas um subsitema por enquanto.

O hamiltoniano do sistema é formado por uma parte dependente do campo, uma do átomo e um termo de interação que depende de variáveis de ambos subsistemas,

$$
H=H_{0}+H_{f}+V
$$




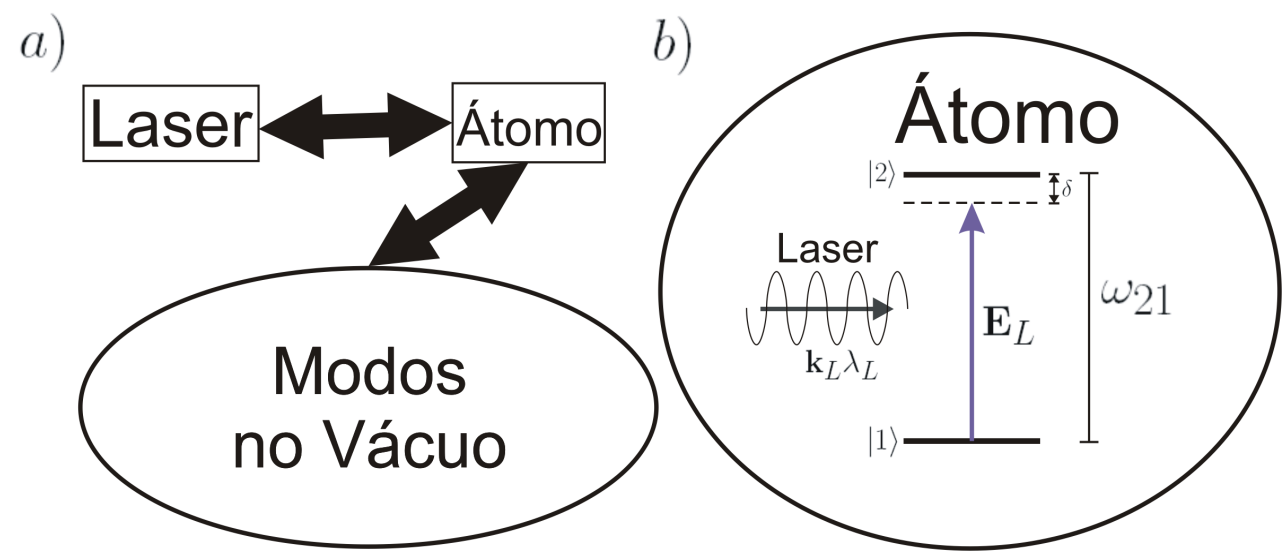

Figura 3.1: a) Figura esquemática da interação entre os subsistemas b) Interação entre o átomo e o modo laser

sendo que cada contribuição está definida no formalismo de Schrödinger. O hamiltoniano atômico, na base dos seus autoestados, é

$$
H_{0}=\sum_{j=1}^{2} \hbar \omega_{j} \hat{\sigma}_{j j}
$$

onde $|2\rangle$ é o estado excitado, $|1\rangle$ é o fundamental, como visto na figura 3.1. Os operadores atômicos são definidos como $\hat{\sigma}_{i j}=|i\rangle\langle j|$, com $i, j=1,2$. Entre eles estão tanto os projetores quanto os operadores de subida e descida. Suas relações de comutação são

$$
\left[\hat{\sigma}_{i j}, \hat{\sigma}_{l m}\right]=\delta_{j l} \hat{\sigma}_{i m}-\delta_{i m} \hat{\sigma}_{l j}
$$

O hamiltoniano do campo no formalismo de Schrödinger será o hamiltoniano de campo livre,

$$
H_{f}=\sum_{\mathbf{k}, \lambda} \hbar \omega_{k}\left(\hat{a}_{\mathbf{k}, \lambda}^{\dagger} \hat{a}_{\mathbf{k}, \lambda}+\frac{1}{2}\right) .
$$

Como discutido no Apêndice A, o hamiltoniano de interação será o hamiltoniano de dipolo elétrico,

$$
V=-\hat{\mathbf{d}} \cdot \hat{\mathbf{E}}
$$

onde $\hat{\mathbf{d}}=-e \hat{\mathbf{R}}$ é o operador de dipolo atômico. Reescreveremos a componente de frequência positiva (2.66) no formalismo de Schrödinger e na aproximação de longo compimento de onda, discutida no Apêndice A, como

$$
\hat{\mathbf{E}}^{(+)}=i \sum_{\mathbf{k} \lambda} \sqrt{\frac{2 \pi \hbar \omega}{V}} \hat{a}_{\mathbf{k} \lambda} \mathbf{e}_{\mathbf{k} \lambda},
$$


$\hat{\mathbf{E}}^{(-)}=\left[\hat{\mathbf{E}}^{(+)}\right]^{\dagger}$ e $\left[\hat{a}_{\mathbf{k} \lambda}, \hat{\sigma}_{i j}\right]=0$. O operador de dipólo pode ser expandido na base $|j\rangle$ como

$$
\hat{\mathbf{d}}=\sum_{i j} \mathbf{d}_{i j} \hat{\sigma}_{i j}
$$

onde $\mathbf{d}_{i j}=\langle i|\mathbf{d}| j\rangle$. Como $\hat{\mathbf{d}}$ é ímpar por inversão espacial, o elemento de matriz $\mathbf{d}_{i j}$ será não-nulo se os estados $|i\rangle$ e $|j\rangle$ tiverem paridade definida e oposta [29, 26, 27]. Como os estados que usamos são níveis hiperfinos, que possuem paridade bem definida e associada à paridade do momento angular total, então $\langle i|\mathbf{d}| i\rangle=0$, com $i=1,2$, portanto

$$
\hat{\mathbf{d}}=\mathbf{d}_{12} \hat{\sigma}_{12}+\mathbf{d}_{21} \hat{\sigma}_{21}
$$

e a expressão (3.5) fica

$$
V=-\left(\mathbf{d}_{12} \hat{\sigma}_{12}+\mathbf{d}_{21} \hat{\sigma}_{21}\right) \cdot\left(\hat{\mathbf{E}}^{(+)}+\hat{\mathbf{E}}^{(-)}\right)
$$

A solução exata do problema usando este hamiltoniano de interação é difícil de ser obtida mesmo utilizando campos clássicos. Uma primeira forma de tratar este problema é fazendo perturbações no hamiltoniano livre $[50,47,26]$. As soluções perturbativas em primeira ordem para os estados, utilizando campos clássicos, estão em função de dois termos: um cujo denominador é $\omega_{0}-\omega_{L}$ e está multiplicado por um fator $\exp \left[i\left(\omega_{0}-\omega_{L}\right) t\right]$, e outro cujo demoninador é $\omega_{0}+\omega_{L}$ e está multiplicado por $\exp \left[i\left(\omega_{0}+\omega_{L}\right) t\right]$, tal que $\omega_{0}$ e $\omega_{L}$ sejam, respectivamente, as frequêcias de Bohr da transição atômica e do campo incidente.

Para o regime de campo quase ressonante, $\omega_{0} \approx \omega_{L}$, o primeiro termo fica muito maior que o segundo e oscila mais lentemente também. Nestas condições, podemos desprezar o segundo termo da solução. Esta solução é a mesma que seria obtida se, já no hamiltoniano de interação, os operadores que dão origem à solução de alta frequência, chamados termos contragirantes, fossem desprezados. Esta aproximação em que ingnoramos os termos contragirantes é chama de Aproximação de Onda Girante (no inglês RWA) e será utilizada em todo este trabalho.

No caso do campo quantizado, podemos interpretar os termos contragirantes como processos que não conservam energia em primeira ordem de perturbação. Estes termos são $\hat{\mathbf{E}}^{(+)} \hat{\sigma}_{12}$, que representa o processo em que o átomo vai do estado excitado para o fundamental e um fóton é absorvido, e $\hat{\mathbf{E}}^{(-)} \hat{\sigma}_{21}$, que representa o processo inverso, o átomo vai do estado fundamental para o excitado e um fóton é emitido pelo átomo. Neste contexto, a RWA consiste em ignorar estes dois termos no hamiltoniano de interação,

$$
V_{R W A}=-\mathbf{d}_{12} \cdot \hat{\mathbf{E}}^{(-)} \hat{\sigma}_{12}-\mathbf{d}_{21} \cdot \hat{\mathbf{E}}^{(+)} \hat{\sigma}_{21}
$$


Note que ainda há a necessidade de campo ser quase ressonante, pois, as contribuições de primeira ordem devido as termos contragirante somente se tornam pequenas apenas quando $\omega_{0} \approx \omega_{L}$. Uma das vantagens da RWA sobre as soluções perturbativas é que estamos susbtituindo um hamiltoniano hermitiano por um hamiltoniano efetivo também hermitiano e mais simples. Mais detalhes podem ser vistos em [46, 47, 34, 51].

Apesar de o tratamento teórico ser feito para a interação de apenas um átomo com o campo, o nosso experimento não reproduz diretamente esta teoria, pois é feito em uma célula de vapor com comprimento finito. Um tratamento mais geral, que leva em conta a propagação do campo quantizado, é feito por meio das equações de Maxwell-HeisenbergLangevin [13, 21] e, para o campo clássico, pelas equações de Maxwell-Bloch [40]. Em nosso caso, como a densidade de átomos é muito baixa, o nosso tratamento é validado, pois átomos em posições diferente da amostra irão interagir com campos de mesma amplitude aproximadamente, mas com fases relativas diferentes dadas pela propagação ${ }^{1}$. O que significa que se um átomo em $z=0$ interage com o campo laser $\hat{\mathbf{E}}_{L}^{(+)}(t)$, outro átomo em $z=z_{0}$ irá interagir com $\hat{\mathbf{E}}_{L}^{(+)}\left(z_{0}, t\right)=\hat{\mathbf{E}}_{L}^{(+)}(t) e^{i k z_{0}}$, considerando que o laser se propaga na direção $z$. Note que ainda assim a aproximação de longo comprimento de onda é válida.

\subsection{Equações de Heisenberg-Langevin}

O hamiltoniano (3.8) considera a interação do átomo com todos os modos do campo. No caso em que temos um laser ideal, apenas um modo do campo está populado e contém muitos fótons. Para este tipo de sistema, um modelo mais simples, chamado modelo de Jaynes-Cunnings, é bastante utilizado [50, 47, 46], no qual a interação do átomo com os modos não populados do campo é desprezada. Isto, em princípio, não pode ser feito, pois o estado do sistema físico não é mais definido pelo campo elétrico como no Eletromagnatismo Clássico. Mesmo porque o estado do sistema agora é representado por um vetor no espaço de Hilbert e o campo elétrico é um operador que atua neste espaço. Desta forma, mesmo que o campo que interaja com o sistema atômico seja monomodo, ainda pode haver acoplamento com modos não populados que darão origem a fenômenos físicos importantes, como a emissão espontânea, que será tratada mais adiante. Tendo isto em vista, passaremos ao formalismo de Heisenberg utilizando todos os modos do operador

\footnotetext{
${ }^{1}$ Nesta aproximação, em que o campo incidente praticamente não é perturbado pela presença dos átomos, o meio é chamado de oticamente fino.
} 
campo elétrico em (3.6),

$$
\begin{array}{r}
\hat{a}_{\mathbf{k} \lambda}(t)=U^{\dagger}(t) \hat{a}_{\mathbf{k} \lambda} U(t), \\
\hat{\sigma}_{i j}(t)=U^{\dagger}(t) \hat{\sigma}_{i j} U(t),
\end{array}
$$

onde $U(t)=\exp \left[-i\left(H_{0}+H_{f}+V_{R W A}\right) t / \hbar\right]$, tal que $\left[\hat{a}_{\mathbf{k} \lambda}(t), \hat{\sigma}_{i j}(t)\right]=0$ e $\hat{a}_{\mathbf{k} \lambda}(t)$ não será o mesmo que em (2.62), pois, além do campo livre, há a contribuição devido à interação com o átomo. As equações de Heisenberg para $\hat{a}_{\mathbf{k} \lambda}(t)$ e $\hat{\sigma}_{i j}(t)$ serão

$$
\begin{aligned}
\frac{d \hat{a}_{\mathbf{k} \lambda}(t)}{d t}= & -i \omega \hat{a}_{\mathbf{k} \lambda}(t)+\sqrt{\frac{2 \pi \omega}{V \hbar}}\left(\mathbf{e}_{\mathbf{k} \lambda}^{*} \cdot \mathbf{d}_{12}\right) \hat{\sigma}_{12}(t) \\
\frac{d \hat{\sigma}_{i j}(t)}{d t}= & i \omega_{i j} \hat{\sigma}_{i j}(t)+i \frac{\mathbf{d}_{12} \cdot \hat{\mathbf{E}}^{(-)}(t)}{\hbar}\left[\delta_{j 1} \hat{\sigma}_{i 2}(t)-\delta_{i 2} \hat{\sigma}_{1 j}(t)\right] \\
& +i \frac{\mathbf{d}_{21} \cdot \hat{\mathbf{E}}^{(+)}(t)}{\hbar}\left[\delta_{j 2} \hat{\sigma}_{i 1}(t)-\delta_{i 1} \hat{\sigma}_{2 j}(t)\right]
\end{aligned}
$$

com $\omega_{i j}=\omega_{i}-\omega_{j}$. Como $\hat{\mathbf{E}}^{(-)}(t)$ e $\hat{\mathbf{E}}^{(+)}(t)$ são funções de todos $\hat{a}_{\mathbf{k} \lambda}^{\dagger}(t)$ e $\hat{a}_{\mathbf{k} \lambda}(t)$, as equações (3.10) e (3.11) formam um conjunto infinito de equações diferenciais não-lineares e acopladas. Na sua forma mais geral, esse sistema é impossível de ser resolvido diretamente. Mas, devido ao estado do campo estar restrito a apenas um modo populado e os outros formando um reservatório, consiguiremos resolver o problema dentro de certas aproximações. Em primeiro lugar, pelo próprio uso da RWA, o campo deve ser quase ressonante. Mais que isso, supomos que ele seja monomodo dentro da precisão em que podemos resolver a linha atômica. Desta forma, o operador densidade do sistema é

$$
\rho_{T}=\rho_{A} \otimes \rho_{L} \otimes \rho_{0}
$$

onde $\rho_{A}$ representa o sistema atômico, $\rho_{L}$ o modo da fonte laser e $\rho_{0}=|0\rangle\langle 0|$ é o produto tensorial dos modos do reservatório. Multiplicando por $e^{i \omega t}$ nos dois lados de (3.10) e integrando obtemos

$$
\hat{a}_{\mathbf{k} \lambda}(t)=e^{-i \omega t} \hat{a}_{\mathbf{k} \lambda}+\sqrt{\frac{2 \pi \omega}{V \hbar}}\left(\mathbf{e}_{\mathbf{k} \lambda}^{*} \cdot \mathbf{d}_{12}\right) \int_{0}^{t} e^{-i \omega\left(t-t^{\prime}\right)} \hat{\sigma}_{12}\left(t^{\prime}\right) d t^{\prime} .
$$

O primeiro termo é referente ao campo livre e o segundo é o efeito da interação com o átomo. Este depende tanto do operador atômico quanto da direção do modo $\mathbf{k} \lambda$. Então, 


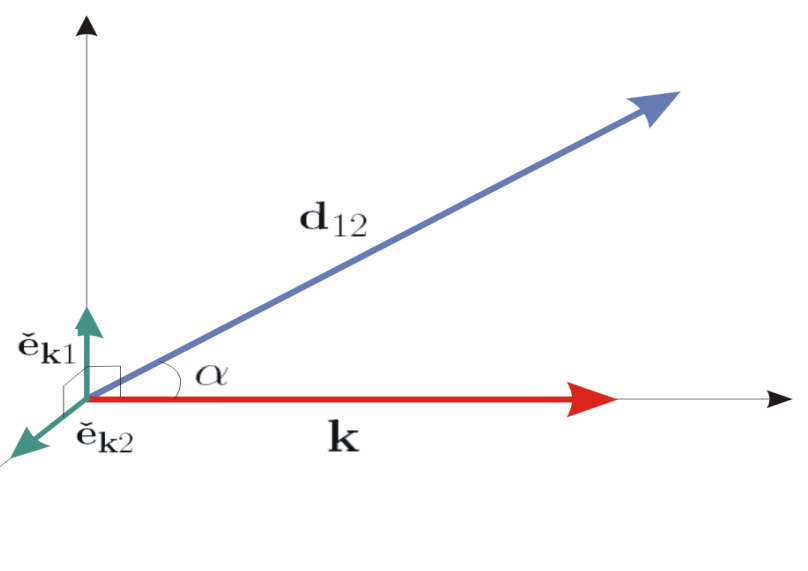

Figura 3.2: Sistema de Coordenadas para cada $\mathbf{k}$

susbtituindo em (3.6) podemos escrever $\hat{\mathbf{E}}^{(+)}(t)=\hat{\mathbf{E}}_{l}^{(+)}(t)+\hat{\mathbf{E}}_{p}^{(+)}(t)$, onde

$$
\begin{aligned}
& \hat{\mathbf{E}}_{l}^{(+)}(t)=i \sum_{\mathbf{k} \lambda} \sqrt{\frac{2 \pi \hbar \omega}{V}} \hat{a}_{\mathbf{k} \lambda} \mathbf{e}_{\mathbf{k} \lambda} e^{-i \omega t}, \\
& \hat{\mathbf{E}}_{p}^{(+)}(t)=i \sum_{\mathbf{k} \lambda} \frac{2 \pi \omega}{V} \mathbf{v}_{\mathbf{k} \lambda} \int_{0}^{t} e^{-i \omega\left(t-t^{\prime}\right)} \hat{\sigma}_{12}\left(t^{\prime}\right) d t^{\prime},
\end{aligned}
$$

com $\mathbf{v}_{\mathbf{k} \lambda}=\left(\mathbf{e}_{\mathbf{k} \lambda}^{*} \cdot \mathbf{d}_{12}\right) \mathbf{e}_{\mathbf{k} \lambda}$. Como pode ser visto, $\hat{\mathbf{E}}_{l}(t)$ é o operador para o campo livre obtido em (2.64), para $\mathbf{r}=0$, e $\hat{\mathbf{E}}_{p}(t)$ será chamado de campo de polarização. O nome é justificado, pois este operador é proporcional a uma projeção de uma componente do operador de dipolo atômico, além de a expressão para o campo total sugerir este nome. Como esperado, ele depende dos operadores atômicos em todos os instantes $t^{\prime}$, tal que $0 \leq t^{\prime} \leq t$, sendo que a exponencial faz o papel da função resposta.

Como estamos tratando de um campo propagante, podemos tomar o limite de espectro contínuo e transformar a soma em uma integral via (2.68). Também escolheremos a base de polarização linear, $\mathbf{e}_{\mathbf{k} \lambda}=\mathbf{e}_{\mathbf{k} \lambda}^{*}[29,27]$. Como mostrado na figura 3.2, podemos escolher a base no espaço transverso $\left(\mathbf{e}_{\mathbf{k} 1}, \mathbf{e}_{\mathbf{k} 2}\right)$ tal que $\mathbf{d}_{12} \perp \mathbf{e}_{\mathbf{k} 2}$, e analisando a direção e módulo de $\mathbf{v}_{\mathbf{k} \lambda}$, chegamos ao seguinte resultado, $\mathbf{v}_{\mathbf{k} 1}=\left(\mathbf{e}_{\mathbf{k} 1} \cdot \mathbf{d}_{12}\right) \mathbf{e}_{\mathbf{k} 1}=-\left(\mathbf{d}_{12} \times \mathbf{k}_{\mathbf{u}}\right) \times \mathbf{k}_{u}$ e $\mathbf{v}_{\mathbf{k} 2}=0$, onde $\mathbf{k}_{u}=\mathbf{k} / k$. Já passando para coordenadas esféricas, usando $\omega=k c$ e $d^{3} k=\left(\omega^{2} / c^{3}\right) \sin \theta d \omega d \theta d \phi$, a expressão (3.15) fica

$$
\hat{\mathbf{E}}_{p}^{(+)}(t)=-\frac{i}{4 \pi^{2} c^{3}} \int_{V} d \omega d \theta d \phi \sin \theta \omega^{3}\left[\left(\mathbf{d}_{12} \times \mathbf{k}_{\mathbf{u}}\right) \times \mathbf{k}_{\mathbf{u}}\right] \int_{0}^{t} e^{-i \omega\left(t-t^{\prime}\right)} \hat{\sigma}_{12}\left(t^{\prime}\right) d t^{\prime} .
$$

Para realizar a integração, escolhemos $\mathbf{d}_{12}$ paralelo ao vetor $\mathbf{k}_{z}$,

$$
\mathbf{k}_{u}=\left(\mathbf{d}_{12}\right)_{u} \cos \theta+\mathbf{k}_{y} \sin \theta \sin \phi+\mathbf{k}_{x} \sin \theta \cos \phi
$$


onde $\left(\mathbf{d}_{12}\right)_{u}$ é o vetor unitário da direção de $\mathbf{d}_{12}$ e $\mathbf{k}_{x, y, z}$ são os vetores unitários da base cartesiana para cada k. Realizando os produtos vetoriais e integrando em $d \phi$, chegamos a

$$
\hat{\mathbf{E}}_{p}^{(+)}(t)=\frac{i \mathbf{d}_{12}}{2 \pi c^{3}} \int_{0}^{\infty} \int_{0}^{\pi} d \omega d \theta \sin ^{3} \theta \omega^{3} \int_{0}^{t} e^{-i \omega\left(t-t^{\prime}\right)} \hat{\sigma}_{12}\left(t^{\prime}\right) d t^{\prime} .
$$

A integração em $\theta$ é direta e seu resultado é $4 / 3$. Pelo fato do campo ser quase ressonante, faremos a mudança de variável, $\omega=\omega_{0}+\Omega$, com $\Omega \ll \omega_{0}$ e a integração será feita somente sobre $|\Omega| \approx 0$. Como na expressão acima a exponencial varia muito mais rapidamente que a potência cúbica, podemos aproximar $\omega^{3} \approx \omega_{0}^{3}$ e tirá-la da integral. Como o limite inferior em $d \Omega$ é $-\omega_{0}$, podemos estendê-lo até $-\infty$, portanto,

$$
\hat{\mathbf{E}}_{p}^{(+)}(t)=\frac{2 i \mathbf{d}_{12} \omega_{0}^{3}}{3 \pi c^{3}} \int_{-\infty}^{\infty} d \Omega \int_{0}^{\infty} e^{-i \omega_{0}\left(t-t^{\prime}\right)} e^{-i \Omega\left(t-t^{\prime}\right)} \hat{\sigma}_{12}\left(t^{\prime}\right) \theta\left(t-t^{\prime}\right) d t^{\prime}
$$

onde $\theta\left(t-t^{\prime}\right)$ é a função degrau, definida como

$$
\theta(x)=\left\{\begin{array}{cc}
0, & \mathrm{x}>0 \\
1, & \mathrm{x}<0 \\
\frac{1}{2}, & \mathrm{x}=0
\end{array}\right.
$$

Realizando as integrais em $d \Omega$ e $d t$, obtemos a contribuição da polarização na posição do átomo,

$$
\hat{\mathbf{E}}_{p}^{(+)}(t)=\frac{2 i \mathbf{d}_{12} \omega_{0}^{3}}{3 c^{3}} \hat{\sigma}_{12}(t)
$$

Neste caso, a função resposta é uma delta de Dirac, que surge ao somarmos sobre os todos os modos do campo. Esse tratamento é possível, pois o campo possui infinitos graus de liberdade em comparação com o átomo. O fato de influências do átomo sobre o campo e, consequentemente, do campo sobre o átomo não incluírem tempos anteriores inplica em um processo markoviano $[52,30,53,54]$. Pelo fato de possuírem muitos graus de liberdade, os modos no vácuo atuam como um reservatório que perde a memória de tempos anteriores. Este, à medida que troca energia com o sistema por meio do hamiltoniano (3.8), origina termos dissipativos. Neste ponto, se substituímos (3.18) em (3.11) obteremos um sistema de equações diferenciais de primeira ordem para $\hat{\sigma}_{i j}$. 
Para mais fácil visualização, abriremos a equação (3.11) em componentes,

$$
\begin{aligned}
& \dot{\hat{\sigma}}_{11}=i \frac{\mathbf{d}_{12} \cdot \hat{\mathbf{E}}^{(-)}(t)}{\hbar} \hat{\sigma}_{12}(t)-i \frac{\mathbf{d}_{21} \cdot \hat{\mathbf{E}}^{(+)}(t)}{\hbar} \hat{\sigma}_{21}(t), \\
& \dot{\hat{\sigma}}_{22}=-i \frac{\mathbf{d}_{12} \cdot \hat{\mathbf{E}}^{(-)}(t)}{\hbar} \hat{\sigma}_{12}(t)+i \frac{\mathbf{d}_{21} \cdot \hat{\mathbf{E}}^{(+)}(t)}{\hbar} \hat{\sigma}_{21}(t), \\
& \dot{\hat{\sigma}}_{12}=-i \omega_{0} \hat{\sigma}_{12}-i \frac{\mathbf{d}_{21} \cdot \hat{\mathbf{E}}^{(+)}(t)}{\hbar}\left[\hat{\sigma}_{22}(t)-\hat{\sigma}_{11}(t)\right], \\
& \dot{\hat{\sigma}}_{21}=\left[\dot{\hat{\sigma}}_{12}\right]^{\dagger},
\end{aligned}
$$

onde $\omega_{0}=\omega_{2}-\omega_{1}$ é a frequência de Bohr da transição. Para substituir (3.18) nas equações (3.19)-(3.22), certo cuidado deve ser tomado, pois apesar de $\left[\hat{\mathbf{E}}^{( \pm)}, \hat{\sigma}_{i j}(t)\right]=0$, $\left[\hat{\mathbf{E}}_{p}^{( \pm)}(t), \hat{\sigma}_{i j}(t)\right] \neq 0$ e $\left[\hat{\mathbf{E}}_{l}^{( \pm)}(t), \hat{\sigma}_{i j}(t)\right] \neq 0$. Sendo assim, iremos escolher uma ordem na qual fazer essa substituição e que deverá ser seguida por todo o cálculo. Escolheremos a ordem normal, na qual os operadores serão ordenados da seguinte forma,

$$
\hat{a}_{\mathbf{k} \lambda}^{\dagger}, \hat{\sigma}_{21}(t), \hat{\sigma}_{j j}(t), \hat{\sigma}_{12}(t), \hat{a}_{\mathbf{k} \lambda}
$$

$\operatorname{com} j=1,2$.

Ao fazer a substituição, o sistema (3.19)-(3.22) se resume ao conjunto de equações de Heisenberg-Langevin, que representam um análogo quântico das equações de Langevin $[47,54,30,52]$,

$$
\begin{aligned}
& \dot{\hat{\sigma}}_{11}=i \hat{g}_{L}^{\dagger} \hat{\sigma}_{12}(t) e^{i \omega_{L} t}+-i \hat{\sigma}_{21}(t) \hat{g}_{L} e^{-i \omega_{L} t}+\Gamma \hat{\sigma}_{22}(t)+\hat{F}_{11}(t), \\
& \dot{\hat{\sigma}}_{22}=-i \hat{g}_{L}^{\dagger} \hat{\sigma}_{12}(t) e^{i \omega_{L} t}+i \hat{\sigma}_{21}(t) \hat{g}_{L} e^{-i \omega_{L} t}-\Gamma \hat{\sigma}_{22}(t)+\hat{F}_{22}(t), \\
& \dot{\hat{\sigma}}_{12}=-i \omega_{0} \hat{\sigma}_{12}(t)-\frac{\Gamma}{2} \hat{\sigma}_{12}(t)-i\left[\hat{\sigma}_{22}(t)-\hat{\sigma}_{11}(t)\right] \hat{g}_{L} e^{-i \omega_{L} t}+\hat{F}_{12}(t), \\
& \dot{\hat{\sigma}}_{21}=\left[\dot{\hat{\sigma}}_{12}\right]^{\dagger}
\end{aligned}
$$

De modo que $\hat{g}_{\mathbf{k} \lambda}$ e $\Gamma$ são, respectivamente, a frequência de Rabi por fóton no modo $\mathbf{k} \lambda$ e a taxa de emissão espontânea[40, 46, 48, 47], que são definidas como

$$
\begin{aligned}
\Gamma & =\frac{4}{3} \frac{\left|\mathbf{d}_{12}\right|^{2} \omega_{0}^{3}}{\hbar c^{3}} \\
\hat{g}_{\mathbf{k} \lambda} & =i \sqrt{\frac{2 \pi \omega}{\hbar V}}\left(\mathbf{e}_{\mathbf{k} \lambda} \cdot \mathbf{d}_{21}\right) \hat{a}_{\mathbf{k} \lambda} .
\end{aligned}
$$

e $\hat{g}_{L} \equiv \hat{g}_{\mathbf{k}_{L} \lambda_{L}}$ é uma notação simplificada para o modo laser. As forças quânticas de 
Langevin, $\hat{F}_{i j}$, surgem naturalmente e são dadas por

$$
\begin{aligned}
& \hat{F}_{11}(t)=\sum_{\mathbf{k} \neq \mathbf{k}_{L} \lambda \neq \lambda_{L}}+i \hat{g}_{\mathbf{k} \lambda}^{\dagger} e^{i \omega t} \hat{\sigma}_{12}(t)-i e^{-i \omega t} \hat{\sigma}_{21}(t) \hat{g}_{\mathbf{k} \lambda} \\
& \hat{F}_{22}(t)=\sum_{\mathbf{k} \neq \mathbf{k}_{L} \lambda \neq \lambda_{L}}-i \hat{g}_{\mathbf{k} \lambda}^{\dagger} e^{i \omega t} \hat{\sigma}_{12}(t)+i e^{-i \omega t} \hat{\sigma}_{21}(t) \hat{g}_{\mathbf{k} \lambda}, \\
& \hat{F}_{12}(t)=\sum_{\mathbf{k} \neq \mathbf{k}_{L} \lambda \neq \lambda_{L}}-i e^{-i \omega t}\left[\hat{\sigma}_{22}(t)-\hat{\sigma}_{11}(t)\right] \hat{g}_{\mathbf{k} \lambda}, \\
& \hat{F}_{21}(t)=\left[\hat{F}_{12}(t)\right]^{\dagger}
\end{aligned}
$$

Para mais fácil manipulação, definimos o vetor $\hat{\mathbf{f}}(t)=\left(\hat{F}_{11}(t), \hat{F}_{22}(t), \hat{F}_{12}(t), \hat{F}_{21}(t)\right)^{T}$. Ao efetuármos a média no operador densidade (3.12), mostramos que

$$
\left\langle\hat{f}_{i}(t)\right\rangle=0
$$

A correlação temporal entre $\hat{f}_{i}(t)$ e $\hat{f}_{j}^{\dagger}\left(t^{\prime}\right)$ pode ser calculada usando (3.28)-(3.31). Calcularemos uma delas como exemplo,

$$
\left\langle\hat{f}_{1}(t) \hat{f}_{1}\left(t^{\prime}\right)\right\rangle=\sum_{\mathbf{k} \neq \mathbf{k}_{L} \lambda \neq \lambda_{L}} \sum_{\mathbf{k}^{\prime} \neq \mathbf{k}_{L} \lambda^{\prime} \neq \lambda_{L}} \operatorname{Tr}\left[\hat{\sigma}_{21} U(t) \hat{g}_{\mathbf{k} \lambda} \hat{g}_{\mathbf{k}^{\prime} \lambda^{\prime}}^{\dagger} e^{-i \omega t+i \omega^{\prime} t^{\prime}} \hat{\sigma}_{12}\left(t^{\prime}\right) U^{\dagger}(t) \rho_{T}(t)\right]
$$

Como os modos no vácuo irão atuar como um reservatório sem memória de tempos anteriores, o operador densidade do reservatório não evolui no tempo. Tendo isso em vista, assumimos que

$$
\rho_{T}(t)=\rho_{A+L}(t) \otimes \rho_{0}
$$

Nesta aproximação, desprezamos também as correlações do reservatório com o subsistema átomo+laser. Por já se tratar do cálculo de $\left\langle\hat{f}_{i}(t) \hat{f}_{j}\left(t^{\prime}\right)\right\rangle$, desprezar as correlações neste ponto equivale a parar o problema em segunda ordem. Como $\rho_{0}=U(t) \rho_{0} U^{\dagger}(t)^{2}$, então $\left[U(t), \rho_{0}\right]=0$. Sendo assim,

$$
\left\langle\hat{f}_{1}(t) \hat{f}_{1}\left(t^{\prime}\right)\right\rangle=\frac{2 \pi}{\hbar V} \sum_{\mathbf{k} \neq \mathbf{k}_{L} \lambda \neq \lambda_{L}} \omega\left(\mathbf{e}_{\mathbf{k} \lambda}^{*} \cdot \mathbf{d}_{12}\right) \mathbf{e}_{\mathbf{k} \lambda} \cdot \mathbf{d}_{21} \operatorname{Tr}\left[\hat{\sigma}_{21} U(t) e^{-i \omega\left(t-t^{\prime}\right)} \hat{\sigma}_{12}\left(t^{\prime}\right) U^{\dagger}(t) \rho_{T}(t)\right] .
$$

pois $\hat{g}_{\mathbf{k} \lambda} \hat{g}_{\mathbf{k}^{\prime} \lambda^{\prime}}^{\dagger} \rho_{0}=\frac{2 \pi \omega}{\hbar V}\left|\mathbf{e}_{\mathbf{k} \lambda} \cdot \mathbf{d}_{21}\right|^{2} \delta_{\mathbf{k}, \mathbf{k}^{\prime}} \delta_{\lambda, \lambda^{\prime}} \rho_{0}$. Ao passar para o limite contínuo obteremos

$$
\left\langle\hat{f}_{1}(t) \hat{f}_{1}\left(t^{\prime}\right)\right\rangle=\frac{-\operatorname{Tr}\left[\hat{\sigma}_{21} U(t) \hat{\sigma}_{12}\left(t^{\prime}\right) U^{\dagger}(t) \rho_{T}(t)\right]}{4 \pi^{2} c^{3} \hbar} \int_{V} d \omega d \theta d \phi \sin \theta \omega^{3}\left[\left(\mathbf{d}_{12} \times \mathbf{k}_{\mathbf{u}}\right) \times \mathbf{k}_{\mathbf{u}}\right] e^{-i \omega\left(t-t^{\prime}\right)} \cdot \mathbf{d}_{21},
$$

cuja integral é a mesma de (3.16) a menos da integração no tempo, que após resolvida fica $\left\langle\hat{f}_{1}(t) \hat{f}_{1}\left(t^{\prime}\right)\right\rangle=\Gamma \operatorname{Tr}\left[\hat{\sigma}_{21} U(t) \hat{\sigma}_{12}\left(t^{\prime}\right) U^{\dagger}(t) \rho_{T}(t)\right] \delta\left(t-t^{\prime}\right) . \operatorname{Como} \operatorname{Tr}\left[\hat{\sigma}_{21} U(t) \hat{\sigma}_{12}\left(t^{\prime}\right) U^{\dagger}(t) \rho_{T}(t)\right]=$

\footnotetext{
${ }^{2}$ Pelo fato de $U(t)$ possuir dimensão maior que $\rho_{0}$, o significado preciso desta expressão é $\mathbb{I} \times \mathbb{I} \rho_{0} U(t) \mathbb{I} \times$ $\mathbb{I} \rho_{0} U^{\dagger}(t)$.
} 
$\operatorname{Tr}\left[\hat{\sigma}_{22}(t) \rho_{T}\right]$, chegamos á expressão

$$
\left\langle\hat{f}_{1}(t) \hat{f}_{1}\left(t^{\prime}\right)\right\rangle=-\Gamma\left\langle\hat{\sigma}_{22}(t)\right\rangle \delta\left(t-t^{\prime}\right)
$$

Como no caso das forças de Langevin estocásticas, as forças quânticas de Langevin são correlacionadas apenas no mesmo tempo, o que era de se esperar por se tratar de um sistema markoviano. Um ruído que se comporta como em (3.33) é chamado de ruído branco e ocorre no processo estocástico de Wiener [52, 53]. Ao fazer o mesmo cálculo para todas as correlções possíveis chegamos a

$$
\left\langle\hat{f}_{i}(t) \hat{f}_{j}^{\dagger}\left(t^{\prime}\right)\right\rangle=D_{i j} \delta\left(t-t^{\prime}\right)
$$

com $D_{i j}$ sendo as componentes da matriz de difusão ${ }^{3}$

$$
\mathbf{D}=\left(\begin{array}{cccc}
-\Gamma\left\langle\hat{\sigma}_{22}\right\rangle & 0 & 0 & 0 \\
0 & \Gamma\left\langle\hat{\sigma}_{22}\right\rangle & 0 & \Gamma\left\langle\hat{\sigma}_{21}\right\rangle \\
0 & \Gamma\left\langle\hat{\sigma}_{12}\right\rangle & 0 & \Gamma\left(\left\langle\hat{\sigma}_{11}\right\rangle+\left\langle\hat{\sigma}_{22}\right\rangle\right) \\
0 & 0 & 0 & 0
\end{array}\right)
$$

Pelo fato de as variáveis do reservatório e do modo laser serem descorrelacionadas, para quaisquer funções das variáveis do laser $g_{i}\left(\hat{a}_{L}, \hat{a}_{L}^{\dagger}\right)$, com $i=1,2$, obteremos

$$
\left\langle g_{1}\left(\hat{a}_{L}, \hat{a}_{L}^{\dagger}\right) \hat{f}_{i}(t) g_{2}\left(\hat{a}_{L}, \hat{a}_{L}^{\dagger}\right)\right\rangle=0
$$

Ao passar para o domínio da frequência, as correlações de as forças de Langevin serão

$$
\left\langle\hat{f}_{i}(\omega) \hat{f}_{j}^{\dagger}\left(\omega^{\prime}\right)\right\rangle=D_{i j} \delta\left(\omega+\omega^{\prime}\right)
$$

Utilizaremos esta matriz para cálcular o espectro de ruído, pois ela fornecerá a contribuição dos modos no vácuo. Considerações sobre $\left\langle\hat{\sigma}_{i j}\right\rangle$, assim como a derivação da equação satisfeita por eles, serão feitas na próxima seção.

Este sistema, entretanto, possui um vínculo, pois, como $\hat{\sigma}_{11}+\hat{\sigma}_{22}=1, \dot{\hat{\sigma}}_{11}=-\dot{\hat{\sigma}}_{22}$, o que pode ser visto em (3.23) e (3.24). Sendo assim, o sistema (3.23)-(3.26) pode ser reduzido em uma equação. E faremos a seguinte mudança de variáveis para tirar a dependência temporal contida na exponencial, que equivale eliminar a dependência rápida

\footnotetext{
${ }^{3}$ As componentes da matriz de difusão podem ser calculadas utilizando as relações generalizadas de Einstein[47].
} 
de $\hat{\sigma}_{i j}$

$$
\begin{aligned}
& \hat{\sigma}_{j j}(t) \longrightarrow \hat{\sigma}_{j j}(t), \\
& \hat{\sigma}_{12}(t) \longrightarrow \hat{\sigma}_{12}(t) e^{i\left(\omega_{L} t-\phi\right)},
\end{aligned}
$$

tal que a fase $\phi$ é definida de modo que $\left\langle\alpha_{L}\left|\hat{g}_{L}\right| \alpha_{L}\right\rangle \in \mathbb{R}$, ou seja, ela faz o papel da fase média do campo incidente. O novo sistema será

$$
\begin{aligned}
& \dot{\hat{\sigma}}_{22}=i \hat{g}_{L}^{\dagger} \hat{\sigma}_{12}(t)-i \hat{\sigma}_{21}(t) \hat{g}_{L}-\Gamma \hat{\sigma}_{22}(t)+\hat{F}_{22}(t), \\
& \dot{\hat{\sigma}}_{12}=\left(i \delta_{0}-\frac{\Gamma}{2}\right) \hat{\sigma}_{12}(t)+i\left[2 \hat{\sigma}_{22}(t)-1\right] \hat{g}_{L}+\hat{F}_{12}(t), \\
& \dot{\hat{\sigma}}_{21}=\left[\dot{\hat{\sigma}}_{12}\right]^{\dagger}
\end{aligned}
$$

onde $\delta_{0}=\omega_{L}-\omega_{0}$ é a dessintonia, $\hat{F}_{12}(t) \longrightarrow \hat{F}_{12}(t) e^{i \omega_{L} t}$ e $\hat{g}_{L} \longrightarrow \hat{g}_{L} e^{i \phi}$. Se ignorássemos este vínculo, a equação para os valores médios, que será mostrada na próxima seção, seria homogênea, portanto, somente possuiria solução identicamente nula. A eliminação de uma das equações nos permite chegar a um sistema livre de vínculos.

\subsection{Equações Óticas de Bloch}

Nesta seção, mostraremos que as equações para os valores médios são as previstas pela teoria semiclássica. Para isso, tomemos a média do sistema (3.23)-(3.26). O operador densidade no formalismo de Heisenberg é (3.12), então $\left\langle\hat{\sigma}_{i j}(t)\right\rangle=\operatorname{Tr}\left[\hat{\sigma}_{i j}(t) \rho_{T}\right]=\operatorname{Tr}\left[\hat{\sigma}_{i j} \rho_{T}(t)\right]$, tal que o traço é feito sobre todos os estados. Vamos expandir o modo laser na base de estados coerentes, os átomos na base $|n\rangle$ e os modos do vácuo numa base de estado número, cujo produto tensorial será escrito como $|N\rangle$. Desta forma,

$$
\operatorname{Tr}[\hat{A}]=\sum_{n,\{N\}} \frac{1}{\pi} \int_{S} d^{2} \alpha\langle\alpha ; n ; N|\hat{A}| \alpha ; n ; N\rangle
$$

onde $\{N\}$ representa o conjunto de todos $N_{i}$ de cada modo do vácuo, $S$ é a superfície no plano complexo e $\hat{A}$ é um operador qualquer que atua no sistema está definido no formalismo de Schrödinger, como $\rho_{T}(t)$. Então

$$
\begin{aligned}
& \left\langle\hat{\sigma}_{i j}(t)\right\rangle=\rho_{j i}(t) \\
& \left\langle\dot{\hat{\sigma}}_{i j}(t)\right\rangle=\dot{\rho}_{j i}(t),
\end{aligned}
$$

onde $\rho(t)=\operatorname{Tr}_{L, 0}\left[\rho_{T}(t)\right]=\frac{1}{\pi} \int_{S} d^{2} \alpha\left\langle\alpha ; 0\left|\rho_{T}(t)\right| \alpha ; 0\right\rangle$, é o operador densidade reduzido atômico, obtido por uma operação de traço parcial nos modos do vácuo e do campo. 
Esta operação, quando feita para o lado esquerdo das equações (3.39)-(3.41), fornecerá termos como $\operatorname{Tr}\left[\rho_{T} \hat{g}_{L}^{\dagger} \hat{\sigma}_{12}(t)\right]$ e $\operatorname{Tr}\left[\hat{\sigma}_{21}(t) \hat{g}_{L} \rho_{T}\right]$. Para o campo em um estado coerente, $\rho_{L}=\left|\alpha_{L}\right\rangle\left\langle\alpha_{L}\right|$, então $\hat{g}_{L} \rho_{L}=\Omega \rho_{L}$ e $\rho_{L} \hat{g}_{L}^{\dagger}=\Omega \rho_{L}$, onde

$$
\Omega=\left\langle\alpha_{L}\left|\hat{g}_{L}\right| \alpha_{L}\right\rangle
$$

é a frequência de Rabi. Sendo assim, o mesmo processo utilizado para derivar a média para $\sigma_{i j}(t)$ é válido para $\hat{g}_{L}^{\dagger} \hat{\sigma}_{12}(t)$ e $\hat{\sigma}_{21}(t) \hat{g}_{L}$, então

$$
\begin{aligned}
& \operatorname{Tr}\left[\rho_{T} \hat{g}_{L}^{\dagger} \hat{\sigma}_{12}(t)\right]=\Omega^{*} \rho_{21}(t), \\
& \operatorname{Tr}\left[\hat{\sigma}_{21}(t) \hat{g}_{L} \rho_{T}\right]=\Omega \rho_{12}(t) .
\end{aligned}
$$

As médias das forças de Langevin serão nulas, pois os outros modos estão no estado de vácuo. Portanto, as equações para os valores médios, usando o sistema (3.23)-(3.26), se tornam

$$
\begin{aligned}
& \dot{\rho}_{11}=\Gamma \rho_{22}(t)+i \Omega \rho_{12}(t) e^{-i \omega_{L} t}-i \Omega^{*} \rho_{21}(t) e^{i \omega_{L} t}, \\
& \dot{\rho}_{22}=-\Gamma \rho_{22}(t)-i \Omega \rho_{12}(t) e^{-i \omega_{L} t}+i \Omega^{*} \rho_{21}(t) e^{i \omega_{L} t}, \\
& \dot{\rho}_{12}=i \omega_{0} \rho_{12}(t)-\frac{\Gamma}{2} \rho_{12}(t)-i \Omega^{*}\left[\rho_{22}(t)-\rho_{11}(t)\right] e^{i \omega_{L} t}, \\
& \dot{\rho}_{21}=\left(\dot{\rho}_{12}\right)^{*} .
\end{aligned}
$$

Entretanto o estado do laser que usamos não é um estado coerente. Usamos um laser de diodo com um grande ruído de fase, cujo diagrama de Fresnel é mostrado na figura (3.3), mas o seu valor médio é o mesmo que um estado coerente. Desta forma, as diferenças existentes entre o laser de diodo e um estado coerente serão sentidas apenas nas flutuações, o que equivale à aproximação semiclássica [47]. Este raciocínio é bem razoável, pois, neste caso, a equação de movimento para os valores médios é a mesma satisfeista por um campo clássico, que corresponde a apenas um ponto no diagrama de Fresnel, sem a mancha que corresponde à flutuação. Para o caso de estados não-clássicos que são muito diferentes de estados coerentes, não poderíamos fazer a mesma análise, pois uma aproximação semiclássica não faria sentido.

O sistema (3.43)-(3.46) consiste das equações óticas de Bloch para a interação entre um campo eletromagnético clássico e um sistema de dois níveis [47, 46, 2]. Estas equações, que descrevem a dinâmica na aproximação semiclássica, são responsáveis pela evolução dos valores médios na teoria com o campo quantizado. Note que os termos dissipativos devidos à emissão espontânea, que entram na teoria semiclássica de forma fenomenológica, 


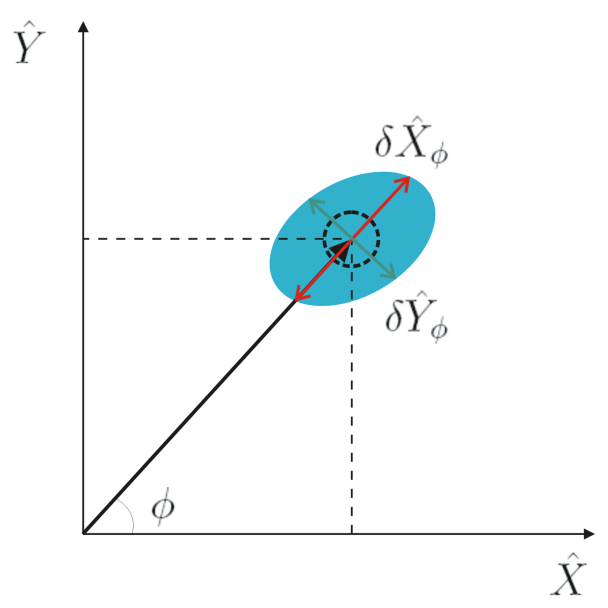

Figura 3.3: Diagrama de Fresnel para o laser de diodo. O círculo é o ruído de um estado coerente. A proporção entre o ruido de fase e de amplitude não está em escala, o fator pode chegar a 100 vezes. A proporção entre o valor médio e o ruído também não está em escala, pois este deveria ser bem menor que o valor médio.

aqui aparecem de forma natural como um efeito do acoplamento com os modos do vácuo. Podemos escrever o sistema de forma mais compacta se notarmos que os termos do lado direito de cada equação são os provenientes da equação de Von Neumann mais os termos dissipativos. Ou seja,

$$
\left.\frac{d \rho}{d t}=-\frac{i}{\hbar}[H, \rho]+\frac{\partial \rho}{\partial t}\right)_{d i s}
$$

com $H$ definido em (3.1), porém sem a parte do campo livre. $V$ está na RWA, mas agora a interação é feita com o campo clássico de amplitude $\mathbf{E}_{L}=\left|i \sqrt{2 \pi \hbar \omega_{L} / V} \alpha_{L} \mathbf{e}_{\mathbf{k}_{L} \lambda_{L}}\right|$. E o termo dissipativo pode ser compactado como

$$
\left.\frac{\partial \rho}{\partial t}\right)_{d i s}=\frac{\Gamma}{2}\left(2 \hat{\sigma}_{12} \rho \hat{\sigma}_{21}-\left\{\hat{\sigma}_{22}, \rho\right\}\right),
$$

tal que $\{$,$\} representa o anticomutador. Note que esta é a expressão da equação mestra$ para o sistema tratado com $\left.\frac{\partial \rho}{\partial t}\right)_{d i s}$ sendo o operador de Lindblad [48, 40].

O sistema (3.43)-(3.46) é bem mais simples de se resolver e, como mostrado em (3.35), também fornece a matriz de difusão. Usando o sistema (3.39)-(3.41), mostramos que

$$
\begin{aligned}
& \dot{\rho}_{22}=-\Gamma \rho_{22}(t)-i \Omega\left(\rho_{12}(t)-\rho_{21}(t)\right), \\
& \dot{\rho}_{12}=-\left(i \delta+\frac{\Gamma}{2}\right) \rho_{12}(t)-i \Omega\left[2 \rho_{22}(t)-1\right], \\
& \dot{\rho}_{21}=\left(\dot{\rho}_{12}\right)^{*}
\end{aligned}
$$

onde agora $\Omega \in \mathbb{R}$. Supondo que exista uma solução estacionária, $\rho_{i j} \longrightarrow \bar{\rho}_{i j}$ quando 
$t \longrightarrow \infty$, então $\dot{\bar{\rho}}_{i j} \longrightarrow 0$ e o sistema anterior é facilmente resolvido,

$$
\begin{aligned}
\bar{\rho}_{22} & =\Omega L(\delta) \\
\bar{\rho}_{12} & =L(\delta)\left(\delta+i \frac{\Gamma}{2}\right), \\
L(\delta) & =\frac{\Omega}{2 \Omega^{2}+\delta^{2}+\left(\frac{\Gamma}{2}\right)^{2}} .
\end{aligned}
$$

O índice de refração e a absorção dependerão da parte real e imaginária de $\rho_{12}$, respectivamente. As expressões para a absorção, o índice de refração e a diferença de população são

$$
\begin{aligned}
A b s(\delta) & =\Gamma L(\delta) \\
n(\delta) & =1+2 \delta L(\delta), \\
\Delta_{p}(\delta) & =2 \Omega L(\delta)-1,
\end{aligned}
$$

onde $\Delta_{p}=\bar{\rho}_{22}-\bar{\rho}_{11}$ é a diferença de população entre o estado excitado e o fundamental e a largura de $L(\delta)$ é dada por

$$
\Delta \omega=2 \sqrt{2 \Omega^{2}+\left(\frac{\Gamma}{2}\right)^{2}} .
$$

Como pode ser visto, para o campo muito fraco, $\Omega \ll 1, \Delta \omega=\Gamma$. Sendo assim, mesmo que dispusessemos de um laser completamente monomodo, não poderíamos obter uma resolução da frequência de transição atômica menor que $\Delta f=\Gamma / 2 \pi$ utilizando apenas um feixe. Este limite é chamado de largura natural e para o caso da linha $D 2$ do rubídio é $\Delta f \approx 6 M h z$. Note que à medida que $\Omega$ aumenta, menor se torna a resolução, o que é chamado de alargamento por potência, pois $\Omega$ é proporcional á intensidade do laser. Porém, outra forma de alargamento do sinal de absorção será mais importante ao fazer medidas em uma célula de vapor, o alargamento Doppler, que será tratado em detalhes na descrição do aparato experimental.

Os gráficos para $A b s(\delta), n(\delta)$ e $\Delta_{p}(\delta)$ são mostrados na Figura 3.4 com $\delta$ medida em unidades de $\Gamma$ e com $\Omega=\Gamma / 2$. Podemos ver que para $\delta$ muito pequena há um aumento na absorção e uma diminuição no índice de refração, e para a meia altura da lorentziana obtemos tanto o máximo quanto mínimo no índice de refração dependendo se a dessintonia é positiva ou negativa.

Este tipo de solução analítica geralmente não será aplicável para um sistema com mais de dois níveis, pois a quantidade de equações será bem maior. Sendo assim, a solução é 

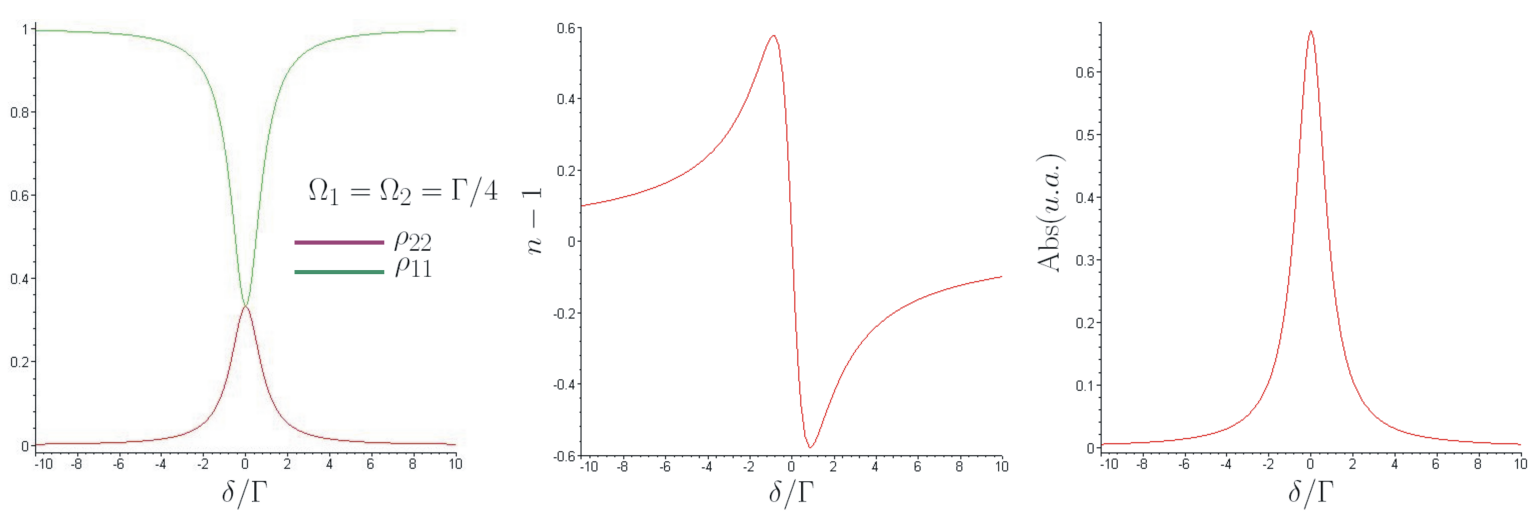

Figura 3.4: Diferença de população e população do estado excitado. Coeficiente de dispersão, que é proporcional ao indice de refração e coeficiente absorção.

geralmente realizada de forma numérica ou usando plataformas de computação simbólica como Maple e Mathematica.

Para deixar o sistema numa forma adequada para um tratamento numérico e justificar a existência da solução estacionária, desenvolveremos uma forma geral de chegar à solução para equações do tipo (3.49)-(3.51). Para isto, convém escrever a matriz densidade como um vetor, $\mathbf{X}(t)=\left(\rho_{22}(t), \rho_{12}(t), \rho_{21}(t)\right)^{T}$, onde usamos o vínculo $\rho_{11}(t)+\rho_{22}(t)=1$. $\mathbf{A}$ equação para $\mathbf{X}$ pode ser escrita como

$$
\frac{d \mathbf{X}}{d t}=\mathbf{A X}+\mathbf{B}
$$

onde $\mathbf{B}=(0, i \Omega,-i \Omega)^{T} \mathrm{e}$

$$
\mathbf{A}=\left[\begin{array}{ccc}
-\Gamma & -i \Omega & i \Omega \\
-2 i \Omega & -\left(i \delta+\frac{\Gamma}{2}\right) & 0 \\
2 i \Omega & 0 & \left(i \delta-\frac{\Gamma}{2}\right)
\end{array}\right]
$$

Essa equação é facilmente resolvida usando uma generalização do método de fator integrante. Podemos escrever

$$
\frac{d}{d t}\left(e^{-\mathbf{A} t} \mathbf{X}(t)\right)=e^{-\mathbf{A} t} \mathbf{B}
$$

onde $e^{-\mathbf{A} t}$ é definido como uma série de potências, assim como as funções de operadores na Mecânica Quântica, portanto

$$
\mathbf{X}(t)=e^{\mathbf{A} t}\left(\mathbf{X}(0)+\mathbf{A}^{-1} \mathbf{B}\right)-\mathbf{A}^{-1} \mathbf{B} .
$$

Desta forma, para garantir uma solução estacionária, a matriz $\exp (\mathbf{A} t)$ deve convergir para $t \rightarrow \infty$. Se $\mathbf{A}$ for diagonalizável, $\mathbf{S}^{-1} \mathbf{A} \mathbf{S}=\mathbf{D}$, onde $\mathbf{D}$ é uma matriz diagonal 
formada pelos autovalores de $\mathbf{A}$, então

$$
e^{\mathbf{A} t}=\mathbf{S} e^{\mathbf{D} t} \mathbf{S}^{-1}
$$

Como $\mathbf{S}$ não depende do tempo, a convergência de $\mathbf{D}$ garante a convergência de $\mathbf{A}$. Portanto, haverá uma solução estacionária quando a parte real de cada autovalor de A for negativa. Então

$$
\overline{\mathbf{X}}=-\mathbf{A}^{-1} \mathbf{B}
$$

que é a solução obtida quando $\dot{\mathbf{X}}(t)=0$. Esta solução é a que será medida no experimento e é independente do operador densidade inicial do sistema, representado por $\mathbf{X}(0)$. A condição que os autovalores de A sejam negativos será também condição de estabilidade das soluções para a flutuação, como discutido em [23]. Porém, antes de mais nada, esta é a condição para que haja um estado estacionário.

\subsection{Operador Polarização}

Como sugere a relação (3.10), o campo eletromagnético pode ser desmembrado em uma parte livre e uma parte que depende dos operadores atômicos. Neste seção, mostraremos que esta parte pode ser associada à polarização na região de interação. A componente de frequência positiva do campo de polarização para o modo laser, $\mathbf{k}_{L} \lambda_{L}$ é

$$
\hat{\mathbf{E}}_{p}^{(+)}(t)=i \frac{2 \pi \omega_{L}}{V} \mathbf{v}_{L} \int_{0}^{t} e^{-i \omega_{L}\left(t-t^{\prime}\right)} \hat{\sigma}_{12}\left(t^{\prime}\right) d t^{\prime}
$$

e realizando uma integração por partes chegamos a

$$
\hat{\mathbf{E}}_{L p}^{(+)}(t)=\frac{2 \pi}{V} \mathbf{v}_{L}\left[\hat{\sigma}_{12}(t)-e^{-i \omega_{L} t} \hat{\sigma}_{12}(0)-\int_{0}^{t} e^{-i \omega_{L}\left(t-t^{\prime}\right)} \dot{\hat{\sigma}}_{12}\left(t^{\prime}\right) d t\right] .
$$

O procedimento feito para derivar a taxa $\Gamma$ leva em consideração que todos os modos contribuem da mesma forma, o que é válido para a emissão espontânea. Porém, o átomo emite preferencialmete no modo do campo, com uma probabilidade que é a mesma para a emissão espontânea multiplicada pelo número de fótons naquele modo [27, 36]. Essa influência dos átomo no campo é a emissão estimulada e o campo de polarização calculado no modo laser é consequência da mesma. Realizando outra integração por partes no segundo termo, obtemos

$$
\hat{\mathbf{E}}_{L p}^{(+)}(t)=\frac{2 \pi}{V} \mathbf{v}_{L}\left\{\left[\hat{\sigma}_{12}(t)-e^{-i \omega_{L} t} \hat{\sigma}_{12}(0)\right]+\frac{i}{\omega_{L}}\left[\dot{\hat{\sigma}}_{12}(t)-e^{-i \omega_{L} t} \dot{\hat{\sigma}}_{12}(0)\right]+\int_{0}^{t} e^{-i \omega_{L}\left(t-t^{\prime}\right)} \ddot{\hat{\sigma}}_{12}\left(t^{\prime}\right) d t\right\} .
$$


Como as frequências estão no domínio ótico e são da ordem de $10^{14} \mathrm{~Hz}$, o segundo e o terceiro termos serão bem menores que o primeiro, pois as escalas temporais medidas são da ordem de milissegundos. Sendo assim, apenas o primeiro termo irá sobreviver. Se o átomo está no estado fundamental no instante inicial, $\rho_{A}=|1\rangle\langle 1|,\left\langle\hat{\sigma}_{12}(0)\right\rangle=\rho_{A ; 12}(0)$ e esta contribuição terá média nula, pois $\rho_{A ; 12}=0$. A contribuição deste termo para a correlação também será nula, pois como $\sigma_{12}(0) \rho_{A}=0,\left\langle\hat{\sigma}_{i j}(t) \hat{\sigma}_{12}(0)\right\rangle=\operatorname{Tr}\left[\hat{\sigma}_{i j}(t) \hat{\sigma}_{12}(0) \rho_{T}\right]=0$. Como os termos de ordem superior serão desprezados podemos escrever a componente de frequência positiva do operador polarização no modo laser como

$$
\hat{E}_{p L}^{(+)}(t)=d_{12} \frac{2 \pi}{V} \hat{\sigma}_{12}(t)
$$

onde $\hat{E}_{p L}^{(+)}(t)=\mathbf{e}_{L}^{*} \cdot \hat{\mathbf{E}}_{p L}^{(+)}(t)$ e $d_{12}=\mathbf{e}_{L}^{*} \cdot \mathbf{d}_{12}$

$$
\hat{P}^{(+)}(t)=\frac{d_{12}}{V} \hat{\sigma}_{12}(t)
$$

e a polarização total é $\hat{P}(t)=\hat{P}^{(+)}(t)+\hat{P}^{(-)}(t)$, onde $\hat{P}^{(-)}(t)=\left[\hat{P}^{(+)}(t)\right]^{\dagger}$. Como era esperado, a polarização média é proporcional à média do operador de dipolo. No caso em que o campo atua em um conjunto de átomos não interagentes, a polarização média na direção do campo será

$$
\langle\hat{P}(t)\rangle=n \operatorname{Tr}[\hat{d} \rho(t)]
$$

onde $n$ é a densidade de átomos.

Substituindo $\hat{\sigma}_{i j}(t)$ pelas componentes lentamente variáveis como em (3.37) e (3.38), chegamos a

$$
\left\langle\hat{P}^{(+)}(t)\right\rangle=n \hat{d}_{12} \rho_{21}(t) e^{-i\left(\omega_{L} t-\phi\right)},
$$

ou seja, $\left\langle\hat{P}^{(+)}(t)\right\rangle \sim\left\langle\hat{E}_{l L}^{(+)}(t)\right\rangle^{4}$. Com esta relação podemos mostrar que a que o meio é absortivo e dispersivo e que a dispersão e a absorção são, respectivamente, proporcionais às partes real e imaginária da suceptibilidade complexa [39]. Neste caso, as componentes do operador densidade farão o papel de suceptibilidade complexa e a expressão para o coeficiente de absorção e o índice de refração se tornam (3.52) e (3.53). Como o campo após deixar a amostra atômica terá sua amplitude diminuida devido à absorção, a diferença entre as amplitudes de saída e de entrada é proporcional à parte imaginária da suceptibilidade e à polarização, ou seja, o campo que sai da amostra para a deteção é

$$
\hat{E}_{\text {saída }}^{(+)}(t)=\hat{E}_{l}^{(+)}(t)-i \kappa \hat{P}^{(+)}(t)
$$

\footnotetext{
${ }^{4}$ Note que isto não quer dizer que o meio seja linear, pois a relação de proporcionalidade ocorre entra as amplitudes complexas e não entre a polarização e o campo propriamente ditos.
} 
onde $\kappa$ é uma constante de proporcionalidade real.

\subsection{Equações para as Flutuações Atômicas e Espectro de Ruído}

Como mostrado anteriormente, as equações de Heisenberg-Langevin adimitem solução para os valores médios. Nesta seção, obteremos um conjunto de equações linearizadas para as flutuações. Para um campo intenso, as flutuações presentes são bem menores que o valor médio, o que justifica a utilização da linearização. Apesar de o laser de diodo possuir um grande ruído de fase, é razoável que este seja pequeno em comparação ao valor médio, de modo que a aproximação seja válida ${ }^{5}$. Como as flutuações na polarização serão proporcionais às dos operadores atômicos, separaremos estes e o operador do campo em valores médios e flutuações,

$$
\begin{aligned}
\hat{\sigma}_{i j}(t) & =\rho_{j i}+\delta \hat{\sigma}_{i j}(t), \\
\hat{g}_{L} & =\Omega+\delta \hat{g}_{L}(t),
\end{aligned}
$$

onde $\delta \hat{g}_{L}(t)$ são as flutuações lentamente variáveis e $\bar{\rho}_{j i}$ é a solução para o estado estacionário. Já desprezados os produtos das flutuações, as equações para as mesmas serão

$$
\begin{aligned}
\frac{d}{d t} \delta \hat{\sigma}_{22} & =i\left[\left(\delta \hat{g}_{L}^{\dagger}\right) \bar{\rho}_{21}-\bar{\rho}_{12} \delta \hat{g}_{L}\right]+i \Omega\left[\delta \hat{\sigma}_{12}(t)-\delta \hat{\sigma}_{21}(t)\right]-\Gamma \delta \hat{\sigma}_{22}(t)+\hat{F}_{22}(t), \\
\frac{d}{d t} \delta \hat{\sigma}_{12} & =\left(i \delta_{0}-\frac{\Gamma}{2}\right) \delta \hat{\sigma}_{12}(t)+2 i \Omega \delta \hat{\sigma}_{22}(t)+i\left[2 \bar{\rho}_{22}-1\right] \delta \hat{g}_{L}+\hat{F}_{12}(t), \\
\frac{d}{d t} \delta \hat{\sigma}_{21} & =\left[\frac{d}{d t} \delta \hat{\sigma}_{12}\right]^{\dagger} .
\end{aligned}
$$

Definimos o vetor $\delta \hat{\mathbf{X}}=\left(\delta \hat{\sigma}_{11}, \delta \hat{\sigma}_{22}, \delta \hat{\sigma}_{21}, \delta \hat{\sigma}_{12}\right)^{T}$, tal que

$$
\frac{d}{d t} \delta \hat{\mathbf{X}}(t)=\mathbf{A} \delta \hat{\mathbf{X}}(t)+i\left[\left(\mathbf{N}_{-} \overline{\mathbf{X}}+\mathbf{b}_{-}\right) \delta \hat{g}_{L}(t)+\left(\mathbf{N}_{+} \overline{\mathbf{X}}+\mathbf{b}_{+}\right) \delta \hat{g}_{L}^{\dagger}(t)\right]+\hat{\mathbf{f}}(t) .
$$

O estado estacionário é ordenado como $\overline{\mathbf{X}}=\left(\bar{\rho}_{22}, \bar{\rho}_{12}, \bar{\rho}_{21}\right), \hat{\mathbf{f}}(t)$ são as forças de Langevin, cuja média e correlação são definida em (3.32)-(3.34), a matriz A é a mesma definida em

\footnotetext{
${ }^{5}$ Um modelo não-linearizado utilizando campos clássicos e onde a fase é tratado como uma variável estocástica é desenvolvido em [24, 23, 25].
} 
(3.57) e

$$
\mathbf{N}_{-}=\left[\begin{array}{ccc}
0 & -1 & 0 \\
0 & 0 & 0 \\
2 & 0 & 0
\end{array}\right], \quad \mathbf{b}_{-}=\left(\begin{array}{c}
0 \\
0 \\
-1
\end{array}\right), \quad \mathbf{N}_{+}=\left[\begin{array}{ccc}
0 & 0 & 1 \\
-2 & 0 & 0 \\
0 & 0 & 0
\end{array}\right], \quad \mathbf{b}_{+}=\left(\begin{array}{c}
0 \\
1 \\
0
\end{array}\right)
$$

Note que a condição de estabilidade para a flutuação é a mesma que para a existência da solução estacionária, $\operatorname{Re}\left(\lambda_{i}\right)>0$, onde $\lambda_{i}$ são os autovalores de A. Ambas as condições podem ser vistas como forma diferentes de ver a estabilidade, pois, a própria convergência do valor médio, por si só, já garante a estabilidade do sistema.

Como mostrado na seção anterior, a flutuação na componente de frequência positiva da polarização atômica polarizada na direção $\mu$ é $\delta \hat{P}_{\mu}^{(+)}(t)=\mathbf{e}_{\mu}^{*} \cdot \mathbf{d}_{12} \delta \hat{\sigma}_{12}(t)$, que pode ser escrita como

$$
\begin{aligned}
\delta \hat{P}_{\mu}^{(+)}(t) & =\mathbf{e}_{\mu}^{*} \cdot \mathbf{d}_{12} \mathbf{v}^{T} \delta \hat{\mathbf{X}}(t) \\
\mathbf{v} & =\left(\begin{array}{lll}
0 & 0 & 1
\end{array}\right)^{T}
\end{aligned}
$$

A componente de frequência negativa também pode ser escrita da mesma maneira, usando $\delta \hat{P}_{\mu}^{(+)}(t)=\mathbf{e}_{\mu} \cdot \mathbf{d}_{21} \delta \hat{\sigma}_{21}(t)$,

$$
\begin{aligned}
\delta \hat{P}_{\mu}^{(-)}(t) & =\mathbf{e}_{\mu} \cdot \mathbf{d}_{21} \mathbf{u}^{T} \delta \hat{\mathbf{X}}(t) \\
\mathbf{u} & =\left(\begin{array}{lll}
0 & 1 & 0
\end{array}\right)^{T}
\end{aligned}
$$

Podemos tomar o hermitiano conjugado de ambas expressões e chegar a uma forma alternativa para as mesmas

$$
\begin{aligned}
\delta \hat{P}_{\mu}^{(-)}(t) & =\mathbf{e}_{\mu} \cdot \mathbf{d}_{21} \delta \hat{\mathbf{X}}^{\dagger}(t) \mathbf{v}, \\
\delta \hat{P}_{\mu}^{(+)}(t) & =\mathbf{e}_{\mu}^{*} \cdot \mathbf{d}_{12} \delta \hat{\mathbf{X}}^{\dagger}(t) \mathbf{u},
\end{aligned}
$$

Passando para o domínio da frequência obtemos para a polarização

$$
\begin{aligned}
& \delta \hat{P}_{\mu}^{(-)}(\omega)=\mathbf{e}_{\mu} \cdot \mathbf{d}_{21} \delta \hat{\mathbf{X}}^{\dagger}(\omega) \mathbf{v}=\mathbf{e}_{\mu} \cdot \mathbf{d}_{21} \mathbf{u}^{T} \delta \hat{\mathbf{X}}(\omega) \\
& \delta \hat{P}_{\mu}^{(+)}(\omega)=\mathbf{e}_{\mu}^{*} \cdot \mathbf{d}_{12} \delta \hat{\mathbf{X}}^{\dagger}(\omega) \mathbf{u}=\mathbf{e}_{\mu}^{*} \cdot \mathbf{d}_{12} \mathbf{v}^{T} \delta \hat{\mathbf{X}}(\omega)
\end{aligned}
$$

e a equação (3.65) é facilmente resolvida,

$$
\delta \hat{\mathbf{X}}(\omega)=[i \omega-\mathbf{A}]^{-1}\left[i\left[\left(\mathbf{N}_{-} i \overline{\mathbf{X}}+\mathbf{b}_{-}\right) \delta \hat{g}_{L}(\omega)+\left(\mathbf{N}_{+} i \overline{\mathbf{X}}+\mathbf{b}_{+}\right) \delta \hat{g}_{L}^{\dagger}(\omega)\right]+\hat{\mathbf{f}}(\omega)\right] .
$$

As medidas que analizaremos serão de correlação de intensidade medidas por dois 
detetores no esquema de detecção balanceada, que será explicado na descrição dos métodos experimentais. A expressão teórica para os sinais de correlação de intensidade entre dois modos $\mu$ e $\mu^{\prime}$ é dada pela função de correlação de segunda ordem definida em (2.84), que reescrevemos adiante

$$
G_{\mu, \mu^{\prime}}^{(2)}\left(t, t^{\prime}\right)=\left\langle\hat{E}_{\mu}^{(-)}(t) \hat{E}_{\mu^{\prime}}^{(-)}\left(t^{\prime}\right) \hat{E}_{\mu^{\prime}}^{(+)}\left(t^{\prime}\right) \hat{E}_{\mu}^{(+)}(t)\right\rangle
$$

A dependência em $\mathbf{r}$ será desconsiderada, pois o esquema é montado de forma simétrica de modo que a correlação seja sempre calculada entre pontos pertencentes á mesma frente de onda. Substituiremos $\hat{E}_{\mu}^{(+)}(t)=E_{\mu}+\delta \hat{E}_{\mu}^{(+)}(t)^{6}$ e seu hermitiano conjugado em (3.75), também desconsideraremos os termos de ordem maior que dois nas flutuações. Obteremos então

$$
\begin{aligned}
G_{\mu, \mu^{\prime}}^{(2)}\left(t, t^{\prime}\right)= & \left|E_{\mu}\right|^{2}\left|E_{\mu^{\prime}}\right|^{2}+E_{\mu}^{*} E_{\mu^{\prime}}^{*}\left\langle\delta \hat{E}_{\mu^{\prime}}^{(+)}\left(t^{\prime}\right) \delta \hat{E}_{\mu}^{(+)}(t)\right\rangle+E_{\mu}^{*} E_{\mu^{\prime}}\left\langle\delta \hat{E}_{\mu^{\prime}}^{(-)}\left(t^{\prime}\right) \delta \hat{E}_{\mu}^{(+)}(t)\right\rangle \\
& +E_{\mu} E_{\mu^{\prime}}^{*}\left\langle\delta \hat{E}_{\mu}^{(-)}(t) \delta \hat{E}_{\mu^{\prime}}^{(+)}\left(t^{\prime}\right)\right\rangle+E_{\mu} E_{\mu^{\prime}}\left\langle\delta \hat{E}_{\mu}^{(-)}(t) \delta \hat{E}_{\mu^{\prime}}^{(-)}\left(t^{\prime}\right)\right\rangle \\
& +\left|E_{\mu^{\prime}}\right|^{2}\left\langle\delta \hat{E}_{\mu}^{(-)}(t) \delta \hat{E}_{\mu}^{(+)}(t)\right\rangle+\left|E_{\mu}\right|^{2}\left\langle\delta \hat{E}_{\mu^{\prime}}^{(-)}\left(t^{\prime}\right) \delta \hat{E}_{\mu^{\prime}}^{(+)}\left(t^{\prime}\right)\right\rangle .
\end{aligned}
$$

Ao passar para o domínio da frequência, o primeiro termo será proporcional a uma delta de Dirac e as funções de correlação se tornarão espectros de ruído, como mostrado no Apêndice C. Desprezaremos a última linha da equação anterior, pois se trata de correlações no mesmo tempo e resultará em contribuições nulas para o espectro de ruído ${ }^{7}$, que será

$$
S_{\mu, \mu^{\prime}}(\omega)=E_{\mu}^{*} E_{\mu^{\prime}}^{*}\left\langle\delta \hat{E}_{\mu^{\prime}}^{(+)}(\omega) \delta \hat{E}_{\mu}^{(+)}(-\omega)\right\rangle+E_{\mu}^{*} E_{\mu^{\prime}}\left\langle\delta \hat{E}_{\mu^{\prime}}^{(-)}(\omega) \delta \hat{E}_{\mu}^{(+)}(-\omega)\right\rangle+\text { c.c.. }
$$

Nesta expressão, encontramos as covariâncias $\left\langle\delta \hat{E}_{\mu^{\prime}}^{(+)}(\omega) \delta \hat{E}_{\mu}^{(+)}(-\omega)\right\rangle,\left\langle\delta \hat{E}_{\mu^{\prime}}^{(-)}(\omega) \delta \hat{E}_{\mu}^{(+)}(-\omega)\right\rangle$. Como o campo total, depois de passar pela amostra atômica, na polarização $\mu$ é $\hat{E}_{\mu}^{(+)}(\omega)=$ $\hat{E}_{i n, \mu}^{(+)}(\omega)+i \beta \hat{P}_{\mu}^{(+)}(\omega)$,

$$
\begin{aligned}
\left\langle\delta \hat{E}_{\mu^{\prime}}^{(+)}(\omega) \delta \hat{E}_{\mu}^{(+)}(-\omega)\right\rangle= & \left\langle\delta \hat{E}_{i n, \mu^{\prime}}^{(+)}(\omega) \delta \hat{E}_{i n, \mu}^{(+)}(-\omega)\right\rangle+i \beta\left\langle\delta \hat{E}_{i n, \mu^{\prime}}^{(+)}(\omega) \delta \hat{P}_{\mu}^{(+)}(-\omega)\right\rangle \\
+ & i \beta\left\langle\delta \hat{P}_{\mu^{\prime}}^{(+)}(\omega) \delta \hat{E}_{i n, \mu}^{(+)}(-\omega)\right\rangle-\beta^{2}\left\langle\delta \hat{P}_{\mu^{\prime}}^{(+)}(\omega) \delta \hat{P}_{\mu}^{(+)}(-\omega)\right\rangle,(3.7 \\
\left\langle\delta \hat{E}_{\mu^{\prime}}^{(-)}(\omega) \delta \hat{E}_{\mu}^{(+)}(-\omega)\right\rangle= & \left\langle\delta \hat{E}_{i n, \mu^{\prime}}^{(-)}(\omega) \delta \hat{E}_{i n, \mu}^{(+)}(-\omega)\right\rangle+i \beta\left\langle\delta \hat{E}_{i n, \mu^{\prime}}^{(-)}(\omega) \delta \hat{P}_{\mu}^{(+)}(-\omega)\right\rangle \\
- & i \beta\left\langle\delta \hat{P}_{\mu^{\prime}}^{(-)}(\omega) \delta \hat{E}_{i n, \mu}^{(+)}(-\omega)\right\rangle+\beta^{2}\left\langle\delta \hat{P}_{\mu^{\prime}}^{(-)}(\omega) \delta \hat{P}_{\mu}^{(+)}(-\omega)\right\rangle .(3.7
\end{aligned}
$$

\footnotetext{
${ }^{6}$ Supor que o valor médio do campo é independente do tempo é equivalente a assumir que este se encontra em um estado estacionário.

${ }^{7}$ Estes termos são os mesmos desprezados em (2.80) e devem se anular na aproximação em que a média é muito maior que a flutuação.
} 
Usando as relações (3.72)-(3.74) e pelo fato de que $\delta \hat{E}_{i n, \mu^{\prime}}^{(+)}(\omega)=i \sqrt{\frac{2 \pi \hbar \omega}{V}} \delta \hat{a}_{\mu}(\omega)$ e $\delta \hat{g}_{\mu}(\omega)=$ $i \sqrt{\frac{2 \pi \omega}{\hbar V}}\left(\mathbf{e}_{\mu} \cdot \mathbf{d}_{21}\right) \delta \hat{a}_{\mu}(\omega)$, todas as covariâncias serão determinadas em função das covariâncias das variáveis do feixe laser $\left\langle\delta \hat{a}_{\mu}(\omega) \delta \hat{a}_{\mu^{\prime}}(-\omega)\right\rangle,\left\langle\delta \hat{a}_{\mu}^{\dagger}(\omega) \delta \hat{a}_{\mu^{\prime}}(-\omega)\right\rangle,\left\langle\delta \hat{a}_{\mu}(\omega) \delta \hat{a}_{\mu^{\prime}}^{\dagger}(-\omega)\right\rangle \mathrm{e}$ $\left\langle\delta \hat{a}_{\mu}^{\dagger}(\omega) \delta \hat{a}_{\mu^{\prime}}^{\dagger}(-\omega)\right\rangle$ e das flutuações introduzidas pelos modos no vácuo, $\left\langle f_{i}(\omega) f_{j}^{\dagger}(-\omega)\right\rangle$. Se o feixe laser estivesse em um estado coerente e fossem medidas correlações somente no modo laser, apenas $\left\langle\delta \hat{a}_{L}(\omega) \delta \hat{a}_{L}^{\dagger}(-\omega)\right\rangle$, que seria o ruído quântico padrão, e $\left\langle f_{i}(\omega) f_{j}^{\dagger}(-\omega)\right\rangle$ seriam não-nulos.

Entretanto, em nossa medidas, o feixe laser é dividido em um divisor de feixes polarizante, portanto, os modos $\mu$ e $\mu^{\prime}$ serão diferentes,

$$
\begin{aligned}
& \hat{a}_{\mu}=\frac{1}{\sqrt{2}}\left(\hat{a}_{L}+\hat{a}_{0}\right), \\
& \hat{a}_{\mu^{\prime}}=\frac{1}{\sqrt{2}}\left(\hat{a}_{L}-\hat{a}_{0}\right),
\end{aligned}
$$

onde $\hat{a}_{0}$ representa qualquer modo que está no vácuo e entra pela outra porta do divisor de feixes $^{8}$. Para qualquer modo no vácuo $\delta \hat{a}_{0}|0\rangle=\langle 0| \delta \hat{a}_{0}^{\dagger}=0$ e como $\left[\hat{a}_{+}, \hat{a}_{-}^{\dagger}\right]=0$, podemos calcular as covariâncias de $\hat{a}_{ \pm}(\omega)$ com $\hat{a}_{+}(\omega)$ em função das variáveis do laser,

$$
\begin{aligned}
\left\langle\delta \hat{a}_{\mu}(\omega) \delta \hat{a}_{\mu^{\prime}}(-\omega)\right\rangle & =\frac{1}{2}\left\langle\delta \hat{a}_{L}(\omega) \delta \hat{a}_{L}(-\omega)\right\rangle, \\
\left\langle\delta \hat{a}_{\mu}^{\dagger}(\omega) \delta \hat{a}_{\mu^{\prime}}^{\dagger}(-\omega)\right\rangle & =\frac{1}{2}\left\langle\delta \hat{a}_{L}^{\dagger}(\omega) \delta \hat{a}_{L}^{\dagger}(-\omega)\right\rangle, \\
\left\langle\delta \hat{a}_{\mu}^{\dagger}(\omega) \delta \hat{a}_{\mu^{\prime}}(-\omega)\right\rangle & =\frac{1}{2}\left\langle\delta \hat{a}_{L}^{\dagger}(\omega) \delta \hat{a}_{L}(-\omega)\right\rangle, \\
\left\langle\delta \hat{a}_{\mu}(\omega) \delta \hat{a}_{\mu^{\prime}}^{\dagger}(-\omega)\right\rangle & =\frac{1}{2}\left\langle\delta \hat{a}_{L}^{\dagger}(\omega) \delta \hat{a}_{L}(-\omega)\right\rangle .
\end{aligned}
$$

Se o laser estiver em um estado coerente, todas estas covariâncias irão se anular e apenas o ruído introduzido pelas forças de Langevin vai contribuir. Isto resultará que nas expressões (3.77) e (3.78), apenas as funções de covariância $\left\langle\delta \hat{P}_{\mu^{\prime}}^{(+)}(\omega) \delta \hat{P}_{\mu}^{(+)}(-\omega)\right\rangle$ e $\left\langle\delta \hat{P}_{\mu^{\prime}}^{(-)}(\omega) \delta \hat{P}_{\mu}^{(+)}(-\omega)\right\rangle$ serão diferentes de zero, pois são as únicas que possuem covariâncias do tipo $\left\langle f_{i}(\omega) f_{j}^{\dagger}(-\omega)\right\rangle$. Sendo assim, usando (3.72), (3.73) e (3.74), chegamos a

$$
\begin{gathered}
\left\langle\delta \hat{E}_{\mu^{\prime}}^{(+)}(\omega) \delta \hat{E}_{\mu}^{(+)}(-\omega)\right\rangle=\beta^{2}\left(\mathbf{e}_{\mu^{\prime}}^{*} \cdot \mathbf{d}_{12}\right)\left(\mathbf{e}_{\mu}^{*} \cdot \mathbf{d}_{12}\right) \mathbf{v}^{T}[i \omega-\mathbf{A}]^{-1}\left\langle\hat{\mathbf{f}}(\omega) \hat{\mathbf{f}}^{\dagger}(-\omega)\right\rangle\left[i \omega+\mathbf{A}^{\dagger}\right]^{-1} \mathbf{u} \\
\left\langle\delta \hat{E}_{\mu^{\prime}}^{(-)}(\omega) \delta \hat{E}_{\mu}^{(+)}(-\omega)\right\rangle=-\beta^{2}\left(\mathbf{e}_{\mu^{\prime}} \cdot \mathbf{d}_{21}\right)\left(\mathbf{e}_{\mu}^{*} \cdot \mathbf{d}_{12}\right) \mathbf{u}^{T}[i \omega-\mathbf{A}]^{-1}\left\langle\hat{\mathbf{f}}(\omega) \hat{\mathbf{f}}^{\dagger}(-\omega)\right\rangle\left[i \omega+\mathbf{A}^{\dagger}\right]^{-1} \mathbf{u} .
\end{gathered}
$$

\footnotetext{
${ }^{8} \mathrm{O}$ divisor de feixes é tratado no capítulo sobre os métodos experimentais, junto com a deteção balanceada.
} 
Portanto, o espectro de ruído será

$$
\begin{aligned}
S_{\mu^{\prime}, \mu}(\omega) & =I \beta^{2}\left\{2 \operatorname{Re}\left(d_{12}^{\mu} d_{21}^{\mu^{\prime}}\right) \mathbf{u}^{T} \mathbf{D}(\omega) \mathbf{u}-\left[d_{12}^{\mu} d_{12}^{\mu^{\prime}} \mathbf{v}^{T} \mathbf{D}(\omega) \mathbf{u}+d_{21}^{\mu} d_{21}^{\mu^{\prime}} \mathbf{u}^{T} \mathbf{D}(\omega) \mathbf{v}\right]\right\}(3.8 \\
\mathbf{D}(\omega) & =-[i \omega-\mathbf{A}]^{-1}\left\langle\hat{\mathbf{f}}(\omega) \hat{\mathbf{f}}^{\dagger}(-\omega)\right\rangle\left[i \omega+\mathbf{A}^{\dagger}\right]^{-1}
\end{aligned}
$$

onde $d_{12}^{\mu}=\mathbf{e}_{\mu}^{*} \cdot \mathbf{d}_{12}, d_{21}^{\mu}=\mathbf{e}_{\mu} \cdot \mathbf{d}_{21}$ e $I=\left|E_{\mu}\right|^{2}=\left|E_{\mu^{\prime}}\right|^{2}$ é a intenssidade de cada feixe. O espectro de ruído (3.83) mede as correlações entre os modos $\mu$ e $\mu^{\prime}$ que chegam em cada detetor.

Se $|1\rangle$ e $|2\rangle$ forem estados hiperfinos, a diferenciação entre os modos $\mu$ e $\mu^{\prime}$ não será necessária. Se escrevemos a polarização do laser base esférica, o dipolo atômico será projetado também na base esférica. Como visto no capítulo 2, cada componente da base esférica acopla estados hiperfinos com $\Delta m=0, \pm 1$. No caso em que não haja um campo magnético para quebrar a degenerescência do níveis hiperfinos, estes estados são degenerados. Portanto, independentemente de como seja a separação no divisor de feixes, o que será observado é uma contrituição média de todos os subníveis Zeeman.

O espectro de ruído, definido em (3.76) será

$$
S(\omega)=I \alpha\left\{2 \mathbf{u}^{T} \mathbf{D}(\omega) \mathbf{u}-\left[\mathbf{v}^{T} \mathbf{D}(\omega) \mathbf{u} e^{i \phi}+\mathbf{u}^{T} \mathbf{D}(\omega) \mathbf{v} e^{-i \phi}\right]\right\}
$$

onde e $\chi=\left|\mathbf{e} \cdot \mathbf{d}_{12}\right|$ é a projeção do dipolo atômico na direção da polarização do feixe. De posse da matriz de difusão $\mathbf{D}(\omega)$ podemos calcular diversos resultados. O pricipal deles é o próprio espectro de ruído devido á correlação entre os detetores (3.85). Susbtituindo as expressões para $\mathbf{u}$ e $\mathbf{v}$, obtemos

$$
S(\omega)=I \alpha\left\{2 \mathbf{D}_{22}(\omega)-\left[\mathbf{D}_{32}(\omega)+\mathbf{D}_{23}(\omega)\right] \cos \phi-i\left[\mathbf{D}_{32}(\omega)-\mathbf{D}_{23}(\omega)\right] \sin \phi\right\}
$$

Calculamos este espectro de ruído usando o Maple para $\phi=0$ e o resultado é mostrado na figura 3.5. Podemos ver que não há regiões de anticorrelação, o ruído calculado é sempre positivo. Também podemos calcular o espectro de ruído observado na fluorescência. Como para este caso, podemos fazer a medida com apenas um detetor, a função de correlação utilizada será $G^{(1)}\left(t, t^{\prime}\right)$ para o mesmo modo. Portanto, o espectro de ruído será

$$
S_{f l u}(\omega)=\left\langle\delta \hat{E}^{(-)}(\omega) \delta \hat{E}^{(+)}(-\omega)\right\rangle
$$

Este espectro de ruído é proporcional a $\mathbf{D}_{22}(\omega)$ e é mostrado na figura 3.6. Neste gráfico, podemos ver que o sinal de correlação nunca fica negativo. Podemos ver também a existência de três lorenztianas que vão se junta ao pico central á medida que a frequência de Rabi diminui. 


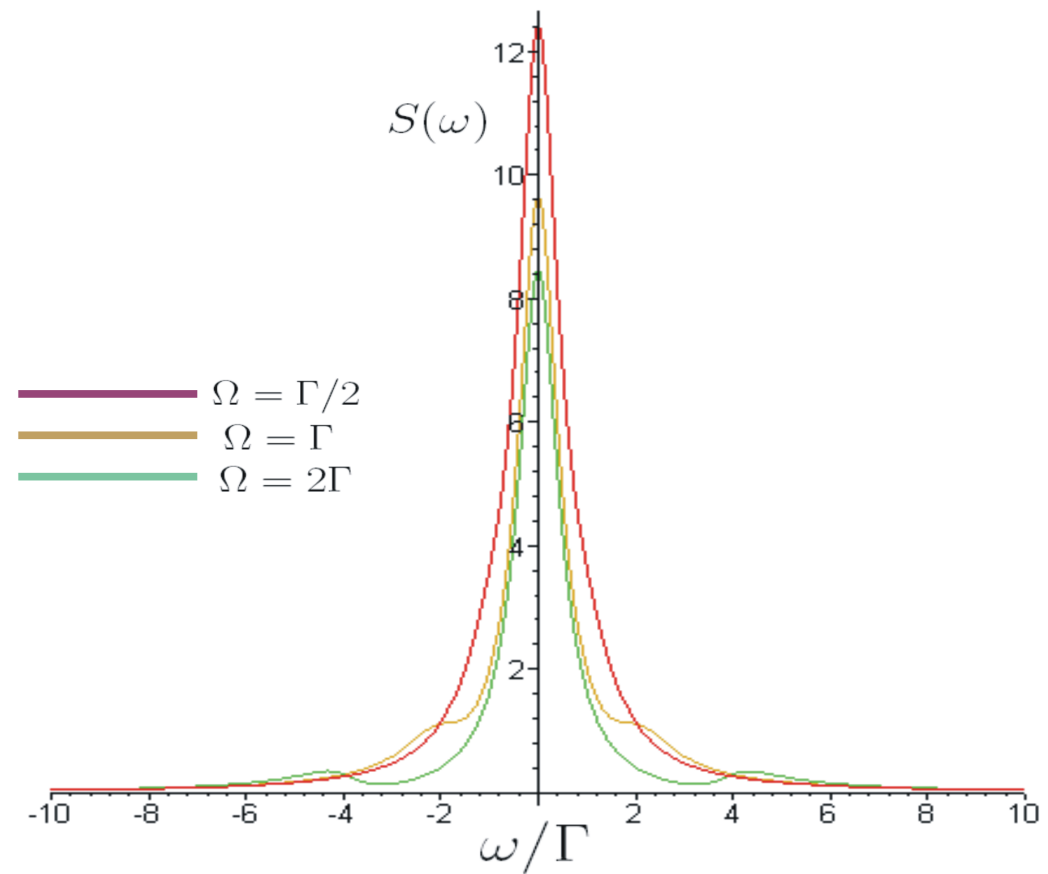

Figura 3.5: Espectro de Ruído para a correlação entre os dois detetores.

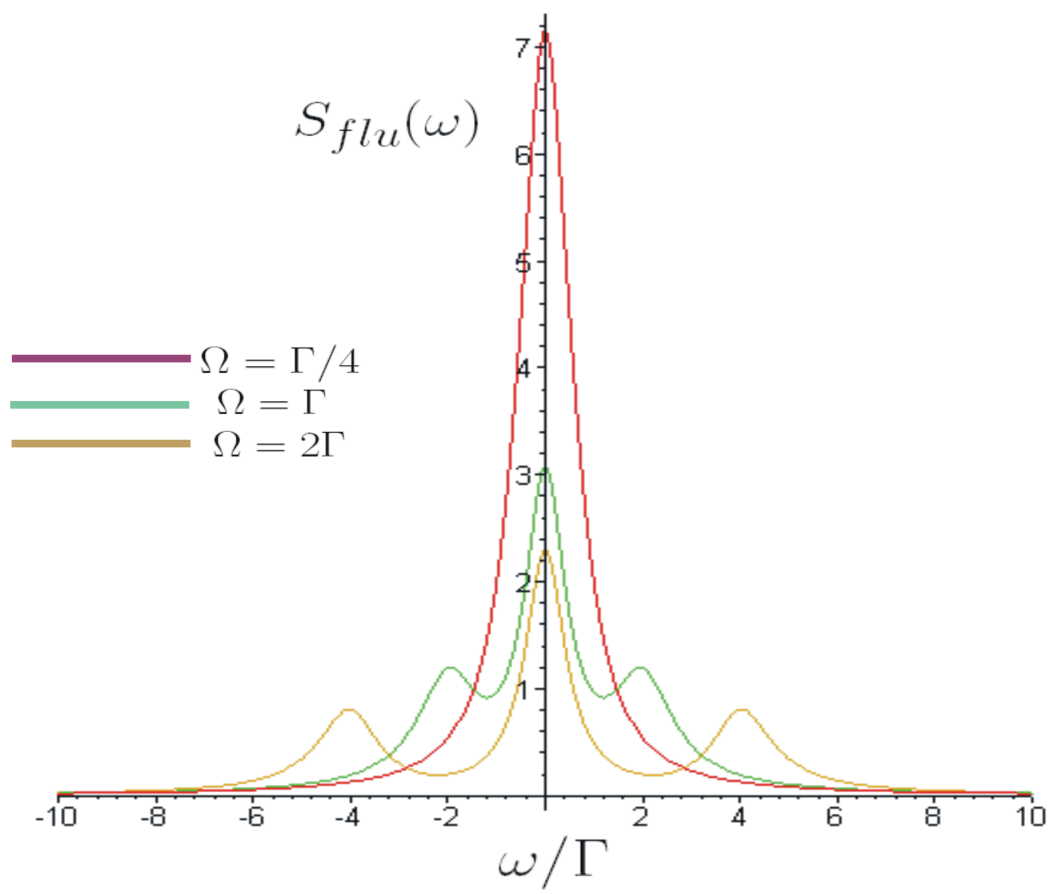

Figura 3.6: Espectro de Ruído na fluorescência para correlações medidas com apenas um detetor.

Ambos os resultados mostrados nos gráficos 3.5 e 3.6 são derivados supondo que o campo incidente esteja em um estado coerente. Caso isto não ocorra, as covariâncias mostradas em (3.79)-(3.82) serão não-nulas e todas irão contribuir para o sinal de $S_{\mu^{\prime}, \mu}(\omega)$. Para tratar este tipo de sistema precisamos saber o valor para as funções de covariância 
(3.79)-(3.82). Se conhecemos a matriz densidade do campo incidente, podemos calcular as covariâncias diretamente e determinar de qual o excesso de ruído presente no feixe. O mais comum é caracterizar o ruído por meio de uma medida direta, utilizando um esquema de deteção homodina ou uma cavidade ótica $[55,56]$. Para um feixe incidente com excesso de ruído, é geralmente mais simples fazer $\delta \hat{a}_{L}(t)=\hat{p}(t)+i \hat{q}(t)$, tal que $\hat{p}(t)$ e $\hat{q}(t)$ são hermitianos e serão as flutuações de fase e amplitude, respectivamente, caso o estado estacionário seja real. Esta será a abordagem feita no capítulo 5 para tratar o laser de diodo que possui um grande ruído de fase.

Neste capítulo analisamos a interação do campo quatizado com um átomo de dois níveis. Podemos ver vários fenômenos interessantes como o fato de o acoplamento do átomo com o reservatório gerar excesso de ruído no campo emitido, como mostrado nos gráficos 3.5 e 3.6. Porém, este tipo de sistema ainda é bastente limitado, pois o fato de haver apenas um estado fundamental impossibilita trocas de coerências entre estados fundamentais, transisões de dois fótons, mistura de quatro ondas e toda uma série de fenômenos interessantes. No próximo capítulo, trataremos do sistema mais simples onde estes fenômenos podem acontecer, o sistema de três níveis em $\Lambda$, e analisaremos especificamente o fenômeno da EIT. 


\section{EIT - Sistema de 3 Niveis}

Neste capítulo trataremos um sistema atômico de três níveis interegindo com dois campos eletromagnéticos. Neste tipo de sistema ocorrem vários fenômenos que não podem ser modelados por um sistema de dois níveis. Ele é o mais simples no qual pode haver fenômenos de laser sem inversão de população, interferência entre canais de excitação e vários outros fenômenos de CPT [40, 2]. Analisaremos o fenômeno da EIT de dois pontos de vista. No primeiro, utilizando estados vestidos, mostraremos a existência de um estado escuro e como a relação de fase dos campos e das transições atômicas podem fazer o átomo deixar de intragir com a radiação quando a mesma está ressonante. O segundo ponto de vista será utilizando as equações de Bloch para calcular o coeficiente de absorção e mostrar que realmente há uma queda na absorção quando os campos estão em ressonância. Neste capítulo, obteremos apenas a solução para os valores médios, um tratamento detalhado das correlações do campo ao interagir com este tipo de sistema pode ser encontrado em $[49,22,23,17]$.

\subsection{Formulação do Problema}

Considere o problema de dois campos quantizados interagindo ao mesmo tempo com um átomo. Cada campo está quase ressonante com uma transição, então podemos tratar o problema como um sistema de três níveis. Considere também que haja um estado excitado e dois fundamentais, ou seja, o átomo está na configuração- $\Lambda$ mostrada na figuura (4.1). O hamiltoniano total é

$$
H=H_{0}+H_{B}+V
$$

O hamiltoniano de campo livre, $H_{B}$, é o mesmo que no capítulo anterior e o hamiltoniano atômico será

$$
H_{0}=\sum_{j=1}^{3} \hbar \omega_{j} \hat{\sigma}_{j j} .
$$

onde $|2\rangle$ é o estado excitado e $|1\rangle$ e $|3\rangle$ são os estados fundamentais, com $\omega_{1}>\omega_{3}$. 


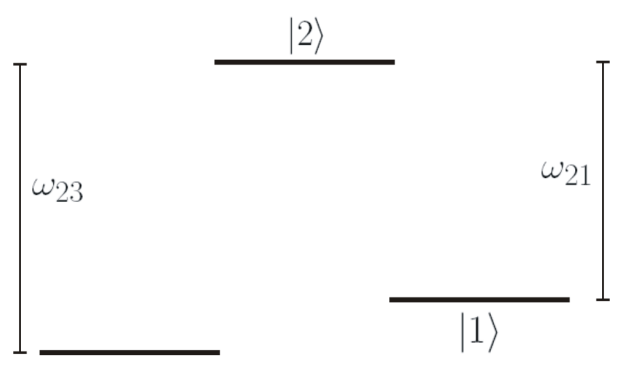

$|3\rangle$

Figura 4.1: Sistema de 3 Niveis

Como no sistema de dois níveis, usaremos como hamiltoniano de interação o hamitoniano de dipolo, apêndice A. Os níveis fundamentais são estados hiperfinos com mesmo momento angular total, que possuem a mesma paridade, ou seja, é uma transição proibida por dipolo. Portanto, além de $\langle i|\mathbf{d}| i\rangle=0$, também teremos $\langle 1|\mathbf{d}| 3\rangle=0$. Desta forma, na RWA,

$$
V=\left(\mathbf{d}_{12} \hat{\sigma}_{12}+\mathbf{d}_{32} \hat{\sigma}_{32}\right) \cdot \hat{\mathbf{E}}^{(-)}+\left(\mathbf{d}_{21} \hat{\sigma}_{21}+\mathbf{d}_{23} \hat{\sigma}_{23}\right) \cdot \hat{\mathbf{E}}^{(+)} .
$$

Com $\hat{\mathbf{E}}^{( \pm)}$dados em (2.66).

\subsection{Campos em Ressonância - Estado Escuro}

Começaremos o tratamento considerando os campos quase ressonantes com as transições e utilizaremos apenas um modo do campo acoplado a cada transição. O problema de se fazer essa suposição, como visto no capítulo anterior, é que desprezamos os termos responsáveis pela emissão espontânea. A grande vantagem de seguir este caminho alternativo ao traçado anteriormente é que, neste tratamento, a EIT pode ser vista de uma forma simples como um fenômeno de interferência destrutiva. Neste caso, o estado do campo é composto por dois campos de frequências distintas. Então, o vetor de estado é

$$
|\psi\rangle=|i\rangle \otimes\left|n_{1}\right\rangle \otimes\left|n_{2}\right\rangle
$$

onde $n_{1}$ é o estado número referente ao campo quase ressonante com a transição $|1\rangle \longrightarrow|2\rangle$ e $n_{2}$, com $|3\rangle \longrightarrow|2\rangle$. Os campos 1 e 2 são

$$
\hat{\mathbf{E}}_{j}^{(+)}=i \sqrt{\frac{\hbar \omega_{L j}}{2 V}} \hat{a}_{j} \mathbf{e}_{j}
$$


e o hamiltoniano (4.2) será

$$
\begin{aligned}
V & =\hbar g_{1} \hat{a}_{1} \hat{\sigma}_{21}+\hbar g_{2} \hat{a}_{2} \hat{\sigma}_{23}+h . c \\
g_{1} & =i \sqrt{\frac{\omega_{1}}{2 \hbar V}} \mathbf{e}_{1} \cdot \mathbf{d}_{21} \cdot \\
g_{2} & =i \sqrt{\frac{\omega_{2}}{2 \hbar V}} \mathbf{e}_{2} \cdot \mathbf{d}_{23} .
\end{aligned}
$$

No formalismo de interação a equação de movimento e a expressão para $V$ são

$$
\begin{aligned}
i \hbar \frac{\partial}{\partial t}\left|\psi_{I}\right\rangle & =V_{R W A}^{I}(t)\left|\psi_{I}\right\rangle, \\
V^{I} & =\hbar g_{1} \hat{a}_{1} \hat{\sigma}_{21} e^{-i \delta_{1} t}+\hbar g_{2} \hat{a}_{2} \hat{\sigma}_{23} e^{-i \delta_{2} t}+h . c
\end{aligned}
$$

onde $\delta_{1}=\omega_{L 1}-\omega_{21}$ e $\delta_{2}=\omega_{L 2}-\omega_{23}$ são as dessintonias dos campos 1 e 2 . Para $\delta_{i}=0$, $V^{I}$ não depende do tempo, então a equação (4.8) é resovida,

$$
\left|\psi_{I}(t)\right\rangle=e^{-\frac{i}{\hbar} V_{R W A}^{I} t}|\psi(0)\rangle
$$

Sendo assim, basta diagonalizar $V^{I}$ para obter os autoestados do sistema. O elemento de matriz na base (4.3) é

$$
\begin{aligned}
\left\langle i, n_{1}, n_{2}\left|V^{I}\right| j, n_{1}^{\prime}, n_{2}^{\prime}\right\rangle= & \hbar g_{1} \sqrt{n_{1}^{\prime}} \delta_{n_{1} n_{1}^{\prime}-1} \delta_{1 j} \delta_{2 i}+\hbar g_{2} \sqrt{n_{2}^{\prime}} \delta_{n_{2} n_{2}^{\prime}-1} \delta_{3 j} \delta_{2 i} \\
& +\hbar g_{1}^{*} \sqrt{n_{1}^{\prime}+1} \delta_{n_{1} n_{1}^{\prime}+1} \delta_{1 i} \delta_{2 j}+\hbar g_{2}^{*} \sqrt{n_{2}^{\prime}+1} \delta_{n_{2} n_{2}^{\prime}+1} \delta_{3 i} \delta_{2 j}
\end{aligned}
$$

A matriz $V^{I}$ será bloco diagonal no subespaço $\left|2, n_{1}-1, n_{2}-1\right\rangle,\left|1, n_{1}, n_{2}-1\right\rangle$ e $\mid 3, n_{1}-$ $\left.1, n_{2}\right\rangle$. Desta forma, cada bloco será

$$
V_{n_{1} n_{2}}^{I}=\left[\begin{array}{ccc}
0 & g_{1} \sqrt{n_{1}} & g_{2} \sqrt{n_{2}} \\
g_{1}^{*} \sqrt{n_{1}} & 0 & 0 \\
g_{2}^{*} \sqrt{n_{2}} & 0 & 0
\end{array}\right]
$$

Ao diagonalizar esta matriz, obtemos os autovalores $\lambda_{D}=0$ e $\lambda_{ \pm}= \pm \hbar \Omega$, com $\Omega=\sqrt{\left|\Omega_{1}\right|^{2}+\left|\Omega_{2}\right|^{2}}$, onde $\Omega_{i}$ são as frequências de Rabi referente ao campo $i$, definidas como

$$
\Omega_{i}=g_{i} \sqrt{n_{i}}
$$

Desta forma, os autoestados do sistema serão

$$
\begin{aligned}
\left|\psi_{D}^{n_{1}, n_{2}}\right\rangle & =\frac{\Omega_{1}\left|1, n_{1}, n_{2}-1\right\rangle-\Omega_{2}\left|3, n_{1}-1, n_{2}\right\rangle}{\Omega}, \\
\left|\psi_{ \pm}^{n_{1}, n_{2}}\right\rangle & =\frac{1}{\sqrt{2}}\left[\left|2, n_{1}-1, n_{2}-1\right\rangle \pm\left|\psi_{A}^{n_{1}, n_{2}}\right\rangle\right]
\end{aligned}
$$


com o estado acoplado, $\left|\psi_{A}^{n_{1}, n_{2}}\right\rangle$, definido como

$$
\left|\psi_{A}^{n_{1}, n_{2}}\right\rangle=\frac{\Omega_{2}^{*}\left|1, n_{1}, n_{2}-1\right\rangle+\Omega_{1}^{*}\left|3, n_{1}-1, n_{2}\right\rangle}{\sqrt{\left|\Omega_{1}\right|^{2}+\left|\Omega_{2}\right|^{2}}} .
$$

Como $V^{I}\left|\psi_{D}^{n_{1}, n_{2}}\right\rangle$ se anula, o estado $\left|\psi_{D}^{n_{1}, n_{2}}\right\rangle$ não se acopla com o campo, por isso é chamado de estado escuro ${ }^{1}$. Caso o átomo esteja nesta superposição dos estados fundamentais, ele será transparente à luz. O mesmo não ocorre para um sistema de dois níveis, onde há somente um estado fundamental, então não há como ter combinações que não interagem com o campo. Podemos inverter as relações (4.12)-(4.13) para obter

$$
\begin{aligned}
\left|2, n_{1}-1, n_{2}-1\right\rangle & =\frac{1}{\sqrt{2}}\left[\left|\psi_{+}^{n_{1}, n_{2}}\right\rangle+\left|\psi_{-}^{n_{1}, n_{2}}\right\rangle\right], \\
\left|1, n_{1}, n_{2}-1\right\rangle & =\frac{\Omega_{1}^{*}\left|\psi_{D}^{n_{1}, n_{2}}\right\rangle+\Omega_{2}\left|\psi_{A}^{n_{1}, n_{2}}\right\rangle}{\Omega}, \\
\left|3, n_{1}-1, n_{2}\right\rangle & =\frac{\Omega_{1}\left|\psi_{A}^{n_{1}, n_{2}}\right\rangle-\Omega_{2}^{*}\left|\psi_{D}^{n_{1}, n_{2}}\right\rangle}{\Omega} .
\end{aligned}
$$

Se o estado inicial é uma superposição dos estados fundamentais, $|\psi(0)\rangle=c_{1} \mid 1, n_{1}, n_{2}-$ $1\rangle+c_{3}\left|3, n_{1}-1, n_{2}\right\rangle$, então

$$
|\psi(0)\rangle=c_{D}\left|\psi_{D}^{n_{1}, n_{2}}\right\rangle+c_{A}\left|\psi_{A}^{n_{1}, n_{2}}\right\rangle,
$$

onde $c_{D}=\left(c_{1} \Omega_{1}^{*}-c_{3} \Omega_{2}^{*}\right) / \Omega$ e $c_{A}=\left(c_{1} \Omega_{2}+c_{3} \Omega_{1}\right) / \Omega$. Primeiramente, veremos em que condições $c_{A}=0$, então

$$
\left|c_{1}\right|\left|\Omega_{2}\right| e^{i\left(\phi_{2}+\psi_{1}\right)}=\left|c_{3}\right|\left|\Omega_{1}\right| e^{i\left(\phi_{1}+\psi_{3}+\pi\right)}
$$

onde $\phi_{i}$ é a fase de cada campo definido por $\Omega_{i}, \psi_{1}$ é a fase de $c_{1}$ e $\psi_{3}$ é a fase de $c_{3}$. Então,

$$
\begin{aligned}
\frac{\left|\Omega_{1}\right|}{\left|c_{1}\right|} & =\frac{\left|\Omega_{2}\right|}{\left|c_{3}\right|}, \\
\Delta \phi-\Delta \psi & =\pi,
\end{aligned}
$$

com $\Delta \phi=\phi_{1}-\phi_{2}$ e $\Delta \psi=\psi_{1}-\psi_{3}$. A primeira igualdade nos diz que, para que o estado inicial seja o estado escuro, a intensidade dos campos deve ser proporcinal à população dos níveis que estes acoplam com o estado excitado. Mas olhando para a segunda igualdade vemos que somente isto não é suficiente, há uma relação de fase que também deve ser

\footnotetext{
${ }^{1}$ Esta donominação foi utilizada em [3] como ressonância escura, pois o átomo parava de emitir flurescência em certas regiões. A existencia desse estado desacoplado foi a explicação proposta para esse fenômeno, por isso este é chamado de estado escuro.
} 
satisfeita. Se impomos somente a primeira igualdade,

$$
c_{A}=\frac{c_{1} \Omega_{2}}{\Omega}\left[1+e^{i(\Delta \phi-\Delta \psi)}\right] .
$$

Neste ponto fica mais fácil ver o processo de interferência que origina a EIT. Quando $\Delta \phi-\Delta \psi=k \pi$, com $k \in \mathbb{Z}$, o sistema está no estado escuro, mas ao mudar a fase dos campos para que $\Delta \phi-\Delta \psi=2 k \pi$ ainda haverá uma contribuição do estado acoplado e o átomo não será transparente ao campo. Deste ponto, a EIT pode ser vista como a intereferência destrutiva entre os caminhos possíveis de excitação, que é assegurada pela proporção entre as populações e intensidades, (4.19), e pela relação de fase apropriada, $(4.21)$.

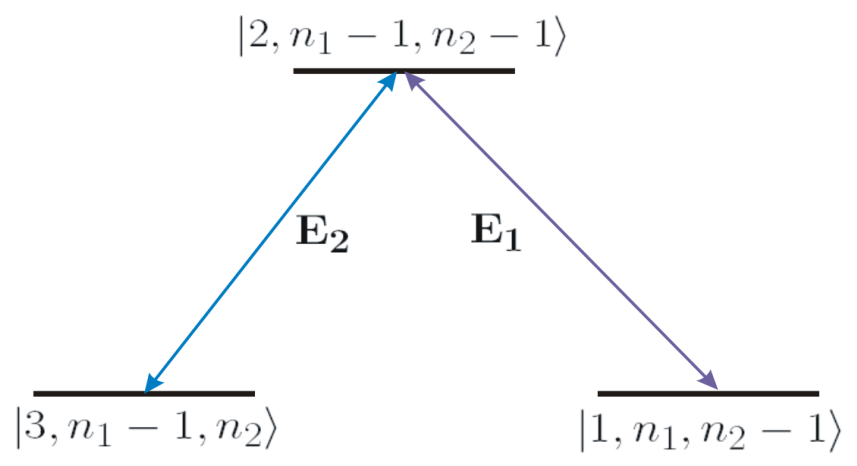

Figura 4.2: Sistema de Três nivies, onde se pode ver o processo de interferência dos dois canais de excitação.

Pelo fato de o estado escuro não se acoplar ao campo, a evolução temporal no formalismo de interação não irá afetá-lo. A evolução no hamiltoniano livre será

$$
\left|\psi_{D}^{n_{1}, n_{2}}(t)\right\rangle=\frac{1}{\Omega}\left[\Omega_{1} e^{-i\left[\omega_{1}+n_{1} \omega_{L 1}+\left(n_{2}-1\right) \omega_{L 2}\right] t}\left|1, n_{1}, n_{2}-1\right\rangle-\Omega_{2} e^{-i\left[\omega_{3}+\left(n_{1}-1\right) \omega_{L 1}+n_{2} \omega_{L 2}\right] t}\left|3, n_{1}-1, n_{2}\right\rangle\right] .
$$

Como $\omega_{L i}=\omega_{2}-\omega_{i}$, a evolução do estado escuro no hamiltoniano livre será apenas uma fase absoluta, portanto,

$$
\left|\psi_{D}^{n_{1}, n_{2}}(t)\right\rangle=\left|\psi_{D}^{n_{1}, n_{2}}(0)\right\rangle .
$$

Sendo assim, sempre que o estado inicial for preparado no estado escuro, as relações (4.20) e (4.19) farão com que este continue no estado escuro.

Vamos analisar agora o caso em que o estado inicial não é preparado no estado escuro. Fazendo a evolução de (4.18) e voltando à base (4.3) obtemos

$$
|\psi(t)\rangle=c_{D}\left|\psi_{D}^{n_{1}, n_{2}}\right\rangle+c_{A}\left[\cos (\Omega t)\left|\psi_{A}^{n_{1}, n_{2}}\right\rangle-\sin (\Omega t)\left|2, n_{1}-1, n_{2}-1\right\rangle\right] .
$$

Como visto em (4.18), podemos representar o estado fundamental na base $\left(\left|\psi_{D}^{n_{1}, n_{2}}\right\rangle,\left|\psi_{a}^{n_{1}, n_{2}}\right\rangle\right)$ 
ao invés de $\left(\left|1, n_{1}, n_{2}-1\right\rangle,\left|3, n_{1}-1, n_{2}\right\rangle\right)$. Caso o átomo esteja inicialmente em qualquer combinação com $c_{A} \neq 0$, pelo menos uma das relações (4.19) e (4.21) não foi satisfeita. Neste caso, haverá uma oscilação entre esta componente e o estado excitado com frequência $\Omega$. Na nova base o sistema se torna um sistema de dois níveis efetivo, pois o campo somente acompla um dos níveis fundamentais ao estado excitado.

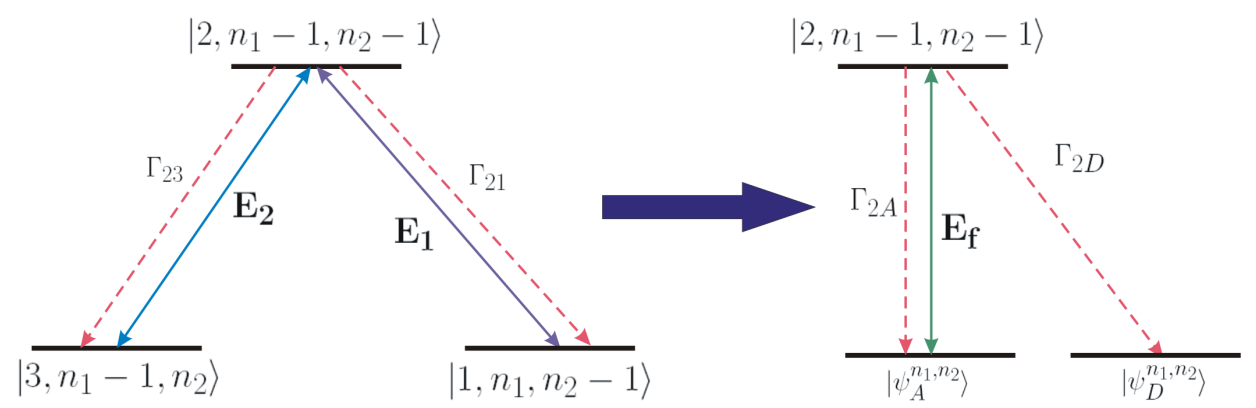

Figura 4.3: Trasnformação dos estados fundamentais para cada bloco.

Pelo fato de termos usado somente um modo do campo, a emissão espontânea não aparece neste modelo. Intoduzimos ela, por hora fenomenologiacamente, para analisar outro efeito importante para o fenômeno da EIT. Como pode ser visto na figura (4.3), o sistema de três níveis, na nova base, passa a ser um sistema de dois níveis aberto se consideramos a emissão espontânea. Então, além da interferência destrutiva dos canais de excitação, ocorrerá o bombeamento ótico para o estado escuro. Considerando que a emissão espontânea ocorra a taxas muito próximas para ambos os estados fundamentais, o átomo irá absorver um fóton do estado acoplado, ir para o estado excitado e poderá emitir para qualquer um dos fundamentais. Depois de vários ciclos deste processo, ocorrerá um acúmulo de população no estado escuro. Portanto, caso haja um estado estacionário o átomo se manterá em um estado que satisfaça as relações (4.19) e (4.20) independentemente dos valores para as frequências de Rabi.

Esse modelo foi resolvido diretamente utilizando os campos em ressonância com as transições. Porém ele é pouco reprodutível na prática, visto que em sistemas reais a emissão espontânea deve ser considerada de forma mais rigorosa e a ressonância exata é algo muito difícil de ser alcançado. Por exemplo, numa célula de vapor a distribuição de velocidades fará com que cada átomo, por efeito doppler, enxergue o campo com uma frequência diferente, portanto, quando alguns átomo estiverem em ressonância, outros não estarão. Por esses motivos, se faz necessário resolver o problema dependente do tempo e considerando dessintonias não-nulas. 


\subsection{Equações de Bloch}

O caminho natural para levar em conta o efeito de dessintonias não nulas e da emissão espontânea é utilizando as equações óticas de Bloch. Elas foram demonstradas na seção anterior como sendo a média das equações de Heisenberg-Langevin. Resolvendo a equação de Heisenberg, como em (3.13),

$$
\hat{a}_{\mathbf{k} \lambda}(t)=e^{-i \omega t} \hat{a}_{\mathbf{k} \lambda}-\sqrt{\frac{\omega}{2 V \hbar}} \int_{0}^{t} e^{-i \omega\left(t-t^{\prime}\right)} \mathbf{e}_{\mathbf{k} \lambda}^{*} \cdot\left[\mathbf{d}_{12} \hat{\sigma}_{12}\left(t^{\prime}\right)+\mathbf{d}_{32} \hat{\sigma}_{32}\left(t^{\prime}\right)\right] d t^{\prime},
$$

portanto, $\hat{\mathbf{E}}_{p}^{(+)}(t)$ possuirá duas contribuições, que darão origem a duas taxas de emissão espontânea, uma para cada estado fundamental. Ao fazer a substituição de $\hat{\mathbf{E}}_{p}^{( \pm)}(t)$ e $\hat{\mathbf{E}}_{l}^{( \pm)}(t)$ na ordem normal, chegamos ao conjunto de equações de Heisenberg-Langevin como em (3.23)-(3.23),

$$
\begin{aligned}
& \dot{\hat{\sigma}}_{11}=-i \hat{g}_{L 1}^{\dagger} e^{i \omega_{L 1} t} \hat{\sigma}_{12}+i \hat{\sigma}_{21} \hat{g}_{L 1} e^{-i \omega_{L 1} t}+\Gamma_{21} \hat{\sigma}_{11}+\hat{F}_{11}, \\
& \dot{\hat{\sigma}}_{22}=i \hat{g}_{L 1}^{\dagger} e^{i \omega_{L 1} t} \hat{\sigma}_{12}+i \hat{g}_{L 2}^{\dagger} e^{i \omega_{L 2} t} \hat{\sigma}_{32}+h . c .-\left(\Gamma_{21}+\Gamma_{23}\right) \hat{\sigma}_{22}+\hat{F}_{22}, \\
& \dot{\hat{\sigma}}_{33}=-i \hat{g}_{L 2}^{\dagger} e^{i \omega_{L 2} t} \hat{\sigma}_{32}+i \hat{\sigma}_{23} \hat{g}_{L 2} e^{-i \omega_{L 2} t}+\Gamma_{23} \hat{\sigma}_{11}+\hat{F}_{33}, \\
& \dot{\hat{\sigma}}_{12}=-i \omega_{21} \hat{\sigma}_{12}+i\left(\hat{\sigma}_{22}-\hat{\sigma}_{11}\right) \hat{g}_{L 1} e^{-i \omega_{L 1} t}-i \hat{\sigma}_{13} \hat{g}_{L 2} e^{-i \omega_{L 2} t}-\frac{\Gamma_{21}+\Gamma_{23}}{2} \hat{\sigma}_{21}+\hat{F}_{12}, \\
& \dot{\hat{\sigma}}_{32}=-i \omega_{23} \hat{\sigma}_{32}+i\left(\hat{\sigma}_{22}-\hat{\sigma}_{33}\right) \hat{g}_{L 2} e^{-i \omega_{L 2} t}-i \hat{\sigma}_{31} \hat{g}_{L 2} e^{-i \omega_{L 1} t}-\frac{\Gamma_{21}+\Gamma_{23}}{2} \hat{\sigma}_{23}+\hat{F}_{32}, \\
& \dot{\hat{\sigma}}_{13}=-i\left(\omega_{21}-\omega_{23}\right) \hat{\sigma}_{13}-i \hat{g}_{L 2}^{\dagger} \hat{\sigma}_{12} e^{i \omega_{L 2} t}+i \hat{\sigma}_{23} \hat{g}_{L 1} e^{-i \omega_{L 1} t}-\gamma \hat{\sigma}_{13}+\hat{F}_{13}, \\
& \dot{\hat{\sigma}}_{12}=\dot{\hat{\sigma}}_{21}^{*}, \\
& \dot{\hat{\sigma}}_{32}=\dot{\hat{\sigma}}_{23}^{*}, \\
& \dot{\hat{\sigma}}_{31}=\dot{\hat{\sigma}}_{13}^{*}
\end{aligned}
$$

tal que o operador densidade do sistema agora é

$$
\rho_{T}=\rho_{A} \otimes \rho_{L 1} \otimes \rho_{L 2} \otimes \rho_{0}
$$

Em princípio, ao fazer a substiuição de $\hat{\mathbf{E}}_{l}^{( \pm)}(t)$ haveria termos cruzados, como a interação do campo ressonante com $|1\rangle \longrightarrow|2\rangle$ definido por $\hat{g}_{L 1}$ com a transição $|3\rangle \longrightarrow|2\rangle$. Esses termos, que aparecem mesmo com campos clássicos partindo do hamiltoniano (A.7), serão desprezados, pois violam a condição de quase ressonância usada neste trabalho.

O fator $\gamma$ é uma taxa de decaimento entre os estados fundamentais proibida por dipolo, tal que $\gamma \ll \Gamma_{1 j}$. Ela pode ser um decaimento por colisão, efeito da entrada e saída de átomos da região onde está o feixe ou qualquer fenômeno que introduza troca de coerência entre os estados fundamentais. Sendo assim, essa taxa entrará na teoria de forma 
fenomenológica. Em pricípio, podemos adicionar uma taxa de decaimento não radiativa em todos os $\dot{\hat{\sigma}}_{i j}$. Mas como todos eles, a não ser $\dot{\hat{\sigma}}_{13}$, já possuem termos dissipativos adivindos de transissões radiativas, que são tipicamente muito maiores que os gerados por outros efeitos, consideramos somente um $\gamma$ atuando em $\dot{\hat{\sigma}}_{13}$.

Daqui em diante, usaremos somente uma taxa de emissão espontênea, ou seja, $\Gamma_{21}=$ $\Gamma_{23}=\Gamma$. Podemos ver em (3.27) que, em princípio, elas são diferentes, pois $\omega_{21} \neq \omega_{23}$ e $\left|\mathbf{d}_{21}\right| \neq\left|\mathbf{d}_{23}\right|$. Porém os níveis que estamos utilizando como níveis fundamentais são desdobramentos hiperfinos de um nível de estrutura fina, no qual está calculado a taxa de emissão espontênea ${ }^{2}$. Ao tomar o valor médio do sistema anterior e fazendo a mudança de variáveis

$$
\begin{aligned}
\rho_{i i} & \longrightarrow \rho_{i i}, \\
\rho_{12} & \longrightarrow e^{-i\left(\omega_{1} t-\phi_{2}\right)} \rho_{12}, \\
\rho_{32} & \longrightarrow e^{-i\left(\omega_{2} t-\phi_{1}\right)} \rho_{32}, \\
\rho_{13} & \longrightarrow e^{i\left(\omega_{2} t-\phi_{2}\right)} e^{-i\left(\omega_{1} t-\phi_{1}\right)} \rho_{13},
\end{aligned}
$$

chegamos ao conjunto de equações de Bloch já independentes do tempo,

$$
\begin{aligned}
& \dot{\rho}_{11}=-i\left|\Omega_{1}\right|\left(\rho_{21}-\rho_{12}\right)+\Gamma \rho_{22}, \\
& \dot{\rho}_{22}=i\left|\Omega_{1}\right| \rho_{21}+i\left|\Omega_{2}\right| \rho_{23}-\Gamma \rho_{22}+c . c . \\
& \dot{\rho}_{33}=-i\left|\Omega_{2}\right|\left(\rho_{23}-\rho_{32}\right)+\Gamma \rho_{22}, \\
& \dot{\rho}_{12}=-i \delta_{1} \rho_{12}-i\left|\Omega_{1}\right|\left(\rho_{22}-\rho_{11}\right)+i\left|\Omega_{2}\right| \rho_{13}-\Gamma \rho_{12}, \\
& \dot{\rho}_{32}=-i \delta_{2} \rho_{32}-i\left|\Omega_{2}\right|\left(\rho_{22}-\rho_{33}\right)+i\left|\Omega_{2}\right| \rho_{31}-\Gamma \rho_{32}, \\
& \dot{\rho}_{13}=-i \delta_{R} \rho_{13}+i\left|\Omega_{2}\right| \rho_{12}-i\left|\Omega_{1}\right| \rho_{23}-\gamma \rho_{13}, \\
& \dot{\rho}_{21}=\dot{\rho}_{12}^{*} \\
& \dot{\rho}_{23}=\dot{\rho}_{32}^{*} \\
& \dot{\rho}_{31}=\dot{\rho}_{13}^{*}
\end{aligned}
$$

onde $\delta_{R}=\delta_{1}-\delta_{2}$ é a dessintonia Raman.

Como visto no capítulo anterior, o sistema acima possui um vínculo. Para eliminá-lo, definimos as variáveis $w_{i}=\rho_{22}-\rho_{i i}$, que representam a diferença de população entre o estado excitado e os fundamentais. Para estas variáveis, podemos escrever o sistema

\footnotetext{
${ }^{2}$ No caso do Rubídio, esta é calculada para a linha $D 2$.
} 
anterior na notação matricial,

$$
\frac{d \mathbf{X}}{d t}=\mathbf{A X}+\mathbf{B}
$$

onde $\mathbf{X}=\left(w_{1}, w_{3}, \rho_{12}, \rho_{21}, \rho_{32}, \rho_{23}, \rho_{13}, \rho_{31}\right)^{T}, \mathbf{B}=\left(-\frac{2 \Gamma}{3},-\frac{2 \Gamma}{3}, 0,0,0,0,0,0\right)^{T} \mathrm{e}$

$$
\mathbf{A}=\left(\begin{array}{ccccc}
-2 \Gamma / 3 & -2 \Gamma / 3 & -2 i\left|\Omega_{1}\right| & 2 i\left|\Omega_{1}\right| & -i\left|\Omega_{2}\right| \\
-2 \Gamma / 3 & -2 \Gamma / 3 & -i\left|\Omega_{1}\right| & i\left|\Omega_{1}\right| & -2 i\left|\Omega_{2}\right| \\
-i\left|\Omega_{1}\right| & 0 & -\left(i \delta_{1}+\Gamma\right) & 0 & 0 \\
i\left|\Omega_{1}\right| & 0 & 0 & \left(i \delta_{1}-\Gamma\right) & 0 \\
0 & -i\left|\Omega_{2}\right| & 0 & 0 & -\left(i \delta_{2}+\Gamma\right) \\
0 & i\left|\Omega_{2}\right| & 0 & 0 & 0 \\
0 & 0 & i\left|\Omega_{2}\right| & 0 & 0 \\
0 & 0 & 0 & -i\left|\Omega_{2}\right| & i\left|\Omega_{1}\right|
\end{array}\right.
$$

$\left.\begin{array}{ccc}i\left|\Omega_{2}\right| & 0 & 0 \\ 2 i\left|\Omega_{2}\right| & 0 & 0 \\ 0 & i\left|\Omega_{2}\right| & 0 \\ 0 & 0 & -i\left|\Omega_{2}\right| \\ 0 & 0 & i\left|\Omega_{2}\right| \\ \left(i \delta_{2}-\Gamma\right) & -i\left|\Omega_{2}\right| & 0 \\ -i\left|\Omega_{1}\right| & -\left(i \delta_{R}+\gamma\right) & 0 \\ 0 & 0 & \left(i \delta_{R}-\gamma\right)\end{array}\right)$.

Resolvemos este sistema usando o Maple. Os gráficos do coeficiente de absorção, do índice de refração e das populações são mostrados na figura 4.4 para $\Omega_{1}=\Omega_{2}=\Gamma / 4$ e $\gamma=\Gamma / 100$. A dessintonia também está em unidades de $\Gamma$. Podemos ver que no sinal de absorção há uma queda para dessintonia nula. Este pico invertido, chamado de pico de EIT, é muito mais fino que o pico de absorção, cuja largura é $\Delta \omega=\Gamma \sqrt{3}$ neste caso. O fato da largura do pico ser muito mais fina que a largura natural, caracteriza um processo de interferência. A absorção e a população do estado excitado iriam a zero na ressonância se $\gamma=0$. Esta taxa de perda de coerência fenomenológica entre os estados fundamentais, fornece de absorção residual que pode ser obtida em ressonância.

O índice de refração possui um máximo na metade do pico de EIT. Essa alta nãolinearidade do meio, com baixa absorção é uma das responsáveis pelo grande potêncial da EIT de gerar estados não-clássicos.

No próximo capítulo, trataremos o átomo como um sistema de dois níveis com degenerescências. Este é o sistema onde fizemos nossas medidas. Todo o tratamento descrito 


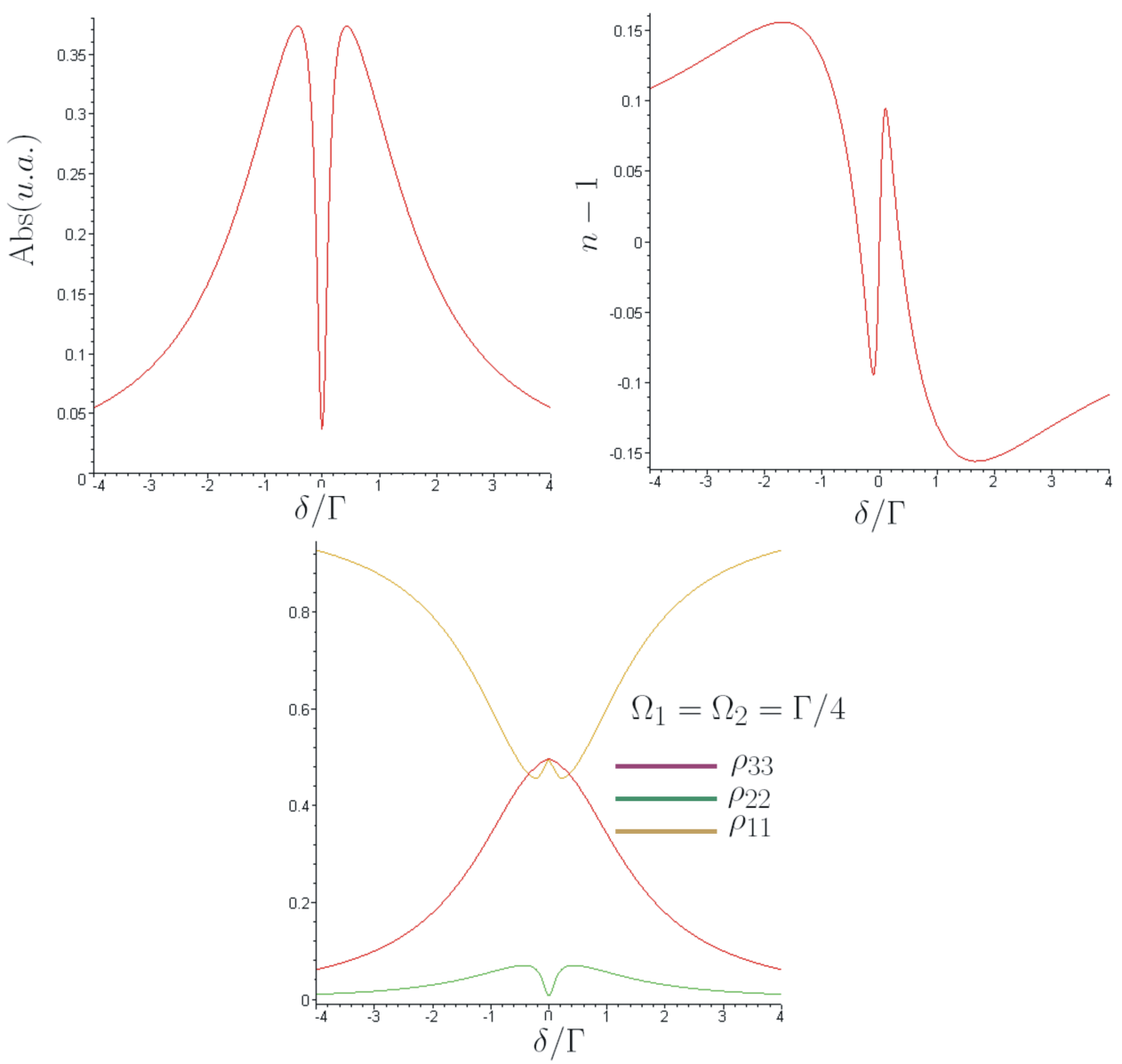

Figura 4.4: Absorção, indice de refração e população dos estados em função da dessintonia.

no capítulo 3 será generalizado para tratar este sistema. 


\section{$5 \quad$ Sistema de 2 Niveis com Degenerescências}

Neste capítulo trataremos do sistema de dois níveis em que tanto o estado excitado quando o fundamental são degenerados em energia. Como utilizamos em nossas medidas os estados hiperfinos do átomo de Rubídio, este é precisamente a configuração de níveis com a qual estamos tratando. Em muitas aplicações em que não haja fatores que afetem a degenerescência, como a presença de um campo magnético externo, o problema pode ser tratado como um sistema de dois níveis simples. Um grande exemplo em que a degenerescência dos níveis hiperfinos é de vital importância ocorre na teoria da armadilha magneto-ótica. Em que a teoria inicial, tratando o sistema como um átomo de dois níveis, previa uma temperatura limite para o resfriamento bem maior do que a observada experimentalmente [51]. E previsões corretas para a temperatuda foram obtidas considerado a degenerescência dos níveis hiperfinos [57].

Outro exemplo é o próprio efeito Hanle tratado neste capítulo. Em que a quebra de degenerescência devida ao campo magnético faz com que os átomos absorvam mais luz em uma componente de polarização circular que na outra. O que resulta na rotação da polarização linear ao passar por uma amostra atômica posicionada em um região com campo magnético não-nulo. Em nosso laboratório foram feitas diversas medidas acerca desse tipo de sistema juntamente com o grupo do prof. Arturo Lezama[19, 20]. Nestes trabalhos foram apresentadas medidas das flutuações do campo eletromagnético ao passar por uma amostra atômica que estava na presença de um campo magnético. Também foram apresentados modelos teóricos para os sinais de ruído medidos utilizando campos clássicos com fase estocástica [25].

O tratamento feito neste capítulo generaliza os conceitos introduzidos no capítulo 3 para o sistema de dois níveis com degenerescências interagindo com um campo magnético. Utilizamos a quantização do campo eletromagnético para gerar um modelo para o ruído, como em [21]. 


\subsection{Hamiltoniano da Interação Átomo-Luz}

A interação com o campo eletromagnético será feita como no capítulo 3, onde o átomo interage com todos os modos do campo como na figura 5.1. No caso em que $F_{a}>F_{b}$, este diagrama de níveis é formado por vários sistemas- $\Lambda$ acoplados. Porém, diferentemente do capítulo anterior, manteremos o laser em ressonância e mudaremos os níveis de energia por meio da varredura do campo magnético externo. Em princípio, ambas as formas de varredura devem ser equivalentes e dar origem ao fenômeno da EIT. Neste caso as componentes $\sigma^{ \pm}$do campo incidente fazem o papel de feixes de prova e de bombeio e o fenômeno é chamado de Hanle-EIT. Para $F_{a}<F_{b}$, o sistema pode ser visto como um conjunto de sistemas-N acoplados[25], o podemos observar o fenômeno de Hanle-EIA. Para $F_{a}=F_{b}$, também observaremos Hanle-EIT, pois a quantidade se sistemas- $\Lambda$ é maior que de sistemas-N.

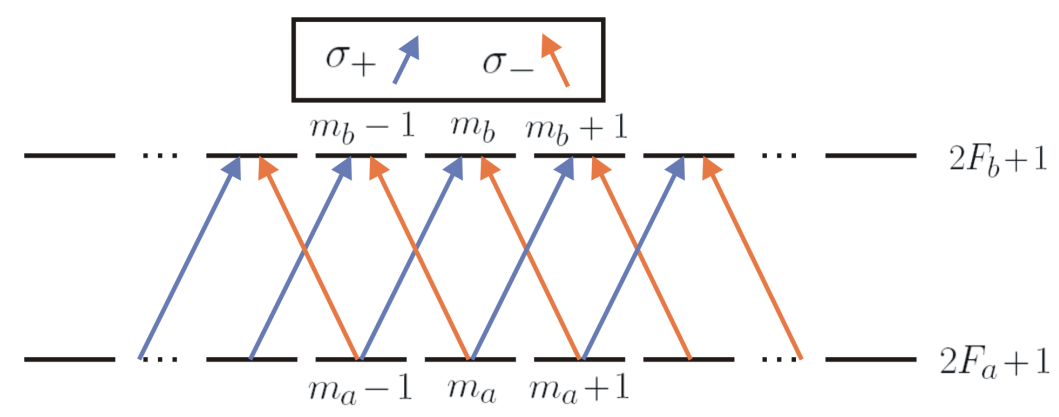

Figura 5.1: Diagrama de niveis para a interação somente com o feixe incedente.

Os estados fundamentais e excitados serão indexados por $\left|m_{a}\right\rangle=\left|E_{a} j_{a} m_{a}\right\rangle$ e $\left|m_{b}\right\rangle=$ $\left|E_{b} j_{b} m_{b}\right\rangle$ respectivamente. Como de costume, $E_{i}$ representa a energia no nível hiperfino, $j_{i}$ o momento angular total e $m_{i}$ a componente do mesmo na direção $z$. O hamiltoniano do átomo livre será

$$
H_{0}=\hbar \omega_{b} \hat{P}_{b}+\hbar \omega_{a} \hat{P}_{a}
$$

onde $\hat{P}_{b}=\sum_{m_{b}} \hat{\sigma}_{m_{b} m_{b}}$ e $\hat{P}_{a}=\sum_{m_{a}} \hat{\sigma}_{m_{a} m_{a}}$, tal que $\hat{P}_{a}+\hat{P}_{b}=1$. O hamiltoniano do campo livre, assim como o hamiltoniano de interação, são os mesmos que no capítulo 3. Expandimos o operador de dipolo na base atômica como

$$
\hat{\mathbf{d}}=\hat{P}_{a} \hat{\mathbf{d}} \hat{P}_{a}+\hat{P}_{a} \hat{\mathbf{d}} \hat{P}_{b}+\hat{P}_{b} \hat{\mathbf{d}} \hat{P}_{a}+\hat{P}_{b} \hat{\mathbf{d}} \hat{P}_{b}
$$

Como $\hat{\mathbf{d}}$ é impar por inversão espacial e os estados $\left|m_{b}\right\rangle$ e $\left|m_{a}\right\rangle$ podem ser escolhidos de modo que sua paridade seja definida e oposta, então $\hat{P}_{a} \hat{\mathbf{d}} \hat{P}_{a}=\hat{P}_{b} \hat{\mathbf{d}} \hat{P}_{b}=0$, portanto,

$$
V=\left(\hat{P}_{a} \hat{\mathbf{d}} \hat{P}_{b}+\hat{P}_{b} \hat{\mathbf{d}} \hat{P}_{a}\right) \cdot\left(\hat{\mathbf{E}}^{(+)}+\hat{\mathbf{E}}^{(-)}\right)
$$


Neste caso, a RWA consiste em desprezar os operadores $\hat{P}_{a} \hat{\mathbf{d}} \hat{P}_{b} \cdot \hat{\mathbf{E}}^{(+)}$e $\hat{P}_{b} \hat{\mathbf{d}} \hat{P}_{a} \cdot \hat{\mathbf{E}}^{(-)}$. Sendo assim,

$$
V_{R W A}=\hat{P}_{b} \hat{\mathbf{d}} \hat{P}_{a} \cdot \hat{\mathbf{E}}^{(+)}+\hat{P}_{a} \hat{\mathbf{d}} \hat{P}_{b} \cdot \hat{\mathbf{E}}^{(-)}
$$

\subsection{Efeito Hanle e Interação com o Campo Magnético}

Se incidimos um feixe linearmente polarizado em uma amostra atômica presente em um região na qual há um campo magnético não-nulo, ocorre uma rotação na polarização do campo transmitido em relação à do campo incidente, cujo sentido depende do sentido do campo magnético. Este fenômeno é conhecido como efeito Hanle [40] e ocorre devido á absorção preferencial de uma das componentes da polarização na base circular.
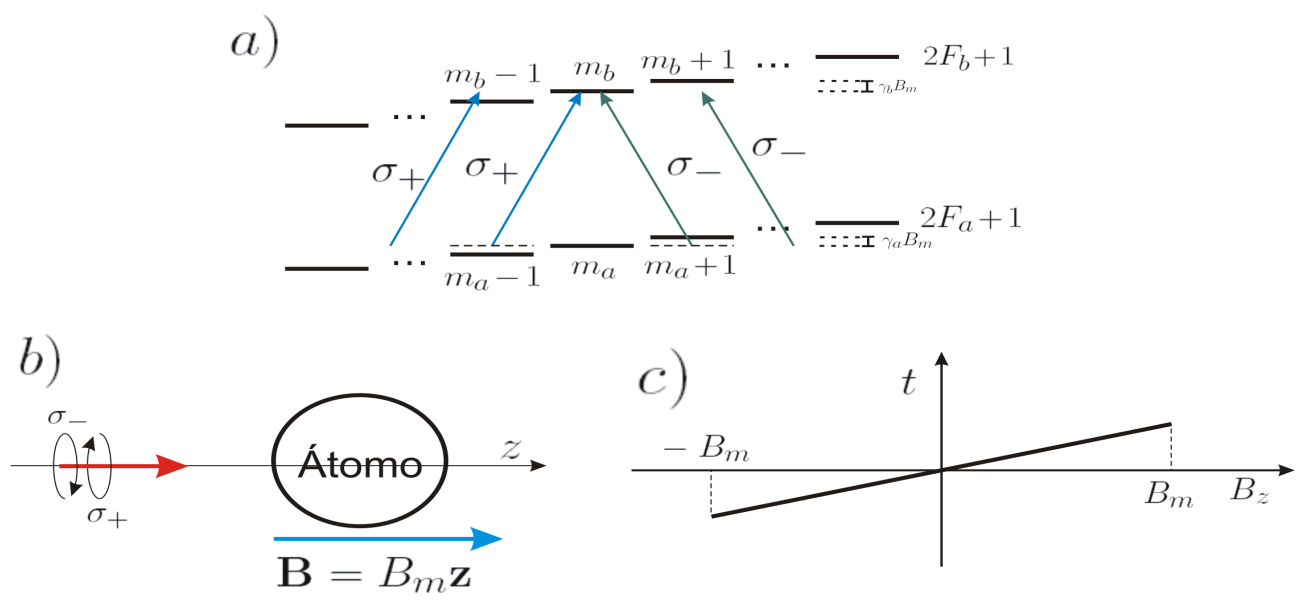

Figura 5.2: a) Diagrama de níveis após interação com o campo magnético. b) Interação dos átomos com com a luz e o campo magnético. c) Varredura campo magnético no tempo

A interação de um átomo com um campo magnético no regime de baixas energias será dado pelo hamiltoniano de dipolo magnético [39, 28, 27],

$$
\begin{aligned}
H_{m} & =\hat{\mu} \cdot \mathbf{B} \\
\hat{\mu} & =\frac{\mu_{B}}{\hbar}\left[\hat{\mathbf{L}}+g_{e} \sum_{i} \hat{\mathbf{S}}_{e}^{i}-\frac{m_{e}}{m_{N}} g_{N} \hat{\mathbf{S}}_{N}\right], \\
\mu_{B} & =\frac{e \hbar}{2 m_{e} c}
\end{aligned}
$$

onde $g_{e}$ e $g_{N}$ são as constantes giromagnéticas do elétron de do núcleo respectivamente, $\mu_{B}$ é o magnetón de Bohr, $\hat{\mathbf{L}}$ o momento angular orbital, $\hat{\mathbf{S}}_{e}^{i}$ o spin de cada elétron e $\hat{\mathbf{S}}_{N}$ é o spin do núcleo. Sendo $\hat{\mathbf{J}}=\hat{\mathbf{L}}+\sum_{i} \hat{\mathbf{S}}_{e}^{i}+\hat{\mathbf{S}}_{N}$ o momento angular total, em cuja componente $z$ os estados de estrutura hiperfina $\left|m_{a}\right\rangle$ e $\left|m_{b}\right\rangle$ são diagonais. $m_{e}$ e $m_{N}$ são 
as massas no elétron de no núcleo, tal que $m_{e} \ll m_{N}$. Podemos introduzir a relação de fechamento na expressão para $H_{m}$ e obter,

$$
H_{m}=\left[P_{a} \hat{\mu} P_{a}+P_{b} \hat{\mu} P_{b}\right] \cdot \mathbf{B},
$$

pois, como $\hat{\mu}$ é par por reversão espacial, $P_{a} \mu P_{b}=0$. Todas as médias na expressão acima são feitas no espaço de $j$ fixo, então podemos usar a relação (??) e escrever todos os spins e o $\hat{\mathbf{L}}$ como proporcionais a $\hat{\mathbf{J}}$, também alinharemos o eixo $z$ com o campo magnético. Isso faz com que o hamiltoniano se torne

$$
H_{m}=\left(\gamma_{a} P_{a}+\gamma_{b} P_{b}\right) B \hat{J}_{z}
$$

onde $\gamma_{i}=\mu_{B} g_{i} / \hbar$, com $g_{i}$ sendo uma constante giromagnética efetiva resultado da soma em (5.3).

\subsection{Equações de Heisenberg-Langevin}

Seguindo a mesma linha do capítulo 3, trataremos o sistema usando as equações de Heisenberg-Langevin. O campo magnético que separa os subníveis Zeeman será tratado classicamente, pois oscila em baixa frequência. Para $\hat{a}_{\mathbf{k} \lambda}$ e $\hat{\sigma}_{i j}$ obtemos, como em (3.10) e $(3.11)$,

$$
\begin{aligned}
\frac{d \hat{a}_{\mathbf{k} \lambda}(t)}{d t}= & -i \omega \hat{a}_{\mathbf{k} \lambda}(t)-\sqrt{\frac{\omega}{2 V \hbar}} \sum_{m_{a} m_{b}}\left(\hat{e}_{\mathbf{k} \lambda} \cdot \mathbf{d}_{m_{a} m_{b}}\right) \hat{\sigma}_{m_{a} m_{b}}(t), \\
\frac{d \hat{\sigma}_{i j}(t)}{d t}= & i \omega_{i j} \hat{\sigma}_{i j}(t)+\sum_{m_{a}, m_{b}}\left\{\frac{\mathbf{d}_{m_{a} m_{b}} \cdot \hat{\mathbf{E}}^{(-)}(t)}{i \hbar}\left[\delta_{j m_{a}} \hat{\sigma}_{i m_{b}}(t)-\delta_{i m_{b}} \hat{\sigma}_{m_{a} j}(t)\right]\right. \\
& \left.+\frac{\mathbf{d}_{m_{b} m_{a}} \cdot \hat{\mathbf{E}}^{(+)}(t)}{i \hbar}\left[\delta_{j m_{b}} \hat{\sigma}_{i m_{a}}(t)-\delta_{i m_{a}} \hat{\sigma}_{m_{b} j}(t)\right]\right\}
\end{aligned}
$$

portanto, (5.4) fica

$$
\hat{a}_{\mathbf{k} \lambda}(t)=e^{-i \omega t} \hat{a}_{\mathbf{k} \lambda}-\sqrt{\frac{\omega}{2 V \hbar}} \sum_{m_{a} m_{b}}\left(\hat{e}_{\mathbf{k} \lambda}^{*} \cdot \mathbf{d}_{m_{a} m_{b}}\right) \int_{0}^{t} e^{-i \omega\left(t-t^{\prime}\right)} \hat{\sigma}_{m_{a} m_{b}}\left(t^{\prime}\right) d t^{\prime} .
$$


Realizando o mesmo cálculo feito no capítulo anterior para cada $\mathbf{d}_{m_{a} m_{b}}$, podemos mostrar que

$$
\begin{aligned}
\hat{\mathbf{E}}_{l}^{(+)}(t) & =i \sum_{\mathbf{k} \lambda} \sqrt{\frac{2 \pi \hbar \omega}{V}} \hat{a}_{\mathbf{k} \lambda} \mathbf{e}_{\mathbf{k} \lambda} e^{-i \omega t}, \\
\hat{\mathbf{E}}_{p}^{(+)}(t) & =-\frac{2 i \omega_{0}^{3}}{3 c^{3}} \sum_{m_{a} m_{b}} \mathbf{d}_{m_{a} m_{b}} \hat{\sigma}_{m_{a} m_{b}}(t) .
\end{aligned}
$$

Esse resultado nos mostra que o campo de polarização na posição do átomo depende de todas as transições possíveis de todos os estados excitados para todos os estados fundamentais. Como podemos mostrar pelo Teorema de Wigner-Eckart, o fato de $\hat{\mathbf{d}}$ ser um operador vetorial garante que apenas transições com $m_{b}-m_{a}=0, \pm 1$ sejam possíveis, as quais correspondem às componentes $\pi, \sigma^{ \pm}$do campo eletromagnético. Como as outras transições se anulam identicamente, não há problema em manter a soma sobre as transições proibidas. Para futura referência, reescreveremos (5.5) de forma mais simplificada,

$$
\begin{aligned}
\frac{d \hat{\sigma}_{i j}(t)}{d t}= & -i \omega_{i j} \hat{\sigma}_{i j}(t)+\frac{1}{i \hbar} \sum_{m_{a}}\left[\mathbf{d}_{j m_{a}} \cdot \hat{\mathbf{E}}^{(+)}(t) \hat{\sigma}_{i m_{a}}(t)-\mathbf{d}_{m_{a} i} \cdot \hat{\mathbf{E}}^{(-)}(t) \hat{\sigma}_{m_{a} j}(t)\right] \\
& -\frac{1}{i \hbar} \sum_{m_{b}}\left[\mathbf{d}_{m_{b} i} \cdot \hat{\mathbf{E}}^{(+)}(t) \hat{\sigma}_{m_{b} j}(t)-\mathbf{d}_{j m_{b}} \cdot \hat{\mathbf{E}}^{(-)}(t) \hat{\sigma}_{i m_{b}}(t)\right]
\end{aligned}
$$

Para chegar às equações de Heisenberg-Langevin, precisamos subistituir $\hat{\mathbf{E}}_{l}^{(+)}(t)$ e $\hat{\mathbf{E}}_{p}^{(+)}(t)$ em (5.8). Pelo fato de a quantidade de equações aqui ser muito maior que nos casos anteriores, analisaremos o problema de forma menos direta começando pela substituição do campo de polarização e o cálculo das contribuições dissipativas.

\subsection{Contribuições Dissipativas}

Ao substituir (5.7) em (5.8), obteremos os termos dissipativos, $\left.\dot{\hat{\sigma}}_{i j}(t)\right|^{\text {dis }}$. Para uma melhor compreensão, analisaremos de forma separada os subespaços $b b, a a, a b$ e $b a$ mostrados na figura 5.3. Definimos a ordem normal agora como

$$
\hat{a}_{\mathbf{k} \lambda}^{\dagger}, \hat{\sigma}_{b a}(t), \hat{\sigma}_{D D}(t), \hat{\sigma}_{a b}(t), \hat{a}_{\mathbf{k} \lambda}
$$

onde $D=a$ ou $b$.

O símbolo $\hat{\sigma}_{b a}$ representa o conjunto de todos $\hat{\sigma}_{i j}^{\prime} s$, pertencentes ao subespaço $b a$. Note que a ordenação dentro deste subespaço é arbitrária, pois $\left[\hat{\sigma}_{m_{b} m_{a}}, \hat{\sigma}_{m_{b}^{\prime} m_{a}^{\prime}}\right]=0$. Sendo que o mesmo vale para o subespaço $a b$. Da mesma forma, $D D$ representa os subespaços 


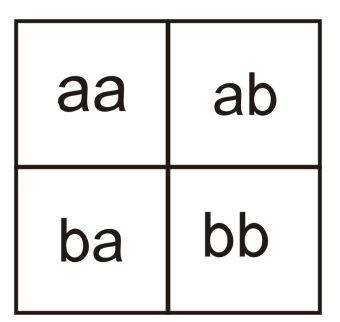

Figura 5.3: Diagrama da matriz formada pelos $\hat{\sigma}_{i j}$ separando os subespaços.

diagonais. E como $\left[\hat{\sigma}_{m_{b} m_{b}^{\prime}}, \hat{\sigma}_{m_{a} m_{a}^{\prime}}\right]=0$, não haverá problemas de ordenação entre $\hat{\sigma}_{i j}^{\prime} s$ pertencentes subespaços diagonais diferentes. Porém, dentro de cada subespaço diagonal, teremos que definir uma ordem na qual fazer a substituição, pois $\left[\hat{\sigma}_{m_{a} m_{a}^{\prime}}, \hat{\sigma}_{m_{a}^{\prime \prime} m_{a}^{\prime \prime \prime}}\right] \neq 0$, tal que o mesmo vale para o subespaço $b b$. Esta ordenação, entretanto, se mostrará irrelevante devido ao fato de que $\hat{\mathbf{E}}_{p}^{(+)}(t)$ em (5.7) está definido apenas no subespaço $a b$.

Para o subespaço bb, $\mathbf{d}_{m_{b} i}=\mathbf{d}_{j m_{b}}=0$, então, já substituindo $\hat{\mathbf{E}}_{p}^{( \pm)}(t)$ em (5.8) na ordem normal, podemos escrever

$$
\left.\dot{\hat{\sigma}}_{m_{b} m_{b}^{\prime}}\right|^{d i s}(t)=-\frac{\omega_{0}^{3}}{6 \pi \hbar c^{3}} \sum_{m_{a}, m_{b}^{\prime \prime}}\left[\mathbf{d}_{m_{b}^{\prime} m_{a}} \cdot \mathbf{d}_{m_{a} m_{b}^{\prime \prime}} \hat{\sigma}_{m_{b} m_{b}^{\prime \prime}}(t)+\mathbf{d}_{m_{a} m_{b}} \cdot \mathbf{d}_{m_{b}^{\prime \prime} m_{a}} \hat{\sigma}_{m_{b}^{\prime \prime} m_{b}^{\prime}}(t)\right] .
$$

Expandindo $\mathbf{d}$ na base esférica,

$$
\hat{\mathbf{d}}=\sum_{q=-1,0,1} \hat{d}_{q} \mathbf{e}_{q}=\sum_{q=-1,0,1} \hat{d}_{q}^{\dagger} \mathbf{e}_{q}^{*}
$$

$\operatorname{com} \mathbf{e}_{q} \cdot \mathbf{e}_{q^{\prime}}=\delta_{q q^{\prime}}$, portanto,

$$
\hat{\mathbf{d}}_{m_{b}^{\prime} m_{a}} \cdot \mathbf{d}_{m_{a} m_{b}^{\prime \prime}}=\sum_{q=-1,0,1}\left\langle m_{b}^{\prime}\left|d_{q}\right| m_{a}\right\rangle\left\langle m_{a}\left|\hat{d}_{q}^{\dagger}\right| m_{b}^{\prime \prime}\right\rangle
$$

Como visto em (2.33), e pelo fato de $\left\langle m_{a}\left|\hat{d}_{q}^{\dagger}\right| m_{b}^{\prime \prime}\right\rangle=\left\langle m_{b}^{\prime \prime}\left|\hat{d}_{q}\right| m_{a}\right\rangle^{*}$

$$
\begin{aligned}
\left\langle m_{b}^{\prime}\left|\hat{d}_{q}\right| m_{a}\right\rangle & =\frac{1}{\sqrt{2 j_{b}+1}}\left\langle E_{b} j_{b}\|\hat{d}\| E_{a} j_{a}\right\rangle\left\langle j_{b} m_{b}^{\prime} \mid j_{a} 1 m_{a} q\right\rangle, \\
\left\langle m_{a}\left|\hat{d}_{q}^{\dagger}\right| m_{b}^{\prime \prime}\right\rangle & =\frac{1}{\sqrt{2 j_{b}+1}}\left\langle E_{b} j_{b}\|\hat{d}\| E_{a} j_{a}\right\rangle^{*}\left\langle j_{a} 1 m_{a} q \mid j_{b} m_{b}^{\prime \prime}\right\rangle .
\end{aligned}
$$

Realizando a soma em $m_{a}$ e usando a relação de fechamento, chegamos á expressão

$$
\sum_{m_{a}} \mathbf{d}_{m_{b}^{\prime} m_{a}} \cdot \mathbf{d}_{m_{a} m_{b}^{\prime \prime}}=\frac{1}{2 j_{b}+1}\left|\left\langle E_{b} j_{b}\|\hat{d}\| E_{a} j_{a}\right\rangle\right|^{2} \delta_{m_{b}^{\prime}, m_{b}^{\prime \prime}}
$$


Ao substituir esse resultado em (5.9) obtemos

$$
\begin{aligned}
\left.\dot{\hat{\sigma}}_{m_{b} m_{b}^{\prime}}(t)\right|^{d i s} & =-\Gamma \hat{\sigma}_{m_{b} m_{b}^{\prime}}(t), \\
\Gamma & =\frac{\omega_{0}^{3}}{3 \pi \hbar c^{3}\left(2 j_{b}+1\right)}\left|\left\langle E_{b} j_{b}\|\hat{d}\| E_{a} j_{a}\right\rangle\right|^{2}
\end{aligned}
$$

Para $a b, \mathbf{d}_{j m_{b}}=\mathbf{d}_{m_{a} i}=0$, então

$$
\left.\dot{\hat{\sigma}}_{m_{a} m_{b}}(t)\right|^{d i s}=-\frac{\omega_{0}^{3}}{6 \pi \hbar c^{3}} \sum_{m_{a}^{\prime}, m_{b}^{\prime \prime}} \mathbf{d}_{m_{b} m_{a}^{\prime}} \cdot \mathbf{d}_{m_{a}^{\prime} m_{b}^{\prime \prime}} \hat{\sigma}_{m_{a} m_{b}^{\prime \prime}}(t)
$$

portanto, usando (5.13) e com $\Gamma$ definido em (5.15),

$$
\left.\dot{\hat{\sigma}}_{m_{a} m_{b}}(t)\right|^{d i s}=-\frac{\Gamma}{2} \hat{\sigma}_{m_{a} m_{b}}(t) .
$$

Como os $\hat{\sigma}_{i j}^{\dagger}=\hat{\sigma}_{j i}$, então cada elemento de $a b$, terá seu conjugado hermitiano em $b a$. Desta forma, os coeficientes dos termos dissipativos será o mesmo que em (5.16)

Para $a a, \mathbf{d}_{j m_{a}}=\mathbf{d}_{m_{a} i}=0$, portanto,

$$
\left.\dot{\hat{\sigma}}_{m_{a} m_{a}^{\prime}}\right|^{d i s}(t)=\frac{\omega_{0}^{3}}{6 \pi \hbar c^{3}} \sum_{m_{b}, m_{b}^{\prime \prime}}\left[\mathbf{d}_{m_{b} m_{a}} \cdot \mathbf{d}_{m_{a}^{\prime} m_{b}^{\prime \prime}} \hat{\sigma}_{m_{b} m_{b}^{\prime \prime}}(t)+\mathbf{d}_{m_{a}^{\prime} m_{b}} \cdot \mathbf{d}_{m_{b}^{\prime \prime} m_{a}} \hat{\sigma}_{m_{b}^{\prime \prime} m_{b}}(t)\right] .
$$

Realizando o mesmo cálculo anterior podemos mostrar que

$$
\left.\dot{\hat{\sigma}}_{m_{a} m_{a}^{\prime}}\right|^{d i s}(t)=\Gamma \sum_{q, m_{b}, m_{b}^{\prime \prime}}\left\langle j_{b} m_{b} \mid j_{a} 1 m_{a} q\right\rangle\left\langle j_{a} 1 m_{a}^{\prime} q \mid j_{b} m_{b}^{\prime \prime}\right\rangle \hat{\sigma}_{m_{b} m_{b}^{\prime \prime}}(t) .
$$

Para podermos escrever de forma mais simplificada os termos dissipativos, definimos o operador de dipolo reduzido como

$$
\hat{\mathbf{Q}}=\frac{\hat{\mathbf{d}}}{\left\langle E_{b} j_{b}\|\hat{d}\| E_{a} j_{a}\right\rangle} .
$$

Por meio de (5.11) e (5.12), podemos excrever $\left.\dot{\hat{\sigma}}_{m_{a} m_{a}^{\prime}}\right|^{d i s}(t)$ como

$$
\left.\dot{\hat{\sigma}}_{m_{a} m_{a}^{\prime}}\right|^{d i s}(t)=\left(2 j_{b}+1\right) \Gamma \sum_{q, m_{b}, m_{b}^{\prime \prime}}\left\langle m_{b}\left|\hat{Q}_{q}\right| m_{a}\right\rangle\left\langle m_{a}^{\prime}\left|\hat{Q}_{q}^{\dagger}\right| m_{b}^{\prime \prime}\right\rangle \hat{\sigma}_{m_{b} m_{b}^{\prime \prime}}(t)
$$

Tomaremos a média dester resultados para obter as contribuições dos termos dissipativos para as equações de Bloch do sistema. Para (5.19) temos

$$
\left.\dot{\rho}_{m_{a} m_{a}^{\prime}}\right|^{d i s}(t)=\left(2 j_{b}+1\right) \Gamma \sum_{q, m_{b}, m_{b}^{\prime \prime}}\left\langle m_{b}\left|\hat{Q}_{q}\right| m_{a}^{\prime}\right\rangle\left\langle m_{a}\left|\hat{Q}_{q}^{\dagger}\right| m_{b}^{\prime \prime}\right\rangle \rho_{m_{b}^{\prime \prime} m_{b}}(t),
$$


o que, junto com (5.14) e (5.16), nos leva a

$$
\begin{aligned}
\left.\dot{\rho}_{m_{a} m_{a}^{\prime}}\right|^{d i s}(t) & =\left(2 j_{b}+1\right) \Gamma \sum_{q=-1,0,1}\left\langle m_{a}\left|\hat{Q}_{q}^{\dagger} P_{b} \rho(t) P_{b} \hat{Q}_{q}\right| m_{a}^{\prime}\right\rangle \\
\left.\dot{\rho}_{m_{b} m_{b}^{\prime}}\right|^{d i s}(t) & =-\Gamma \rho_{m_{b} m_{b}^{\prime}}(t), \\
\left.\dot{\rho}_{m_{a} m_{b}}\right|^{d i s}(t) & =-\frac{\Gamma}{2} \rho_{m_{a} m_{b}}(t), .
\end{aligned}
$$

Na relação (5.20), podemos tomar $m_{a}=m_{a}^{\prime}$, o que fornecerá a informação sobre como a dissipação afeta as populações do estado fundamental. Pela regra de seleção, $m_{b}=m_{a}+q$ e $m_{b}^{\prime \prime}=m_{a}+q$, portanto, apenas os termos diagonais fornecem contribuições não-nulas para $\left.\dot{\rho}_{m_{a} m_{a}}\right|^{d i s}(t)$. Sendo assim,

$$
\left.\dot{\rho}_{m_{a} m_{a}}\right|^{d i s}(t)=\left(2 j_{b}+1\right) \Gamma \sum_{q, m_{b}}\left|\left\langle m_{b}\left|\hat{Q}_{q}\right| m_{a}\right\rangle\right|^{2} \rho_{m_{b} m_{b}}(t)
$$

Como pode ser visto em (5.21), não há troca de coerência entre os estados excitados nem influência dos estados fundamentais nos mesmos. Todo o bloco $b b$ perde população e coerência a uma mesma taxa, a qual possui a mesma forma que para o sistema de dois níveis dividida pela quantidade de estados excitados e com elemento de matriz raduzido fazendo o papel do elemento de matriz. Naquele caso, o bloco possuía apenas um componente, que era a população do estado excitado, então não havia sentido falar em troca ou não de coerência. Para o bloco $a b$ e $b a$, o termo dissipativo também possui a mesma forma que no caso sem degenerescências. A presença de mais de um estado excitado e fundamental não afeta a coerência dentro destes subespaços. Desta forma, nestes três subespaços o sistema tratado é equivalente ao sistema de dois níveis. Em princípio, não seria de se esperar esse comportamento, pois o processo de emissão de luz por $\left|m_{b}\right\rangle$ e absorção por $\left|m_{b}^{\prime}\right\rangle$ poderia levar a trocas de corência entre os estados excitados, ou mesmo entre estados excitados e fundamentais.

Como podemos extrair de (5.23) e (2.33), cada nível fundamental $\left|m_{a}\right\rangle$ recebe população à mesma taxa dos estados $\left|m_{b}+q\right\rangle$, onde $q=0, \pm 1$, como ser visto na 5.4. Da expressão (5.20) podemos ver que os estados fundamentais recebem coerência dos estados excitados. Esta ganho de coerência, que no sistema de três níveis é representada pela taxa $\gamma$, faz com que ainda haja uma pequena absorção mesmo em ressonância. Note que diferentemente do sistema de três níveis, no qual a mesmo aparece como um termo fenômenológico, aqui ela possui origem radiativa. Esta coerência entre os estados fundamentais é fundamental para o fenômeno da EIA, que também pode ser observada nesse tipo de sistema e é tratada com detalhes em [24, 25]. 


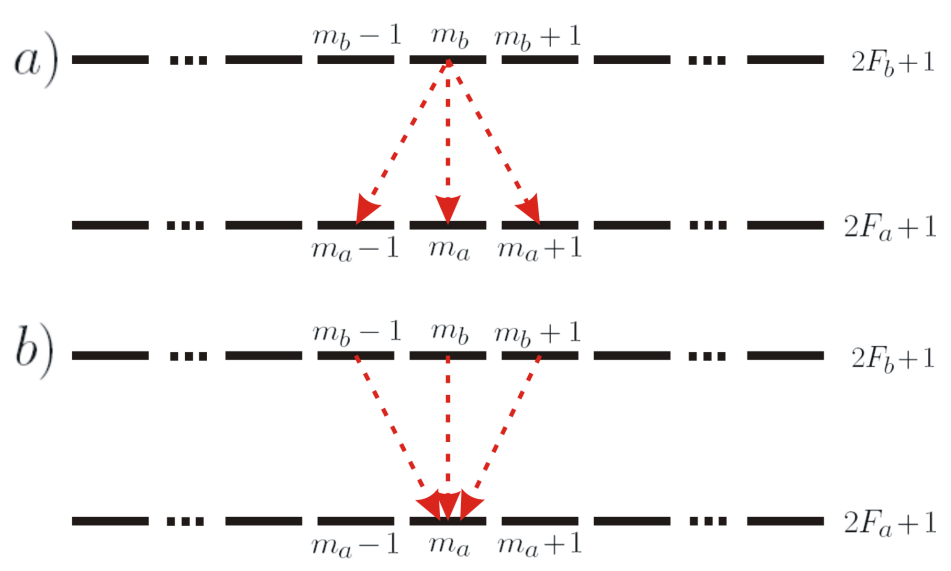

Figura 5.4: a) População perdida pelo estado excitado $\left|m_{b}\right\rangle$. b) População recebida pelo estado fundamental $\left|m_{a}\right\rangle$.

Podemos adicionar fenomenologicamente mais um termo de decaimento não radiativo, que seria equivalente a $\gamma$. Físicamente, este termo considera as perdas pelo tamanho finito do feixe laser. Ele é um balanço entre os átomos que saem e os que entram multiplicado pela taxa de entrada e saída, $\kappa$, suposta a mesma para entrada e saída,

$$
\left.\dot{\rho}\right|^{\tilde{n} R a d}(t)=-\kappa\left(\rho(t)-\rho_{0}\right)
$$

tal que $\rho_{0}=P_{a} / \sqrt{2 j_{a}+1}$ está distribuida igualmente entre todos os estados fundamentais. Podemos escrever todas estas contribuições de forma mais compacta, como foi feito no capítulo 3, com $\hat{Q}_{a b}^{\dagger q} \equiv P_{a} \hat{Q}_{q}^{\dagger} P_{b}=\left(\hat{Q}_{b a}^{q}\right)^{\dagger}$,

$$
\dot{\rho}^{d i s}(t)=-\frac{\Gamma}{2}\left\{P_{b}, \rho(t)\right\}+\Gamma\left(2 j_{b}+1\right) \sum_{q=-1,0,1} \hat{Q}_{a b}^{\dagger q} \rho(t) \hat{Q}_{b a}^{q}-\kappa\left(\rho-\rho_{0}\right),
$$

onde o primeiro termo representa as contribuições (5.21) e (5.22), e o segundo termo a (5.20). Esta equação é similar a (3.48).

\subsection{Contribuição do Campo Incidente}

Vamos substituir $\hat{\mathbf{E}}_{l}^{(+)}(t)=\sum_{\mathbf{k} \lambda} \hat{\boldsymbol{\Xi}}_{\mathbf{k} \lambda} e^{i \omega t}$ nas equações (5.8). A definição de $\hat{\boldsymbol{\Xi}}_{\mathbf{k} \lambda}$ pode ser vista em (3.14) e a componente que representa a fonte laser é o campo incidente, $\hat{\mathbf{E}}_{i n}^{(+)} \equiv \hat{\Xi}_{\mathbf{k}_{L} \lambda_{L}}$. Prosseguimos com a substituição para calcular a contribuição do campo 
livre,

$$
\begin{aligned}
\left.\dot{\hat{\sigma}}_{i j}(t)\right|^{f}= & \frac{1}{i \hbar} \sum_{m_{a}}\left[\hat{\sigma}_{i m_{a}}(t) \mathbf{d}_{j m_{a}} \cdot \hat{\mathbf{E}}_{i n}^{(+)} e^{-i \omega_{L} t}-\mathbf{d}_{m_{a} i} \cdot \hat{\mathbf{E}}_{i n}^{(-)} e^{i \omega_{L} t} \hat{\sigma}_{m_{a} j}(t)\right]+\hat{F}_{i j}(t) \\
& -\frac{1}{i \hbar} \sum_{m_{b}}\left[\hat{\sigma}_{m_{b} j}(t) \mathbf{d}_{m_{b} i} \cdot \hat{\mathbf{E}}_{i n}^{(+)} e^{-i \omega_{L} t}-\mathbf{d}_{j m_{b}} \cdot \hat{\mathbf{E}}_{i n}^{(-)} e^{i \omega_{L} t} \hat{\sigma}_{i m_{b}}(t)\right]
\end{aligned}
$$

com as forças de Langevin sendo dadas por

$$
\begin{aligned}
\hat{F}_{i j}(t)= & \sum_{\mathbf{k} \neq \mathbf{k}_{L} \lambda \neq \lambda_{L}}\left\{\sum_{m_{a}} \frac{1}{i \hbar}\left[\hat{\sigma}_{i m_{a}}(t) \mathbf{d}_{j m_{a}} \cdot \hat{\boldsymbol{\Xi}}_{\mathbf{k} \lambda} e^{-i \omega t}-\mathbf{d}_{m_{a} i} \cdot \hat{\boldsymbol{\Xi}}_{\mathbf{k} \lambda}^{\dagger} e^{i \omega t} \hat{\sigma}_{m_{a} j}(t)\right]\right. \\
& \left.-\frac{1}{i \hbar} \sum_{m_{b}}\left[\hat{\sigma}_{m_{b} j}(t) \mathbf{d}_{m_{b} i} \cdot \hat{\boldsymbol{\Xi}}_{\mathbf{k} \lambda} e^{-i \omega t}-\mathbf{d}_{j m_{b}} \cdot \hat{\boldsymbol{\Xi}}_{\mathbf{k} \lambda}^{\dagger} e^{i \omega t} \hat{\sigma}_{i m_{b}}(t)\right]\right\} .
\end{aligned}
$$

Tomando a média de (5.25), chegamos à contribuição desse termo para as equações de Bloch, com $\mathbf{E}_{\text {in }}=\left\langle\hat{\mathbf{E}}_{\text {in }}^{(+)}\right\rangle$,

$$
\begin{aligned}
\left.i \hbar \dot{\rho}_{j i}(t)\right|^{f}= & \sum_{m_{a}}\left[\rho_{m_{a} i}(t) \mathbf{d}_{j m_{a}} \cdot \mathbf{E}_{i n} e^{-i \omega_{L} t}-\mathbf{d}_{m_{a} i} \cdot \mathbf{E}_{i n}^{*} e^{i \omega_{L} t} \rho_{j m_{a}}(t)\right] \\
& -\sum_{m_{b}}\left[\rho_{j m_{b}}(t) \mathbf{d}_{m_{b} i} \cdot \mathbf{E}_{i n} e^{-i \omega_{L} t}-\mathbf{d}_{j m_{b}} \cdot \mathbf{E}_{i n}^{*} e^{i \omega_{L} t} \rho_{m_{b} i}(t)\right]
\end{aligned}
$$

Podemos escrever então,

$$
\left.i \dot{\rho}(t)\right|^{f}=\left[\hat{\mathbf{g}} P_{a} \rho(t)-\rho(t) P_{b} \hat{\mathbf{g}}\right] e^{-i \omega_{L} t}+\left[\hat{\mathbf{g}}^{\dagger} P_{b} \rho(t)-\rho(t) P_{a} \hat{\mathbf{g}}^{\dagger}\right] e^{i \omega_{L} t},
$$

onde $\hat{\mathbf{g}}=\left(\hat{\mathbf{d}} \cdot \mathbf{E}_{i n}\right) / \hbar$ é um operador cuja média será a frequência de Rabi. Ao introduzir a relação de fechamento, $P_{a}+P_{b}=1$, em cada termo da equação anterior e como $P_{a} \hat{\mathbf{g}} P_{a}=$ $P_{b} \hat{\mathbf{g}} P_{b}=0$, chegamos a

$$
\left.\dot{\rho}(t)\right|^{f}=-i\left[P_{b} \hat{\mathbf{g}} P_{a} e^{-i \omega_{L} t}+P_{a} \hat{\mathbf{g}}^{\dagger} P_{b} e^{i \omega_{L} t}, \rho(t)\right]
$$

Esta expressão é a equação de Von Neumann para um hamiltoniano definido como $W=$ $P_{b} \hat{\mathbf{g}} P_{a} e^{-i \omega_{L} t}+P_{a} \hat{\mathbf{g}}^{\dagger} P_{b} e^{i \omega_{L} t}$. Podemos escrevê-lo de forma mais simples como

$$
\begin{aligned}
W & =\hbar V_{b a} e^{-i \omega_{L} t}+\hbar V_{a b} e^{i \omega_{L} t \omega_{L} t} \\
V_{b a} & =\Omega\left(\mathbf{e}_{L} \cdot \mathbf{Q}_{b a}\right) \\
\Omega & =\frac{1}{\hbar} E_{i n}\left|\left\langle E_{b} j_{b}\|\hat{d}\| E_{a} j_{a}\right\rangle\right|
\end{aligned}
$$

onde $V_{a b}=\left[V_{b a}\right]^{\dagger}$, $\Omega$ é a frequência de Rabi do sistema e $\mathbf{E}_{i n}=E_{i n} \mathbf{e}_{L}$. Como nos casos anteriores, a substituição do operador de campo livre se reflete, nos valores médios, como a interação com um campo clássico. 
A contribuição do primeiro termo de (5.5) é o hamiltoniano atômico livre,

$$
\begin{aligned}
\left.i \hbar \dot{\rho}(t)\right|^{A} & =\left[H_{0}, \rho(t)\right], \\
H_{0} & =\hbar \omega_{b} P_{b}+\hbar \omega_{a} P_{a},
\end{aligned}
$$

pois, como $\rho_{j i}=\left\langle\hat{\sigma}_{i j}\right\rangle$, a equação será a mesma a menos de um sinal que multiplica a frequência de Bohr. Isto fará com que a equação de Heisenberg para $\hat{\sigma}_{i j}$ seja uma equação de Von Neumann para $\rho_{i j}$.

\subsection{Equações de Bloch no Espaço de Liouville}

Nesta seção resolveremos as equações de Bloch no espaço de Liouville, que será discutido com mais detalhes no apêndice B. Estes métodos são usados regularmente em problemas com altas dimensões, uma introdução a estes métodos assim como aplicações podem ser encontradas em $[58,59]$. Reunindo todas as contribuições calculadas, a equação de Bloch fica

$$
\begin{aligned}
\frac{d \hat{\rho}}{d t} & \left.=\frac{1}{i \hbar}\left[H_{0}+H_{m}+W, \rho\right]+\frac{\partial \hat{\rho}}{\partial t}\right)_{d i s}, \\
\left.\frac{\partial \hat{\rho}}{\partial t}\right)_{d i s} & =-\frac{\Gamma}{2}\left\{P_{b}, \hat{\rho}(t)\right\}+\Gamma\left(2 j_{b}+1\right) \sum_{q=-1,0,1} \hat{Q}_{a b}^{\dagger q} \hat{\rho}(t) \hat{Q}_{b a}^{q}-\kappa\left(\rho-\rho_{0}\right) \\
H_{m} & =\left(\gamma_{a} P_{a}+\gamma_{b} P_{b}\right) \hat{J}_{z} \\
H_{0} & =\hbar \omega_{b} P_{b}+\hbar \omega_{a} P_{a} \\
W & =\hbar V_{b a} e^{-i \omega_{L} t}+\hbar V_{a b} e^{i \omega_{L} t}
\end{aligned}
$$

Motivados pela mudança de variáveis realizada nos capítulos anteriores, faremos a seguinte transformação,

$$
\begin{aligned}
& \hat{\rho}^{\prime}(t)=U(t) \hat{\rho}(t) U^{\dagger}(t), \\
& U(t)=P_{a}+P_{b} e^{i \omega_{L} t},
\end{aligned}
$$

que aberta em componentes fica

$$
\hat{\rho}^{\prime}(t)=P_{a} \hat{\rho}(t) P_{a}+P_{b} \hat{\rho}(t) P_{a}+P_{b} \hat{\rho}(t) P_{a} e^{i \omega_{L} t}+P_{a} \hat{\rho}(t) P_{b} e^{-i \omega_{L} t}
$$

Como cada $\hat{\sigma}_{m_{i} m_{j}}$ será uma base do espaço de Liouville, então o resultado é uma transformção semelhante a (3.37)-(3.38), mas para cada subespaço em vez de cada componente. 
No espaço de Liouville, $\hat{\rho}$ se torna

$$
\left.\left.\| \rho^{\prime}\right\rangle=\mathcal{L}(U) \mathcal{R}\left(U^{\dagger}\right) \| \rho\right\rangle
$$

com $R$ e $L$ sendo, respectivamente, os superoperadores referentes a operadores aplicados pela direita e pela esquerda. Devido à aditividade de $L$ e $R$,

$$
\mathcal{L}(U) \mathcal{R}\left(U^{\dagger}\right)=\mathcal{L}_{b} \mathcal{R}_{b}+\mathcal{L}_{a} \mathcal{R}_{a}+\mathcal{L}_{b} \mathcal{R}_{a} e^{i \omega_{L} t}+\mathcal{L}_{a} \mathcal{R}_{b} e^{-i \omega_{L} t}
$$

onde a $\mathcal{L}_{i}=\mathcal{L}\left(P_{i}\right)$, com $i=a, b$, e o mesmo vale para $\mathcal{R}_{i}$.

Note que a transformação (5.38) é diagonal no espaço de Liouville. Como $P_{i}=P_{i}^{n}$, com $n>1, \mathcal{L}_{i}=\mathcal{L}_{i}^{n}$ e $\mathcal{R}_{i}=\mathcal{R}_{i}^{n}$. Portanto,

$$
\mathcal{L}_{b} \mathcal{R}_{a} e^{i \omega_{L} t}+\mathcal{L}_{a} \mathcal{R}_{b} e^{-i \omega_{L} t}=\mathcal{L}_{b} \mathcal{R}_{a} e^{i \mathcal{L}_{b} \mathcal{R}_{a} \omega_{L} t}+\mathcal{L}_{a} \mathcal{R}_{b} e^{-i \mathcal{L}_{a} \mathcal{R}_{b} \omega_{L} t}
$$

então podemos escrevê-la na forma de uma exponencial,

$$
\begin{aligned}
\mathcal{L}(U) \mathcal{R}\left(U^{\dagger}\right) & =e^{i \mathcal{N} \omega_{L} t} \\
\mathcal{N} & =\mathcal{L}_{b} \mathcal{R}_{a}-\mathcal{L}_{a} \mathcal{R}_{b}=\mathcal{L}_{b}-\mathcal{R}_{b}
\end{aligned}
$$

onde a matriz $\mathcal{N}$ é diagonal, com elementos 0 nos subespaços $a a$ e $b b,-1$ em $a b$ e 1 em $b a$. Ela funciona como uma matriz de endereçamento. Note que definimos uma trasformação deste tipo de forma implicíta para os sistemas de dois e três níveis. Portanto,

$$
\left.\left.\left.i \hbar \frac{d}{d t} \| \rho^{\prime}(t)\right\rangle=e^{i \mathcal{N} \omega_{L} t} i \hbar \frac{d}{d t} \| \rho(t)\right\rangle-\hbar \mathcal{N} \omega_{L} \| \rho^{\prime}(t)\right\rangle
$$

Ao passar para o espaço de Liouville, a equação (5.32) fica,

$$
\begin{aligned}
\left.i \hbar \frac{d}{d t} \| \rho(t)\right\rangle= & \left\{\mathcal{L}\left(H_{0}+H_{m}+W\right)-\mathcal{R}\left(H_{0}+H_{m}+W\right)-i \hbar \frac{\Gamma}{2}\left[\mathcal{L}_{b}+\mathcal{R}_{b}\right]\right. \\
& \left.\left.+i \hbar \Gamma\left(2 j_{b}+1\right) \sum_{q=1,0,1} \mathcal{L}\left(\hat{Q}_{a b}^{\dagger q}\right) \mathcal{R}\left(\hat{Q}_{b a}^{q}\right)\right\} \| \rho(t)\right\rangle .
\end{aligned}
$$

Como $H_{0}=\hbar \omega_{b a} P_{b}-\hbar \omega_{a}$, então $\left[\exp \left(i \mathcal{N} \omega_{L} t\right), \mathcal{L}\left(H_{0}\right)-\mathcal{R}\left(H_{0}\right)\right]=0$. O hamiltoniano $H_{m}$ é muito parecido com $H_{0}$, tal que a diferença é o operador de momento angular. Este operador é diagonal na base $|i\rangle\langle j|$, portanto, a multiplicação por $\mathcal{L}_{a} \mathcal{R}_{b}$ ou $\mathcal{L}_{b} \mathcal{R}_{a}$ resultará em contribuição nula. Então $\mathcal{L}\left(J_{z}\right)$ e $\mathcal{R}\left(J_{z}\right)$ comutam com o superoperador (5.39). Para o hamiltoniano $W$

$$
\mathcal{L}(W)-\mathcal{R}(W)=\hbar \mathcal{L}\left(V_{a b}\right) e^{i \omega_{L} t}+\hbar \mathcal{L}\left(V_{b a}\right) e^{-i \omega_{L} t}-\hbar \mathcal{R}\left(V_{a b}\right) e^{i \omega_{L} t}-\hbar \mathcal{R}\left(V_{b a}\right) e^{-i \omega_{L} t}
$$


e podemos usar (5.38) para mostrar que

$$
e^{i \mathcal{N}\left(\omega_{L} t-\phi\right)}[\mathcal{L}(W)-\mathcal{R}(W)]=\hbar[\mathcal{L}(V)-\mathcal{R}(V)] e^{i \mathcal{N} \omega_{L}, t}
$$

onde $V=V_{a b}+V_{b a}$. Para as contribuições dissipativas radiativas, pode-se mostrar, aplicando $\mathcal{L}$ e $\mathcal{R}$ nos projetores, que estes termos também irão comutar com (5.39). Para $-\kappa\left(\rho-\rho_{0}\right)$, o primeiro termo será o próprio vetor no espaço de Liouville e o segundo termo será invariante pela transformação (5.39). Sendo assim, a equação de Bloch para as variáveis lentas no espaço de Liouville é

$$
\begin{aligned}
\frac{d \mathcal{X}(t)}{d t} & =\mathcal{M X}(t)+\kappa \mathcal{X}_{0} \\
\mathcal{M} & =i \mathcal{N} \Delta_{L}+\mathcal{H}_{m}+\mathcal{V}+\mathcal{R} \\
\mathcal{H}_{m} & =-\frac{i}{\hbar}\left[\mathcal{L}\left(\gamma_{a} P_{a} \hat{J}_{z}+\gamma_{b} P_{b} \hat{J}_{z}\right)+\mathcal{R}\left(\gamma_{a} P_{a} \hat{J}_{z}+\gamma_{b} P_{b} \hat{J}_{z}\right)\right] \\
\mathcal{R} & =-\frac{\Gamma}{2}\left[\mathcal{L}_{b}+\mathcal{R}_{b}\right]-\kappa \mathcal{I}+\Gamma\left(2 j_{b}+1\right) \sum_{q=1,0,1} \mathcal{L}\left(\hat{Q}_{a b}^{\dagger q}\right) \mathcal{R}\left(\hat{Q}_{b a}^{q}\right) \\
\mathcal{V} & =\Omega^{*} \mathcal{D}_{a b}+\Omega \mathcal{D}_{b a},
\end{aligned}
$$

onde $\mathcal{X}(t)$ é usado para designar $\left.\| \rho^{\prime}(t)\right\rangle, \Delta_{L}=\omega_{L}-\omega_{b a}$ é a dessintonia, $\mathcal{I}$ é o superoperador identidade e $D_{b a}$ e $D_{a b}$ são definidos como

$$
\begin{gathered}
\mathcal{D}_{a b}=-i\left[\mathcal{L}\left(\mathbf{e}_{L}^{*} \cdot \mathbf{Q}_{a b}\right)-\mathcal{R}\left(\mathbf{e}_{L}^{*} \cdot \mathbf{Q}_{a b}\right)\right] \\
\mathcal{D}_{b a}=-i\left[\mathcal{L}\left(\mathbf{e}_{L} \cdot \mathbf{Q}_{b a}\right)-\mathcal{R}\left(\mathbf{e}_{L} \cdot \mathbf{Q}_{b a}\right)\right]
\end{gathered}
$$

Esta equação é como (3.56) e pode ser solucionada da mesma maneira após retirar o vínculo introduzido pela normalização de $\rho(t)$. Desta forma, a solução estacionária é

$$
\overline{\mathcal{X}}=\mathcal{M}^{\prime-1}\left(\mathcal{B}+\mathcal{X}_{0}\right)
$$

onde $\mathcal{M}^{\prime}$ é uma matriz com uma dimensão a menos que $\mathcal{M}$ e livre de vínculos. $\mathcal{B}$ é um vetor que aparece no processo, como nos capítulos 3 e 4 .

A componente de frequência positiva do operador campo total na polarização $\mu$ que chega ao detetor será $\hat{\mathbf{E}}^{(+)}(t)=\left[\hat{E}_{\text {in }}^{(+)}(t)+i \beta \hat{P}_{q}^{(+)}(t)\right] \mathbf{e}_{\mu}$, onde $\hat{P}^{(+)}(t)$ é o operador polarização atômica definido em (3.61) e o campo incidente já está na polarização $\mu$. Neste caso, o mesmo será

$$
\hat{P}_{\mu}^{(+)}(t)=\sum_{m_{a} m_{b}}\left(\mathbf{e}_{\mu}^{*} \cdot \mathbf{d}_{m_{a} m_{b}}\right) \hat{\sigma}_{m_{a} m_{b}}(t)
$$

cuja média pode ser escrita como $\left\langle\hat{P}^{(+)}(t)\right\rangle=\operatorname{Tr}\left[\hat{d}_{a b}^{\mu} \rho(t)\right]$, onde $\hat{d}^{\mu}=\mathbf{e}_{\mu}{ }^{*} \cdot \hat{\mathbf{d}}$. A operação de traço é um produto escalar no espaço de Liouville, então a polarização média será dada 
por

$$
\left\langle\hat{P}_{\mu}^{(+)}(t)\right\rangle=\mathcal{U}^{T} \mathcal{L}\left(\hat{d}_{a b}^{\mu}\right) \mathcal{X}(t)
$$

onde ambos $\mathcal{U}$ e $\mathcal{U}^{T}$ são representações do operador identidade no espaço de Liouville com a mesma ordenação, porém um será uma matriz coluna e o outro será uma matriz linha. Note que $\mathcal{U}$ é diferente de $\mathcal{I}$, pois este é o superoperador identidade que atua no espaço de Liouville, enquanto aquele é um vetor defido neste mesmo espaço. A componente de frequência negativa pode ser encontrada tomando o complexo conjugado da expressão (5.48),

$$
\left\langle\hat{P}_{\mu}^{(-)}(t)\right\rangle=\mathcal{X}^{\dagger}(t)\left[\mathcal{L}\left(\hat{d}_{a b}^{\mu}\right)\right]^{\dagger} \mathcal{U}
$$

A mesma também pode ser encontrada por meio de $\hat{P}_{\mu}^{(-)}(t)=\sum_{m_{a} m_{b}}\left(\mathbf{e}_{\mu} \cdot \mathbf{d}_{m_{b} m_{a}}\right) \hat{\sigma}_{m_{b} m_{a}}(t)$, que fornecerá outras expressões para $\hat{P}_{\mu}^{( \pm)}(t)$,

$$
\begin{gathered}
\left\langle\hat{P}_{\mu}^{(-)}(t)\right\rangle=\mathcal{U}^{T} \mathcal{L}\left(\hat{d}_{b a}^{\mu}\right) \mathcal{X}(t), \\
\left\langle\hat{P}_{\mu}^{(+)}(t)\right\rangle=\mathcal{X}^{\dagger}(t)\left[\mathcal{L}\left(\hat{d}_{b a}^{\mu}\right)\right]^{\dagger} \mathcal{U}
\end{gathered}
$$

Podemos mostrar que o resultado final para $\hat{P}_{\mu}^{( \pm)}(t)$ é estritamente o mesmo independente obtido em (5.48) e (5.49) e esta mudança equivale a um rearranjo na soma. Usaremos uma ou outa forma conforme a conveniência.

Mostramos a seguir os gráficos 5.5 e 5.6 da absorção nas componentes de entrada e na componente perpendicular em função da varredura do campo magnético
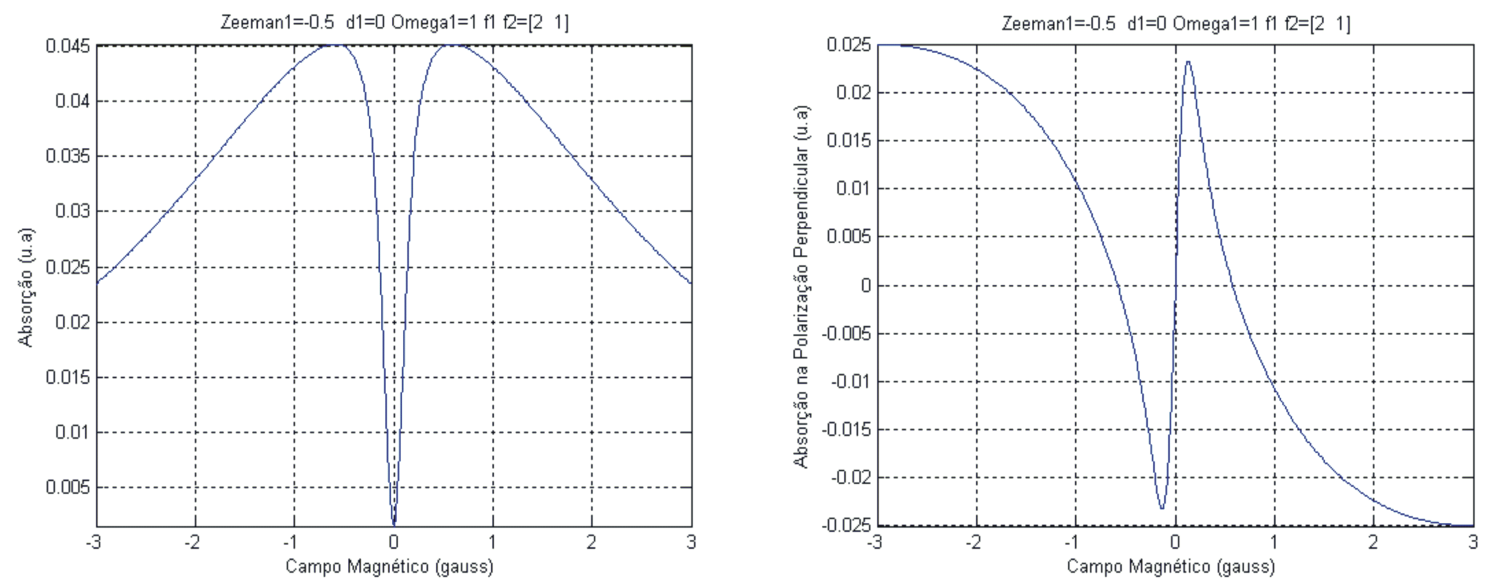

Figura 5.5: Absorção na componente de entrada e na perpendicular

Neles podemos ver que há realmente a assinatura no sinal de EIT no gráfico da absorção para a polarização do feixe laser e vemos um sinal de dispessão análogo ao mostrado no capítulo 4 para a outra componente de polarização. 

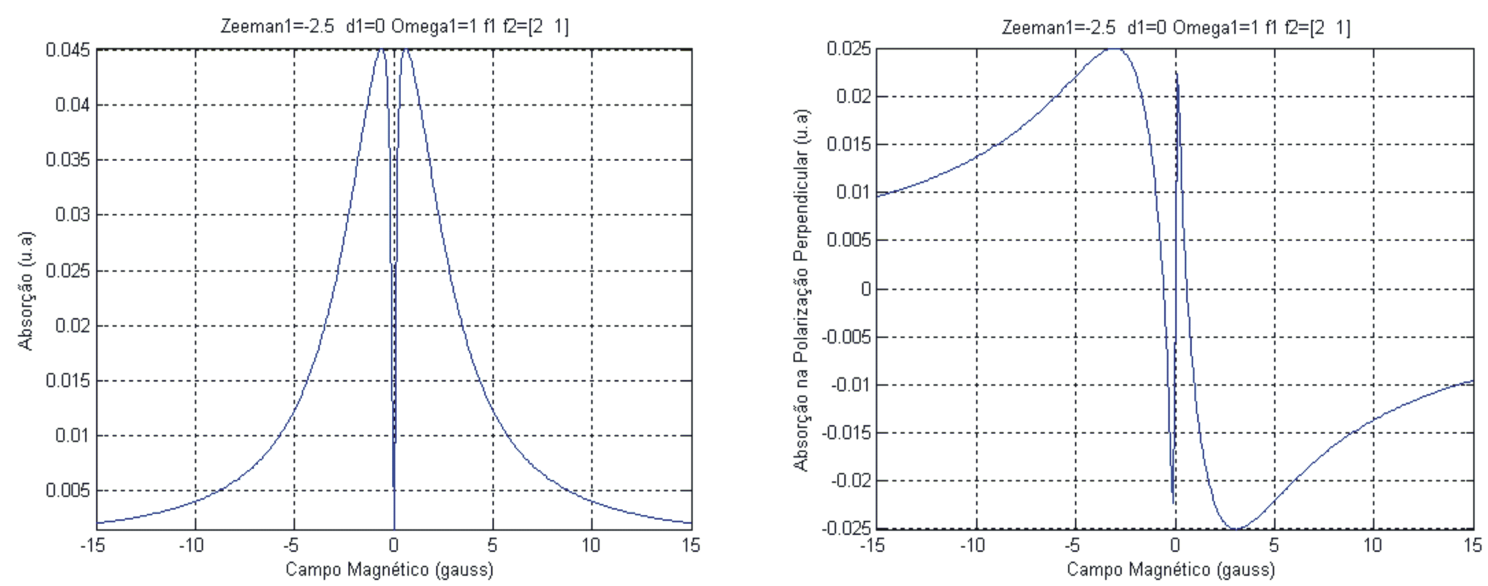

Figura 5.6: Absorção na componente de entrada e na perpendicular para uma excurção maior de campo magnético

\subsection{Equação para as Flutuações e Espectro de Ruído}

Faremos novamente um modelo linearizado para as flutuações,

$$
\begin{aligned}
\hat{\sigma}_{i j}(t) & =\bar{\rho}_{j i}+\delta \hat{\sigma}_{i j}(t), \\
\hat{\mathbf{E}}_{i n}^{(+)}(t) & =\mathbf{E}_{i n}+\delta \hat{\mathbf{E}}_{i n}^{(+)}(t) .
\end{aligned}
$$

Vamos mostrar qual equação as flutuações satisfazem. De todas as contribuições calculadas, apenas uma contém explicitamente o campo incidente e iniciaremos por ela. Escrevemos a equação (5.25) para $\delta \hat{\sigma}_{i j}(t)$ sem levar em conta as forças de Langevin

$$
\begin{aligned}
\left.\delta \dot{\hat{\sigma}}_{i j}(t)\right|^{f}= & \frac{1}{i \hbar} \sum_{m_{a}}\left[\hat{\sigma}_{i m_{a}}(t) \mathbf{d}_{j m_{a}} \cdot \mathbf{E}_{i n} e^{-i \omega_{L} t}-\mathbf{d}_{m_{a} i} \cdot \mathbf{E}_{i n}^{*} e^{i \omega_{L} t} \hat{\sigma}_{m_{a} j}(t)\right] \\
& -\frac{1}{i \hbar} \sum_{m_{b}}\left[\hat{\sigma}_{j m_{b}}(t) \mathbf{d}_{m_{b} i} \cdot \mathbf{E}_{i n} e^{-i \omega_{L} t}-\mathbf{d}_{m_{b} j} \cdot \mathbf{E}_{i n}^{*} e^{i \omega_{L} t} \hat{\sigma}_{i m_{b}}(t)\right] .
\end{aligned}
$$

Esta equação possui os mesmos coeficientes que (5.27). Portanto, definimos a ordenação do vetor ao passar para o espaço de Liouville tal que cada elemento $\hat{\sigma}_{i j}(t)$ corresponda a $\rho_{j i}$. No espaço de Liouville, a equação (5.54), após passar para as variáveis lentas, será

$$
\left.\delta \dot{\mathbf{X}}(t)\right|^{f 1}=\mathcal{V} \delta \mathbf{X}(t)
$$

A contribuição da flutuação inserida pelo campo incidente em (5.25) será

$$
\begin{aligned}
\left.\delta \dot{\hat{\sigma}}_{i j}(t)\right|^{f}= & \frac{1}{i \hbar} \sum_{m_{a}}\left[\rho_{m_{a} i}(t) \mathbf{d}_{j m_{a}} \cdot \delta \hat{\mathbf{E}}_{i n}^{(+)} e^{-i \omega_{L} t}-\mathbf{d}_{m_{a} i} \cdot \delta \hat{\mathbf{E}}_{i n}^{(-)} e^{i \omega_{L} t} \rho_{j m_{a}}(t)\right] \\
& -\frac{1}{i \hbar} \sum_{m_{b}}\left[\rho_{j m_{b}}(t) \mathbf{d}_{m_{b} i} \cdot \delta \hat{\mathbf{E}}_{i n}^{(+)} e^{-i \omega_{L} t}-\mathbf{d}_{j m_{b}} \cdot \delta \hat{\mathbf{E}}_{i n}^{(-)} e^{i \omega_{L} t} \rho_{m_{b} i}(t)\right]
\end{aligned}
$$


Esta equação possui coeficientes parecidos com os de (5.27), porém com $\delta \hat{\mathbf{E}}_{i n}^{(+)}$no lugar de $\mathbf{E}_{i n}$. No espaço de Liouville, esta equação será

$$
\begin{aligned}
\left.\delta \dot{\mathbf{X}}(t)\right|^{f 2} & =\eta\left[\delta \hat{\mathbf{E}}_{i n}^{(-)} \mathcal{D}_{a b}+\delta \hat{\mathbf{E}}_{i n}^{(+)} \mathcal{D}_{b a}\right] \\
\eta & =\frac{\left|\left\langle E_{b} j_{b}\|\hat{d}\| E_{a} j_{a}\right\rangle\right|}{\hbar}
\end{aligned}
$$

As contribuições dissipativas e do átomo livre dependem apenas de $\delta \hat{\sigma}_{i j}(t)$. Ao passar para o espaço de Liouville, as equações para ambas manterão a mesma forma que aquela satisfeita pelos os valores médios. A interação com o campo magnético já foi introduzida para os valores médios, mas a equação de Heisenberg para $\hat{\sigma}_{i j}(t)$ deve possuir os mesmos coeficientes que a equação de Von Neumann para $\rho_{j i}(t)$, portanto a contribuição para $\delta \hat{\sigma}_{i j}(t)$ também deve satisfazer a mesma equação que $\rho_{j i}(t)$. As forças de Langevin, como possuem média nula, apenas irão contribuir para a equação da flutuação. O termo de decaimento não radiativo $\kappa \rho_{0}$ não flutua e contribuirá apenas para o valor médio. Sendo assim, a equação para as flutuações fica

$$
\frac{d}{d t} \delta \hat{\mathcal{X}}(t)=\mathcal{M} \delta \hat{\mathcal{X}}(t)+\eta\left[\delta \hat{\mathbf{E}}_{i n}^{(-)}(t) \mathcal{D}_{a b}+\delta \hat{\mathbf{E}}_{i n}^{(+)}(t) \mathcal{D}_{b a}\right] \overline{\mathcal{X}}+\hat{\mathcal{F}}(t),
$$

que, junto com (5.40), determina a dinâmica do sistema até a segunda ordem nas flutuações. No domínio da frequência, a solução para $\delta \hat{\mathcal{X}}(\omega)$ é

$$
\delta \hat{\mathcal{X}}(\omega)=[i \omega-\mathcal{M}]^{-1}\left[\eta\left(\delta \hat{\mathbf{E}}_{i n}^{(-)}(\omega) \mathcal{D}_{a b}+\delta \hat{\mathbf{E}}_{i n}^{(+)}(\omega) \mathcal{D}_{b a}\right) \overline{\mathcal{X}}+\hat{\mathcal{F}}(\omega)\right]
$$

Podemos escrever a flutuação do campo na polarização $\mu$ como $\delta \hat{E}_{\text {in }}^{(+)}(t)=\hat{p}(t)+$ $i \hat{\mu}(t)$, onde $\hat{p}(t)$ e $\hat{q}(t)$ são hermitianos. Para o caso em que $E_{\text {in }}$ é real $\hat{p}(t)$ e $\hat{q}(t)$ serão, respectivamente, a flutuação de amplitude e fase. A partir da expressão (5.48), a flutuação na polarização $\mu$ fica

$$
\begin{aligned}
& \delta \hat{P}_{\mu}^{(-)}(t)=\delta \hat{\mathcal{X}}^{\dagger}(t)\left[\mathcal{L}\left(\hat{d}_{a b}^{\mu}\right)\right]^{\dagger} \mathcal{U}=\mathcal{U}^{T} \mathcal{L}\left(\hat{d}_{b a}^{\mu}\right) \delta \hat{\mathcal{X}}(t) \\
& \delta \hat{P}_{\mu}^{(+)}(t)=\delta \hat{\mathcal{X}}^{\dagger}(t)\left[\mathcal{L}\left(\hat{d}_{b a}^{\mu}\right)\right]^{\dagger} \mathcal{U}=\mathcal{U}^{T} \mathcal{L}\left(\hat{d}_{a b}^{\mu}\right) \delta \hat{\mathcal{X}}(t)
\end{aligned}
$$

Note que nas equações (5.48)-(5.51) operação de conjugação hermitiana é, como de constume, uma transposição seguida de uma conjugação complexa, pois cada elemento de $\mathcal{X}(t)$ é um número complexo. Mas como os elementos de $\delta \hat{\mathcal{X}}(t)$ são operadores, podemos mostrar que tomar o seu hermitiano conjugado equivale a realizar a transposição do vetor e tomar o hermitiano conjugado de cada elemento. 
No domínio da frequência, as flutuações nas componentes de frequência positiva e negativa do campo incidente e da polarização serão, respectivamente,

$$
\begin{aligned}
\delta \hat{E}_{i n}^{(+)}(\omega) & =\hat{p}(\omega)+i \hat{q}(\omega), \\
\delta \hat{E}_{i n}^{(-)}(\omega) & =\hat{p}(\omega)-i \hat{q}(\omega), \\
\delta \hat{P}_{\mu}^{(+)}(\omega) & =\delta \hat{\mathcal{X}}^{\dagger}(\omega)\left[\mathcal{L}\left(\hat{d}_{b a}^{\mu}\right)\right]^{\dagger} \mathcal{U}=\mathcal{U}^{T} \mathcal{L}\left(\hat{d}_{a b}^{\mu}\right) \delta \hat{\mathcal{X}}(\omega), \\
\delta \hat{P}_{\mu}^{(-)}(\omega) & =\delta \hat{\mathcal{X}}^{\dagger}(\omega)\left[\mathcal{L}\left(\hat{d}_{a b}^{\mu}\right)\right]^{\dagger} \mathcal{U}=\mathcal{U}^{T} \mathcal{L}\left(\hat{d}_{b a}^{\mu}\right) \delta \hat{\mathcal{X}}(\omega) .
\end{aligned}
$$

Substituindo (5.62) e (5.63) em (5.58), podemos escrevê-la em termos de $\hat{p}(\omega)$ e $\hat{q}(\omega)$,

$$
\begin{aligned}
\delta \hat{\mathcal{X}}(\omega) & =\mathcal{G}_{p}(\omega) \hat{p}(\omega)+\mathcal{G}_{q}(\omega) \hat{q}(\omega)+\mathcal{G}_{f}(\omega) \hat{\mathcal{F}}(\omega) \\
\mathcal{G}_{p}(\omega) & =\eta[i \omega-\mathcal{M}]^{-1}\left[\mathcal{D}_{b a}+\mathcal{D}_{a b}\right] \overline{\mathcal{X}} \\
\mathcal{G}_{q}(\omega) & =i \eta[i \omega-\mathcal{M}]^{-1}\left[\mathcal{D}_{b a}-\mathcal{D}_{a b}\right] \overline{\mathcal{X}} \\
\mathcal{G}_{f}(\omega) & =[i \omega-\mathcal{M}]^{-1}
\end{aligned}
$$

Portanto, a expressão para a flutuação da componente de frequência positiva do campo total na polarização $\mu$ se torna, utilizando uma das expressões para a polarização,

$$
\begin{aligned}
\delta \hat{E}_{\mu}^{(+)}(\omega) & =\hat{p}(\omega) \mathcal{K}_{p}^{\mu}(\omega)+\hat{q}(\omega) \mathcal{K}_{q}^{\mu}(\omega)+\hat{\mathcal{F}}^{\dagger}(\omega) \mathcal{K}_{f}^{\mu}(\omega) \\
\mathcal{K}_{p}^{\mu}(\omega) & =1+\mathcal{G}_{p}^{\dagger}(\omega)\left[\mathcal{L}\left(\hat{d}_{b a}^{\mu}\right)\right]^{\dagger} \mathcal{U} \\
\mathcal{K}_{q}^{\mu}(\omega) & =i+\mathcal{G}_{q}^{\dagger}(\omega)\left[\mathcal{L}\left(\hat{d}_{b a}^{\mu}\right)\right]^{\dagger} \mathcal{U} \\
\mathcal{K}_{f}^{\mu}(\omega) & =\mathcal{G}_{f}^{\dagger}(\omega)\left[\mathcal{L}\left(\hat{d}_{b a}^{\mu}\right)\right]^{\dagger} \mathcal{U}
\end{aligned}
$$

ou, de forma equivalente e utilizando a outra expressão para a polarização, pode ser escrita como

$$
\begin{aligned}
\delta \hat{E}_{\mu}^{(+)}(\omega) & =\mathcal{K}_{p}^{\prime \mu}(\omega) \hat{p}(\omega)+\mathcal{K}_{q}^{\prime \mu}(\omega) \hat{q}(\omega)+\mathcal{K}_{f}^{\prime \mu}(\omega) \hat{\mathcal{F}}(\omega) \\
\mathcal{K}_{p}^{\prime \mu}(\omega) & =1+\mathcal{U}^{T} \mathcal{L}\left(\hat{d}_{a b}^{\mu}\right) \mathcal{G}_{p}(\omega) \\
\mathcal{K}_{q}^{\prime \mu}(\omega) & =i+\mathcal{U}^{T} \mathcal{L}\left(\hat{d}_{a b}^{\mu}\right) \mathcal{G}_{q}(\omega) \\
\mathcal{K}_{f}^{\prime \mu}(\omega) & =\mathcal{U}^{T} \mathcal{L}\left(\hat{d}_{a b}^{\mu}\right) \mathcal{G}_{f}(\omega)
\end{aligned}
$$

e flutuação para a componente de frequência negativa será $\delta \hat{E}_{\mu}^{(-)}(\omega)=\left[\delta \hat{E}_{\mu}^{(+)}(-\omega)\right]^{\dagger}$.

Como $\left\langle\hat{p}(\omega) \mathcal{F}_{i}(\omega)\right\rangle=\left\langle\hat{q}(\omega) \mathcal{F}_{i}(\omega)\right\rangle=0$, as funções de correlação definidas em (3.76) 
serão

$$
\begin{aligned}
\left\langle\delta \hat{E}_{\mu^{\prime}}^{(+)}(\omega) \delta \hat{E}_{\mu}^{(+)}(-\omega)\right\rangle & =\mathcal{K}_{p}^{\prime \mu^{\prime}}(\omega) \mathcal{K}_{p}^{\mu}(-\omega)\langle\hat{p}(\omega) \hat{p}(-\omega)\rangle+\mathcal{K}_{q}^{\prime \mu^{\prime}}(\omega) \mathcal{K}_{q}^{\mu}(-\omega)\langle\hat{q}(\omega) \hat{q}(-\omega)\rangle \\
& +\mathcal{K}_{q}^{\prime \mu^{\prime}}(\omega) \mathcal{K}_{p}^{\mu}(-\omega)\langle\hat{q}(\omega) \hat{p}(-\omega)\rangle+\mathcal{K}_{p}^{\prime \mu^{\prime}}(\omega) \mathcal{K}_{q}^{\mu}(-\omega)\langle\hat{p}(\omega) \hat{q}(-\omega)\rangle \\
& +\mathcal{K}_{f}^{\prime \mu^{\prime}}(\omega)\left\langle\mathcal{F}(\omega) \mathcal{F}^{\dagger}(-\omega)\right\rangle \mathcal{K}_{f}^{\mu}, \\
\left\langle\delta \hat{E}_{\mu^{\prime}}^{(-)}(\omega) \delta \hat{E}_{\mu}^{(+)}(-\omega)\right\rangle= & {\left[\mathcal{K}_{p}^{\mu^{\prime}}(-\omega)\right]^{\dagger} \mathcal{K}_{p}^{\prime \mu}(-\omega)\langle\hat{p}(\omega) \hat{p}(-\omega)\rangle+\left[\mathcal{K}_{q}^{\mu^{\prime}}(-\omega)\right]^{\dagger} \mathcal{K}_{q}^{\prime \mu}(-\omega)\langle\hat{q}(\omega) \hat{q}(-\omega)\rangle } \\
+ & {\left[\mathcal{K}_{q}^{\mu^{\prime}}(-\omega)\right]^{\dagger} \mathcal{K}_{p}^{\prime \mu}(-\omega)\langle\hat{q}(\omega) \hat{p}(-\omega)\rangle+\left[\mathcal{K}_{p}^{\mu^{\prime}}(-\omega)\right]^{\dagger} \mathcal{K}_{q}^{\prime \mu}(-\omega)\langle\hat{p}(\omega) \hat{q}(-\omega)\rangle } \\
& +\left[\mathcal{K}_{f}^{\mu^{\prime}}(-\omega)\right]^{\dagger}\left\langle\mathcal{F}(\omega) \mathcal{F}^{\dagger}(-\omega)\right\rangle \mathcal{K}_{f}^{\prime \mu}(-\omega) .
\end{aligned}
$$

Sendo assim, o problema de encontrar o espectro de ruído se resume a calcular as correlações $\langle\hat{p}(\omega) \hat{p}(-\omega)\rangle,\langle\hat{q}(\omega) \hat{q}(-\omega)\rangle,\langle\hat{q}(\omega) \hat{p}(-\omega)\rangle$ e a matriz de difusão $\langle\mathcal{F}(\omega) \mathcal{F}(-\omega)\rangle$.

Como fazemos nossa espectroscopia com lasers de diodo, o ruído de fase inserido por este laser é muito maior que o ruído de intensidade e também muito maior que o introduzido pelos modos do vácuo na forma das forças de Langevin. Neste aproximação podemos considerar $\left\langle\mathcal{F}_{i}(\omega) \mathcal{F}_{j}(-\omega)\right\rangle=0$, onde $i$ e $j$ são as componentes no espaço de Liouville e $\langle\hat{p}(\omega) \hat{p}(-\omega)\rangle=0$. Também consideraremos que fase e amplitude são descorrelacionadas, $\langle\hat{p}(\omega) \hat{q}(-\omega)\rangle=0$, o que não é estritamente verdadeiro para um laser de diodo, mas este termo irá contribuir muito menos que a função de covariância de fase. Portanto, apenas $\langle\hat{q}(\omega) \hat{q}(-\omega)\rangle$ será considerada na análise. Nesta aproximação, o espectro de ruído se torna

$$
S_{\mu, \mu^{\prime}}(\omega)=\left[\left[\mathcal{K}_{q}^{\prime \mu^{\prime}}(-\omega)\right]^{\dagger} \mathcal{K}_{q}^{\mu}(-\omega)+\mathcal{K}_{q}^{\prime \mu^{\prime}}(\omega) \mathcal{K}_{q}^{\mu}(-\omega)+\text { c.c. }\right]\langle\hat{q}(\omega) \hat{q}(-\omega)\rangle .
$$

As matrizes $\mathcal{K}_{q}^{\prime \mu}(\omega)$ e $\mathcal{K}_{q}^{\mu}(\omega)$ podem ser calculadas diretamente por métodos numéricos, pois são funções somente do estado estacionário e de representações de operadores no espaço de Liouville. As medidas e os resultados para a deteção homodina serão apresentados no capítulo sobre os resultados obtidos. No próximo capítulo, faremos uma descrição detalhada sobre os métodos experimentais utilizados diretamente para a obtenção dos dados e para controle do experimento. 


\section{$6 \quad$ Métodos Experimentais}

Este capítulo é voltado para os métodos usados para controle e realização das medidas no laboratório. Daremos uma breve explicação de como funciona o laser de diodo que usamos. Trataremos do sistema de controle, que utiliza uma célular de vapor auxiliar para comparar a frequência do laser diretamente com as frequências de transição atômica, por meio da técnica de absorção saturada. Descreveremos como ocorre o travamento da frequência do laser nos picos de absorção, por meio do sistema de Lock-in, assim como os moduladores acusto-óticos, que geram deslocamentos na frequência do laser. O método da detecção homodina, utilizado para calibrar o shot noise ${ }^{1}$ e realizar as medidas de soma e subtração dos feixes, também será tratado aqui. Faremos também uma breve descrição do analisador de espectro, que é o aparelho utilizado para medir as flutuações no domínio da frequência. Uma descrição mais detalhada sobre boa parte do que é tratado neste capítulo pode ser encontrada em [60, 25]. Iniciaremos com algumas considerações sobre o elemento químico usado no experimento, o rubídio, e seus níveis hiperfinos.

\section{1 Átomo de Rubídio}

O Rubídio é um átomo hidrogenóide pertencente à família 1A da tabela periódica, de número atômico 37 e massa atômica 85,5u. Ele foi descoberto por Bunsen e Kirchhoff em 1861 e seu nome vem do latim "rubidus", que significa vermelho escuro. Existem dois isótopos de Rubídio na natureza: o ${ }^{85} R b$, mais abundante e que corresponde a $72,2 \%$ da quantidade total o elemento e o ${ }^{87} R b$, responsável pelos outros $27,8 \%$. Os spins do nucleares para os isótopos são $I_{87}=\frac{3}{2}$ e $I_{85}=\frac{5}{2}$.

No nosso experimento, trabalhamos na linha $D_{2}$ do $R b^{85}$, cujo comprimento de onda é $\lambda=780,241 \mathrm{~nm}$ e está esquematizada na figura 6.1. O átomo de Rubídio é bastante usado em experimentos de Ótica Quântica e Física Atômica por causa da sua estrutura

\footnotetext{
${ }^{1}$ É o ruído quântico padrão, que ocorre pela presença da luz e é gerado pelo caráter corpuscular da mesma.
} 
eletrônica. Como todos os elementos da família 1A ele possui apenas um elétron na camada de valência. Isto faz com que a sua estrutura eletrônica seja muito parecida com a do hidrogênio, validando muitas das teorias utilizadas, inclusive a descrita neste trabalho. Outro grande motivo para se utilizar o Rubídio é que ambas as linhas $D_{2}$ e $D_{1}$ são acessíveis por meio de lasers de diodo, que são os mais comuns nos laboratórios de Física Atômica, e foram utilizados como campo incidente neste trabalho.

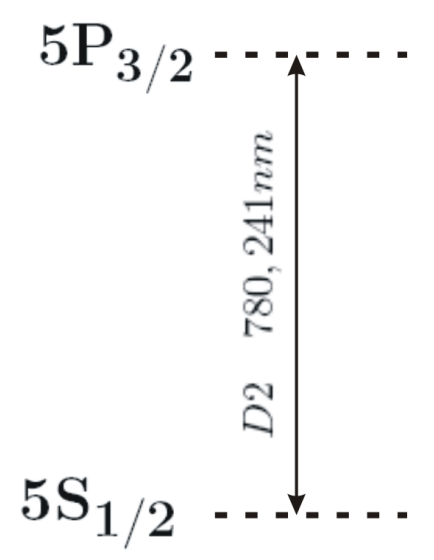

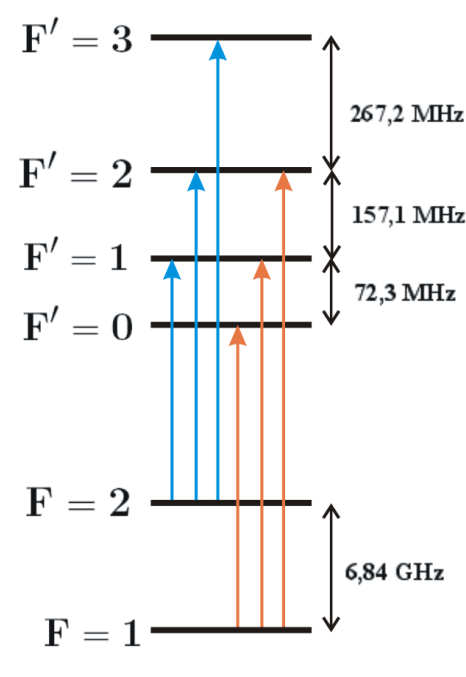

$\mathrm{Rb}^{87}$

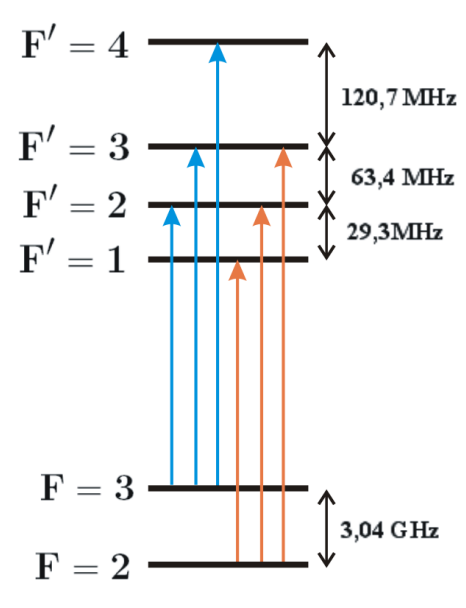

$\mathrm{Rb}^{85}$

Figura 6.1: Estrutura Hiperfina da Linha $D_{2}$ do Ruibídio

Na figura (6.1) podemos ver as transições permitidas, que se referem a $\Delta J=0, \pm 1$. Por serem desdobramentos do estado de estrutura fina $5 S_{1 / 2}$, os estados $F=1$ e $F=2$ do $R b^{87}$ e $F=2$ e $F=3$ do ${ }^{85} R b$ correspondem ao menor momento angular orbital, $l=0$. Usamos aqui a denominação estado fundamental para designar os estados estáveis, por isso, ambos serão chamados de estados fundamentais, apesar de um deles possuir energia menor que o outro. Nas medidas utilizando dois feixes, cuja teoria é tratada no capítulo 4 os níveis $|1\rangle$ e $|3\rangle$ correspondem a estes níveis fundamentais.

\subsection{Alargamento Doppler}

Os resultados mostrados no capítulo (3) para absorção são derivados supondo que o átomo está parado. Situação que não ocorre numa célula de vapor, onde há átomos com diversas velocidades, que estão distribuídas conforme a distribuição de Maxwell- 
Boltzmann,

$$
p(\mathbf{v}) d \mathbf{v}=\frac{1}{\sqrt{\pi^{3}} V v_{p}^{3}} e^{-\frac{\mathbf{v}^{2}}{v_{p}^{2}}} d \mathbf{v},
$$

onde $v_{p}=\sqrt{2 k_{b} T / m}=\sqrt{3 / 2} v_{m}$ é a velocidade mais provável, com $v_{m}^{2}$ sendo a velocidade quadrática média, $k_{b}$ a constante de Boltzman, $m$ a massa de cada átomo, $T$ a temperatura e $V$ o volume considerado. Sua inclusão é necessária para a manter a dimensão correta e aparece ao calcular a função de partição do sistema. A velocidade dos átomos faz com que estes percebam o campo com um deslocamento na sua frequência provocado pelo efeito Doppler,

$$
\omega_{L}^{\prime}=\omega_{L}-\mathbf{k} \cdot \mathbf{v}
$$

Apesar deste deslocamento ser pequeno, pois depende do inverso da velocidade da luz, o mesmo pode ser suficientemente grande em comparação á separação dos níveis hiperfinos, que são correções de ordem $10^{3}$ inferiores às correções relativísticas para o átomo de hidrogênio. Por exemplo, um átomo se movimentando com a velocidade mais provável pela distribuição de Maxwell à temperatura ambiente, perceberia o laser com um deslocamento em frequência de $\Delta f 309 M h z^{2}$. A curva de absorção é dada por (??) e como $\omega_{L}^{\prime}$ depende da velocidade, devemos calcular a absorção média usando (6.1) como densidade de probabilidade,

$$
A b s=\frac{\Omega \Gamma N}{\sqrt{\pi^{3} V} v_{p}^{3}} \int d^{3} v \frac{e^{-\frac{\mathbf{v}^{2}}{v_{p}^{2}}}}{2 \Omega^{2}+(\delta-\mathbf{k} \cdot \mathbf{v})^{2}+\left(\frac{\Gamma}{2}\right)^{2}},
$$

onde $N$ é o número total de átomos que interagem com o laser. Como apenas a componente da velocidade dos átomos na direção do feixe contribui para o sinal de absorção, escolhemos a direção de propagação do feixe como sendo o eixo $z$ do sistema de coordenadas, portanto,

$$
A b s=\frac{\Omega \Gamma n}{\sqrt{\pi} v_{p}} \int_{-\infty}^{\infty} d v \frac{e^{-\frac{v^{2}}{v_{p}^{2}}}}{2 \Omega^{2}+\left(\delta-\frac{\omega_{L}}{c} v\right)^{2}+\left(\frac{\Gamma}{2}\right)^{2}}
$$

onde $n=N / V$ é a densidade de átomos. Vamos transformar a integral em velocidade acima em uma integral em frequência $\operatorname{com} \omega=\omega_{L} v / c$,

$$
A b s=\frac{\Omega \Gamma n c}{\sqrt{\pi} v_{p} \omega_{L}} \int_{-\infty}^{\infty} d \omega \frac{e^{-\left(\frac{c}{\omega_{L} v_{p}}\right)^{2} \omega^{2}}}{(\omega-\delta)^{2}+2 \Omega^{2}+\left(\frac{\Gamma}{2}\right)^{2}} .
$$

Este sinal de absorção é a soma de lorentzianas centradas em dessintonia nula com peso dado por uma gaussiana centrada na origem. A largura das lorentizanas é dada em

\footnotetext{
${ }^{2}$ Conseguimos ter controle na frequência dos lasers da ordem de $10 \mathrm{MHz}$.
} 
(3.55) e a largura do envoltorio gaussiano será

$$
\Delta \omega_{G}=\frac{\omega_{L} v_{p}}{2 c}
$$

Em nosso caso, esta largura é $\Delta f=309 \mathrm{MHz}$, que é muito maior que a largura natural. Desta forma, as lorentzianas podem ser aproximadas por deltas de Dirac e o perfil da curva de absorção será gaussiano para dessintonia próxima de zero. Para grandes dessintonias, pelo fato das lorentzianas cairem lentamente para $|\omega|$ grande, a curva terá perfil lotenziano.

A largura da curva obtida pode ser calculada numéricamente e será da ordem de $\Delta f_{\text {Doppler }}=517 M h z$, ou seja, $\Delta f_{\text {Doppler }} \approx 100 \Gamma$. Como pode ser visto na figura 6.1 , as diferenças entre os níveis excitados na linha $D_{2}$ de ambos ${ }^{87} R b$ e ${ }^{85} R b$ são menores que a largura Doppler, por isso não podemos resolver as transições hiperfinas com apenas um laser incidindo sobre os átomos. Desta forma, se faz necessário usar de métodos alternativos para eliminar o alargamento Doppler na espectroscopia. O método utilizado neste trabalho é a absorção saturada, que é tema da próxima seção.

\subsection{Absorção Saturada}

Como mostrado na seção anterior, o efeito Doppler gera um alargamento no sinal de absorção para medidas feitas em uma célula de vapor. Para superar este problema, usamos a técnica da absorção saturada, que consiste em incidir dois feixes de mesma frequência, direção de propagação e sentidos opostos sobre a célula ${ }^{3}$. Considere um átomo interagindo com dois campos, com vetor de onda $\mathbf{k}_{i}, i=1,2$, como mostrado na figura 6.2a). A dessintonia para cada átomo será $\delta_{i}=\delta-\mathbf{k}_{i} \cdot \mathbf{v}$, então a diferença entre as dessintonias será

$$
\delta_{2}-\delta_{1}=\left(\mathbf{k}_{1}-\mathbf{k}_{2}\right) \cdot \mathbf{v}
$$

Caso apenas um feixe incidisse nos átomos observaríamos o sinal de absorção alargado pelo efeito Doppler. Com dois feixes ocorre o mesmo fenômeno, porém, à medida que varremos os fexes e estes passam por dessintonia nula, ocorre a saturação da transição. O coeficiente de absorção depende da intensidade do feixe, proporcional ao quadrado da frequência de Rabi, como em (3.52). A absorção cresce linearmente com a frequência de Rabi para baixas intensidades, chega a um máximo e cai lentamente para $\Omega \longrightarrow \infty$. Esta

\footnotetext{
${ }^{3} \mathrm{Na}$ prática costuma-se usar o mesmo feixe tal que este seja reincidido sobre si mesmo no sentido oposto.
} 
a)

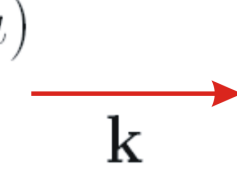

b)

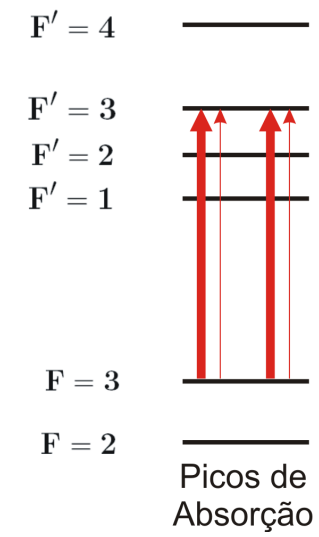

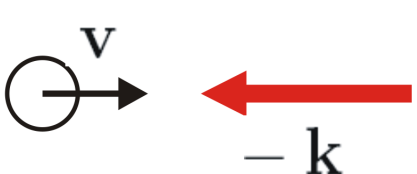

$-\mathrm{k}$

Figura 6.2: a) Átomo com velocidade $\mathbf{v}$ interagindo com dois feixes contrapropagantes. b) Diagrama de níveis do rubídio mostrado a interação com os feixes intenso e fraco, assim como as situações em que ocorrem os picos de absorção e crossover

queda ocorre devido ao fenômeno da saturação, em que a população está ingualmente distribuida entre o estado fundamental e o excitado. Mostramos um gráfico da absorção em função de $\Omega$ para $\delta=0$ e $\delta=3 \Gamma$. A intensidade de saturação é definida como aquela em que o coeficiente de absorção é metade do seu valor máximo. Esta intensidade será menor quanto mais o feixe se aproxima da ressonância com pode ser visto no gráfico 6.3. Nesta intensidade, praticamente metade da população estará no nível excitado.

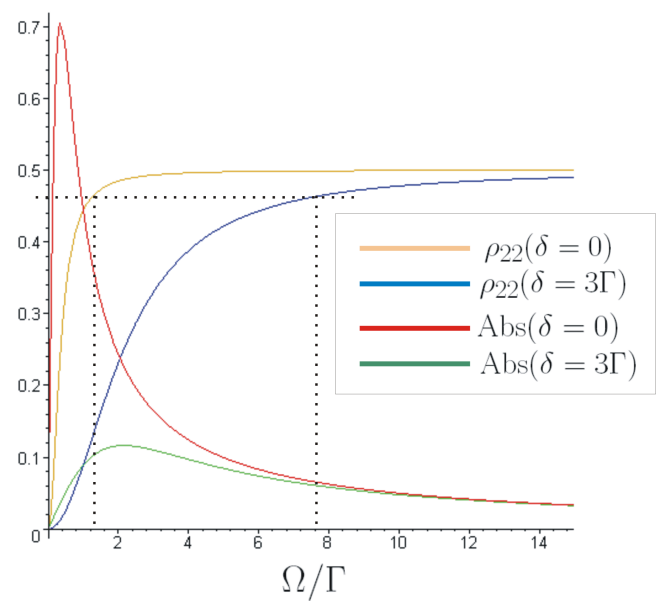

Figura 6.3: Curvas de absorção e população do estado excitado em função da frequência de Rabi para dessintonia nula e para $\delta=3 \Gamma$

Apenas átomos com velocidades nulas na direção $z$ percebem ambos os feixes com a mesma frequência. Se a soma das intensidades dos dois feixes for maior que a intensidade 
de saturação, quando o laser passar pela ressonância haverá uma queda na absorção. Estas quedas serão pequenas depleções no sinal de absorção, com largura bem menor que a do perfil Doppler. Este sinal separa os níveis hiperfinos que estão na mesma largura Doppler, como é o caso da linha $D_{2}$ do rubídio. Como apenas átomos com velocidade nula na direção do feixe contribuem para o sinal de absorção saturada numa dada transição, a queda na absorção será pequena em comparação com a absorção total. Uma varredura na linha $D_{2}$ com o esquema de absorção saturada é mostrada na Figura 6.4.

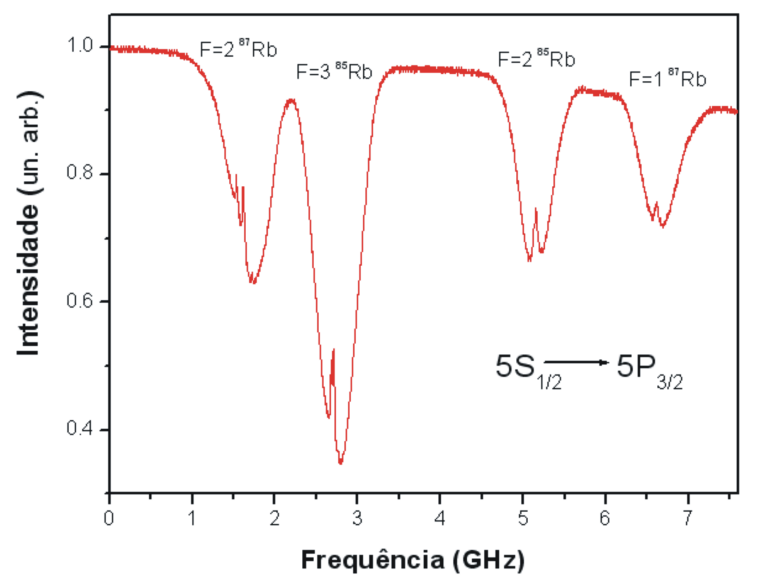

Figura 6.4: Espectroscopia de absorção saturada da linha D2 do rubidio. Os picos representa as duas transições dos isótopos presentes na célula. Conseguimos ver as pequenas quedas de absorção saturadas em cada pico.

Pode ocorrer também que o átomo esteja com uma velocidade tal que perceba os feixe ressonantes com transições diferentes que tenham o nível fundamental em comum. Desta forma, podemos considerar o átomo em um sistema de três níveis em $V$ sujeito à condição de ressonância para os dois feixes. Neste caso, a população no estado fundamental irá diminuir, por causa da ressonância de ambos os feixes, o que levará a uma redução na absorção. As depleções que ocorrem nesta frequência são chamadas picos de cros-over e ocorrem quando a frequência do laser é a média das frequências de Bohr das duas transições. Os picos de cross-over são geralmente maiores que os referentes às transições, pois existem mais átomos com esta velocidade na direção $z$ do que com velocidade nula.

O sinal de absorção saturada é usado para monitorar e controlar a frequência dos lasers. Quando a frequência é varrida pouco em torno de uma transição, o sinal observado é uma lorentziana. Este sinal será usado, por meio de um sistema chamado "Lockin Amplifier", que será explicado mais á frente, para travar a frequência do laser no pico da curva. Um esquema da motagem de absorção saturada pode ser visto na figura 6.5

Este tipo de montagem nos permite utilizar o feixe que está no esquema de absorção 


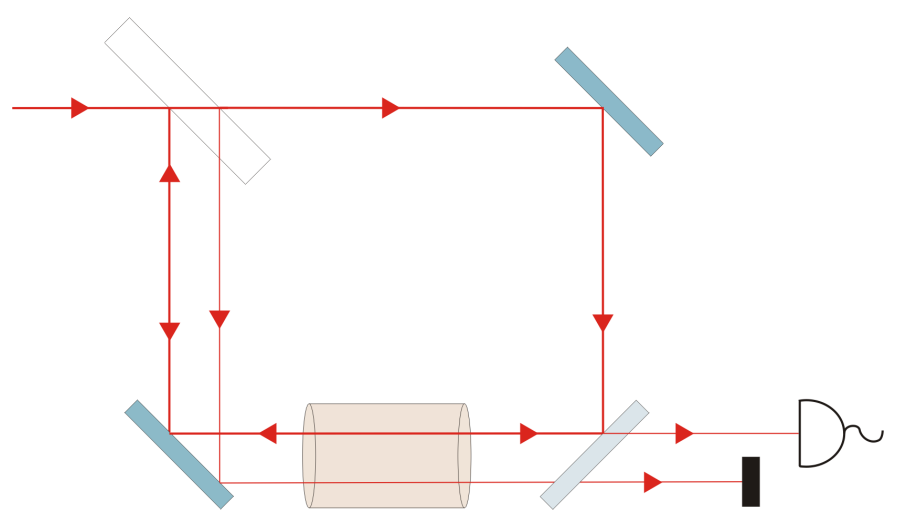

Figura 6.5: Montagem da abosorção saturada.

simples para isolar os picos referentes às transições e cross-over, subtraindo o sinal de absorção simples do sinal de absorção saturada.

\subsection{Laser de Diodo}

A luz utilizada para interagir com os átomos em nosso experimento é um laser de diodo com cavidade externa. Os lasers de diodo livres são dispositivos geralmente baratos e de uso comum em física atômica. Eles são construídos a partir de uma junção p-n em um material semicondutor. A geração de luz no laser ocorre da seguinte forma, uma tensão maior que o gap de energia é aplicada na junção e faz com que os elétrons sofram uma transição da banda de condução para a banda de valência aniquilando as lacunas e emitindo um fóton. Desta forma, um fluxo regular de elétrons origina um fluxo regular de fótons. A junção é feita em uma região que forma um paralelepípedo, como na figura 6.6. As paredes 1, 2, 3 e 4 formam uma guia de onda bidimensional e a face 5 possui um tratamento refletor, atuando como um espelho. A face 6 , devido à diferença entre os índices de refração do semicondutor e do ar, funcionará como um espelho de grande transmitância. Estas 6 faces formarão a cavidade na qual irá ocorrer a oscilação laser.

A oscilação ocorre em uma região com dimensões muito pequenas, além de cada dimensão do plano $x y$ ser uma ordem menor que no eixo $z$. Isto faz com que a cintura do feixe dentro da cavidade seja muito pequena e a divergência muito grande. Assim, o laser deixa a cavidade bastante divergente, com um ângulos típicos da ordem de $30^{\circ}$ no plano $y z$ e $10^{\circ}$ em $x z$. Por isso, uma lente é colocada na saída do feixe para deixá-lo o mais colimado possível ${ }^{4}$.

Outra característica dos lasers de diodo é que estes possuem um ganho muito grande

\footnotetext{
${ }^{4} \mathrm{O}$ laser de diodo, mesmo depois de colimado, possui a secção transversal na forma de uma elipse.
} 


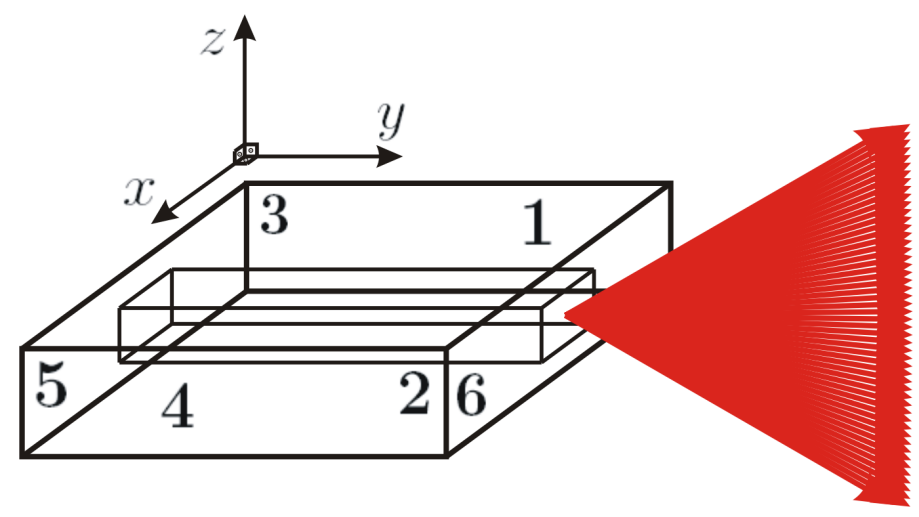

Figura 6.6: Diagrama do laser de diodo livre.

por unidade de comprimento. Por isso, podem oscilar para cavidades com grandes perdas e possuir alto limiar de oscilação. Este fato faz com que a emissão espontânea contribua bastante, o que tem uma influência direta no aumento da largura de linha do laser. Para amenizar estes problemas, usamos o laser com cavidade estendida na configuração Littrow [61], em vez do diodo livre.

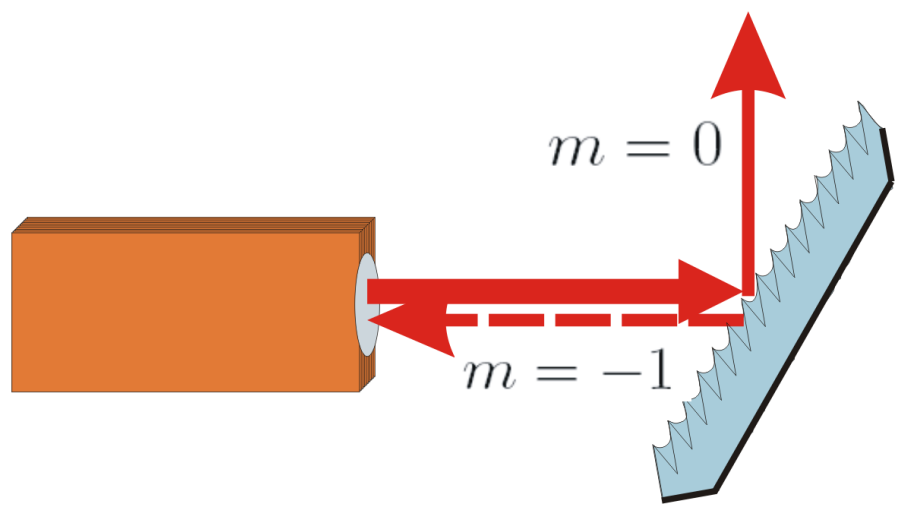

Figura 6.7: Cavidade estendida para o laser de diodo na configuração Littrow.

A cavidade estendida consiste em acoplar o laser livre a uma rede de difração como mostrado em 6.7 A rede fará com que uma parcela dos fótons retorne ao laser aumentanto o tempo de vida dos fótons dentro da cavidade estendida. Utilizamos uma rede de difração holográfica no padrão blaised, que faz com que a ordem -1 de difração seja refletida de volta para o laser e atua como um espelho para a ordem 0. O ângulo de incidência é ajustado de modo que haja o acoplamento com a cavidade, que pode ser verificado observando se mudanças na posição rede de difração afetam o modo laser ${ }^{5}$. O ponto ótimo pode ser encontrado mininizando o limiar de oscilação e fazendo alguns ajustes para correntes maiores. A varredura da cavidade é feita por meio de uma cerâmica piezo-

\footnotetext{
${ }^{5}$ Isto significa que realmente há uma competição entre os modos da cavidade do diodo livre e os da cavidade estendida.
} 
elétrica (PZT), que contrai e dilata em função da tensão aplicada. O PZT é usado para varrer o tamanho da cavidade estendida, o que gera a varredura em frequência. Também é necessário variar a corrente no diodo, pois a linha D2 é bastante extensa e somente o PZT não é capaz de alcançar a amplitude de varredura necessária.

A característica dos lasers de diodo mais importante para este trabalho é o ruído apresentado pelos mesmos. Já foram feitos diversos experimentos que confirmam o excesso ruído de fase presente nestes lasers [62, 20, 19]. E modelos teóricos que levam em consideração este excesso de ruído foram bem sucedidos em explicar dados experimentais de espectroscopia de ruído utilizando lasers de diodo [19, 63]. Para nosso sistema, este ruído de fase clássico se torna um problema, pois pode esconder características quânticas presentes no sistema.

\subsection{Moduladores Acusto-Óticos em Dupla Passagem}

Para realizar deslocamentos de frequência em um feixe laser sem ter que variar a frequência da fonte, podemos empregar diversos métodos, muitos deles descritos em [64]. O método utilizado em nosso experimento será de espalhamento da luz por fônons induzidos em uma rede cristalina por meio de ondas em radiofrequência (RF). Estes dispositivos são chamados de moduladores acusto-ótico (AOM).

As condições de conservação de momento e energia para o espalhamento de um fóton por um fônon são equivalentes a

$$
\begin{aligned}
& \mathbf{k}_{1}=\mathbf{k}_{0}+\mathbf{k}_{f} \\
& \omega_{1}=\omega_{0}+\omega_{f},
\end{aligned}
$$

onde os índices 0,1 e $f$ representam, respectivamente, o campo inicial, final e os fônons. Por isso, além do deslocamento na frequência, há também uma variação da direção de propagação. Caso haja interação de um fóton com mais de um fônon, $\Delta \omega=\omega_{1}-\omega_{0}=m \omega_{f}$

e $\Delta \mathbf{k}=m \mathbf{k}_{f}$, onde $m$ é o número de fônons, e são processos menos prováveis que o espalhamento por um único fônon. O resultado desta interação é equivalente a uma rede de difração, cujas diversas ordens podem ser ajustadas por meio da variação do ângulo de incidência do laser no AOM. Em geral, iremos usar as ordens 1 e -1, mesmo porque elas são mais intensas em relação às outras.

A variação de frequência gerada pelos AOM's é muito pequena comparada à frequência luminosa, que em nosso caso será da ordem de $100 \mathrm{MHz}$. Eles são geralmente usados 
para facilitar a etapa de controle ${ }^{6}$ ou para realizar uma modulação rápida e pequena na frequência do feixe sem perturbar a fonte laser. Tanto a diferença de frequência quanto a amplitude da onda espalhada podem ser controladas por meio do gerador de RF. Este controle pode ser feito à mão, por meio de um botão, ou por meio de uma rampa fornecida, por exemplo, por um gerador de função ${ }^{7}$.

Em nossa montagem utilizamos os moduladores no esquema de dupla passagem, que consiste em fazer o feixe passar por um AOM duas vezes, em sentidos opostos. Esta configuração faz com que o deslocamento em frequência seja dobrado e a perda de potência seja maior, mas o feixe que deixa do AOM após a segunda passagem estará alinhado com o feixe incidente. Este esquema é muito útil quando pretende-se variar a frequência de modulação do AOM durante o experimento, pois esta mudança não afetará o alinhamento como iria ocorrer no caso de passagem simples. Na dupla passagem, um feixe incide no AOM após sair de uma das portas de um cubo polarizador. Os feixes que deixam o AOM passam por uma lente convergente, cujo focos se encontra no centro deste e é refletido de volta por um espelho plano ${ }^{8}$. Entre a lente e o espelho é colocada uma placa de quartode-onda, que, por causa da dupla passagem, irá atuar como uma placa de meia-onda otimizada para girar a polarização do feixe em $90^{\circ}$. Na lâmina, é colocado um anteparo para tampar a ordem 0. O feixe volta para o AOM na mesma direção que o espalhado na ordem 1 e é espalhado novamente na direção do feixe incidente, porém com polarização girada de $90^{\circ}$. Este feixe, então, sairá pela outra porta do cubo polarizador. Uma figura desta montagem pode ser vista na figura 6.8 .

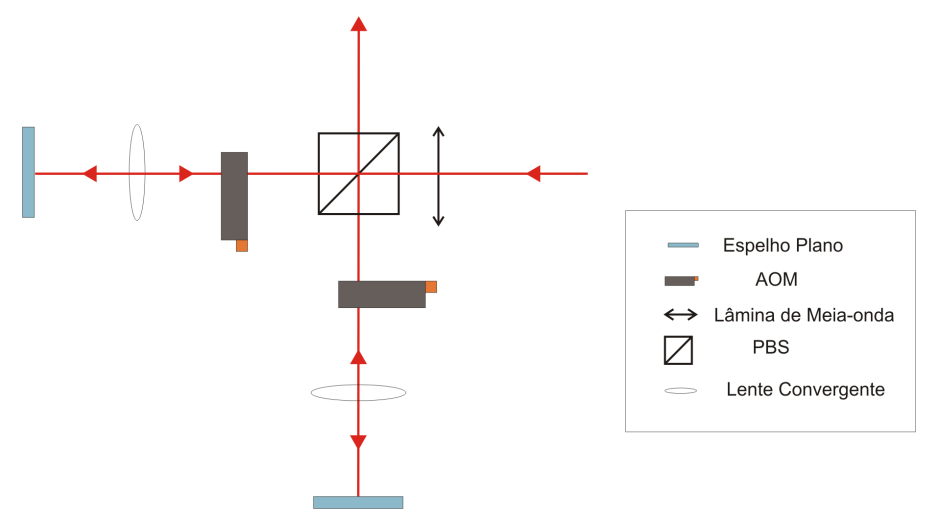

Figura 6.8: Montagem da dupla passagem.

\footnotetext{
${ }^{6}$ Podemos fazer com que a diferença de frequência entre as parcelas do laser enviadas para o controle e para o experimento seja a mesma que entre um pico de cross-over e uma transição atômica. Então, podemos travar o laser em um cross-over, cujo sinal geralmente é maior que o da transição

${ }^{7} \mathrm{O}$ sinal que será enviado ao sistema de Lock-in é obtido por meio de uma rampa jogada em um AOM.

${ }^{8} \mathrm{~A}$ lente e o espleho plano poderiam ser substituídos por um espelho esférico que fizesse o feixe voltar sobre o AOM com o mesmo perfil.
} 


\subsection{Fotodiodos}

Os fotodiodos são dispositivos que emitem um elétron quando um fóton incide em sua superfície. Ele funciona de forma oposta ao diodo laser, um fluxo regular de luz gera uma corrente elétrica regular. Usamos os fotodiodos para realizar as medidas de intensidade de campo. O funcionamento ideal desses fotodiodos consiste na absorção de todos os fótons que incidirem sobre o mesmo, na emissão de um elétron para cada fóton e que essa absorção seja independente da frequência da luz. O sinal do fotodiodo é usado como entrada para um circuito eletrônico que possui duas saídas: A primeira é uma saída DC (direct current) que fornece a corrente média e é medida em um multímetro para efeito de controle, e a segunda é uma saída de alta frequência (HF), que é medida pelo analisador de espectro e fornece as flutuações na corrente elétrica. A corrente medida em ambas as saídas será

$$
\bar{i}_{\gamma}=e \frac{N_{e}}{\Delta t}=e \frac{N_{\gamma}}{\Delta t}=e \bar{n}_{\gamma} \frac{\Delta V}{\Delta t},
$$

onde $e$ é a carga fundamental, $N_{\gamma}$ o número de fótons que acertam a área do detetor no tempo $\Delta t, N_{e}$ é o número de elétrons correspondente e $\bar{n}_{\gamma}$ é a densidade média de fótons.

Para o caso de uma luz monocromática de frequência $\omega, \bar{n}_{\gamma}=\bar{E} /(\hbar \omega \Delta V)$, e a expressão que converte potência luminosa em corrente será

$$
\bar{i}_{\gamma}=\frac{e P_{\omega}}{\hbar \omega}
$$

onde $P_{\omega}=\bar{E} / \Delta t$ é a potência do feixe. Retendo a segunda igualdade de (6.7),

$$
\bar{i}_{\gamma}=e \sum_{\omega, \lambda}\left\langle n_{\omega, \lambda}\right\rangle
$$

para o caso de uma luz não-polarizada e com mais de uma frequência, onde $\hat{n}_{\omega, \lambda}=\hat{a}_{\omega, \lambda}^{\dagger} \hat{a}_{\omega, \lambda}$ é o fluxo de fótons. De modo que a intensidade para um feixe monocromático e polarizado será

$$
I_{\omega}=\frac{\hbar \omega}{A}\left\langle\hat{a}_{\omega}^{\dagger} \hat{a}_{\omega}\right\rangle
$$

onde $A$ é a área do detetor.

\subsection{Deteção Homodina Balanceada}

A deteção homodina é um processo utilizado para medir flutuações da luz. Ela consiste em utilizar um divisor de feixes para misturar feixes de mesma frequência e medir as 
flutuações na soma e na subtração das fotocorrente ${ }^{9}$. Estas medidas fornecerão as flutuações de fase e amplitude do campo eletromagnético. Iniciaremos por mostrar com a luz é transformada por um divisor de feixes. Considere que o ponto de interferência no detetor seja a origem, $e^{i \mathbf{k} \cdot \mathbf{r}}=1$ para todos os modos. Vamos supor os feixes de mesma frequência, de modo que o fator $e^{-i \omega t}$ se torne constante para todos os modos. Podemos ver a interação de um campo com um divisor de feixes como a superposição de dois processos: uma passagem não interagente e um espalhamento na mesma frequência com direção final perpendicular à inicial. Desta forma, é de se esperar que $\hat{c}=t \hat{a}+r \hat{b}$ e $\hat{d}=r^{\prime} \hat{a}+t^{\prime} \hat{b}$, onde $|r|,\left|r^{\prime}\right|,|t|,\left|t^{\prime}\right| \leq 1$.

Impondo a condição de conservação do número de fótons, $\hat{c}^{\dagger} \hat{c}+\hat{d}^{\dagger} \hat{d}=\hat{a}^{\dagger} \hat{a}+\hat{b}^{\dagger} \hat{b}$ e $[\hat{c}, \hat{d}]=0$, podemos mostrar que $|r|=\left|r^{\prime}\right|,|t|=\left|t^{\prime}\right|$ e $|r|^{2}+|t|^{2}=1$. Se escrevemos $r^{\prime}=r e^{i \phi_{r}}$ e $t^{\prime}=t e^{i \phi_{t}}$, temos que $\phi_{r}-\phi_{t}=\pi^{10}$. As fases relativas entre $r$ e $t$, e $r^{\prime}$ e $t^{\prime}$ são arbitrárias e estão associadas á polarização dos feixes que chegam e saem do divisor. Para feixes incidentes linearmente polarizados a transformação para os divisores de feixes será

$$
\left(\begin{array}{c}
\hat{c} \\
\hat{d}
\end{array}\right)=\left(\begin{array}{cc}
t & r \\
-r & t
\end{array}\right)\left(\begin{array}{l}
\hat{a} \\
\hat{b}
\end{array}\right),
$$

onde $r$ e $t$ são reais e a algebra, $\left[\hat{c}, \hat{c}^{\dagger}\right]=\left[\hat{d}, \hat{d}^{\dagger}\right]=1$, é mantida. Os feixes que saem do detetor são medidos em dois fotodiodos, cujos sinais são somados ou subtraídos e enviados ao analisador de espectro.

Em princípio, a interferência pode ser feita usando feixes com fases distintas e diversas calibrações do divisor. A deteção é balanceada quando a transmissão e a reflexão divisor é a mesma. Neste contexto, a expressão (6.9) se simplifica

$$
\begin{aligned}
& \hat{c}=\frac{1}{\sqrt{2}}(\hat{a}+\hat{b}) \\
& \hat{d}=\frac{1}{\sqrt{2}}(\hat{a}-\hat{b}) .
\end{aligned}
$$

Os detetores são sensíveis a qualquer tipo de flutuação. Estas flutuações podem estar presentes no próprio circuito eletrônico ou outras partes do detetor, ou na lus. As flutuações presentes no detetor são medidas sem a presença do feixe e são subtraídas de todas as medidas ${ }^{11}$. As flutuações presentes na luz serão medidas por meio de correlações da soma e subtração das fotocorrentes. Seguindo expressão (6.8), definimos os operadores

\footnotetext{
${ }^{9}$ Podemos utilizar campos com frequências distintas e observar o batimento, esta medida é chamada de deteção heterodina.

${ }^{10}$ Essa defasagem tem o mesmo papel da fase que o feixe clássico ganha ao ser refletido.

${ }^{11}$ Este é o chamado ruído eletrônio ou escuro.
} 
intensidade medidos nos detetores 1 e 2 como

$$
\begin{aligned}
& \hat{I}_{1}=K \hat{d}^{\dagger} \hat{d} \\
& \hat{I}_{2}=K \hat{c}^{\dagger} \hat{c} .
\end{aligned}
$$

Como mediremos soma e subtração dos sinais nos fotodetetores, definimos as intensidades de soma e subtração como

$$
\begin{gathered}
\hat{I}_{+}=\hat{I}_{2}+\hat{I}_{1}=K\left(\hat{a}^{\dagger} \hat{a}+\hat{b}^{\dagger} \hat{b}\right), \\
\hat{I}_{-}=\hat{I}_{2}-\hat{I}_{1}=K\left(\hat{a}^{\dagger} \hat{b}+\hat{b}^{\dagger} \hat{a}\right),
\end{gathered}
$$

onde $K$ é somente uma constante de proporcionalidade, portanto,

$$
\begin{aligned}
& \hat{I}_{+}^{2}=K^{2}\left[\left(\hat{a}^{\dagger}\right)^{2} \hat{a}^{2}+\left(\hat{b}^{\dagger}\right)^{2} \hat{b}^{2}+2 \hat{n}_{a} \hat{n}_{b}+\hat{n}_{a}+\hat{n}_{b}\right], \\
& \hat{I}_{-}^{2}=K^{2}\left[\left(\hat{a}^{\dagger}\right)^{2} \hat{b}^{2}+\left(\hat{b}^{\dagger}\right)^{2} \hat{a}^{2}+2 \hat{n}_{a} \hat{n}_{b}+\hat{n}_{a}+\hat{n}_{b}\right] .
\end{aligned}
$$

Considere $\hat{a}$ como sendo o campo cujas flutuações queremos medir, que temos um controle sobre a intensidade e a fase do campo $b$ e que este esteja em um estado coerente. Então, o estado do sistema pode ser escrito como $|\psi\rangle=\left|\psi_{a}\right\rangle \otimes|\beta\rangle$, em que $\left|\psi_{a}\right\rangle$ representa o estado do sistema $\hat{a}$. Portanto,

$$
\begin{aligned}
\left\langle\hat{I}_{+}\right\rangle & =K\left[\left\langle n_{a}\right\rangle_{a}+|\beta|^{2}\right], \\
\left\langle\hat{I}_{-}\right\rangle & =K\left[\left\langle\hat{a}^{\dagger}\right\rangle_{a} \beta+\langle a\rangle_{a} \beta^{*}\right], \\
\left\langle\hat{I}_{+}^{2}\right\rangle & =K^{2}\left[\left\langle\left(\hat{a}^{\dagger}\right)^{2} \hat{a}^{2}\right\rangle_{a}+|\beta|^{4}+2\left\langle n_{a}\right\rangle_{a}|\beta|^{2}+\left\langle\hat{n}_{a}\right\rangle_{a}+|\beta|^{2}\right], \\
\left\langle\hat{I}_{-}^{2}\right\rangle & =K^{2}\left[\left\langle\left(\hat{a}^{\dagger}\right)^{2}\right\rangle_{a} \beta^{2}+\left\langle\hat{a}^{2}\right\rangle_{a}\left(\beta^{*}\right)^{2}+\left\langle\left(\hat{a}^{\dagger} \hat{a}+\hat{a} \hat{a}^{\dagger}\right)\right\rangle_{a}|\beta|^{2}+\left\langle\hat{n}_{a}\right\rangle_{a}\right],
\end{aligned}
$$

usando o fato que $\beta=|\beta| e^{i \phi}$, onde $\phi$ é a fase média do campo $\hat{b}$,

$$
\begin{aligned}
& \left\langle\hat{I}_{-}\right\rangle=K|\beta|\left\langle X_{\phi}\right\rangle_{a}, \\
& \left\langle\hat{I}_{-}^{2}\right\rangle=K^{2}|\beta|^{2}\left\langle\left(X_{\phi}\right)^{2}\right\rangle_{a}
\end{aligned}
$$

e como $\left(\Delta \hat{I}_{ \pm}\right)^{2}=\left\langle\hat{I}_{ \pm}^{2}\right\rangle-\left\langle\hat{I}_{ \pm}\right\rangle^{2}$, chegamos a

$$
\begin{aligned}
& \left(\Delta \hat{I}_{+}\right)^{2}=\left[K^{2}\left\langle:\left(\delta \hat{n}_{a}\right)^{2}:\right\rangle+K I_{b}+K I_{a}\right] \\
& \left(\Delta \hat{I}_{-}\right)^{2}=K\left[I_{b}\left\langle\left(\delta X_{\phi}\right)^{2}\right\rangle_{a}+I_{a}\right]
\end{aligned}
$$

onde $I_{a}=\left\langle n_{a}\right\rangle_{a}$ e $I_{b}=|\beta|^{2}$ são as intensidades médias. Podemos redefinir o operador $\hat{a} \longrightarrow \hat{a}^{\prime}=\hat{a} e^{i \theta}$, onde $\theta$ é uma fase qualquer, tal que $\left\langle\alpha\left|a e^{i \theta}\right| \alpha\right\rangle \in \mathbb{R}$, com $|\alpha\rangle$ sendo o estado coerente para o modo $\hat{a}$. Portanto, $\theta=-\phi_{a}$, onde $\phi_{a}$ é a fase média do campo $\hat{a}$. Tendo 
isso em mente, podemos interpretar $\phi$ em em (6.17) e (6.16) como a fase relativa entre os campos e não mais como a fase do campo $\hat{b}$, desde que $\hat{a}$, nestas equações, corresponda a $\hat{a}^{\prime}$. O campo representado por $|\beta\rangle$ é chamado de oscilador local e, geralmente, $I_{b} \gg I_{a}$.

Porém, em um detetor real, a eficiência de deteção não é 100\%. Para lidar com as perdas não podemos simplesmente multiplicar um fator $\eta<1$ nas medidas, mas temos que analisar como essas perdas ocorrem. Imagine que incidimos um dado número, $n$, de fótons no detetor e apenas uma quantidade $\eta n$, com $\eta<1$, seja absorvida. Os outros $(1-\eta) n$ fótons passaram sem interagir pela zona do detetor. Mas esse processo é um dos que ocorrem no divisor de feixes, com a diferença que aqui o segundo processo é a absorção em vez do espalhamento. Desta forma, podemos modelar um detetor real por um detetor ideal precedido por um divisor de feixes, como mostrado na figura 6.9 , tal que $\hat{a}=\sqrt{\eta} \hat{f}$.

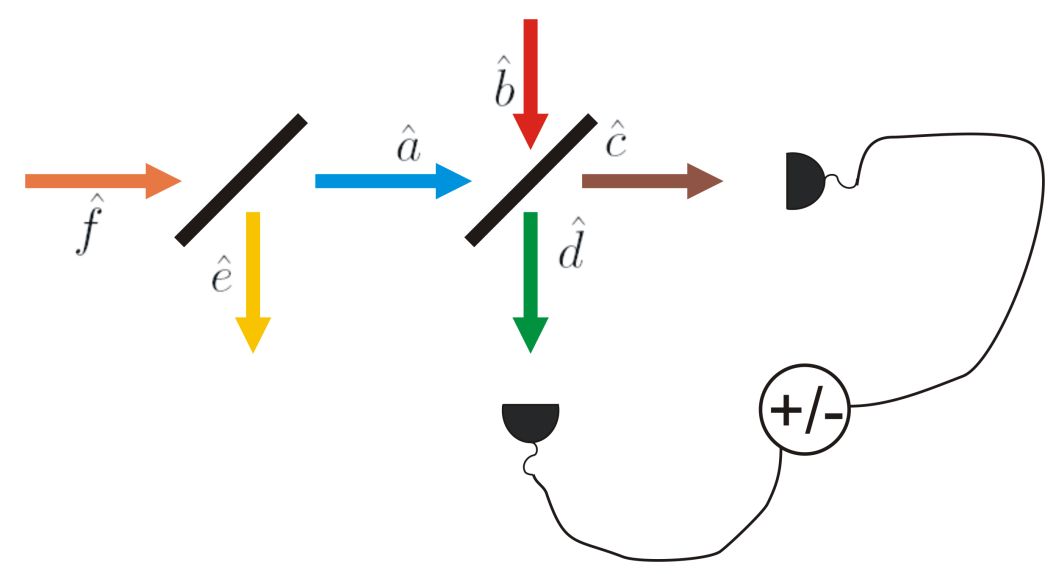

Figura 6.9: Representaçã da deteção homodina com perda por eficiência de deteção. O segundo dividor representa a interferência propriamente dita e o primeiro modela as perdas por eficiência de deteção

Supondo que as perdas são as mesmas para ambos os detetores e substuido $\hat{a}$ nas expressões (6.16) e (6.17) obtemos

$$
\begin{aligned}
\left(\Delta \hat{I}_{+}\right)^{2} & =\left[(\eta K)^{2}\left\langle:\left(\delta \hat{n}_{a}\right)^{2}:\right\rangle_{a}+\eta K I_{b}+\eta K I_{a}\right] \\
\left(\Delta \hat{I}_{-}\right)^{2} & =\eta K\left[I_{b}\left\langle\left(\delta \hat{X}_{\phi}\right)^{2}\right\rangle_{a}+I_{a}\right]
\end{aligned}
$$

Quando o oscilador local satisfaz $I_{b} \gg I_{a}$, podemos desprezar segundo termo em (6.19) e a medida de $\left(\Delta i_{-}\right)^{2}$ é proporcional à flutuação da quadratura $X_{\phi}$. Se $\phi=0$ temos a flutuação na quadratura intensidade e se $\phi=\pi / 2$ obtemos a flutuação na quadratura fase. Essa defasagem pode ser controlada por um PZT colocado no espelho que leva o oscilador local ao divisor de feixes. No caso em que haja apenas o oscilador local, ou seja, 
há vácuo entrando em uma das portas do detetor, a expressão (6.19) se torna

$$
\left(\Delta \hat{I}_{-}\right)^{2}=\eta I_{b}\left\langle\left(\delta \hat{X}_{\phi}\right)^{2}\right\rangle_{\text {vácuo }}=K \eta I_{b}
$$

Este ruído é o chamado shot noise, que significa o ruído obtido pela não continuidade dos portadores de corrente (no caso os elétrons) e depende da raíz quadrada do número de portadores. Este tipo de ruído aparece em diversas situações, como no caso da chuva caindo em uma superfície, onde há uma pressão média mensurável e uma flutuação em torno deste valor pelo fato da chuva ser formada por gotas. Como mostrado na equação anterior, o shot noise medido desta forma é proporcional à flutuação de quadratura no estado de vácuo. Esta flutuação é a mesma para qualquer quadratura e é a mínima permitida pelas relações de incerteza. Note que o shot noise é linear com a intensidade média do oscilador local e, em muitos lugares, já é definido como $K \eta$, ou seja, normalizado pela intensidade média. Todas as medidas que fazemos serão divididas por esta quantidade, de forma que o ruído do vácuo seja igual a 1.

Analisando o caso em que não há oscilador local, $I_{b}=0,\left(\Delta i_{-}\right)^{2}$ é linear com a intensidade média do campo no estado $\left|\psi_{a}\right\rangle$. A inclinação da reta fornece o shot noise normalizado pela intensidade. Desta forma, ainda que não dispomos de um estado coerente para usar como oscilador local, podemos realizar a medida do shot noise com o esquema de deteção balanceada. A medida de soma é o ruído total do laser e será quadrática com a intensidade, comportamento que é um indicador da sanidade do sistema de deteção. Para o sistema $a$ em um estado coerente, $\left\langle:\left(\delta \hat{n}_{a}\right)^{2}:\right\rangle_{a}=0$ e $\left\langle\left(\delta \hat{X}_{\phi}\right)^{2}\right\rangle_{a}=1$, fazendo com que $\left(\Delta \hat{I}_{-}\right)^{2}=\left(\Delta \hat{I}_{+}\right)^{2}$ e ambos os sinais sejam lineares.

As flutuações que medimos podem ter como origem diversos fenômenos e iremos citar os mais importantes. O ruído que nos interessa é o presente a própria luz, que é proporcional ao ruído gerado pelos elétrons que deixam o detetor. Este ruído passa à corrente formada por estes elétrons, a qual é medida no analisador de espectros. Além deste, há o ruído térmico gerado pelos elétrons que fazem funcionar a eletrônica do sistema de aquisição, como o analisador de espectro, chamado de ruído escuro. Este é medido sem a presença da luz e é subtraído de todos os outros, de modo que corresponda ao zero em nossas medidas. Há também outras fontes de ruído, como vibrações na mesa, oscilação na corrente elétrica etc. O detetor não distingue entre qual a origem do ruído, portanto, devemos eliminar todas as fontes de ruído indesejadas para poder obter o sinal mais limpo possível. 


\subsection{Analisador de Espectro}

Pelo fato de ser o aparelho que realiza as medidas dos espectro de ruído que foram calculados nos capítulos anteriores, o funcionamento do analisador de espectro será brevemente descrito nesta seção. Uma explicação mais detalhada pode ser encontrada em $[40,65,60]$. A corrente que chega a cada detetor é separada em duas componentes como já dito anteriormente. A diferença entre a saída DC e HF é que a primeira é a corrente média que chega ao detetor e a segunda é a componente de alta frequência e média nula. A saída HF é enviada ao analisador de espectro, que fará as medidas de correlação no domínio da frequência. Assim como a corrente que é obtida na saída DC, as medidas de correlação no analisador de espectro são feitas por meio de uma média temporal,

$$
\langle X\rangle_{t}=\frac{1}{2 T} \int_{-T}^{T} X(t) d t .
$$

Podemos associar essa média temporal a uma média sobre ensemble por meio da hipótese ergódica,

$$
\langle X\rangle_{t} \equiv\langle X\rangle
$$

O analisador de espectros irá medir as correlações de corrente nas bandas laterais para cada frequência $\omega$. O módulo da corrente é feito pela eletrônica assim como a média temporal, calculada em um intervalo de integração ajustável. Desta forma,

$$
\left\langle i_{ \pm}(\omega) i_{ \pm}(-\omega)\right\rangle \equiv \frac{1}{2 T} \int_{-T}^{T} d \tau\left|i_{ \pm}(\omega ; \tau)\right|^{2}
$$

onde $i$ se refere à corrente $\operatorname{HF}$ e $i_{ \pm}(\omega ; \tau)^{*}=i_{ \pm}(-\omega ; \tau)$. Um esquema do circuito no analisardor está mostrado na Figura 6.10.

A primeiro estapa deste diagrama de blocos é um amplificador que gera um ganho no sinal de entrada. Um circuito misturador, faz o batimento da frequência de entrada com uma frequência intermediária, para a qual a eletrônica do analisador está otimizada. A frequência de análise pode ser ajustada por meio de um VCO (Voltage Controlled Oscillator), que fará o papel do oscilador local, de forma análoga á deteção homodina. Uma varredura nesta frequência pode ser gerada, fazendo com que as medidas sejam feitas para uma faixa selecinada de frequências. A segunda etapa consiste de um filtro passafaixa com largura ajustável. Fisicamente, a largura desta faixa é a precisão em frequência da medida feita pelo analisador. Ela é chamada de RBW (Resolution Bandwidth) e pode ser ajustada por usuário. 


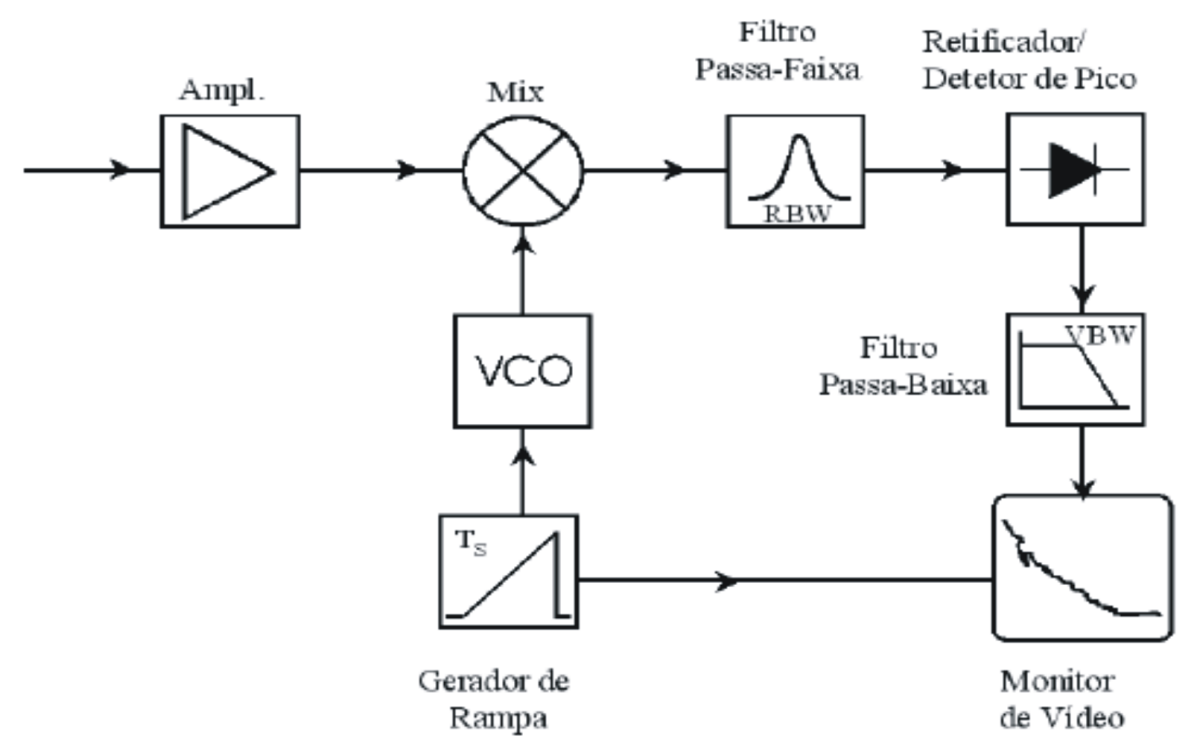

Figura 6.10: Esquema simplificado do circuito interno de um analisador de espectro.

Na terceira etapa, o sinal passa por um diodo retificador, que tem como saída o módulo do sinal de entrada. A última etapa é um circuito integrador e um filtro passa-baixa, na qual será calculada a média temporal do módulo da corrente. O tempo de integração é definido nesta última etapa como o inverso do VBW (Video Bandwidth) e, para que a média faça alguma diferença no sinal que vai para o vídeo, este deve ser menor que o RBW. Na prática, a proporção entre o RBW e o VBW será uma medida direta da suavização da curva obtida. Quanto maior esta proporção, mas suavizada será a curva mostrada na tela do analisador, O intervalo de integração será o inverso do RBW. Caso esta seja menor ou igual a 1, não haverá integração no sinal de correlação, ou seja, a curva obtida na tela será a saída do diodo retificador.

Queremos medir o espectro de ruído em uma banda lateral determinada. Para isso, escolhemos a variação da frequência de análise nula (medida a zero span), que equivale a manter a tensão fixa no VCO. Variamos os parâmetros físicos ${ }^{12}$ e ajustamos o trigger para disparar a medida simultaneamente com a variação destes parâmetros. Outra importante média realizada pelo analisador, é a média sobre o vídeo (BW). Esta média é feita em com uma constante de tempo da ordem de milissegundos (Três ordens de grandeza superior à definida pelo RBW). Utilizamos o BW como forma de suavizar ainda mais a curva por cancelar os efeitos de flutuações lentas não capturadas pelo circuito integrador.

\footnotetext{
${ }^{12}$ Em nosso caso o campo magnético.
} 


\subsection{Sistema de Travamento da Frequência dos Lasers}

O travamento do laser no pico de ressonância é feito por um sistema de Lock-in. Este sistema consiste de uma etapa de diferenciação e outra de ganho controlado por um sinal de erro. O sinal de erro de uma função $F(x)$ é uma curva linear, $\operatorname{Er}_{F}(x)$, que tem alguma relação direta com $F(x)$. Ele tembém deve cruzar o eixo $x$ no ponto em que se deseja travar a função $F$. Por exemplo, se queremos travar uma função senoidal em $x_{0}=0$, a própria função será o sinal de erro, pois é localmente linear e $\sin (0)=0$.

Em nosso caso, estamos interessados em travar a frequência do laser em um pico de absorção. Podemos variar a fequência e medir diretamente a intensidade. Após passar por um esquema de absorção saturada, a intensidade da luz medida apresentará picos nas ressonâncias atômicas e nos cross-overs. Próximo a um máximo, qualquer curva é localmente quadrática, portanto, sua derivada será localmente linear. Sendo assim, o sinal de erro mais natural a ser utilizado, é a derivada do sinal de absorção. Esta derivada é feita pela eletrônica da seguinte forma, ajustamos a frequência do laser para o máximo de intensidade e geramos uma pequena modulação na mesma, tal que $\epsilon \ll \omega_{0}$, com $\omega_{0}$ sendo a frequência do pico. Então podemos expandir $I(\omega)$ próximo de $\omega_{0}$ como

$$
I\left(\omega_{0}+\epsilon\right) \approx I\left(\omega_{0}\right)+\frac{\partial I}{\partial \omega_{0}} \epsilon .
$$

Desta forma, a diferença de intensidades é localmente proporcional ao sinal de derivada e o circuito eletrônico realiza a subtração dos sinais. Esta etapa diferenciadora do processo somente é necessária caso se queira travar em um pico. Para o travamento na na meia altura, a própria absorção seria utilizada como sinal de erro.

Após obtido, o sinal de erro é enviado à etapa de ganho. Esta etapa modifica a tensão no controle da frequência de modo a fazer o sistema voltar para ao ponto com sinal de erro nulo. O valor desta tensão é proporcional ao sinal de erro, ou seja, caso este seja positivo o circuito fornecerá uma tensão que fará ele reduzir e vice-versa. Como o valor da tensão é proporcional ao módulo do sinal de erro, o processo será convergente e funcionará como um processo de equilibrio dinâmico. 


\section{Descrição do Aparato Experimental e Resultados Obtidos}

Neste capítulo faremos a descrição do aparato experimental utilizado para realizar as medidas de ruído propostas e apresentaremos os resultados obtidos.

\subsection{Aparato Experimental}

Um diagrama esquemático do aparato experimental é descrito na Figura 7.1. O diodo laser utilizado é um Sanyo, focalizado por uma lente Newport. Após sair da cavidade, o feixe passa por um isolador ótico, que tem como objetivo evitar que alguma parcela do feixe retorne ao laser e de origem a modos indesejados na cavidade, e é dividido em um cubo polarizador. Uma parte do feixe transmitida pelo cubo vai para o experimento passando por um prisma anamorfo, que faz a seção transversal do laser ficar mais próximo de um círculo.

A parte do feixe refletida pelo cubo vai para a etapa de controle, que cosiste em duas duplas passagens e uma espectroscopia de absoção saturada. As duplas são montadas de modo que o feixe que sai de uma delas é usado como feixe incidente para a outra. Um dos moduladores é otimizado para a ordem 1 e o outro para -1, o que fará com que, após deixar o segundo AOM, o laser possua a frequência de

$$
\Delta f_{L}=2\left(f_{1}-f_{2}\right)
$$

onde $f_{L}$ e $f_{i}$ são, respectivamente, as frequências do laser e de espalhamento de cada AOM. Desta forma, podemos travar o feixe que vai para o controle em uma frequência próxima da transição atômica e buscar a melhor posição com o controle de um dos AOM's. A modulação em frequência para o Lock-in é feita no outro AOM. Ambos os AOM's possuem a frequência central de $80 M h z$ e podem ser variados de $20 M h z$ para mais ou 
menos.

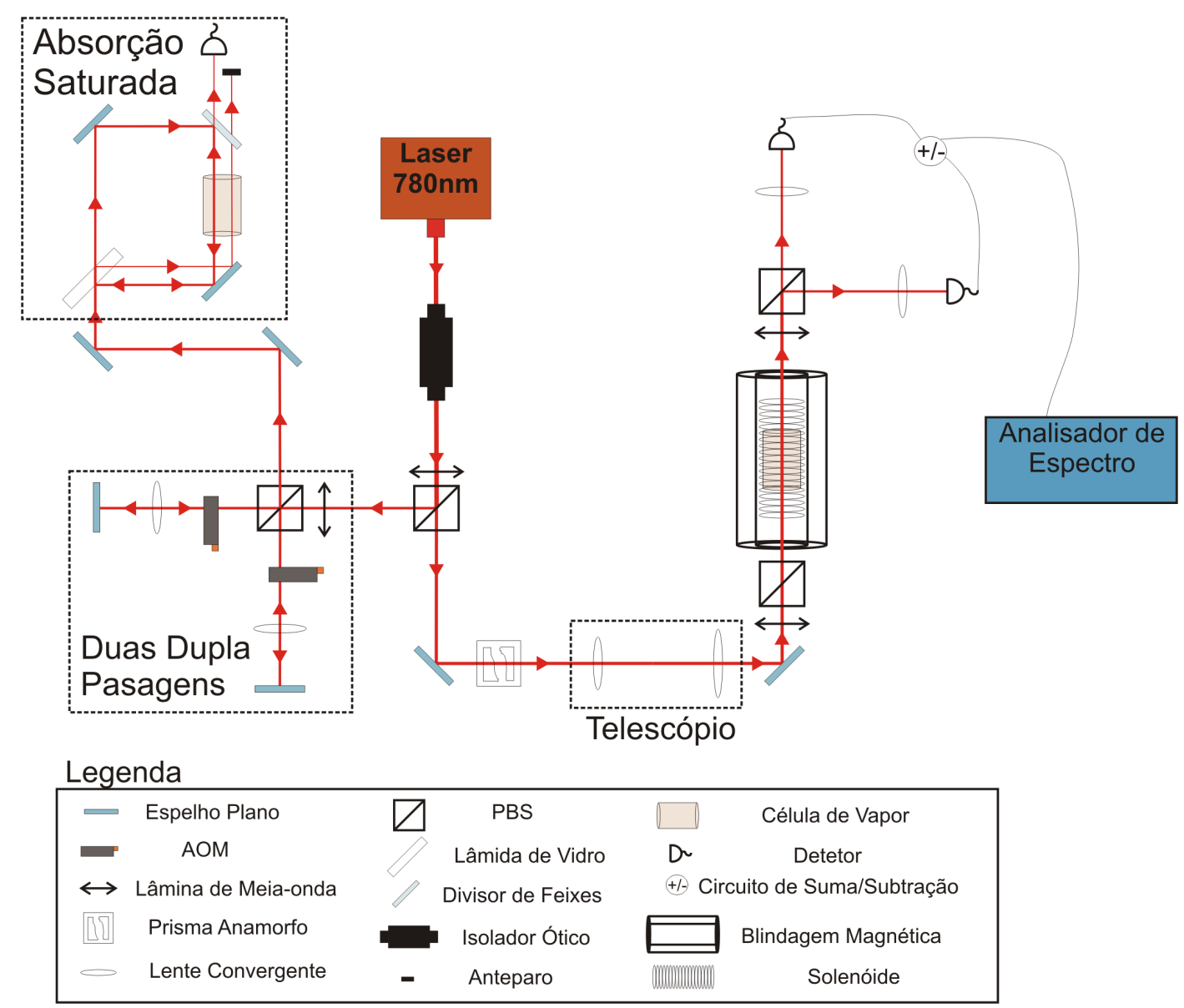

Figura 7.1: Montagem Experimental

Depois das duplas passagens o laser vai para a absorção saturada. Primeiramente, ele incide sobre uma lâmina de vidro grossa, cujas faces refletem dois feixes paralelos de pouca intensidade e deixa passar a maior parcela feixe incidente, que vai servir como o feixe intenso de saturação. Ambos os feixes pouco intensos passam por uma célula auxiliar e o feixe intenso é superposto ao feixe que será detetado por um divisor de feixes.

\subsubsection{Campo Magnético e Célula de Vapor}

Geramos o campo magnético por meio de um solenóide de 9 voltas $/ \mathrm{cm}$. A corrente é fornecida por um gerador de função acoplado a um buffer de corrente, um dispositivo eletrônico que faz com que a impedância de saída seja maior que a de entrada. O ganho em tensão, em nosso caso, é unitário, o que faz com que o buffer funcione como uma fonte de corrente.

A célular de vapor cilindrica é colocada dentro do solenóide de modo que a direção 
do campo magnético coincida com o eixo de simetria da célula. A blindagem magnética é feita por uma cobertura dupla de $\mu$-metal ${ }^{1}$, permitindo que apenas o campo magnético gerado pelo solenóide afete os átomos. Como a luz se propaga na direção paralela ao campo magnético, sua polarização terá apenas componentes no plano transverso.

\subsection{Medidas do Shot Noise e Ruído de Subtração}

Calibramos o shot noise por meio de um esquema de deteçao balanceada como mostrado na figura 7.3. Esta medida é feita desligando o campo magnético e colocano os átomos fora de ressonância, de modo que a absorção seja desprezível. Caso o laser fosse um estado coerente os sinais de soma e subtração seriam os mesmos, pois $\left\langle:\left(\delta \hat{n}_{a}\right)^{2}:\right\rangle=0$ e $\left\langle\left(\delta \hat{X}_{\phi}\right)^{2}\right\rangle_{a}=1$. Em nosso caso, $\left(\Delta \hat{I}_{+}\right)^{2}$ é quadrático com a intensidade e fornece a medida do ruído total do laser. O gráfico 7.2 mostra os ruídos de soma e subtração fora de ressonância em função da medida DC nos detetores.

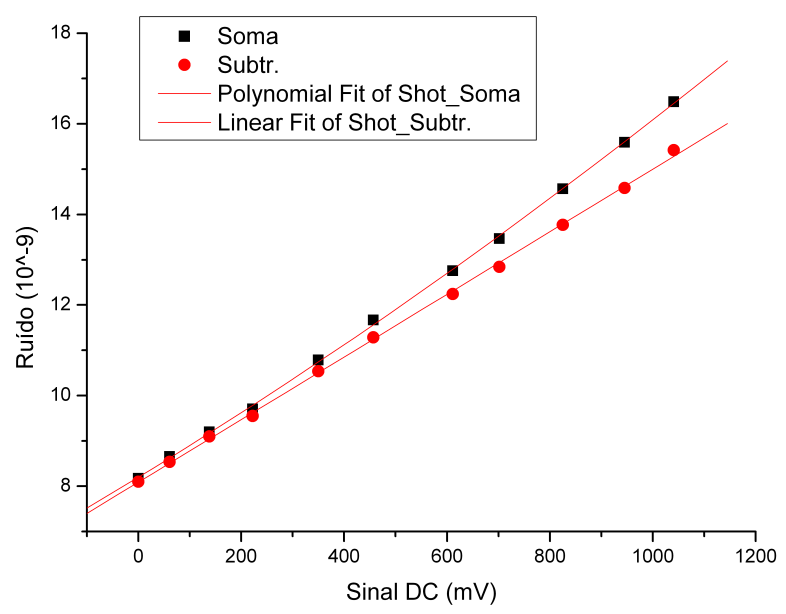

Figura 7.2: Ruído de soma e subtração fora de ressonância em função da soma da voltagem média nos dois detetores.

A homodinagem é feita com o vácuo que entra pelo PBS que está situado antes da célula. A polarização deste vácuo é perpendicular à feixe incidente e é girada pela lâmina de onda situada após a célula de modo que haja vácuo na mesma polarização de cada feixe que sai do segundo PBS. O vácuo que entra pela porta vazia do segundo PBS não interfere com o feixe laser, pois está polarizado na direção perpendicular à do mesmo.

As montagens feitas anteriormente no laboratório realizavam a homodinagem de forma um pouco diferente. Nelas, eram utilizados três PBS após a interação com os átomos para

\footnotetext{
${ }^{1}$ Este material possui permissividade magnética de $\mu_{m}=10^{4}$ a $10^{6} \mathrm{~T} . \mathrm{m} / \mathrm{A}$.
} 


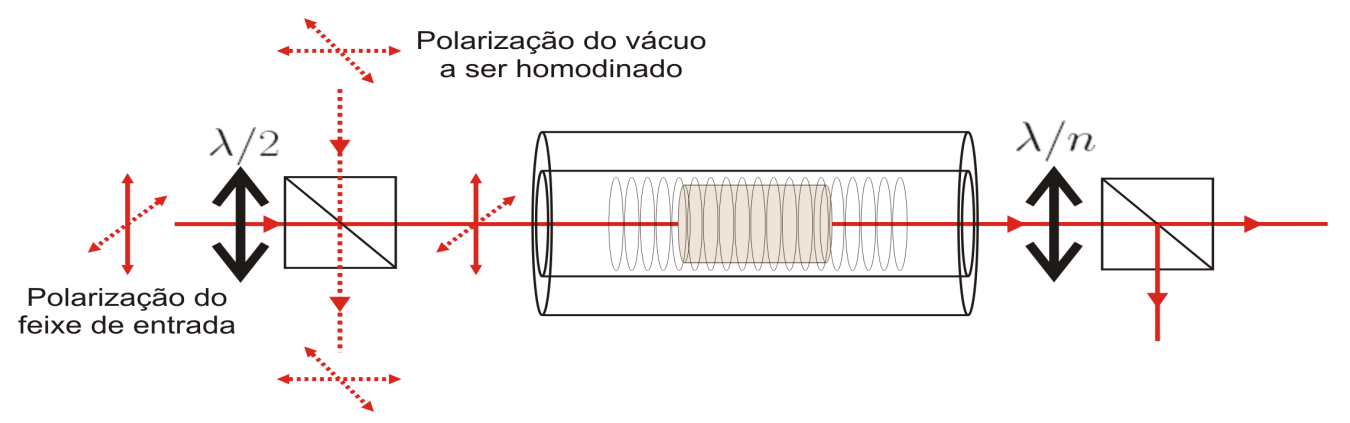

Figura 7.3: Diagrama da interação que mostra a entrada do feixe incidente na célula de vapor. O vácuo que entra na direção perpendicular é responsável pela homodinagem.

que os feixes pudéssem ser misturados com o vácuo nos PBS [19]. Na montagem atual, o vácuo com o qual o feixe é homodinado, também é modificado pela interação com os átomos, assim como o modo laser. Esta diferença pode ser responsável por eventuais discrepâncias entre estas medidas e as anteriores, e será analisada de forma mais direta.

\subsection{Ruído de Soma e Subtração e Coeficiente de Cor- relação}

A teoria desenvolvida nos capítulos 3 e 5 nos permitiu calcular os espectros de ruído para a correlação entre os feixes que chegam aos detetores. Entretanto, o que medidos diretamente é o espectro de ruído de soma e subtração. Nas medidas de soma e subtração feitas na deteção balanceda, primeiro somamos ou subtraimos as fotocorrentes, depois elas são enviadas ao analisador de espectros. O operador intensidade é $\hat{I}_{ \pm}(t)=\hat{E}_{ \pm}^{(-)}(t) \hat{E}_{ \pm}^{(+)}(t)$, portanto, a função de correlação de intensidade será

$$
\begin{aligned}
C_{ \pm}\left(t, t^{\prime}\right) & =\left\langle\hat{I}_{ \pm}(t) \hat{I}_{ \pm}\left(t^{\prime}\right)\right\rangle \\
& =C_{\mu^{\prime}, \mu^{\prime}}^{(2)}\left(t, t^{\prime}\right)+C_{\mu, \mu}^{(2)}\left(t, t^{\prime}\right) \pm C_{\mu^{\prime}, \mu}^{(2)}\left(t, t^{\prime}\right) \pm C_{\mu, \mu^{\prime}}^{(2)}\left(t, t^{\prime}\right)
\end{aligned}
$$

com $C_{\mu, \mu^{\prime}}^{(2)}\left(t, t^{\prime}\right)$ definida em (2.84). Ao substituir os operadores pelos valores médios e flutuações, obteremos para a segunda ordem nas flutuações,

$$
S_{ \pm}(\omega)=S_{\mu^{\prime}, \mu^{\prime}}(\omega)+S_{\mu, \mu}(\omega) \pm S_{\mu^{\prime}, \mu}(\omega) \pm S_{\mu, \mu^{\prime}}(\omega)
$$

Os gráficos de soma e subtração, considerando $\langle\hat{q}(\omega) \hat{q}(-\omega)\rangle=1$, devido à contribuição somente da autocorrelação de fase são mostrados na figura 7.4. O primeiro gráfico é referente à transição $F=1 \longrightarrow F^{\prime}=0$ e o segundo à $F=2 \longrightarrow F^{\prime}=1$, as transições mais simples em que ocorre EIT neste tipo de sistema. 

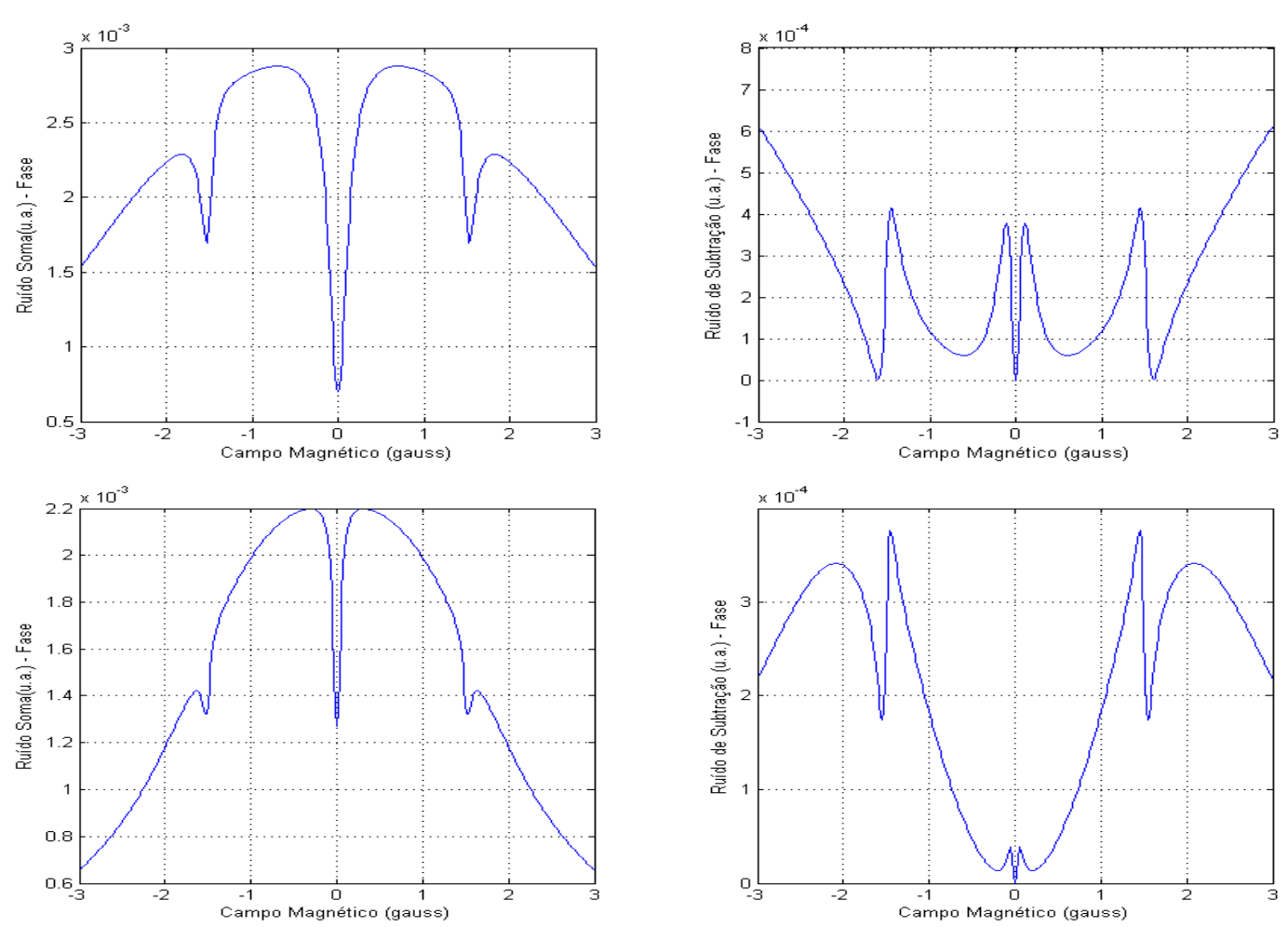

Figura 7.4: Curva teórica do ruído de amplitude do feixe transmitido. Estas curvas mostram apenas a contribuição do ruído de fase do feixe incidente. As de cima são para a transição $F=1 \longrightarrow F^{\prime}=0$ e as de baixo para $F=2 \longrightarrow F^{\prime}=1$.

Se definimos o espectro de ruído simetrizado como $2 S_{\mu^{\prime}, \mu}^{s}(\omega)=S_{\mu^{\prime}, \mu}(\omega)+S_{\mu, \mu^{\prime}}(\omega)$, tal que $S_{\mu^{\prime}, \mu}^{s}(\omega)=S_{\mu, \mu^{\prime}}^{s}(\omega)$, portanto,

$$
S_{\mu^{\prime}, \mu}^{s}(\omega)=\frac{1}{4}\left[S_{+}(\omega)-S_{-}(\omega)\right]
$$

que é a e correlação entre os feixes mostrada no gráfico 3.5 para um sistema de dois níveis e é obtido diretamente das medidas de soma e subtração. O coeficiente de correlação normalizado é dado por [41]

$$
C_{\mu^{\prime}, \mu}(\omega)=\frac{S_{\mu^{\prime}, \mu}^{s}(\omega)}{\sqrt{S_{\mu, \mu}(\omega) S_{\mu^{\prime}, \mu^{\prime}}(\omega)}} .
$$

que tem a propriedade de que $\left|C_{\mu^{\prime}, \mu}(\omega)\right| \leq 1$. Este pode ser comparado diretamente com o que ocorreria caso a luz estivesse em um estado coerente, que possui ruído de soma igual ao se subtração, fazendo a correlação se anular. Interpretamos a correlação nula como no caso em que os campos estão totalmente descorrelacionados. Seria este o caso em que a separação é feita pelo divisor de feixes de forma totalmente aleatória, como seria para um estado coerente.

O processo que ocorre na EIT é a inibição da absorção de um campo devido à precença 
do outro próximo à ressonância. Idealmente, isto implica em correlação entre os feixes, pois a presença de cada fóton do bombeio inibirá a absorção de um fóton do feixe sonda. Neste caso, o sinal de soma será maior que o sinal de subtração, indicando que na mesma frequência as flutuações temporais possuem, para máxima correlação, o mesmo valor. Ainda na condição de ressonância Raman, mas fora da ressonância em cada transição também pode ser osbservada a EIT, mas ocorre também transições Raman de dois fótons. Neste processo, um fóton é absorvido do bombeio e emitido no sonda e vice-versa, gerando anticorrelação entre os feixes. Pensando em termos de correlações temporais, a preseça de um fóton em um dos detetores está condicionada ao não aparecimento de um fóton no outro detetor. Esta competição entre os processos de EIT e o espalhamento Raman possivelmente gera as regiões de correlação e anticorrelação observadas.

As medidas apresentadas foram feitas com a frequência do laser travada em um pico de cross-over das transições partindo do estado fundamental $F=2$. Como cada átomo percebe o laser com uma frequência diferente, o AOM foi ajustado de modo a dimunuir o efeito deste alargamento para o sinal de ruído. Isto foi feito escolhendo uma frequência para o AOM, tal que os vales no sinal de soma à potência máxima sejam minimizados. Como o espaçamento entre os níveis excitados é menor que a largura Doppler, o sinal será uma soma sobre as contribuições dos estados excitados $F^{\prime}=1,2,3$. Porém, pelo fato de o estado com $F^{\prime}=1$ poder decair apenas para $F=2$, a contribuição do mesmo para o sinal de ruído será maior e o sinal observado será típico de EIT.

\subsection{Medidas com Lâminas de Meia-Onda}

O feixe incide na amostra atômica com polarização linear e vertical. Na nossa montagem, a separação para a deteção balanceada é feita por meio de um cubo polarizador. Caso o feixe passasse pelo cubo diretamente após sair da célula, todo ele seria transmitido. Levando isso em consideração, colocamos uma lâmina de meia-onda antes do cubo para girar a polarização do feixe em $45^{\circ}$, de modo a dividir igualmente o feixe. A polarização que chega nos detetores é horizontal para um deles e vertical para o outro. Porém, a informação contida nestas polarizações se refere às componentes $+45^{\circ} \mathrm{e}-45^{\circ}$ que interagiram com os átomos ${ }^{2}$. Fizemos diversas medidas e os gráficos apresentados serão da melhor delas. As medidas são feitas para potências do feixe incidente distintas e em cada uma delas variamos o campo magnético.

\footnotetext{
${ }^{2}$ A lâmina de onda gira cada uma destas componentes de $45^{\circ}$, tal que se tornem verticais e horizontais.
} 


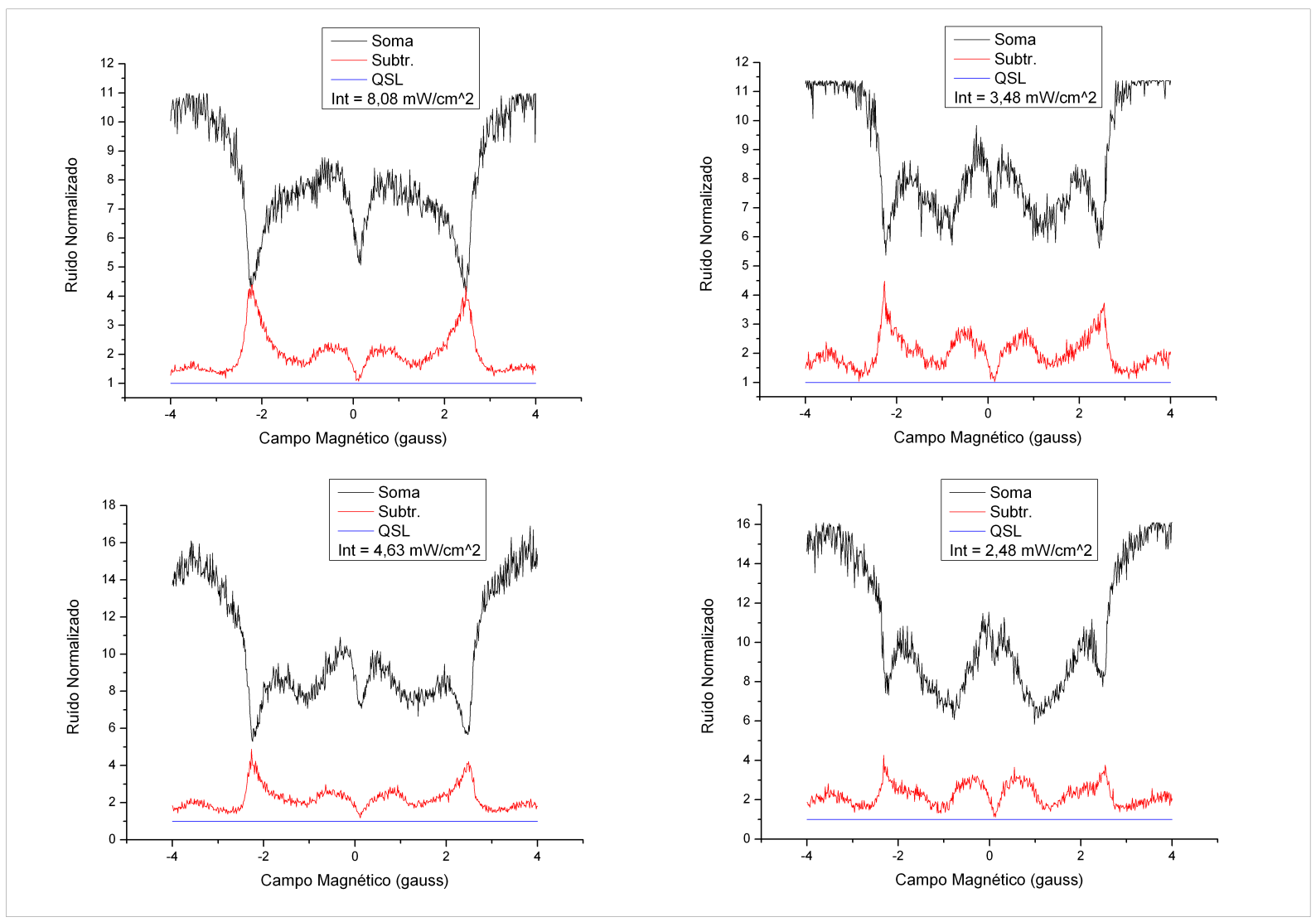

Figura 7.5: Espectros de Ruído de Soma de Subtração para Diferentes Intensidades

Os parâmetros do analisador de espectro são $R B W=100 k \mathrm{~Hz}, V B W=10 k \mathrm{~Hz} \mathrm{e}$ frequência de análise $\omega=3.0 \mathrm{MHz}$. O ruído eletrônico já foi subtraído, correspondendo ao zero na escala, e o 1 representa o limite quântico padrão (no inglês SQL). Vemos que nenhuma das quadraturas cruza o limite quântico, portanto nenhum efeito de compressão de ruído ou geração de estados não-clássicos foi obtido. O ruído de subtração apresenta picos laterais que aparecem quando $B=\omega / 2 \gamma_{b}$ e a estrutura central em forma de $M$, que já foi observada em medidas anteriores [20, 19], característica da conversão de ruído de fase em ruído de amplitude.

O sinal de soma varia com a intensidade como mostra o gráfico 7.8. A forma de tridente invertido, que caracteriza o sinal em altas itensidades, muda para baixas intensidades tomando a forma de um pico central e pequenos picos laterais. Essa inversão de picos foi observada em duas baterias de medidas, mas ainda não sabemos com certeza a origem da mesma. A causa mais provável é a influência das flutuações de amplitude que se tornam consideráveis á medida que a potência do feixe incidente diminiu. O caráter super poissoniano das flutuações de intensidade do laser é mostrado pela parábola na figura 7.2. Para baixas intensidades, este sinal pode ser aproximado por uma reta e se torna muito 

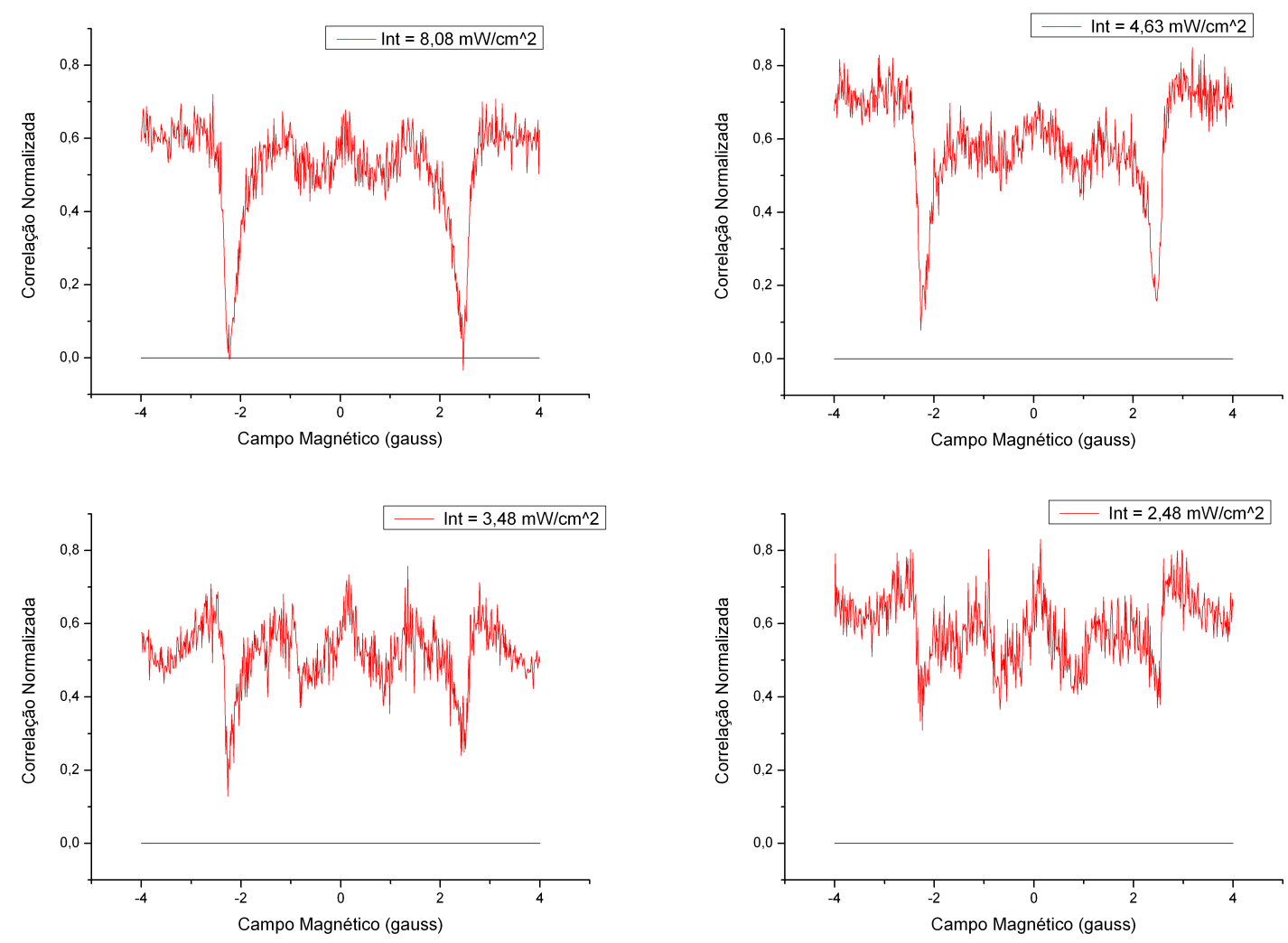

Figura 7.6: Correlações Normalizadas com a Separação feita por uma lâmina de $\lambda / 2$.

próximo do shot noise. Nestas condições, o laser de diodo se torna próximo de um estado coerente na quadratura amplitude. Um comportamento deste tipo também é esperado para a quadratura fase, porém não chegamos a fazer uma caracterização direta do ruído de fase do laser. Na figura 7.7 mostramos a contribuição para o ruído de soma e subtração devido à autocorrelação de amplitude também referente às transições $F=1 \longrightarrow F^{\prime}=0$ e $F=2 \longrightarrow F^{\prime}=1$ fazendo $\langle\hat{p}(\omega) \hat{p}(-\omega)\rangle=1$.

Como pode ser visto nos gráficos 7.7 e 7.4, a contribuição para o ruído de soma devido à autocorrelação de amplitude é 3 ordens de grandeza maior que a contribuição devido à autocorrelação de fase caso as mesmas sejam iguais. Entretanto, como o ruído de fase do laser de diodo é bem maior que o ruído de amplitude, a contribuição daquele para o sinal de ruído compensa esta diferença e faz com que, para altas intensidades, o sinal de soma seja fruto somente do ruído de fase presente no feixe incidente. Para baixas intensidades, o ruído total do laser diminui e a contribuição do ruído de amplitude começa a se tornar mais significativa. Porém, apesar da inversão ser clara, a potência onde a mesma ocorre é da ordem de $200 \mu W$ no detetor. A esta potência o sinal do detetor já não é mais tão confiável, pois o ruído eletrônico fica muito próximo do shot noise. Para contornar este 

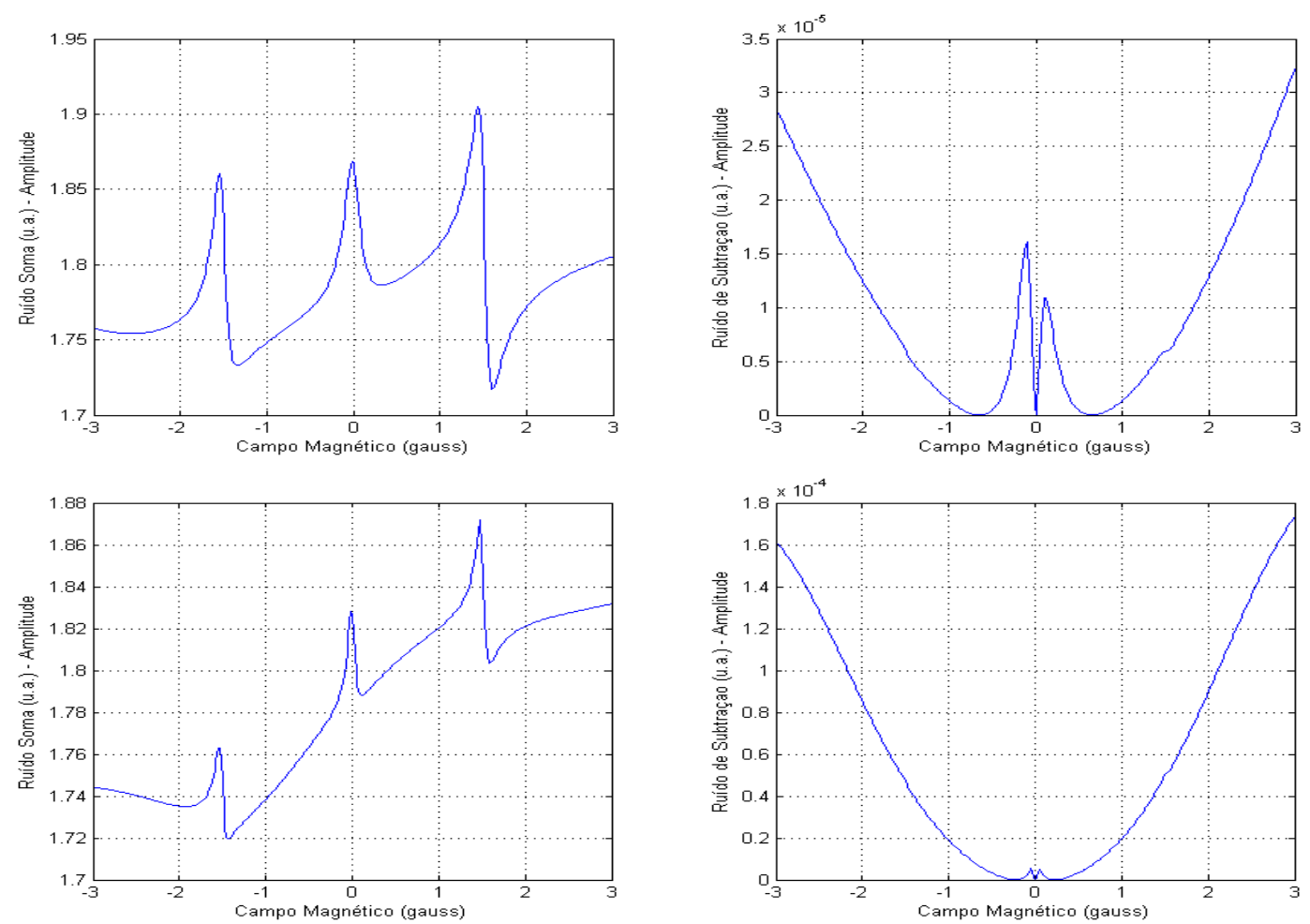

Figura 7.7: Curva teórica do ruído de amplitude do feixe transmitido. Estas curvas mostram a contribuição do ruído de amplitude do feixe incidente. As de cima são para a transição $F=1 \longrightarrow F^{\prime}=0$ e as de baixo para $F=2 \longrightarrow F^{\prime}=1$.

problema, detetores mais sensíveis estão sendo desenvolvidos, mesmo por que é nesta faixa de potências que se espera observar compressão de ruído.

Nos gráficos 7.6 e 7.8 vemos que, para alta potência, as componentes $+45^{\circ}$ e $-45^{\circ}$ chegam a ser descorrelacionadas nos picos laterais. Para todos os valores de potência de campo magnético, há somente correlação entre estas componentes. Como as polarizações analisadas não são diretamente os feixes bombeio de sonda, não podemos afirmar categoricamente que o processo de EIT predomine sobre as transições Raman na geração de correlação entre os feixes. As medidas com lâminas de $\lambda / 4$ serão mais claras neste sentido

\subsection{Medidas com Lâminas de Quarto-de-Onda}

Podemos balancear os detetores usando lâminas de quarto-de-onda também. Estas, quando otimizadas, irão fazer o feixe com polarização linear que sai da célula se torne circularmente polarizado, balancendo os detetores.

A polarização linear pode ser decomposta em componentes circulares girando em sentidos opostos. A lâmina de onda atuando nas componentes circulares irá realizar o 

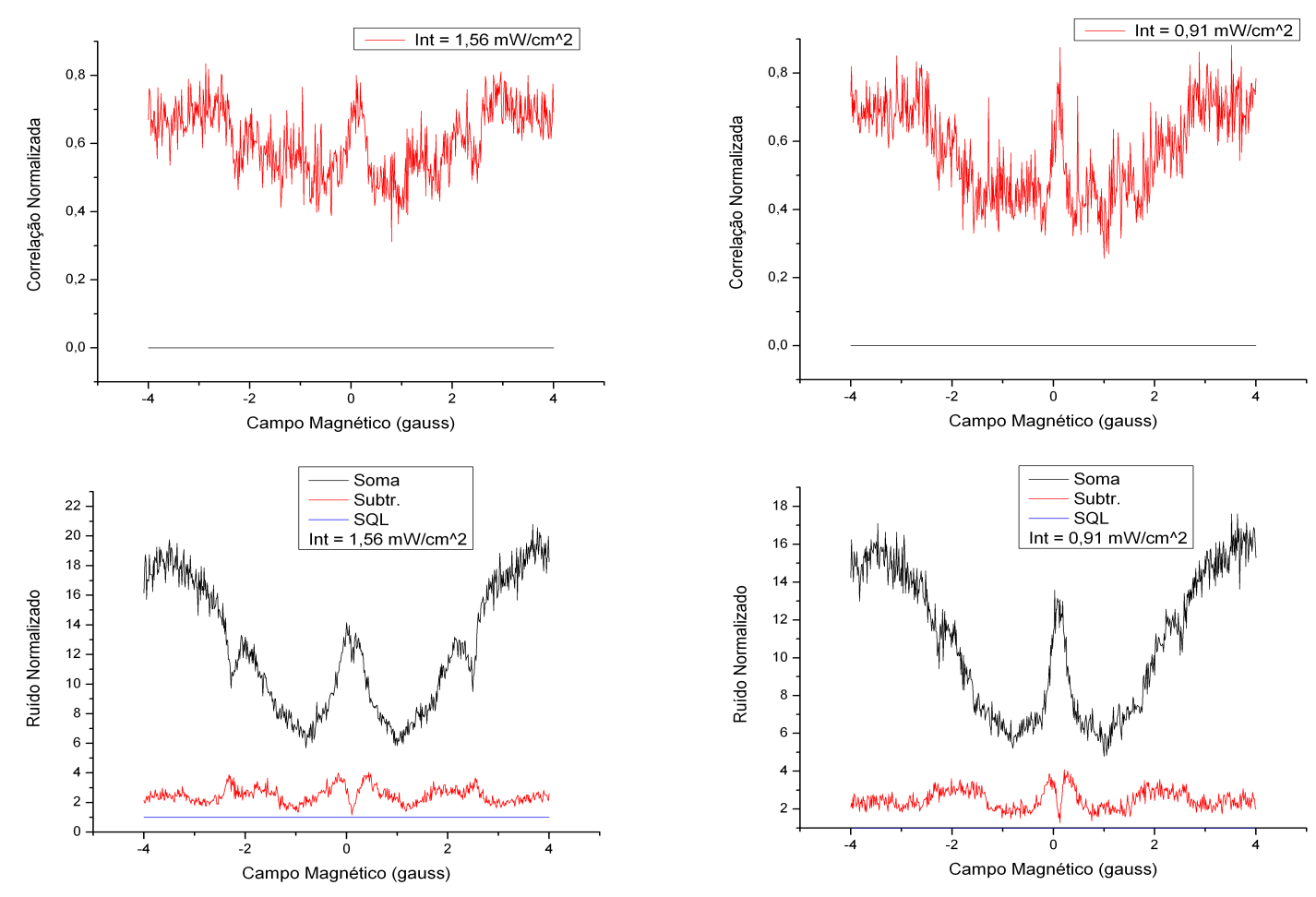

Figura 7.8: Correlações e espectros de ruído e para baixas intensidades com a separação feita por uma lâmina de $\lambda / 2$.

processo inverso, ou seja, transformá-las em componentes lineares. Desta forma, fazer o balanceamento com lâminas de quarto-de-onda significa separar as componentes $\sigma^{+}$e $\sigma^{-}$do feixe laser. Esta separação é a mais intuitiva para o estudos das correlações, pois realmente estamos separando os feixes de sonda e bombeio. Com a lâmina de meia-onda estávamos enviando para cada detetor uma combinação de sonda e bombeio.

Estas medidas foram feitas também com $\omega=3.0 \mathrm{MHz}$ e $R B W=100 \mathrm{kHz}$, mas com $V B W=1 k H z$. O sinal de subtração apresenta os picos laterais e a mesma estrutura central em forma de $M$ que caracteriza a conversão de ruído de fase em ruído de amplitude pelos átomos, porém mais acentuada. Observamos um estreitamento desta estrutura a baixas potências, como era de se esperar. Para a separação com $\lambda / 2$ também ocorre o estreitamento, mas este é menos evidente que no caso com $\lambda / 4$. Também podemos observar a mesma inversão e picos no sinal de soma, mesmo por que este é independente da forma como a luz é separada. A diferença entre as separações em linear e circular aparece apenas no sinal de subtração e, por este apresentar picos menores para a separação feita com $\lambda / 2$, a inversão de picos no sinal de soma fica mais evidente no primeiro caso.

Para a separação do $\lambda / 4$ observamos passagens de correlação para anticorrelação e vice-versa à medida que variamos o campo magnético. Esta passagem ocorre para qual- 

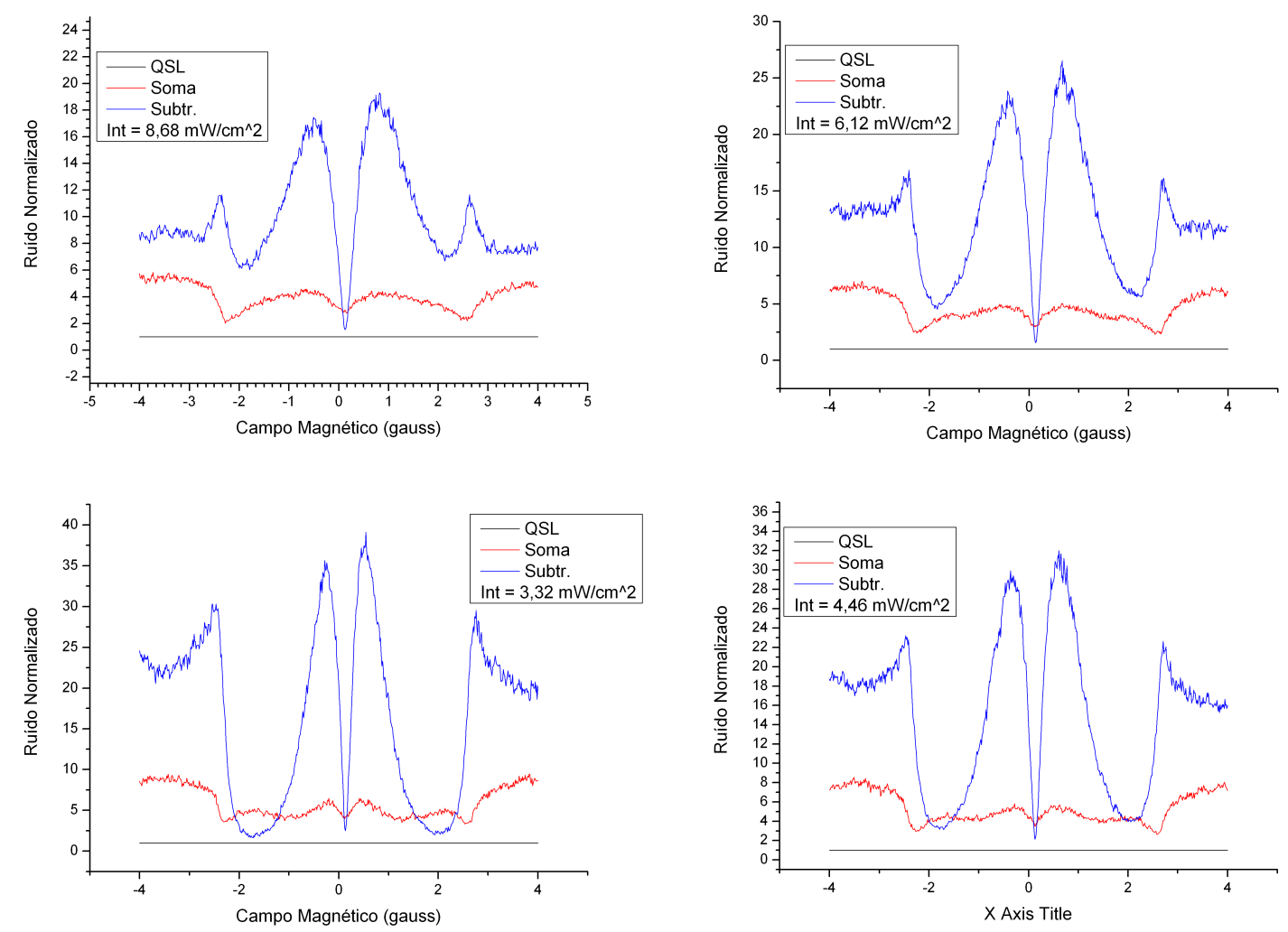

Figura 7.9: Espectros de Ruído de Soma de Subtração para Diferentes Intensidades

quer intensidade, sendo que para altas intensidades apenas em campo magnético próximo do zero há correlação e para qualquer outro valor de campos, as mesmas estão anticorrelacionadas. Para baixas intensidades, há mais duas regiões de correlação nos picos laterais, além do que ocorre para campo nulo. Este comportamento nos diz que a altas intensidades o espalhamento Raman contribui mais que a EIT para o ruído quando a diferença de frequência gerada pela campo magnético é igual à frequência de análise. Situação que muda para baixas intensidades, onde a EIT domina e gera correlações entre os campos nesta condição. 

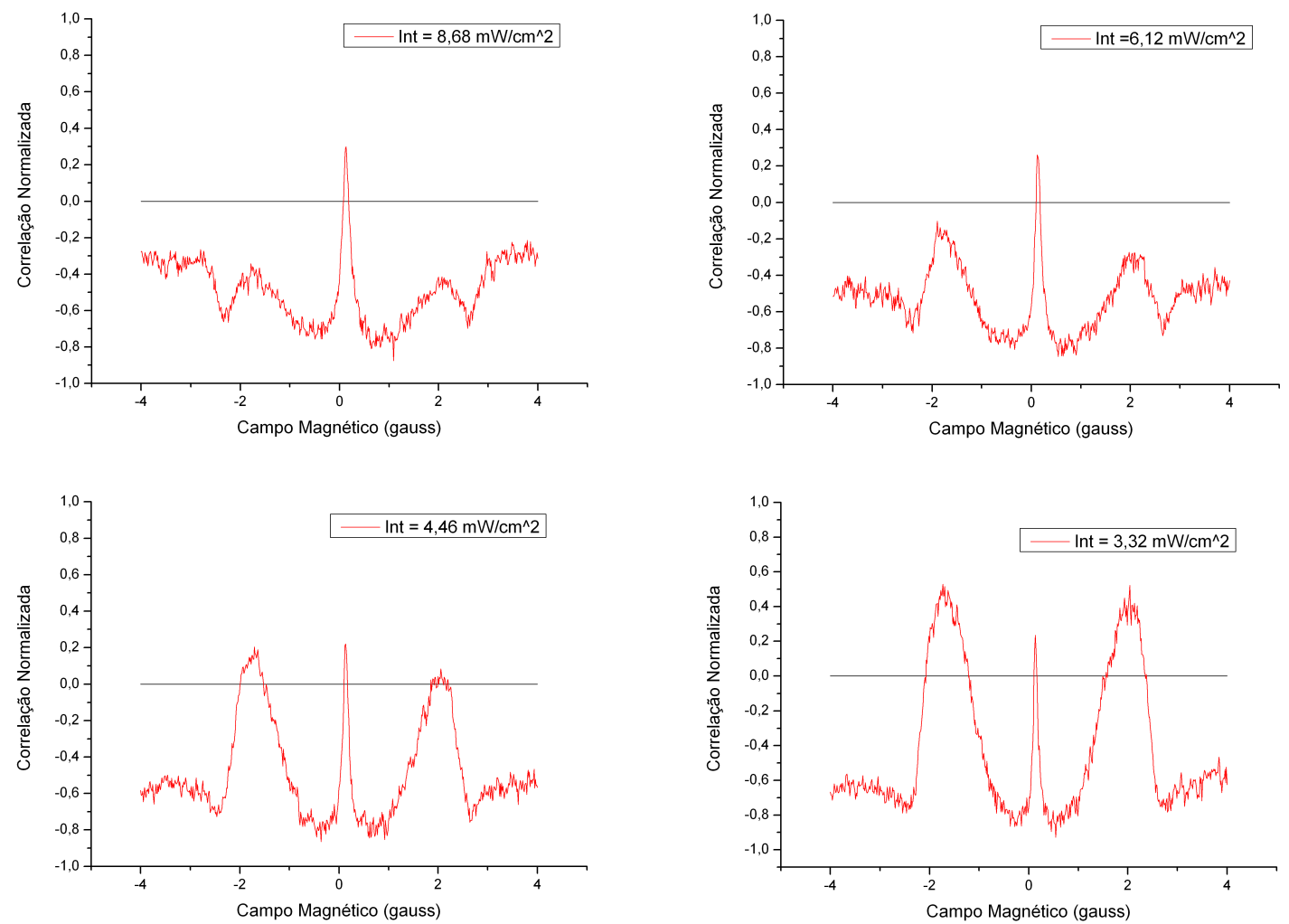

Figura 7.10: Correlações Normalizadas com a Separação feita por uma Lâmina de $\lambda / 4$. 

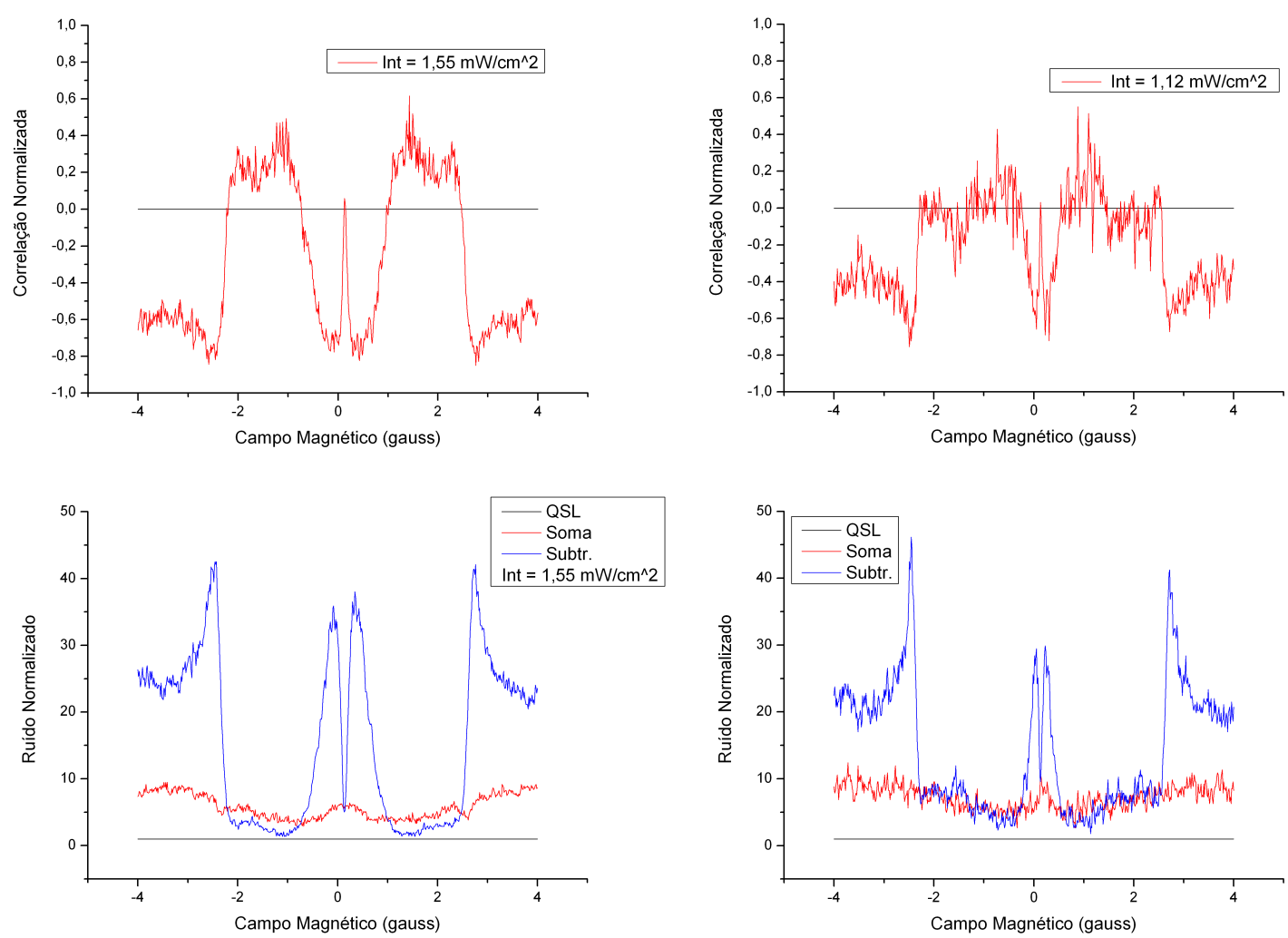

Figura 7.11: Espectro de ruído e correlações para baixas intensidades com a separação feita por uma lâmina de $\lambda / 4$. 


\section{Conclusões e Perspectivas}

Neste trabalho, foram analisados, experimental e teoricamente, as flutuações de um feixe laser após interagir com um sistema de dois níveis com degenerescências. Formulamos um modelo para as flutuações baseado na quatização do campo e tratando a amostra atômica como oticamente fina. Neste modelo, levamos em consideração a interação do átomo com os modos do campo não populados, que introduzem efeitos tanto no valor médio, taxa de decaimento da emissão espontânea, quanto nas flutuações, matriz de difusão das forças quânticas de Langevin. Calculamos as contribuições das autocorrelações de fase e amplitude do feixe incidente para as flutuações de amplitude do feixe transmitido após passagem pela célula de vapor. Estas autocorrelações, quando calculadas na ordem simétrica, dão as mesmas contribuições que o modelo semiclássico.

Fizemos medidas do espectro de ruído de soma e subtração para várias configurações de níveis na linha $D_{2}$ do ${ }^{85} R b$ usando um laser de diodo como campo de prova. Apresentamos nossas melhores medidas destes espectros para separação com lâminas de $\lambda / 2$ e $\lambda / 4$, assim como o coeficiente de correlação simetrizado e normalizado calculado a partir dos espectros de soma e subtração. Mostramos que há regiões de correlação e anticorrelação e que estas regiões mudam conforme variamos a intensidade. Uma possível explicação para este fenômeno é a competição entre a EIT e a transição Raman.

Observamos, como em medidas anteriores, a conversão de ruído de fase em ruído de amplitude pelos átomos. Também observamos que o ruído de soma muda sua forma para baixas intensidades, o que não é previsto pelo modelo considerando apenas as flutuações fase no feixe incidente. Como a forma do sinal se torna muito parecida com a previsão feita considerando as flutuações de intensidade do feixe incidente, acreditamos que estas passam a dominar em baixas intensidades.

Estes estudos visam a implementação de memórias quânticas, geração de estados nãoclássicos, emaranhamento entre ensembles de átomos, entre outros efeitos. A utilização de um laser de diodo, como já enfatizado anteriormente, se mostra inadequada para estas 
aplicações, por causa do seu grande ruído de fase. Para eliminar este problema, um laser de Titânio:Safira está sendo construído no laboratório. O desenvolvimento de um dispositivo como este já foi iniciado, mas devido a problemas com o laser de bombeio o trabalho avançou pouco e teve de ser interrompido.

Uma das perspectivas experimentais para a continuação do trabalho é utilizar o feixe coerente fornecido pelo Ti:Safira para obter compressão de ruído no regime de mistura de quatro ondas como em [66]. Outro grande problema que impede estas medidas é o ruído eletrônico a baixa potência, problema que está sendo contornado. Outra perspectiva é realizar estas mesmas medidas em uma armadilha magneto-ótica (MOT), que já está em funcionamento no laboratório.

Os objetivos com as duas abordagens são bem diferentes. Comparativamente, o trabalho com a célula de vapor é mais simples do ponto de vista experimental, pois o único grande cuidado a ser tomado é realizar uma blindagem magnética eficiente. Outra vantagem é que este meio é mais interessante para possíveis aplicações tecnológicas, devido à robustez e à facilidade de movimentação da célula em comparação com a MOT. Pelo fato dos átomos estarem praticamente parados na MOT, o alargamento Doppler é eliminado e as condições experimentais se tornam mais próximas da teoria desenvolvida neste trabalho. Neste tipo de sistema, esperamos observar aspectos físicos mais fundamentais. Devido à baixa velocidade dos átomos, entretanto, ele requer um alto grau de controle.

Outra perspectiva é a integração deste sistema com o outro experimento em curso no laboratório. A idéia é transferir o emaranhamento gerado pelo oscilador paramétrico ótico (OPO) para os átomos. Para isso, há um projeto de construção de um outro OPO que opera na frequência de interação com os átomos de rubídio. 


\section{APÊNDICE A - Hamiltoniano de Dipolo}

Considere um sistema físico formado por $N$ partículas carregadas, cada uma com carga $q_{k}$ e massa $m_{k}$. O hamiltoniano desse sistema será

$$
H_{0}=\sum_{k} \frac{1}{2 m_{k}} \mathbf{p}_{k}^{2}+U\left(\mathbf{r}_{1}, \mathbf{r}_{2}, \ldots, \mathbf{r}_{N}\right)
$$

onde $\mathbf{p}_{k}$ e $\mathbf{r}_{k}$ são o momento e a posição da partícula $k$, e $U$ é um potencial que contém a atração eletrostática entre as partículas.

Ao considerar a interação com um campo eletromagnético externo, definido pelos potenciais $\mathbf{A}(\mathbf{r}, t)$ e $\Phi(\mathbf{r}, t)$, e forte o suficiente para que o campo criado pelo movimento das partículas seja desprezível comparado ao campo externo, o hamiltoniano dos sistema partículas-campo será dado por [39]

$$
H=H_{f}+\sum_{k}\left[\frac{1}{2 m_{k}}\left(\mathbf{p}_{k}+\frac{q_{k}}{c} \mathbf{A}_{k}\right)^{2}+q_{k} \Phi_{k}+\mu_{k} \cdot \mathbf{B}_{k}\right]+U\left(\mathbf{r}_{1}, \mathbf{r}_{2}, \ldots, \mathbf{r}_{N}\right),
$$

onde $\mathbf{A}_{k}=\mathbf{A}\left(\mathbf{r}_{k}, t\right)$, o mesmo vale para $\mathbf{B}_{k}$ e $\Phi_{k}$, e $H_{f}$ é o hamiltoniano de campo livre, definido em (2.53). Aqui, foi usado o acoplamento mímino fazendo $\mathbf{p}_{k} \longrightarrow \mathbf{p}_{k}-\left(q_{k} / c\right) \mathbf{A}_{k}$ e foi adiconado o termo de interação com o spin. Em princípio, o campo eletromagnético é clássico, porém como estamos interessados em um modelo quântico, subtituiremos os campos pelos seus respectivos operadores definidos em (2.63). Desta forma, a expressão (A.2) fica

$$
\begin{aligned}
H & =H_{0}+H_{\gamma}+H_{1}+H_{2} \\
H_{1} & =\sum_{k}-\frac{q_{k}}{m_{k} c} \hat{\mathbf{p}}_{k} \cdot \hat{\mathbf{A}}_{k}+\hat{\mu}_{k} \cdot \hat{\mathbf{B}}_{k} \\
H_{2} & =\frac{q_{k}^{2}}{2 m_{k} c^{2}} \hat{\mathbf{A}}_{k}^{2}
\end{aligned}
$$

já considerando que $\nabla \cdot \hat{\mathbf{A}}\left(\mathbf{r}_{\mathbf{k}}, \mathbf{t}\right)=0$.

Com campo quantizado, o termo $H_{1}$ será responsável pelos processos de emissão e 
absorção, e $H_{2}$ será responsável pelo espalhamento. Este último termo, assim como a segunda parcela de $H_{1}$ estão relacionados a processos de energias muito mais altas do que as responsáveis pelos problemas tratados neste trabalho, por isso serão desconsiderados $[47,36,27]$. Levando em consideração $(2.8), \mathbf{p}_{k}=m_{k} \dot{\mathbf{r}}_{k}$, então

$$
\mathbf{p}_{k} \cdot \mathbf{A}_{k}=\frac{d}{d t}\left[\mathbf{r}_{k} \cdot \mathbf{A}_{k}\right]+m_{k} c \mathbf{r}_{k} \cdot \mathbf{E}_{k}
$$

Considerando que as partículas formam um átomo de um elétron e que a frequência do campo está no domínio ótico podemos usar a aproximação de longo comprimento de onda. Nestas condições, $\left|\mathbf{r}_{n}\right| \sim 0,1$ nm e $\lambda \sim 1 \mu m$, então $\left|\mathbf{k} \cdot \mathbf{r}_{n}\right| \ll 1$, então podemos substituir $\exp \left(i \mathbf{k} \cdot \mathbf{r}_{n}\right) \approx 1 \mathrm{em}(2.63)$, ou seja, $\mathbf{A}\left(\mathbf{r}_{k}, t\right) \approx \mathbf{A}(0, t)$.

Para o caso do campo clássico, essa aproximação equivale a um campo espacialmente constante interagindo com um dipolo formado pelo próton e pelo elétron, com hamiltoniano é dado por $H_{d}=e \mathbf{r} \cdot \mathbf{E}(t)$. Logo, era de se esperar que para um campo lentamente oscilante, independente de ser clássico ou quântico, obtivéssemos o mesmo hamiltoniano. Como pode ser visto, o segundo termo de ((A.6)) realmente dá origem ao hamiltoniano de dipolo, então, tudo que temos a fazer é, dentro da aproximação de longo comprimento de onda, anular o primeiro. Isso pode ser feito utilizando, na transformação de calibre (2.41), $\Lambda(\mathbf{r}, t)=-\mathbf{r} \cdot \mathbf{A}\left(\mathbf{r}_{k}, t\right)$, conhecida com transformação de Goppert-Mayer [37], portanto,

$$
\mathbf{A}^{\prime}\left(\mathbf{r}_{k}, t\right)=\mathbf{A}\left(\mathbf{r}_{k}, t\right)-\mathbf{A}(0, t)
$$

e $\mathbf{A}^{\prime}(0, t)=0$. Substituindo $\mathbf{A}\left(\mathbf{r}_{k}, t\right)$ por $\mathbf{A}^{\prime}\left(\mathbf{r}_{k}, t\right)$ em $((\mathrm{A} .6))$ e fazendo $\mathbf{r}_{k} \longrightarrow 0$ por meio da aproximação de longo comprimento de onda, eliminamos o primeiro termo e

$$
H_{1}=-\mathbf{d} \cdot \mathbf{E}(t)
$$

onde $\mathbf{d}=-e \mathbf{R}$ é o operador de dipólo, com $e$ sendo a carga fundamental e $\mathbf{R}$ o operador posição do elétron em relação ao próton. Esse hamiltoniano será utilizado em todos os modelos desenvolvidos nesse trabalho, independente de o campo ser ou não quantizado. Note que a condição $\nabla \cdot \mathbf{A}^{\prime}\left(\mathbf{r}_{\mathbf{k}}, \mathbf{t}\right)=0$ é satisfeita sempre que $\nabla \cdot \mathbf{A}\left(\mathbf{r}_{\mathbf{k}}, \mathbf{t}\right)=0$, ou seja, $\mathbf{A}^{\prime}\left(\mathbf{r}_{k}, t\right)$ também está definido no plano transverso à direção de propagação. 


\section{APÊNDICE B - Espaço de Liouville}

Em muitas aplicações da Mecânica Quântica em sistemas complexos, fica impraticável tratar o problema do operador densidade, mais específicamente a equação de Von Neumann, com a base definida no espaço de Hilbert. O operador densidade está definido no espaço dos operadores que atuam no espaço de Hilbert, chamado de espaço de Liouville [59]. Para deixar o problema mais tratável do ponto de vista teórico, usaremos a base na qual o operador densidade será expandido como pertencente ao espaço de Liouville. Expandidos nesta base, todo operador que atua no espaço de Hilbert se torna um vetor e os 'operadores' que atuam no espaço de Liouville são chamados de superoperadores.

Iniciaremos a formulação no espaço de Liouville considerando uma base discreta para o espaço de Hilbert, este é o caso tratado neste trabalho. Considere a equação de Von Neumann expandida numa base discreta de dimensão $N$,

$$
i \hbar \dot{\rho}_{i j}=\sum_{k}\left[H_{i k} \rho_{k j}-H_{k j} \rho_{i k}\right]
$$

Esta equação pode ser escrita da seguinte forma

$$
\begin{aligned}
i \hbar \dot{\rho}_{i j} & =\sum_{k l} £_{i j, k l} \rho_{k l}, \\
£_{i j, k l} & =H_{i k} \delta_{j l}-H_{j l}^{*} \delta_{i k},
\end{aligned}
$$

onde $£$ será sempre designado para o superoperador Liouvilliano, que realiza a evolução temporal no espaço de Liouville assim como o hamiltoniano faz no espaço de Hilbert. Esta última equação pode ser vista como uma equação tipo Schrödinger em um espaço de dimensão $N^{2}$. Tendo em vista a equação (B.1), definimos a base no espaço de Liouville como $\| i j\rangle \equiv|i\rangle\langle j|$, tal que qualquer operador $\hat{O}$ seja expandido como

$$
\left.\hat{O} \longrightarrow \| O\rangle=\sum_{i j} O_{i j} \| i j\right\rangle
$$

O conjugado hermitiano de $\| i j\rangle$ é definido de forma semelhante, $\langle i j \| \equiv \mid j\rangle\langle i|=(\| i j\rangle)^{\dagger}$, de modo que vale a seguinte relação, $\langle i j\|=\| j i\rangle$. Desta forma, podemos escrever o 
conjugado hermitiano de $\hat{O}$ como

$$
\hat{O}^{\dagger}=\sum_{i j} O_{i j}^{*}|j\rangle\langle i| \equiv\langle O \|
$$

Ao olhar para (B.2), vemos que $O_{i j}=\langle i|O| j\rangle=\operatorname{Tr}[|j\rangle\langle i| \hat{O}]$. Com esta motivação, definimos o produto escalar entre a base e o operador $\hat{O}$ como

$$
\langle i j \| O\rangle \equiv \operatorname{Tr}\left[(|i\rangle\langle j|)^{\dagger} \hat{O}\right]
$$

tal que $\left.\| O\rangle=\sum_{i j} \| i j\right\rangle\langle i j \| O\rangle$. Portanto, valem as relações de ortogonalidade e fechamento

$$
\begin{aligned}
\left.\sum_{i j} \| i j\right\rangle\langle i j \| & =1, \\
\langle i j \| k l\rangle & =\delta_{i k} \delta_{j l},
\end{aligned}
$$

de modo que qualquer superoperador pode ser expandido como

$$
\begin{aligned}
S & \left.=\sum_{i j, k l} S_{i j, k l} \| i j\right\rangle\langle k l \|, \\
S_{i j, k l} & =\langle i j\|S\| k l\rangle .
\end{aligned}
$$

Usando a relação (B.4) podemos mostrar que para qualquer operadores $\hat{A}$ e $\hat{B}$, o produto interno no espaço de Liouville fica

$$
\langle A \| B\rangle \equiv \operatorname{Tr}\left[\hat{A}^{\dagger} \hat{B}\right]
$$

A média de um superoperador é definida como uma extensão de (B.8)

$$
\langle A\|S\| B\rangle \equiv \operatorname{Tr}\left[\hat{A}^{\dagger} S \hat{B}\right]
$$

onde, $S \hat{B}$ deve ser uma função de $\hat{B}$ ou $\hat{A} S$ deve ser uma função de $\hat{A}$ para que a operação de traço faça sentido.

Com excessão das três primeira equações, todos resultados mostrados até agora são gerais para o espaço de Liouville e valem para qualquer operador. Daqui em diante focaremos no operador densidade iniciando pela equação de movimento. Escrevemos a equação, similar à de Schrödinger,

$$
\left.\left.i \hbar \frac{d}{d t} \| \rho(t)\right\rangle=£(t) \| \rho(t)\right\rangle
$$

No caso do operador densidade, $£(t) \hat{\rho}(t) \equiv[H(t), \hat{\rho}(t)]$ e, ao introduzir a relação de 
fechamento na equação de movimento, recuperamos (B.1). Portanto, ela é equivalente à equação de Von Neumann. Esta é uma das grandes vantagens de se trabalhar no espaço de Liouville, nele a equação de Von Neumann possui a mesma forma que a de Schrödinger no espaço de Hilbert.

Se a função mencionada do parágrafo anterior for $£ \hat{\rho}=\hat{O} \hat{\rho}$, onde $\hat{O}$ é um operador qualquer, então

$$
\begin{array}{r}
(\hat{O} \hat{\rho})_{i j} \longrightarrow \mathcal{L}(\hat{O})_{i j, k l} \hat{\rho}_{k l} \\
\mathcal{L}(\hat{O})_{i j, k l}=O_{i k} \delta_{j l}
\end{array}
$$

onde $\mathcal{L}(\hat{O})_{i j, k l}$ representa o efeito de $\hat{O}$ atuando em $\hat{\rho}$ pela esquerda e a seta significa a equivalência ao passar para o espaço de Liouville. A contribuição de $£ \hat{\rho}=\hat{\rho} \hat{O}$ será,

$$
\begin{array}{r}
(\hat{\rho} \hat{O})_{i j} \longrightarrow \mathcal{R}(\hat{O})_{i j, k l} \hat{\rho}_{k l} \\
\mathcal{R}(\hat{O})_{i j, k l}=O_{l j} \delta_{i k}
\end{array}
$$

com $\mathcal{R}(\hat{O})_{i j, k l}$ representando o efeito de $\hat{O}$ atuando em $\hat{\rho}$ pela direita.

Para mais de um operador atuando em $\rho$ pela esquerda, os superoperadores no espaço de Liouville apareceram na mesma ordem em que os operadores no espaço de Hilbert. Para operadores que atuam à direta, a substituição deve ser feita tal que a ordem de proximidade de $\rho$ seja mantida, ou seja, o superoperador que atuará primeiro em $\| \rho\rangle$ será o referente ao operador que estiver mais próximo de $\rho$. Para o caso em que $\rho$ está entre dois operadores, como em (5.33), a ordem não importa, pois, a partir das definições (B.11)-(B.10), pode-se mostrar que

$$
\left[\mathcal{L}(\hat{O}), \mathcal{R}\left(\hat{O}^{\prime}\right)\right]=0
$$

Para um operador formado pela soma de outros, também segue diretamente de (B.11)(B.10) que

$$
\mathcal{L}\left(\hat{O}_{1}+\hat{O}_{2}\right)_{i j, k l}=\mathcal{L}\left(\hat{O}_{1}\right)_{i j, k l}+L\left(\hat{O}_{2}\right)_{i j, k l}
$$

e o mesmo vale para $\mathcal{R}(\hat{O})_{i j, k l}$. Essa propriedade releva a aditividade ao se passar para o espaço de Liouville.

Note que a passagem para o espaço de Liouville é somente um rearrajo das componentes do operador densidade na forma de um vetor, o que também foi feito nos capítulos 3 e 4. Deste modo, ordenação da base no espaço de Hilbert, há várias possibilidades de ordenação para a base no espaço de Liouville. Esta ordenação é arbitária, mas deve ser es- 
colhida no início do cálculo e mantida até o final. De fato, todo o formalismo desenvolvido neste apêndice foi motivado para poder se trabalhar com graus de liberdade maiores sem ter que escrever as matrizes explicitamente, o que seria impraticável para muitos casos. 


\section{APÊNDICE C - Espectro de Ruido e Teorema de Wiener-Khinthchine}

Neste apêndice faremos uma revisão do espectro de ruído. Considere um operador dependente do tempo, $\hat{o}(t)$, a sua expressão no domínio da frequência será

$$
\hat{o}(\omega)=\frac{1}{\sqrt{2 \pi}} \int_{-\infty}^{\infty} d t e^{-i \omega t} \hat{o}(t)
$$

Se tomamos $\langle\hat{o}(\omega)\rangle$, esta será a transformada de Fourrier de $\langle\hat{o}(t)\rangle$. A transformada é necessária porque os aparatos que utilizamos para realizar as medidas, como o analisador de espectro, as fazem no domínio da frequência. Mas há um problema com esta média, pois, caso o operador no domínio do tempo possua um valor estacionário, esta integral irá divergir para tempos longos. Sendo assim, precisamos de uma função que seja convergente para tempos longos e possa ser medida no domínio da frequência. Pelo fato de as funções de correlação no domínio do tempo se anularem para grandes diferenças de tempo, definiremos uma função na qual a média em (C.1) é substituida pela correlação.

A correlação entre os operadores $\hat{o}$ e $\hat{o}^{\prime}$ no domínio da frequência fica

$$
\left\langle\hat{o}(\omega) \hat{o}^{\prime}\left(\omega^{\prime}\right)\right\rangle=\frac{1}{2 \pi} \int_{-\infty}^{\infty} \int_{-\infty}^{\infty} d t^{\prime} d t e^{-i \omega t} e^{-i \omega^{\prime} t^{\prime}}\left\langle\hat{o}(t) \hat{o}^{\prime}\left(t^{\prime}\right)\right\rangle .
$$

Fazemos a mudança de variáveçl $t^{\prime} \rightarrow t+\tau$, então

$$
\left\langle\hat{o}(\omega) \hat{o}^{\prime}\left(\omega^{\prime}\right)\right\rangle=\frac{1}{2 \pi} \int_{-\infty}^{\infty} \int_{-\infty}^{\infty} d t d \tau e^{-i\left(\omega+\omega^{\prime}\right) t} e^{-i \omega^{\prime} \tau} C_{o, o^{\prime}}(t, t+\tau),
$$

onde $C_{o, o^{\prime}}(t, t+\tau)=\left\langle\hat{o}(t) \hat{o}^{\prime}(t+\tau)\right\rangle$ é a função de correlação temporal. Se impomos a condição que a função de correlação é invariante por translação temporal, ela dependerá somente da diferença de tempo, ou seja, $C_{o, o^{\prime}}(t, t+\tau)=C_{o, o^{\prime}}(t, t+\tau) \equiv C_{o, o^{\prime}}(\tau)$. Então,

$$
\begin{aligned}
\left\langle\hat{o}(\omega) \hat{o}^{\prime}\left(\omega^{\prime}\right)\right\rangle & =\delta\left(\omega+\omega^{\prime}\right) S_{o, o^{\prime}}^{p}\left(\omega^{\prime}\right) \\
S_{o, o^{\prime}}^{p}\left(\omega^{\prime}\right) & =\int_{-\infty}^{\infty} d \tau e^{-i \omega^{\prime} \tau} C_{o, o^{\prime}}(\tau)
\end{aligned}
$$


onde $S_{o, o^{\prime}}^{p}\left(\omega^{\prime}\right)$ é o espectro de potência de $\hat{o}$ e $\hat{o}^{\prime}$ na frequência $\omega^{\prime}$. O espectro de potência era justamento o que estávamos procurando, pois forma um par transformada de Fourrier com algo que converge para $\tau$ grande e, que no caso do ruído branco é uma delta de Dirac definida na origem [52]. A expressão (C.3) é conhecida como teorema de WienerKhinthchine.

Os operadores $\hat{o}$ e $\hat{o}^{\prime}$ podem ser escritos como

$$
\begin{aligned}
\hat{o}(t) & =\langle\hat{o}\rangle+\delta \hat{o}(t), \\
\hat{o}^{\prime}(t) & =\left\langle\hat{o}^{\prime}\right\rangle+\delta \hat{o}^{\prime}(t),
\end{aligned}
$$

onde $\langle\hat{o}\rangle$ é o valor médio estacionário e $\delta \hat{o}(t)$ é uma flutuação dependente do tempo com média nula. Desta forma, a função de correlação se torna

$$
C_{o, o^{\prime}}(\tau)=\langle\hat{o}\rangle\left\langle\hat{o}^{\prime}\right\rangle+C V_{o, o^{\prime}}(\tau)
$$

onde $C v_{o, o^{\prime}}(\tau)=\left\langle\delta \hat{o}(t) \delta \hat{o}^{\prime}(t+\tau)\right\rangle$ é a função de covariância. Desta forma, o espectro de potência pode ser escrito como

$$
\begin{aligned}
& S_{o, o^{\prime}}^{p}\left(\omega^{\prime}\right)=\langle\hat{o}\rangle\left\langle\hat{o}^{\prime}\right\rangle \delta\left(\omega^{\prime}\right)+S_{o, o^{\prime}}\left(\omega^{\prime}\right), \\
& S_{o, o^{\prime}}\left(\omega^{\prime}\right)=\int_{-\infty}^{\infty} d \tau e^{-i \omega^{\prime} \tau} C V_{o, o^{\prime}}(\tau)
\end{aligned}
$$

onde $S_{o, o^{\prime}}\left(\omega^{\prime}\right)$ é o espectro de ruído e pode ser calculado diretamente no domínio da frequência como

$$
\left\langle\delta \hat{o}(\omega) \delta \hat{o}^{\prime}\left(\omega^{\prime}\right)\right\rangle=\delta\left(\omega+\omega^{\prime}\right) S_{o, o^{\prime}}\left(\omega^{\prime}\right)
$$




\section{Referências Bibliográficas}

[1] M. Fleishhauer; A. Imamoglu; J. P. Marangos. Electromagnetically induced transparency: Optics in coherent media. Review of Modern Physics, 77:633-673, 2005.

[2] E. Arimondo. Coherent population trapping in laser spectroscopy. Progress in Optics, 35:257-354, 1996.

[3] G. Alzetta; A. Gozzini; L. Moi; G. Orriols. Coherent effects on the zeeman sublevels of hyperfine states in optical pumping of $\mathrm{rb}$ by monomode diode laser. Il Nuovo Cimento, 36B(5), May 1976.

[4] K.-J. Kolle; A. Imamoglu; S. E. Harris. Observation of electromagnetically induced transparency. Phys. Rev. Lett., 66(20), May 1991.

[5] M. Fleischhauer; M. D. Lukin. Dark-state polaritons in electromagnetically induced transparency. Phys. Rev. Lett., 84(22):5094-5097, May 2000.

[6] M. A. Nielsen; I. L. Chuang. Quantum Computation and Quantum Information. Cambridge Univ. Press, 2000.

[7] J. Cviklinski; J. Ortalo; J. Laurat; A. Bramati; M. Pinard; E. Giacobino. Reversible quantum interface for tunable single-sideband modulation. Phys. Rev. Lett., 101 (133601), Sep 2008.

[8] Jürgen Appel; Eden Figueroa; Dmitry Korystov; M. Lobino; A. I. Lvovsky. Quantum memory for squeezed light. Phys. Rev. Lett., 100(093602), Mar 2008.

[9] Kazuhito Honda; Daisuke Akamatsu; Manabu Arikawa; Yoshihiko Yokoi; Keiichirou Akiba; Satoshi Nagatsuka; Takahito Tanimura; Akira Furusawa; Mikio Kozuma. Storage and retrieval of a squeezed vacuum. Phys. Rev. Lett., 100(093601), Mar 2008.

[10] M.D. Eisaman; A. André; F. Massou; M. Fleischhauer; A.S. Zibrov; M.D. Lukin. Electromagnetically induced transparency with tunable single-photon pulses. Nature, 438(04315), Dec 2005.

[11] D. N. Matsukevich; T. Chanelie're; S. D. Jenkins; S.-Y. Lan; T. A. B. Kennedy; A. Kuzmich. Entanglement of remote atomic qubits. Phys. Rev. Lett., 96(030405), Jan 2006.

[12] T. Chanelière; D. N. Matsukevich; S. D. Jenkins; S.-Y. Lan; T. A. B. Kennedy; A. Kuzmich. Storage and retrieval of single photons transmittedbetween remote quantum memories. Nature, 438(04327), Dec 2005. 
[13] M. Fleischhauer; T. Richter. Pulse matching and correlations of phase fluctuations in $\lambda$ systems. Phys. Rev. A, 51(3):2430-2442, March 1995.

[14] C. L. Garrido Alzar; M. França Santos; P. Nussenzveig. Entanglement between two macroscopic fields by coherent atom-mediated exchange of photons. 2003.

[15] P. Barberis-Blostein; N. Zagury. Field correlations in electromagnetically induced transparency. Phys. Rev. A, 70(053827), Nov 2008.

[16] P. Barberis-Blostein. Two-photon detuning and decoherence in cavity electromagnetically induced transparency for quantized fields. Phys. Rev. A, 77(013821), Jan 2008.

[17] A. Sinatra. Quantum correlations of two optical fields close to electromagnetically induced transparency. 2007.

[18] C. L. Garrido Alzar; L. S. Cruz; J. G. Aguirre-Gómez; M. França Santos; P. Nussenzveig. Super-poissonian photon statistics and correlations between pump and probe fields in electromagnetically induced transparency. Europh. Lett., 61(4):485, 2003.

[19] L.S. Cruz; D. Felinto; J. G. Aguirre-Gómez; M. Martinelli1; P. Valente; A. Lezama; and P. Nussenzveig. Laser-noise-induced correlations and anti-correlations in electromagnetically induced transparency. Eur. Phys. J. D, 41(3):531-539, Mar 2007.

[20] M. Martinelli; P. Valente; H. Failache; D. Felinto; L. S. Cruz; P. Nussenzveig; A. Lezama. Noise spectroscopy of nonlinear magneto-optical resonances in rb vapor. Phys. Rev. A, 69:043809, 2004.

[21] A. Lezama; P. Valente; H. Failache; M. Martinelli; P. Nussenzveig. Numerical investigation of the quantum fluctuations of optical fields transmitted through an atomic medium. Phys. Rev. A, 77(013806), 2008.

[22] C. L. Garrido Alzar. Estudo de Propriedades Quânticas dos Feixes Sonda e de Bombeio na Transparência Induzida por Laser. PhD thesis, Universidade de São Paulo, 2002.

[23] J. G. Aguirre-Gómez. Correlações e Ruído de Intensidade e de Fase em Transparência Induzida Eletromagneticamente: Experimentos e Teorias. PhD thesis, Universidade de São Paulo, 2004.

[24] P. H. Valente. Estudio de Efectos Transitorios Asociados a Resonancias Coherentes. PhD thesis, Universidad de La Republica - Uruguai, 2004.

[25] L. S. da Cruz. Flutuações de Campos Eletromagnéticos Interagindo com Meios Atômicos Coerentemente Preparados. PhD thesis, Universidade de São Paulo, 2005.

[26] C. Cohen-Tannoudji; B.Diu; F. Laloe. Quantum Mechanics. Wiley-VCH,Vol-1-2, 2005.

[27] K. Gottifried; T.-M. Yan. Quantum Mechanics: Fundamentals. Spring-Verlag,2 ed, 2003.

[28] A. F. R. de Toledo Piza. Mecânica Quântica. EDUSP, 2005. 
[29] J. J. Sakurai. Modern Quantum Mechanics. Addison-Wesley, 1994.

[30] C. W. Gardiner. Quantum Noise. Springer-Verlag, 1991.

[31] L. I. Schiff. Quantum Mechanics. McGraw-Hill Book Company, 1949.

[32] D. Stoler. Equivalence classes of minimum uncertainty packets. Phys. Rev. D, 1(12): 3217-3219, Jun 1970.

[33] H. Goldstein. Classical Mechanics. Addiason-Wesley Publishing Company, 2ed, 1980.

[34] M. Weissbluth. Atoms and Molecules. Academic Press-New York, 1978.

[35] R. Loudon. The Quantum Theory of Light. Oxford Science Publications, 3ed, 2000.

[36] J. J. Sakurai. Advanced Quantum Mechanics. Addison-Wesley, 1967.

[37] C. Cohen-Tannoudji; J. Dupont-Roc; G. Grynberg. Photons and Atoms-Introduction to Quantum Electrodynamics. Wiley, 1992.

[38] W. Vogel; D.-G. Welsch. Quantum Optics. Wiley, 2001.

[39] J. D. Jackson. Classical Electrodynamics. Wiley, 1962.

[40] M. S. Scully, M. O.; Zubairy. Quantum Optics. Cambridge University Press, 1997.

[41] D. F. Walls; G. J. Milburn. Quantum Optics. Spring-Verlag, 1994.

[42] L.Mandel; E. Wolf. Optical Coherence and Quantum Optics. Cambridge University Press, 1995.

[43] Roy J. Glauber. The quantum theory of optical coherence. Phys. Rev., 130(6): 2529-2539, Jun 1963.

[44] R. J. Glauber. Coherent and incoherent states of the radiation field. Phys. Rev., 131 (6):2766-2788, Sep 1963.

[45] D. T. Pegg; S. M. Barnett. On the hermitian optical phase operator. J. Mod. Opt., 36(1):7-19, 1989.

[46] L. Allen; J. H. Eberly. Optical Ressonance and two-level Atoms. Dover, 1974.

[47] C. Cohen-Tannoudji; J. Dupont-Roc; G. Grynberg. Atom-Photon Interactions-Basic Processes and Applications. Wiley, 1992.

[48] H. Carmichael. An Open Systems Approach to Quantum Optics. Springer, 1993.

[49] B. P. Blostein. Correlações de Campo na Transparência Induzida Eletromagneticamente. PhD thesis, Universidade Federal do Rio de Janeiro, 2004.

[50] R. R. Puri. Mathematical Methods of Quantum Optics. Spring-Verlag, 2001.

[51] J. Weiner; P.-T. Ho. Light-Matter Interactions: Fundamentals and Applications. Wiley, 2003. 
[52] C. W. Gardiner. Handbook of Stochastic Methods. Springer-Verlag, 1991.

[53] van Kampen N. G. Stochastic Procosses in Physics and Chemistry. Elsevier, sei lah.

[54] T. Tomé; M. J. de Oliveira. Dinâmica Estocástica e Irreversibilidade. EDUSP, 2001.

[55] A. S. Villar. Emaranhamento Multicolor entre Feixes Intensos de Luz. PhD thesis, Universidade de São Paulo, 2007.

[56] A. Villar. The conversion of phase to amplitude fluctuations of a light beam by an optical cavity. Am. J. Phys., 2008.

[57] J. Dalibard; C. Cohen-Tannoudji. Laser cooling below the doppler limit by polarization gradients: simple theoretical models. J. Opt. Soc. Am. B, 6(11):2023-2045, Nov 1989.

[58] U. Fano. Pressure broadening as a prototype of relaxation. Phys. Rev, 131(1):259258, May 1963.

[59] S. Mukamel. Principles of Nonlinear Optical Spectroscopy. Orford Press, 1995.

[60] M. Martinelli. Compressão de Ruído Quântico e Efeitos Transeversos em Osciladores Paramétricos Óticos. PhD thesis, Universidade de São Paulo, 2002.

[61] L. Ricci; M. Weidemuller; T. Esslinger; A. Hemmerich; C. Zimmermann; V. Vuletic; W. Konig;T. W. Hansch. Electromagnetically induced transparency: Optics in coherent media. Opt. Commun, 177:541-549, 1995.

[62] T. Yabuzaki; T. Mitsui; U. Tanaka. New type of high-resolution spectroscopy with a diode laser. Phys. Rev. Lett., 67(18):2453-2456, Oct 1970.

[63] R. Walser; P. Zoller. Laser-noise-induced polarization fluctuations as a spectroscopic tool. Phys. Rev. A, 49:5067, 1994.

[64] A. Yariv. Quantum Electronics. Wiley, 1988.

[65] H.-A. Bachor; T. C. Ralph. A Guide to Experiments in Quantum Optics. Willey ,2 ed, 2004.

[66] C. F. McCormick; V. Boyer; E. Arimondo; P. D. Lett. Strong relative intensity squeezing by 4 -wave mixing in rb vapor. Quantum Electronics and Laser Science Conference QELS '0\%, (6-11), May 2007. 